\title{
A THREE DIMENSIONAL VORTEX PARTICLE-PANEL CODE FOR MODELING PROPELLER-AIRFRAME INTERACTION
}

\author{
A Thesis \\ presented to \\ the Faculty of California Polytechnic State University, \\ San Luis Obispo
}

\author{
In Partial Fulfillment \\ of the Requirements for the Degree \\ Master of Science in Aerospace Engineering
}

by

Jacob Calabretta

June 2010 
(c) 2010

Jacob Calabretta

ALL RIGHTS RESERVED 


\section{COMMITTEE MEMBERSHIP}

TITLE :

A THREE DIMENSIONAL VORTEX PARTICLE-PANEL CODE

FOR MODELING PROPELLER-AIRFRAME INTERACTION

AUTHOR: Jacob Calabretta

DATE SUBMITTED: June 2010

Dr. Rob A. McDonald

Advisor and Committee Chair

Aerospace Engineering

Dr. David D. Marshall

Committee Member

Aerospace Engineering

Dr. Larry L. Erickson

Committee Member

NASA-Ames Research Center

Dr. Timothy Takahashi

Committee Member

Northrop Grumman Corp. 


\begin{abstract}
A Three Dimensional Vortex Particle-Panel Code for Modeling Propeller-Airframe Interaction

Jacob Calabretta
\end{abstract}

Analysis of the aerodynamic effects of a propeller flowfield on bodies downstream of the propeller is a complex task. These interaction effects can have serious repercussions for many aspects of the vehicle, including drag changes resulting in larger power requirements, stability changes resulting in adjustments to stabilizer sizing, and lift changes requiring wing planform adjustments.

Historically it has been difficult to accurately account for these effects at any stage during the design process. More recently methods using Euler solvers have been developed that capture interference effects well, although they don't provide an ideal tool for early stages of aircraft design, due to computational cost and the time and expense of setting up complex volume grids. This research proposes a method to fill the void of an interference model useful to the aircraft conceptual and preliminary designer.

The proposed method combines a flexible and adaptable tool already familiar to the conceptual designer in the aerodynamic panel code, with a pseudo-steady slipstream model wherein rotational effects are discretized onto vortex particle point elements. The method maintains a freedom from volume grids that are so often necessary in the existing interference models. In addition to the lack of a volume grid, the relative computational simplicity allows the aircraft designer the freedom to rapidly test radically different configurations, including more unconventional designs like the channel wing, thereby providing a much broader design space than otherwise possible.

Throughout the course of the research, verification and validation studies were conducted to ensure the most accurate model possible was being applied. Once the vortex particle scheme had been verified, and the ability to model an actuator disk with vortex particles had been validated, the overall product was compared against propeller-wing wind tunnel results conducted specifically as benchmarks for numerical methods.

The method discussed in this work provides a glimpse into the possibility of pseudo-steady interference modeling using vortex particles. A great groundwork has been laid that already provides reasonable results, and many areas of interest have been discovered where future work could improve the method further. The current state of the method is demonstrated through simulations of several configurations including a wing and nacelle and a channel wing. 


\section{ACKNOWLEDGMENTS}

My tenure at California Polytechnic State University, San Luis Obispo has been a formative experience, and I owe a great deal of my success to the family, friends, classmates, and teachers who have supported me along the way. Without the unique help and inspiration provided to me from each of these sources it is difficult to imagine being where I am today.

My advisor, Dr. McDonald, has been instrumental in my development as an engineer. The constant passion and depth of knowledge that Dr. McDonald possesses helped to fuel my own desire to understand and contribute to the current state of aerospace engineering knowledge through participation in the Aerospace Sciences Meeting. I must also thank him for allowing me to participate on the sponsored thesis project, the funding of which helped pay for graduate school tuition. In addition I would like to thank Craig Nickol, the technical monitor for NASA Research Announcement Grant NNX07AO14A, which supported this work.

I owe gratitude to the many friends I have made while here at Cal Poly. There is not space to list them all, but each and every one helped in their own way. My roommates through the years have always been there to provide a needed distraction from often overwhelming amounts of work. My AERO friends have been my brothers (and sisters) in arms, standing together against the seemingly insurmountable tasks assigned to us and rising each time to meet the challenge. I have no doubt that we will remain in contact. Of course my friends of different majors contributed just as much, helping to maintain my sanity and allow me to escape work for much needed breaks. Thank you for your help in maintaining balance in my life.

Lastly, I would like to thank my family. I'm lucky to have such an amazing group of people who support me so unwaveringly. You've congratulated me on my accomplishments, but equally importantly, you've been there during all the lows to put me back together again, and your love and sacrifices are deeply appreciated. Mom and Pop, without writing another document this long I can't begin to describe everything that you have done for me. The examples you've set and lessons you've taught have enabled me to reach this point, and I know that you've provided me everything I need to start a family as wonderful as the one you've created. Phil, Nikki, and Gabrielle, I know you all have wonderful things in your future, and I can't wait to see what you will accomplish. Nonna, Grandma, and Grandpa, I'm only beginning to understand how lucky I have been to have you so close by all these years. Your love and company has been a gift I will always treasure. Thank you all. 


\section{Table of Contents}

Abstract iv

Acknowledgments $\quad$ v

Table of Contents ix

List of Tables $\quad x$

List of Figures xvi

Nomenclature xvii

1 Introduction 1

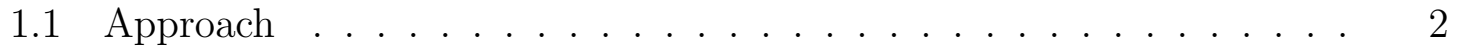

1.1.1 Crocco's Theorem . . . . . . . . . . . . . . . . . . 3

1.2 Document Roadmap . . . . . . . . . . . . . . . . . . . 3

$\begin{array}{lll}2 & \text { Comparison with Historical Methods } & 6\end{array}$

2.1 Current Applications of Vortex Particles . . . . . . . . . . . . 7

2.1 .1 Structural Aerodynamics Using Vortex Particles . . . . . . . . 7

2.1 .2 Vortex Particle Aircraft Wakes $\ldots \ldots \ldots \ldots$

2.1 .3 Helicopter Rotor Wakes . . . . . . . . . . . . . . . . . . . . 10

2.2 Propeller-Airframe Interaction History $\ldots \ldots \ldots \ldots \ldots$

2.2 .1 Actuator Disk . . . . . . . . . . . . . . . . . . . . . . 12

2.2 .2 Lifting Line Model . . . . . . . . . . . . . . . . . . . . . 16

2.2 .3 Rotating CFD grid . . . . . . . . . . . . . . . . . . . . 18

2.2 .4 Paneled Propeller Method . . . . . . . . . . . . . . . . . . . . 19

$2.2 .5 \quad$ Frequency Domain Panel Method . . . . . . . . . . . . 20

2.2 .6 Summary of Key Differences of Present Research . . . . . . . 21

\begin{tabular}{|lll}
3 & Vortex Particle Theory & 23
\end{tabular}

3.1 Fundamentals . . . . . . . . . . . . . . . . . . . . . . . . . . 23

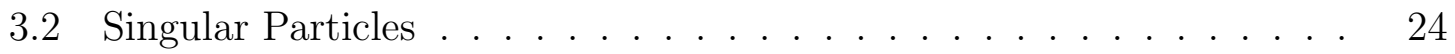


3.3 Regularized Particles . . . . . . . . . . . . . . . . . . . . . . . . . . . 27

3.4 Viscous Diffusion . . . . . . . . . . . . . . . . . . . . . . . . . . . 30

3.5 Evolution Equations $\ldots \ldots \ldots \ldots$

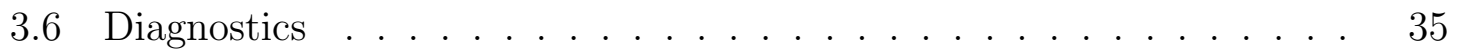

3.6 .1 Linear Diagnostics $\ldots \ldots \ldots \ldots$

3.6 .2 Quadratic Diagnostics $\ldots \ldots \ldots \ldots$

4 Particle Scheme Verification $\quad 40$

4.1 Particle Discretization . . . . . . . . . . . . . . . . . . . . . . 40

4.1 .1 Initial Strength Assignment . . . . . . . . . . . . . . . . 43

4.2 Beale's Method . . . . . . . . . . . . . . . . . . . . . . . . . 45

4.3 Vortex Core Size Optimization . . . . . . . . . . . . . . . . . . . . 47

4.4 Third Order Low Storage Runge-Kutta Solver . . . . . . . . . . . . . 49

4.4 .1 Round-off Error Removal . . . . . . . . . . . . . . . . . . . . . 49

4.4 .2 Low Storage . . . . . . . . . . . . . . . . . . . . . . . 52

4.5 Initial Particle Parameters . . . . . . . . . . . . . . . . . . . 53

4.6 Time Evolution . . . . . . . . . . . . . . . . . . . . . . . . . . . . . . 55

4.6 .1 Results . . . . . . . . . . . . . . . . . . . . . . . 56

$\begin{array}{lll}5 & \text { Analytical Actuator Disk Theory } & 61\end{array}$

5.1 Lightly Loaded Actuator Disk . . . . . . . . . . . . . . . . . . . 62

5.1 .1 Theory $\ldots \ldots \ldots \ldots \ldots$

5.1 .2 Actuator Disk with Elliptic Loading. . . . . . . . . . . . . . 67

5.1 .3 Actuator Disk with Parabolic Loading _... . . . . . . . . 69

5.2 Heavily Loaded Actuator Disk . . . . . . . . . . . . . . . . . . . . 71

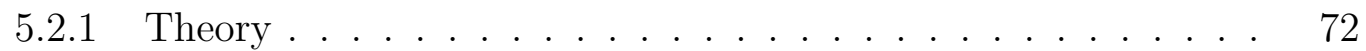

5.2 .2 Actuator Disk with Parabolic Loading _... . . . . . . . 74

5.3 The Actuator Disk Total Pressure Jump . . . . . . . . . . . . 76

5.3 .1 Effect of Efficiencies on Pressure Jump . . . . . . . . . . . . 81

5.3 .2 Method of Implementation $\ldots \ldots \ldots \ldots$

6 Particle Actuator Disk Validation 86

6.1 Particle Discretization . . . . . . . . . . . . . . . . . . 86

6.1 .1 Vortex Cores Sizing . . . . . . . . . . . . . . . . . 87

6.1 .2 Particle Strength Assignment _. . . . . . . . . . . . . . 88

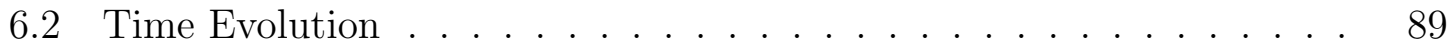

6.3 Simulation Results $\ldots \ldots \ldots \ldots$

$6.3 .1 \quad$ Matching $\hat{a}$

6.3 .2 Matching Axial Velocity at the Disk . . . . . . . . . . . 98

6.3 .3 Matching Vorticity Distribution Slope. . . . . . . . . . . . . 102

6.3 .4 Matching Maximum Vorticity . . . . . . . . . . . . . . . 106

6.3 .5 Conclusions from Different Matching Schemes . . . . . . . . 110 
6.3 .6 Smoothed Streamtube Vorticity . . . . . . . . . . . . . . 110

7 Aerodynamics Solver: Panel Code $\quad 115$

7.1 Fundamentals of Panel Codes . . . . . . . . . . . . . . . . 115

7.2 Theory . . . . . . . . . . . . . . . . . . . . . . . 116

7.3 Extension to 3D . . . . . . . . . . . . . . . . . . . . . . . . . 117

7.3 .1 Panel Coordinate Transformation . . . . . . . . . . . . 117

7.4 APAME Panel Code . . . . . . . . . . . . . . . . . . . . . . 120

7.4 .1 Boundary Conditions . . . . . . . . . . . . . . . . . . 121

7.4 .2 Geometry Validation . . . . . . . . . . . . . . . . . 126

7.4 .3 Field Velocity Survey . . . . . . . . . . . . . . . . . . . . 127

7.4 .4 Streamlines $\ldots \ldots \ldots \ldots \ldots \ldots$

7.4 .5 Surface Pressure Coefficient Interpolation. . . . . . . . . . 130

7.5 Summary of Contributions to APAME $\ldots \ldots \ldots \ldots \ldots$

8 Panel Code-Particle Scheme Integration 134

8.1 Panel Influence on Particles . . . . . . . . . . . . . . . . . . 135

8.1 .1 Particle Strength Update Equation Verification ... . . . . . 136

8.2 Particle Influence on Panels $\ldots \ldots \ldots \ldots \ldots$

$8.3 \quad$ Steps To Get A Solution . . . . . . . . . . . . . . . . . . . . . . . 139

9 AGARD Wind Tunnel Test 140

9.1 Wind Tunnel Test Information . . . . . . . . . . . . . . . . . . . . 140

9.1.1 AGARD Experiment Shortcomings and Differences . . . . . . 144

9.2 Configuration 1 Panel Code Calibration . . . . . . . . . . . . . 148

9.2 .1 Surface Pressure Coefficient Measurements . . . . . . . . . . 148

9.3 Configuration 2 Panel Code Calibration. . . . . . . . . . . . . . 149

9.3 .1 Surface Pressure Coefficient Measurements . . . . . . . . . . 152

$9.3 .2 \quad$ Wing Panel Density Study . . . . . . . . . . . . . . 156

10 Propeller-Airframe Interaction Simulations 164

10.1 Propeller Distribution Matching . . . . . . . . . . . . . . . . 165

10.1 .1 Axial Velocity Distribution Matching . . . . . . . . . . . 165

10.1 .2 Swirl Velocity Distribution Matching _ . . . . . . . . . 166

10.2 AGARD Configuration $1 \ldots \ldots \ldots \ldots$

10.2 .1 Resultant Pseudo-Steady Flowfield . . . . . . . . . . . 168

10.2 .2 Velocity Profile Comparisons . . . . . . . . . . . . . . . 171

10.2 .3 Nacelle Pressure Coefficients . . . . . . . . . . . . . . . . 173

10.3 AGARD Configuration $2 \ldots \ldots \ldots \ldots \ldots \ldots$

10.3 .1 Resultant Pseudo-Steady Flowfield . . . . . . . . . . . . 174

10.3 .2 Velocity Profile Comparisons . . . . . . . . . . . . . . . . . . 179

10.3 .3 Nacelle Pressure Coefficients . . . . . . . . . . . . . . . . 181 
10.3 .4 Wing Pressure Coefficients . . . . . . . . . . . . . . . . . 182

10.4 Channel Wing Study . . . . . . . . . . . . . . . . . . . . . 186

10.4 .1 Resultant Pseudo-Steady Flowfield . . . . . . . . . . . 186

10.4 .2 Wing Pressure Coefficient Contours . . . . . . . . . . . . . . 189

$\begin{array}{ll}11 \text { Conclusions } & 191\end{array}$

11.1 Limitations $\ldots \ldots \ldots$

11.2 Future Work . . . . . . . . . . . . . . . . . . . . . . 193

\begin{tabular}{ll}
\hline Appendices & 208
\end{tabular}

\begin{tabular}{ll}
\hline A Particle Cell Area Derivation & 208
\end{tabular}

\begin{tabular}{ll}
\hline B Thrust Coefficient Derivation & 210
\end{tabular}

B.1 Iterative Coefficient Solution . . . . . . . . . . . . . . . . . . . 212

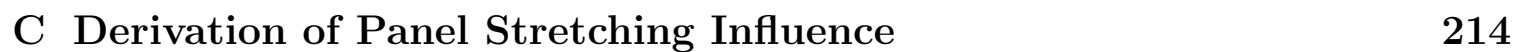

C.1 Transpose Scheme. . . . . . . . . . . . . . . . . . . . . . . . . . . 215

C.1.1 Point Source. . . . . . . . . . . . . . . . . . . . 217

C.1.2 Point Doublet . . . . . . . . . . . . . . . . . . . . 221

C.1.3 Constant Strength Source . . . . . . . . . . . . . . . . 227

C.1.4 Constant Strength Doublet. . . . . . . . . . . . . . . . 236

\begin{tabular}{ll}
\hline D Panel Influence Verification & 240
\end{tabular}

D.1 Panel Potential . . . . . . . . . . . . . . . . . . . . . . . . . . . . . 243

D.1.1 Point Doublet . . . . . . . . . . . . . . . . . . . . . . 244

D.1.2 Point Source. . . . . . . . . . . . . . . . . . . . . . . 245

D.1.3 Constant Strength Doublet. . . . . . . . . . . . . . . . . . 246

D.1.4 Constant Strength Source . . . . . . . . . . . . . . . . . 248

D.2 Panel Velocity . . . . . . . . . . . . . . . . . . . . . . . . . . . 250

D.2.1 Point Source. . . . . . . . . . . . . . . . . . . . . 252

D.2.2 Point Doublet . . . . . . . . . . . . . . . . . . . . . . . . 252

D.2.3 Constant Strength Source _. . . . . . . . . . . . . . . 253

D.2.4 Constant Strength Doublet. . . . . . . . . . . . . . . . . 254

D.3 Velocity Gradient . . . . . . . . . . . . . . . . . . . . . . . . 256

D.4 Distribution Comparisons . . . . . . . . . . . . . . . . . 257

D.5 Vortex Particle Stretching . . . . . . . . . . . . . . . . . . . . 262 


\section{List of Tables}

$2.1 \quad$ Steps for a FASTAERO panel code solution with a vortex particle wake.1 10

2.2 Computation time using various propeller interference prediction meth-

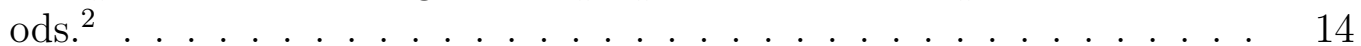

4.1 Comparison of current vortex particle implementation with Winckelmans initial conditions 13 . . . . . . . . . . . . . . 54

8.1 Steps to generate a particle-panel code solution for propeller-airframe interaction. . . . . . . . . . . . . . . . . . . . . . 139 


\section{List of Figures}

2.1 Vortex particle simulation of flow around the Neath Viaduct. 4 . . . . 8

2.2 FASTAERO panel method solution with a vortex particle wake.1 ${ }^{15}$. . 9

2.3 GENUVP simulation of a vortex particle 3D rotor wake. 6 . . . . . . . 12

2.4 Panel distribution for an AGARD experimental configuration created by Lotstedt.7 . . . . . . . . . . . . . . . . 17

2.5 Helical trailing vortex panels from Yang, Li, and E..8 . . . . . . . 20

3.1 A comparison of a singular particle kernel with a regularized particle

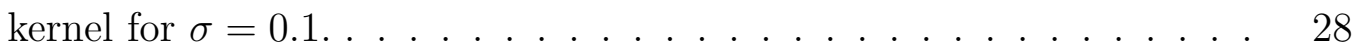

4.1 Discretization of layers of particles into a two dimensional disk with

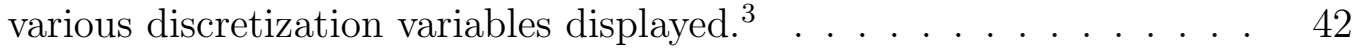

4.2 Vortex ring modeled by 6,480 particles from an isometric view as well as from a top view, with the top view showing the spacing between each particle disk. . . . . . . . . . . . . . . . . . . . . 43

4.3 Vortex particle strength vectors for all particles in a single disk of the vortex ring. . . . . . . . . . . . . . . . . . . 45

4.4 Convergence of particle strength vectors with Beale's Method iteration

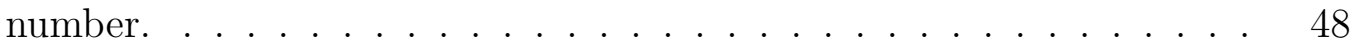

4.5 Error associated with a third order Runge-Kutta approximation of the sine function, both with and without round-off error removal. . . . . . 52

4.6 Time dependent decay of (a) total vorticity, and (b) angular impulse using a particle discretization of a vortex ring. . . . . . . . . . . . . . 57

$4.7 \quad$ Time dependent linear impulse decay using a vortex particle discretization of a vortex ring. . . . . . . . . . . . . . . . . 58

4.8 Time dependent enstrophy decay using a vortex particle discretization of a vortex ring. . . . . . . . . . . . . . . . . . . . 58

4.9 Time dependent kinetic energy decay using a vortex particle discretization of a vortex ring. . . . . . . . . . . . . . . . 59

4.10 Contours of vorticity for both (a) the initial particle discretization of the ring and (b) the final solution at $t=5$ seconds. $\ldots . . . . .60$ 
5.1 Radial variation of radial velocity at several axial locations (symmetric about $\mathrm{z}=0$ ). . . . . . . . . . . . . . . . . . . . 68

5.2 Radial variation of axial velocity at several axial locations. . . . . . . 69

5.3 Radial variation of radial velocity at several axial locations (symmetric about $\mathrm{z}=0$ ). . . . . . . . . . . . . . . . . . 71

5.4 Radial variation of axial velocity at several axial locations. . . . . . . 72

5.5 Radial variation of radial velocity at several axial locations. . . . . . . . 75

5.6 Radial variation of axial velocity at several axial locations. . . . . . . 76

$5.7 \Delta C_{p}$ jump for the AGARD experiment (exp.) along with calculated

$\Delta C_{p}$ at two different efficiency levels, actuator disk efficiency (AD) and propeller efficiency (p), and error from experimental values. . . . . . . 82

$6.1 \quad$ Discretization of particles into a two dimensional disk ${ }^{3}$. . . . . . . . 87

6.2 A sample particle streamtube with unsteady wake roll up occurring. . 90

6.3 Vorticity across the diameter of the actuator disk for (a) $n=225$ particles per disk and (b) $n=1,521$ particles per disk. . . . . . . 93

6.4 Vorticity inside the streamtube downstream of the actuator disk for (a) $n=225$ particles per disk and (b) $n=1,521$ particles per disk. . . 94

6.5 Comparison of analytical (solid) and discretized vortex particle (dashed) axial perturbation velocities at several axial positions relative to the actuator disk for (a) $n=225$ particles per disk and (b) $n=1,521$

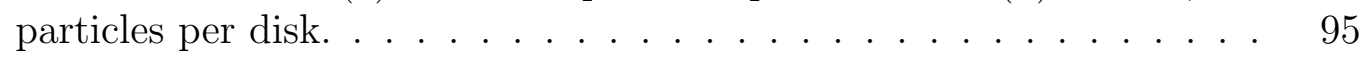

6.6 Comparison of analytical (solid) and discretized vortex particle (dashed) radial perturbation velocities at several axial positions relative to the actuator disk for (a) $n=225$ particles per disk and (b) $n=1,521$ particles per disk. . . . . . . . . . . . . . . . . . . . . 96

6.7 Final particle locations after convection for (a) $n=225$ particles per disk and (b) $n=1,521$ particles per disk. . . . . . . . . . . . 97

6.8 Vorticity across the diameter of the actuator disk for (a) $n=225$

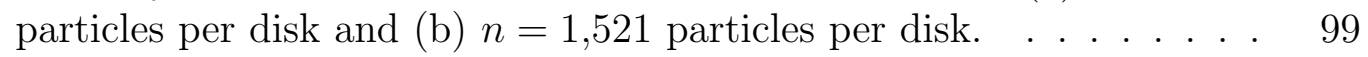

6.9 Vorticity inside the streamtube downstream of the actuator disk for (a) $n=225$ particles per disk and (b) $n=1,521$ particles per disk. . . 100

6.10 Comparison of analytical (solid) and discretized vortex particle (dashed) axial perturbation velocities at several axial positions relative to the actuator disk for (a) $n=225$ particles per disk and (b) $n=1,521$ particles per disk. ...................... 101

6.11 Comparison of analytical (solid) and discretized vortex particle (dashed) radial perturbation velocities at several axial positions relative to the actuator disk for (a) $n=225$ particles per disk and (b) $n=1,521$ particles per disk. . . . . . . . . . . . . . . . 101

6.12 Vorticity across the diameter of the actuator disk for (a) $n=225$ particles per disk and (b) $n=1,521$ particles per disk. . . . . . . 103 
6.13 Vorticity inside the streamtube downstream of the actuator disk for (a) $n=225$ particles per disk and (b) $n=1,521$ particles per disk. . . 104

6.14 Comparison of analytical (solid) and discretized vortex particle (dashed) axial perturbation velocities at several axial positions relative to the actuator disk for (a) $n=225$ particles per disk and (b) $n=1,521$ particles per disk. . . . . . . . . . . . . . . . . . . . . . . . . . . . . 104

6.15 Comparison of analytical (solid) and discretized vortex particle (dashed) radial perturbation velocities at several axial positions relative to the actuator disk for (a) $n=225$ particles per disk and (b) $n=1,521$ particles per disk. . . . . . . . . . . . . . . . . . . . . . . . 105

6.16 Vorticity across the diameter of the actuator disk for (a) $n=225$

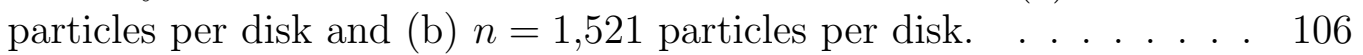

6.17 Vorticity inside the streamtube downstream of the actuator disk for (a) $n=225$ particles per disk and (b) $n=1,521$ particles per disk. . . 108

6.18 Comparison of analytical (solid) and discretized vortex particle (dashed) axial perturbation velocities at several axial positions relative to the actuator disk for (a) $n=225$ particles per disk and (b) $n=1,521$ particles per disk. . . . . . . . . . . . . . . . . . . . . . . . . . . . . . 109

6.19 Comparison of analytical (solid) and discretized vortex particle (dashed) radial perturbation velocities at several axial positions relative to the actuator disk for (a) $n=225$ particles per disk and (b) $n=1,521$ particles per disk. . . . . . . . . . . . . . . . . . . . . . . . . . . . 109

6.20 Vorticity across the diameter of the actuator disk for (a) halved and (b) quartered time step size. . . . . . . . . . . . . . . 111

6.21 Vorticity inside the streamtube downstream of the actuator disk for (a) halved and (b) quartered time step size. . . . . . . . . . . . . . . 112

6.22 Comparison of analytical (solid) and discretized vortex particle (dashed) axial perturbation velocities at several axial positions relative to the actuator disk for (a) halved and (b) quartered time step size. . . . . . 113

6.23 Comparison of analytical (solid) and discretized vortex particle (dashed) radial perturbation velocities at several axial positions relative to the actuator disk for (a) halved and (b) quartered time step size. . . . . . 114

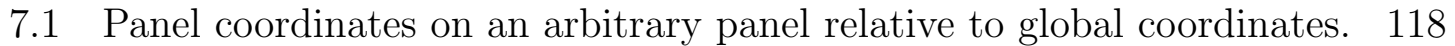

7.2 An example of a distribution with inward facing normal vectors (dark blue) on the wing in the upper left and correct outward normal vectors (teal) on the wing in the bottom right. . . . . . . . . . . . . . . . . . 127

7.3 An example of streamlines generated using the MATLAB function. . 129

7.4 A 2D example of streamlines generated from the time stepping technique around a 3D symmetric wing panel solution. . . . . . . . . . . . 131 
8.1 Example of 9 survey points relative to the single constant strength source panel, as well as the velocity vectors corresponding to each point. ${ }^{9} 137$

9.1 The four unique configurations from AGARD wind tunnel tests. 9 . . 141

9.2 Configuration 3 model size relative to the wind tunnel ${ }^{9} \mid \ldots \ldots$. . . . . 143

9.3 Paneled model of AGARD Configuration 1 with pressure port locations shown as black dots. . . . . . . . . . . . . . . . . . . . 149

9.4 Comparison of Configuration 1 experimentally measured pressure coefficients at nacelle pressure ports with computationally calculated values from the APAME panel code. . . . . . . . . . . . . . . . . 150

9.5 Comparison of Configuration 1 experimentally measured pressure coefficients at nacelle pressure ports with computationally calculated values from the APAME panel code. . . . . . . . . . . . . . . . . . . 151

9.6 Paneled model of AGARD Configuration 2 with pressure port locations shown as black dots. . . . . . . . . . . . . . . . . . . . 152

9.7 Comparison of Configuration 2 experimentally measured pressure coefficients at nacelle pressure ports with computationally calculated values from the APAME panel code. . . . . . . . . . . . . . . . . . . . . . 154

9.8 Comparison of Configuration 2 experimentally measured pressure coefficients at nacelle pressure ports with computationally calculated values from the APAME panel code. . . . . . . . . . . . . . . . . . . . . . . 155

9.9 Configuration 2 model created in $\mathrm{PRO} / \mathrm{E} \cdot 9$. . . . . . . . . . . . . . . 157

9.10 Side view of edge association in ICEM. . . . . . . . . . . . . . . . . . 159

9.11 Top view of edge association in ICEM. . . . . . . . . . . . . . . . . 159

9.12 Iso view of separate blocks in geometry in ICEM, scaled to show clear separation between blocks. . . . . . . . . . . . . . . . . . . 160

9.13 Comparison of Configuration 2 experimentally measured pressure coefficients $\left(^{*}\right)$ at wing pressure ports with computationally calculated values (-) from the APAME panel code. . . . . . . . . . . . . . 163

10.1 Fully convected particle locations around Configuration 1 at the end of 33 time steps. . . . . . . . . . . . . . . . . . . . . . . 169

10.2 Aerodynamic coefficients for Configuration 1 with and without stretching turned on. . . . . . . . . . . . . . . . 170

10.3 Velocity profiles at $x=10 \mathrm{~mm}$, close behind the actuator disk for Configuration 1. . . . . . . . . . . . . . . 172

10.4 Velocity profiles at $x=525 \mathrm{~mm}$, around the middle of the nacelle for Configuration 1. . . . . . . . . . . . . . . . . 172

10.5 Velocity profiles at $x=925 \mathrm{~mm}$, near the end of the nacelle for Configuration 1. . . . . . . . . . . . . . . . 173 
10.6 Comparison of Configuration 1 experimentally measured pressure coefficients at nacelle pressure ports with computationally calculated values from the APAME panel code with the propeller on. . . . . . . . . . . 175

10.7 Comparison of Configuration 1 experimentally measured pressure coefficients at nacelle pressure ports with computationally calculated values from the APAME panel code with the propeller on. . . . . . . . . . . 176

10.8 Fully convected particle locations around Configuration 2 at the end of 31 time steps. . . . . . . . . . . . . . . . . . . . . . . . 177

10.9 Fully convected particle locations around Configuration 2 at the end of 31 time steps, with the positive $V_{\phi}$ direction indicated, where the

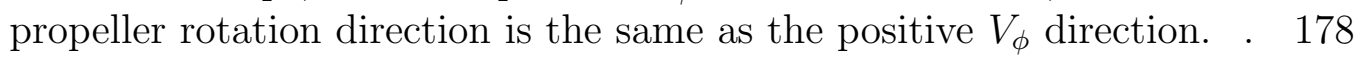

10.10Aerodynamic coefficients for Configuration 2 without stretching turned

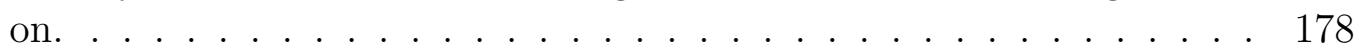

10.11Velocity profiles at $x=10 \mathrm{~mm}$, close behind the actuator disk for Configuration 2. . . . . . . . . . . . . . . . . . 179

10.12 Velocity profiles at $x=525 \mathrm{~mm}$, around the middle of the nacelle for Configuration 2. . . . . . . . . . . . . . . . . . . . . . . . 180

10.13 Velocity profiles at $x=925 \mathrm{~mm}$, near the end of the nacelle for Configuration 2. . . . . . . . . . . . . . . . . . . . . 181

10.14Comparison of Configuration 2 experimentally measured pressure coefficients at nacelle pressure ports with computationally calculated values from the APAME panel code with the propeller on. . . . . . . . . . . 183

10.15Comparison of Configuration 2 experimentally measured pressure coefficients at nacelle pressure ports with computationally calculated values from the APAME panel code with the propeller on. . . . . . . . . . . 184

10.16Comparison of Configuration 2 experimentally measured pressure coefficients at wing pressure ports with computationally calculated values from the APAME panel code with the propeller on. . . . . . . . . . . 187

10.17Fully convected particle locations around a channel wing at the end of 25 time steps with stretching at the onset of instability. . . . . . . . . 188

10.18Aerodynamic coefficients for a channel wing configuration with and without stretching turned on. . . . . . . . . . . . . . . . . . 189

10.19Pressure coefficient distribution around a channel wing prior to activation of the actuator disk. . . . . . . . . . . . . . . . . . . . . . . 190

10.20Pressure coefficient distribution around a channel wing after 25 time steps. . . . . . . . . . . . . . . . . . 190

D.1 Survey with corresponding velocity vectors relative to arbitrarily oriented panel with a constant strength source element. . . . . . . . . . 243

D.2 Panel coordinate vectors and point of interest vector. . . . . . . . . . 245

D.3 Vectors corresponding to current panel side. . . . . . . . . . . . . . . 249 
D.4 Survey paths relative to the panel, with surveys taken in the $z$ axis as well as along the diagonal and the median of the panel. . . . . . . . . 258

D.5 Velocity influence of point (Pt.) and constant strength (Ct.) distribution types as distance above panel varies. . . . . . . . . . . . . . 259

D.6 Difference between constant strength and point approximation as height above panel varies. . . . . . . . . . . . . . . . . 260

D.7 Velocity influence of different distributions as distance along panel diagonal varies. . . . . . . . . . . . . . . . . . . . 261

D.8 Velocity influence of different distributions as distance along panel median varies. . . . . . . . . . . . . . . 262 


\title{
Nomenclature
}

\author{
General \\ APAME Aircraft PAnel MEethod \\ CFD Computational fluid dynamics \\ $C_{p} \quad$ Pressure coefficient \\ $C_{T} \quad$ Thrust Coefficient \\ I Identity matrix \\ $\mathscr{O} \quad$ Order of error \\ $P \quad$ Pressure \\ Q Velocity \\ Re Reynold's number \\ $S \quad$ Reference area \\ $T \quad$ Thrust \\ U Velocity \\ V Velocity \\ $b_{\#} \quad$ Low storage Runge-Kutta scaling coefficient \\ $e_{\#} \quad$ Low storage Runge-Kutta roundoff error \\ $f(x) \quad$ Generic function \\ $\tilde{h} \quad$ Low storage Runge-Kutta step size \\ $k_{\#} \quad$ Traditional Runge-Kutta coefficient \\ $p_{o} \quad$ Total pressure \\ $q \quad$ Dynamic pressure \\ $q_{\#} \quad$ Low storage Runge-Kutta coefficient \\ $t \quad$ Time \\ u Global velocity vector \\ $\mathbf{u}^{\prime} \quad$ Local velocity vector \\ $(u, v, w) \quad$ Global velocity components \\ $\mathrm{x} \quad$ Position vector \\ $\mathbf{x}^{\prime} \quad$ Local position vector \\ $(x, y, z) \quad$ Coordinate components \\ $\nabla \quad$ Del operator \\ $\rho \quad$ Density \\ $\Delta \quad$ Finite difference step size
}




$\begin{array}{ll}\text { Vortex } & \text { Particle Theory } \\ A & \text { Beale's Method matrix } \\ \mathbf{A} & \text { Angular impulse } \\ E & \text { Kinetic energy } \\ E(k) & \text { Complete elliptic integral of the second kind } \\ \tilde{E} & \text { Particle approximation of kinetic energy } \\ G(\mathbf{x}) & \text { Green's function } \\ \mathscr{H} & \text { Helicity } \\ \mathbf{I} & \text { Linear impulse } \\ \mathbf{K} & \text { Biot-Savart Kernel } \\ N_{s} & \text { Number of particles in a disk } \\ N_{\phi} & \text { Number of disks discretizing a vortex ring } \\ R & \text { Radius of a vortex ring (Winckelmans) } \\ h & \text { Typical distance between neighboring particles } \\ n_{c} & \text { Number of additional layers in a vortex disk } \\ q(x) & \text { Regularization function for velocity } \\ r_{1} & \text { Radius to cell lower bound } \\ r_{2} & \text { Radius to cell upper bound } \\ r_{c} & \text { Cell area centroid radius } \\ r_{l} & \text { Cell radius } \\ v o l & \text { Particle volume } \\ \boldsymbol{\alpha} & \text { Particle strength vector } \\ \delta & \text { Dirac delta function } \\ \varepsilon & \text { Enstrophy } \\ \tilde{\varepsilon} & \text { Particle approximation of enstrophy } \\ \eta & \text { Approximation to kernel for heat equation } \\ \omega & \text { Vorticity vector } \\ \omega_{x} & \text { Axial component of the vorticity, responsible for swirl velocity } \\ \tilde{\boldsymbol{\omega}}(\mathbf{x}, t) & \text { Particle approximation of vorticity field } \\ \phi & \text { Azimuthal vortex ring coordinate } \\ \rho & \text { Dimensionless radial distance between particles, }\left|\mathbf{x}^{p}-\mathbf{x}^{q}\right| / \sigma \\ \sigma & \text { Vortex core radius } \\ \theta_{1} & \text { Left boundary of particle cell } \\ \theta_{2} & \text { Right boundary of particle cell } \\ \nu & \text { Kinematic viscosity } \\ \zeta & \text { Regularization function for Dirac delta } \\ \Gamma & \text { Vortex ring circulation } \\ \Omega & \text { Total vorticity } \\ \Psi & \text { Streamfunction } \\ \tilde{\Psi} & \text { Particle approximation of the streamfunction } \\ & \end{array}$




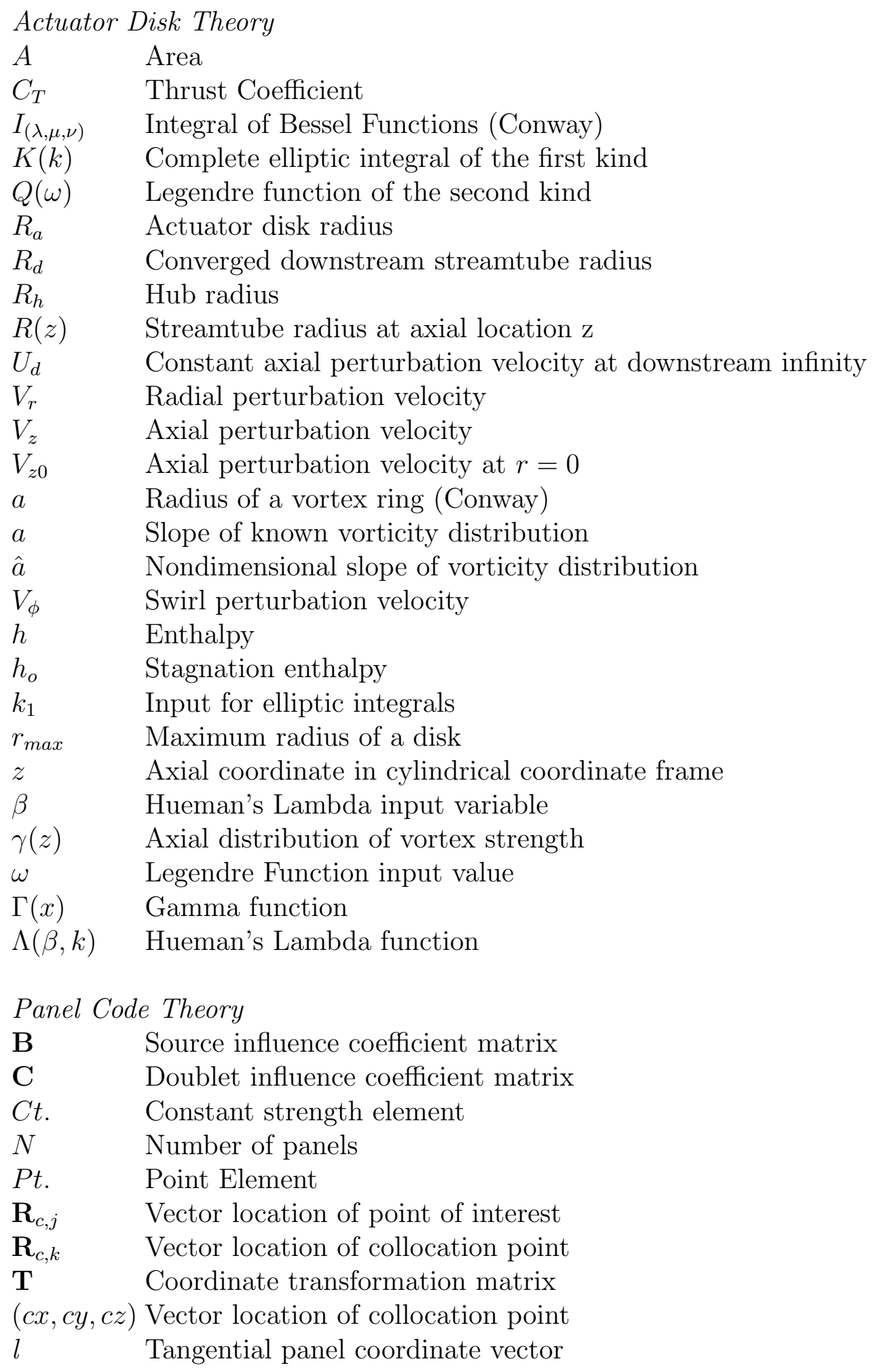




$\begin{array}{ll}m & \text { Parallel panel coordinate vector } \\ n & \text { Normal panel coordinate vector } \\ \left(x_{0}, y_{0}, z_{0}\right) & \text { Influencing element position components } \\ \left(x^{\prime}, y^{\prime}, z^{\prime}\right) & \text { Local coordinate components } \\ \mu & \text { Doublet strength } \\ \sigma & \text { Source strength } \\ \Phi & \text { Potential } \\ & \\ \text { Subscript } & \\ A & \text { Summed influence from all actuator disks } \\ R & \text { Applying to the vortex ring } \\ d & \text { Doublet } \\ d i s k & \text { Of or relating to the actuator disk } \\ f & \text { Non divergence-free } \\ \text { global } & \text { Global coordinate frame } \\ i & \text { Panel side index } \\ \text { in } & \text { Inside of panel boundary } \\ j & \text { Panel index } \\ \text { panel } & \text { Panel coordinate frame } \\ s & \text { Source } \\ \infty & \text { Freestream condition } \\ * & \text { limiting value just outside paneled boundary } \\ \sigma & \text { Regularized version } \\ \theta & \text { Applying to the } \theta \text { coordinate axis } \\ \text { Superscript } & \\ N & \text { Divergence free } \\ T & \text { Transpose } \\ q & \text { Particle index } \\ & \text { Particle index (secondary) } \\ & \end{array}$




\section{Chapter 1}

\section{Introduction}

Regardless of discipline, the early stages of design are often tumultuous, turbulent times. To find the optimum answer to a given design problem it is necessary to evaluate many fundamentally different potential solutions, and this analysis must be done rapidly and with minimal computing and manpower. In the field of aerodynamics, CFD solutions can provide extremely high fidelity solutions to complex flow problems, but these solutions necessitate a volume grid that can be quite expensive to generate and extremely geometry dependent. The panel code, on the other hand, provides a useful tool for preliminary design, where lower fidelity results are acceptable, but the flexibility to rapidly analyze widely varying geometries is essential.

The typical panel method, while fast compared with CFD, is limited in its ability to account for the effect of work done on the flow. While this is not a serious problem in many aerodynamic simulations, in certain cases it can be quite handicapping, such as when a propeller is present, especially when those effects produce asymmetric forces and moments on the vehicle. A tool capable of providing the flexibility and 
speed necessary for initial design, while still providing the ability to account for the benefits and penalties carried with propulsion interaction effects, would be extremely valuable to the design community.

\section{$1.1 \quad$ Approach}

This research focuses on a way to overcome the deficiency of the panel code rooted in the inability to account for vorticity through the addition of vortex particles. The vortex particles are used to discretize and numerically simulate an actuator disk. This actuator disk simulation provides a time averaged, pseudo-steady state solution to the propeller effects, which can be combined with the solution from the panel code to provide insight into the qualitative and quantitative effects of propeller or engine slipstreams on the aerodynamic performance of various geometries.

This method could prove quite useful in assessing the value of more unconventional configurations such as channel wing aircraft, or asymmetric aircraft, whose design may depend on asymmetric loading from propellers to achieve steady flight. Additionally, any design which may potentially achieve incremental lift improvements due to blown surfaces would benefit greatly from the ability to account for those improvements from the initial stages of design. Accounting for the more detailed features of the design early on allows for a wider design space with more potential solutions to the problem, and correspondingly more potential chances to find the best global optimum. 


\subsubsection{Crocco's Theorem}

Another, more visual way to understand the goal of this research is to examine Crocco's Theorem, which can be written as

$$
\mathbf{V} \times \boldsymbol{\omega}=\frac{1}{\rho} \nabla p_{o}
$$

for a steady, incompressible, inviscid fluid flow ${ }^{10}$ A traditional panel code requires the additional assumption that the flow is irrotational, which consequently means vorticity won't be modeled. Examining Equation 1.1 it is clear that this assumption forces the entire left hand side to become zero. With the left hand side equal to zero, the right hand side must necessarily be zero. Without the ability to capture changes in stagnation pressure, a panel code cannot hope to account for the stagnation pressure changes associated with a propeller streamtube.

The addition of vortex particles to a panel code provides a way to account for the left hand side of Crocco's Theorem, through the addition of vorticity. The vortex particles create a nonzero vorticity field, which provides a nonzero value to $\boldsymbol{\omega}$, allowing the right hand side of Equation 1.1 to remain nonzero. Thus, variation in stagnation pressure can be captured with variation in vorticity.

\subsection{Document Roadmap}

This document traverses through various independent topics and shows how they can be woven together to create a powerful tool useful to the conceptual designer. Now that the problem of capturing complex aerodynamic interactions has been explained, a 
brief summary of prior methods that attempt to model those interactions is provided, along with their primary differences from the current method, in Chapter 2.

The method has two major parts, which can be thought about in two ways. To capture the desired interference effects it is necessary to have both a propeller model and an aerodynamics solver. Similarly, the model requires both a rotational model to capture slipstream effects, and a simple irrotational model to define the rest of the flowfield. The rotational propeller flowfield was modeled using a discrete regularized vortex particle scheme to represent a time averaged actuator disk. The irrotational aerodynamic solver selected was the panel code, for which APAME, a three-dimensional constant strength source-doublet panel code, contributed the brunt of the work.

The two separate models will be examined consecutively, starting with the rotational model composed of the vortex particle scheme in Chapter 3 and Chapter 4. Once the vortex particle scheme has been demonstrated, the particles will then be applied in a manner consistent with actuator disk theory, which is detailed in Chapter 5 and Chapter 6. Once the propeller model has been defined, the APAME panel code will then be discussed in Chapter 7 along with the author's contributions to it. With the two components established, the communication and interaction associated with their integration will be explained in Chapter 8. As a final validation of the model, a series of AGARD wind tunnel tests are introduced in Chapter 9, which were conducted as a baseline to which computational simulations could be compared. The 
results of the current method relative to those AGARD experiments will be presented as a final validation of the method in Chapter 10 , followed by concluding remarks in Chapter 11 . 


\section{Chapter 2}

\section{Comparison with Historical Methods}

In order to understand the applicability of the proposed method, it was necessary to understand the current state of computational techniques for propeller-airframe interaction. An extensive investigation of existing methods was launched to learn the lessons of where other techniques fell short, and what potential improvements could be made with the proposed research.

As vortex particle theory played a major part in the proposed work, an additional study was conducted to ascertain information on the various ways vortex particle techniques are currently used to understand if our proposal was indeed feasible. Additionally, the current propeller-airframe interaction methods were examined based upon the different propeller models each method employed. For many of the propeller models a variety of different aerodynamic solvers were used by different researchers. 


\subsection{Current Applications of Vortex Particles}

Vortex particle methods are currently used for a variety of applications that fall under the discipline of aerodynamics. Most frequently, the particles are used to represent the wakes shed from various aerodynamic bodies without the use of panels or grids. In panel codes wake panels can be problematic, especially in areas of high vorticity, because the panels can become crossed or overlapped in very nonphysical ways. The benefit of vortex particles it that there is no connectivity data in the sense that there is for a paneled wake, and therefore there is minimal numerical danger with wake roll up and, with the correct time step treatment, it is virtually impossible for two particles to overlap. Particles are also used in applications outside of aircraft, such as the simulation of aerodynamic loads on bridges and buildings.

\subsubsection{Structural Aerodynamics Using Vortex Particles}

Morgentha ${ }^{\sqrt{4}}$ uses a grid free vortex particle method to discretize the Navier-Stokes equations in order to conduct two dimensional studies of the aerodynamics of various buildings and structures. The vortex particle method provided a technique to analyze the flow around complex geometries, which provided a powerful numerical tool useful as either a companion or competitor to wind tunnel experiments. Morgenthal also created an algorithm to achieve highly efficient computation of particle velocities, allowing for high particle resolutions at reasonable computational cost. A sample solution from his work is shown in Figure 2.1. 


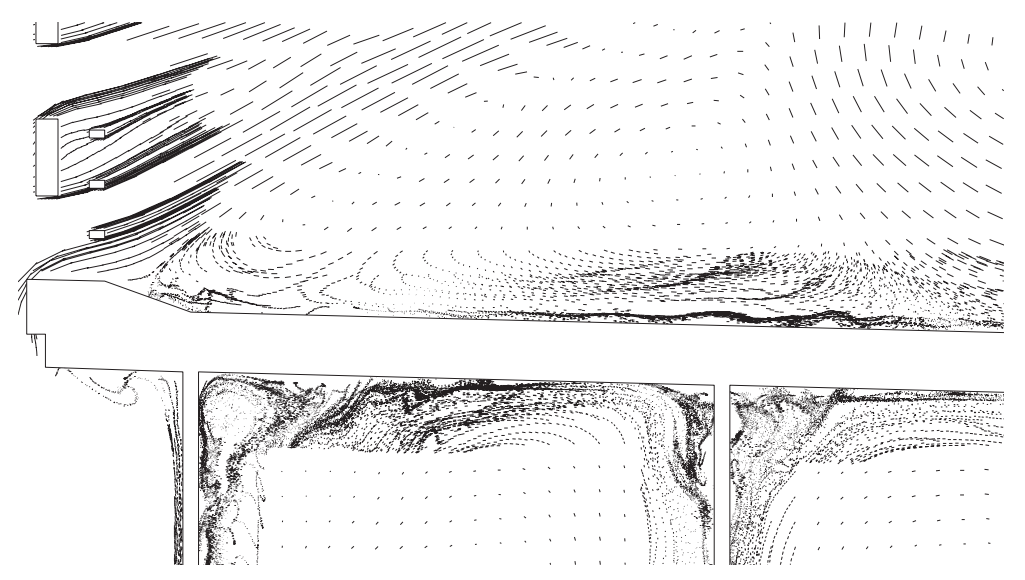

Figure 2.1: Vortex particle simulation of flow around the Neath Viaduct!

The particle scheme was used to analyze flow around arbitrary shapes such as cylinders and prisms in a flowfield, and then advanced to more difficult geometries, including existing structures such as the Neath Viaduct and the Glasgow Wing Tower. The particle scheme was both accurate and efficient, and provided good agreement with wind tunnel measurements.

\subsubsection{Vortex Particle Aircraft Wakes}

Another recent application of vortex particles is the particle aircraft wake, used to compliment a three-dimensional panel code. This example fuses the vortex particle scheme with a traditional panel code in a similar way to how the proposed research aims to, and therefore has a very similar infrastructure, thus demonstrating that integration of the two methods is in fact possible. This use of vortex particles is possible because the wake introduces circulation to the flowfield, which must be present to model lift. Circulation is directly related to vorticity through $\Gamma=\int_{S} \boldsymbol{\omega} \cdot \mathbf{n} d S$.

The vortex particle wake has several advantages over traditional panel wakes. The 
particles don't have any connectivity data, and so each individual particle is free to move independently of the other particles. This is especially useful in areas of high vorticity such as the wing tip vortices on an aircraft. Also, the point particle, rather than a panel with an associated area, cannot intersect another particle. Additionally, the vortex particle wake avoids the high user interactivity required in selecting wake position and wake-body intersection issues. An example of a solution of an aircraft heave from FASTAERO generated with a vortex particle wake can be seen in Figure 2.2, 11

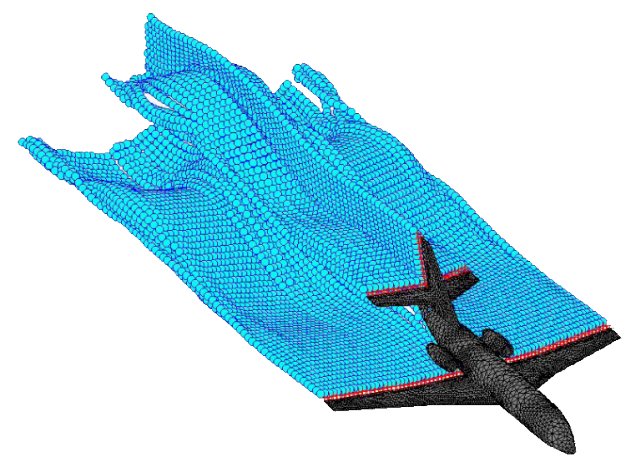

Figure 2.2: FASTAERO panel method solution with a vortex particle wake 1

The author of FASTAERO describes the steps necessary to obtain a solution in his code. These steps are shown in Table 2.1. Beyond FASTAERO additional particle methods have been used to model trailing wakes, including work by Winckelmans, $\frac{12}{12}$ and Chatelain! 13 These models are not integrated with any particular aerodynamics solver in the way that Willis incorporates a panel code in his solution, but are more narrowly focused on resolving details of the actual vortex wake behavior. 
Table 2.1: Steps for a FASTAERO panel code solution with a vortex particle wake.1

\begin{tabular}{|c|l|}
\hline Step 1 & Solve for panel strengths and wake potential jump \\
\hline Step 2 & Determine strength of vorticity to release for current time step \\
\hline Step 3 & Determine velocity and stretching for wake particles from panels \\
\hline Step 4 & Determine velocity and stretching for wake particles from particles \\
\hline Step 4a & If necessary, compute pressures and forces on the body \\
\hline Step 5 & Update particle positions in the wake \\
\hline Step 6 & Determine wake influence on body \\
\hline Step 7 & Return to Step 1 unless iteration criterion is met \\
\hline
\end{tabular}

\subsubsection{Helicopter Rotor Wakes}

In addition to using vortex particles to model the wakes shed from aircraft wings and empennage, particles likewise have been used to simulate the wake shed from rotors on helicopters $6[14$ Because the particles are still shed as a wake the method and results are quite similar to the aircraft.

Several key differences appear between the rotor modeling method of Opoku et al. $\frac{614}{14}$ and the research proposed here. First, the wake particle method releases a single particle from each wake panel at each time step. This means that depending on the number of rotor blades there will be different numbers of particles released. In the event of a two blade configuration a single line of vortex particles will be released, while a four blade rotor would release a cross of particles at each time step. This is contrary to the actuator disk in which a complete disk of particles is released each time step. This results in a key difference in that in the infinite particle limit the proposed research is azimuthally steady, while the rotor wake method is not. That is to say that at any given time in the rotor wake simulation, the rotor will be at a different 
azimuthal position, and correspondingly the solution will fluctuate with a period based around one revolution of the rotor. Additionally, the wake model requires a much higher level of detail about the geometry than the actuator disk approach. To correctly model the wake shed from a rotor, details including blade shape and count, blade incidence angles, and the chord of each blade must be accounted for. None of these details are required for an actuator disk, making it a more practical tool for early design stages where detailed information about propellers or rotors is not yet known.

Another difference between the two methods is that the rotor wake method appears to avoid placing any bodies of interest downstream of the rotor. This means that the simulation may be valuable in understanding the effects of the rotor alone, but cannot capture any interference or interaction effects between the rotor and any bodies in its path, which is a key aspect of the current research. It is not clear whether this is a product of weak boundary conditions, or simply something that has not yet been investigated. A sample of a particle rotor wake simulation can be seen in Figure 2.3.

\subsection{Propeller-Airframe Interaction History}

The modeling of interaction between aerodynamic surfaces and propulsive devices such as propellers has always been a topic of interest in the aerospace field. The effects

of a propeller on aerodynamic surfaces can often dramatically change aerodynamic 


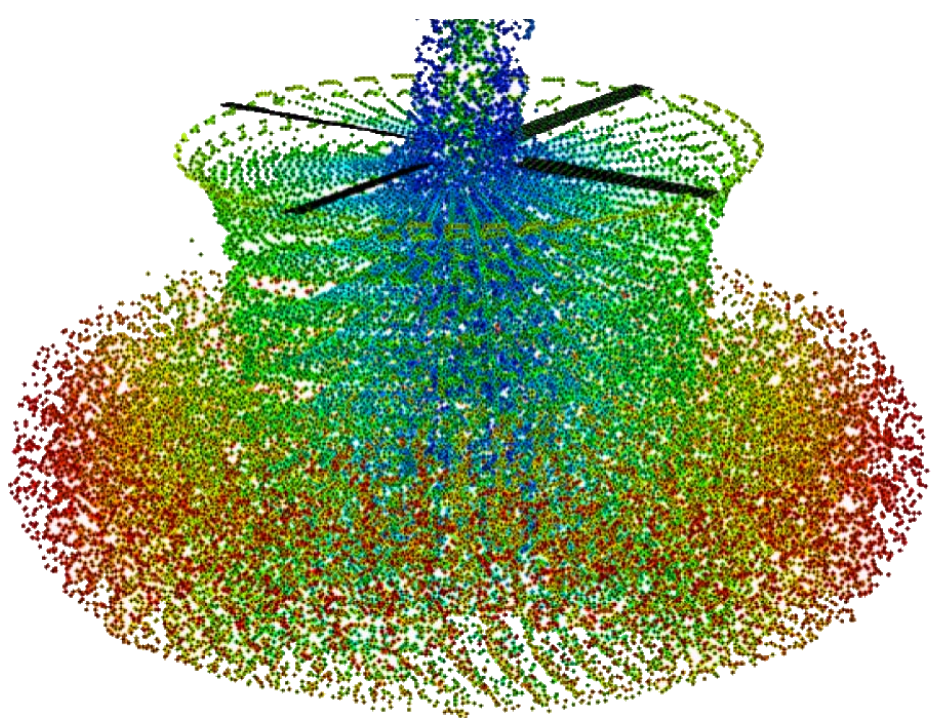

Figure 2.3: GENUVP simulation of a vortex particle 3D rotor wake ${ }^{6}$

performance. It may sometimes even be desired to account for the effects that work will have on the system, and attempt to use work effects to the benefit of the vehicle. The existing models each have their own subtleties as to the way in which the propeller is treated, as well as in which aerodynamic method is tied to the propeller model. For each of the various propeller models it is often possible to incorporate a variety of different aerodynamic solvers for interaction modeling.

\subsubsection{Actuator Disk}

By far the most frequently used propeller model in propeller-airframe interaction methods, the actuator disk model is easily applied to a large variety of different aerodynamic solvers, which means it has flexibility in terms of both level of fidelity

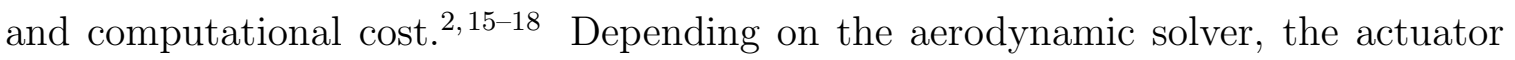
disk is implemented in varied ways, but the main theme remains that the solution 
is time averaged. The actuator disk is simply a flat plate, under the assumption of an infinite blade count, as opposed to discrete modeling of a finite number of exact blade shapes.

In 1996 Colin, Moreux, and Barillier ${ }^{22}$ presented the three interaction solvers employed by Aerospatiale, all of which use the actuator disk model. Aerospatiale intentionally employed three methods of varying fidelity, allowing for selection of the best method at various points during the design process. Each of the different fidelity levels had a different aerodynamic solver attached; the lowest was a velocity formulation panel code with Neumann boundary coditions, the middle was the commercial code MGAERO, and the final was a multiblock Euler/Navier Stokes solver called CANARI. Of the three models, only CANARI was capable of modeling feedback influence from the airframe onto the disk, through variation in the Mach number entering the actuator. With cases run on a CRAY J916, a comparison of preparation and run time is shown in Table 2.2. Both FP3D and CANARI allowed for reloading a mesh with only minor adjustments, which made minor mesh changes much quicker. Even with this feature though, it is clear that the panel code is quickest both in preparation and in computation, making it the ideal tool for the earliest stages of design.

The actuator disk model takes in inputs from one of two sources. Either data is received from propeller manufacturers or a propeller configuration is run through a lifting line code for propeller performance, and the outputs are then used as inputs for the actuator disk model. The total pressure jump associated with the disk is modeled 
based on induced velocities using a Froude approach with compressibility corrections.

Table 2.2: Computation time using various propeller interference prediction methods $\underline{2}$

\begin{tabular}{|c|c|c|}
\hline & Mesh Generation & Calculation \\
\hline FP3D & 2 days $(0.5$ days reload $)$ & 1 hour \\
\hline MGAERO & 2 days & 1 night \\
\hline CANARI & 10 days (1 to 2 days reload) & 1 to 2 nights \\
\hline
\end{tabular}

Conway ${ }^{15}$ introduces a combined actuator disk-panel method using his previously developed analytical actuator disk theory, which is detailed in Chapter 5. To integrate the analytical actuator disk method with the panel code, the time averaged velocity fields from the disk are added into the panel code as a modification to the freestream velocity. The panel code used was PMAL3D, which is a structured, constant strength source-doublet panel method. The influence is accounted for by adjusting the source strengths to be equal to $\sigma=-\left(V_{\infty}+V_{A}\right) \cdot \mathbf{n}$, where $V_{A}$ is the velocity field induced by all actuator disks present in the simulation. Conway continues on to present pressure coefficient distributions over an entire Aurora aircraft with several different propeller configurations, as well as lift, drag, and moment coefficients. No mention is made of the stagnation pressure jump occurring at this disk, nor how it was accounted for in the surface $C_{p}$ calculations downstream of the disk.

Strash and Lidnicer ${ }^{16}$ presented their own method for modeling interference effects, which incorporated an actuator disk model along with the Euler solver MGAERO, similar to the method employed by Colin et al. The flow properties of the propeller 
are used as boundary conditions on the actuator disk in the Euler code. The matching for the method appears qualitatively good, although the authors state that there wasn't enough information even from the AGARD wind tunnel test to adequately calibrate the actuator disk model. The solutions used over 900,000 grid points, requiring over 200 megabytes of storage, and approximately 6.5 hours of computing time on a Silicon Graphics R10000 machine.

Much like Strash and Lidnicer, and Colin et al., Dang integrates an Euler code with an actuator disk model to account for interaction effects $\frac{17}{17}$ Dang specifically states a desire to create an early design tool, and selects the actuator disk model from this motivation, due to the minimal requirements necessary to implement it. Dang's model assumes no radial velocity, which equates to neglecting all streamtube contraction. Additionally, the model requires the small perturbation assumption. The matching is relatively good when the required assumptions are met, although the actuator disk method produces similar results when integrated with the panel method of Hess, making the only major application of the Euler variation cases with transonic flow where a panel code is invalid! 19

Dang proposed an additional method in 1990, which utilized a similar actuator disk model, but now with a finite volume full potential code to analyze the flowfield. Dang stated that the method was both robust and efficient, even in the transonic regime, and a big step forward from his previous work in 1989 . Rizk ${ }^{20}$ proposed another transonic method using traditional finite differencing schemes alongside a small 
perturbation assumption that allows the rotational flow to be treated as potential flow even with the wing present. A final transonic prediction method was introduced by Whitfield and Jameson, 21 using an Euler equation solver, again with propeller conditions applied at a disk in the domain to represent the propeller.

Kuijvenhoven ${ }^{18}$ employs another Euler solver integrated with an actuator disk model, this time with the ability to reduce fidelity to a panel code if desired. Again the time averaged actuator disk was employed due to its minimal requirements for highly detailed propeller information, which was valuable for early design stages. The solution was developed in a sequential technique, with the propeller flowfield analyzed first without accounting for any geometry. Next a check was run to determine which portions of the geometry would be inside the slipstream, and those portions received incremental influences from the propeller flowfield. Once perturbation velocities were known, the panel code was run for a final flow solution. Kuijvenhoven claimed 10 hour run times for 1500 iterations on 110,000 cell mesh a NEC SX-2 supercomputer in 1990. This method suffers from the additional assumption of uniform inflow into the propeller, meaning influencing bodies upstream of the propeller cannot be accounted for.

\subsubsection{Lifting Line Model}

In addition to the actuator disk model, it is possible to use a lifting line model of the propeller shed vortices to obtain information about the propeller flowfield. This type of method has its roots in blade momentum theory and classical propeller theory, 
and can be integrated with a variety of aerodynamic solvers.

Lotstedt ${ }^{7}$ proposed a method for modeling interference that integrates a momentumblade element propeller model along with a panel method for aerodynamic solutions. He goes on to validate his results against the AGARD wind tunnel data that is also examined for validation in this work. $9 \sqrt{22} \mid 23$ Lotstedt's panel distribution is shown in Figure 2.4. Lotstedt emphasized that his goal in the creation of the method, similar to

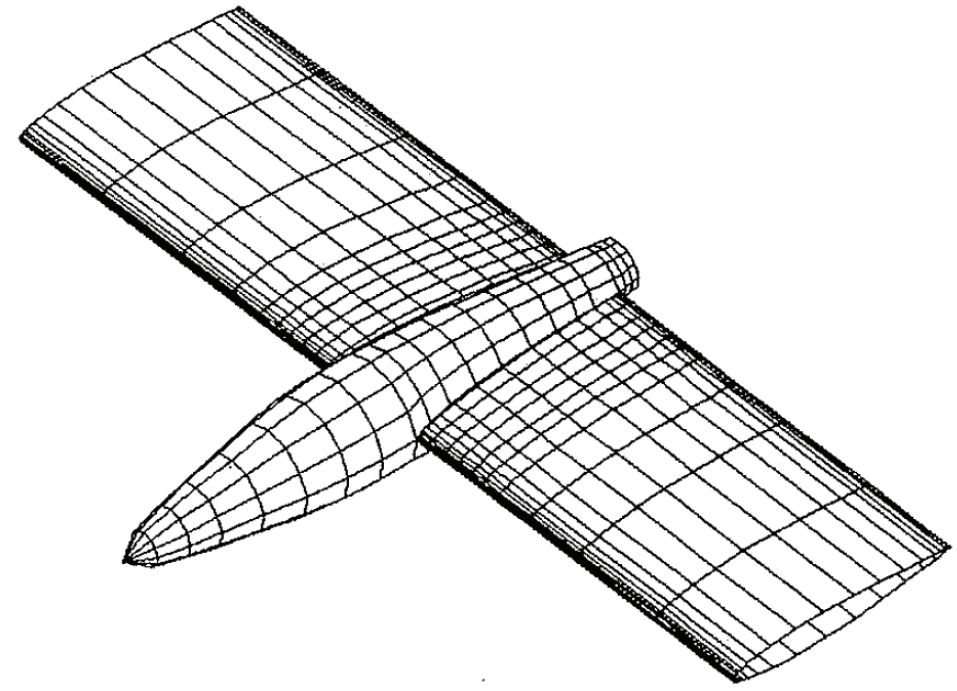

Figure 2.4: Panel distribution for an AGARD experimental configuration created by Lotstedt 7

the motivation for this work, was rooted in maintaining the low computational costs of a panel code while gaining the ability to model propeller effects. The propeller model required as an input the number of blades, the geometry, and the speed of revolution. Additionally, the slipstream was built while accounting for the nacelle geometry to some degree. Although the slipstream was constructed around the nacelle, there was no adjustment for the wing present in simulations. To generate a solution, 
streamlines were traced out of the propeller to determine the slipstream structure, and then the slipstream was broken into field panels. This required an assumption that no streamlines from the propeller entered the geometry, which necessitated deformation of geometries when streamline entry did occur. The total pressure jump at the propeller was accounted for in the streamline tracing through the slipstream. The method claimed only a $20 \%$ increase in computational time with the inclusion of the slipstream over a simple paneled geometry, with quantitatively accurate results.

Fratello, Favier, and Maresca ${ }^{24}$ provided an additional method using a lifting line model for propeller effects. The method used a free wake analysis code called SMEHEL to find the flowfield of an isolated propeller, and then imposed those influences on a panel code. As with many of these methods, the effects of the wing on the propeller wake were not accounted for in this method. Witkowski, Lee, and Sullivan also employed a vortex lattice method for their propeller model, although they only examined the simplified case of a contra-rotating propeller system.

\subsubsection{Rotating CFD grid}

Much more complex than the actuator disk or lifting line propeller models, it is also possible to use a rotating grid in a CFD solver to determine propeller influences. In 2006 Stuermer ${ }^{26}$ presented his analysis of interference effects using an unstructured Euler and Navier-Stokes code called DLR-TAU. DLR-TAU incorporated a moving Chimera grid around the propeller with a stationary grid around the rest of the geometry. To ensure adequate capture of slistpream effects, high resolution of 
cells was required everywhere in the wake. This density increase resulted in a grid of approximately 3.8 million cells for an inviscid solution around a symmetric nacelle, and 8.4 million for a viscous solution. The total run times were stated as between 100 and 250 hours on a 32 processor cluster. The agreement between experimental AGARD results and the computational method appeared quite good, although the preparation and computational cost were high. Stuermer notes that one of the major effects of the propeller was the local change in the angle of attack within the slipstream.

\subsubsection{Paneled Propeller Method}

Similar to the rotating CFD grid around the propeller geometry or particle wake, a rotating panel method is also an option. Yang, $\mathrm{Li}$, and $\mathrm{E}^{8}$ created a method utilizing just such a technique, with a paneled geometry with horseshoe vortices, as well as a paneled propeller with a rotating vortex system for its blades. An example of a paneled trailing vortex system from Yang, Li, and $\mathrm{E}$ is shown in Figure 2.5. Free wake analysis was used on the wake shed from the isolated paneled propeller. The influences of the propeller flowfield and the geometry flowfield were then superimposed to find interference effects. The propeller model did account for slipstream contraction, as well as iteratively accounting for geometry influence on the slipstream. Quantitatively good results were reported, but a high level of information was required to accurately construct and panel the propeller geometry, making this tool less ideal for early design stages. 


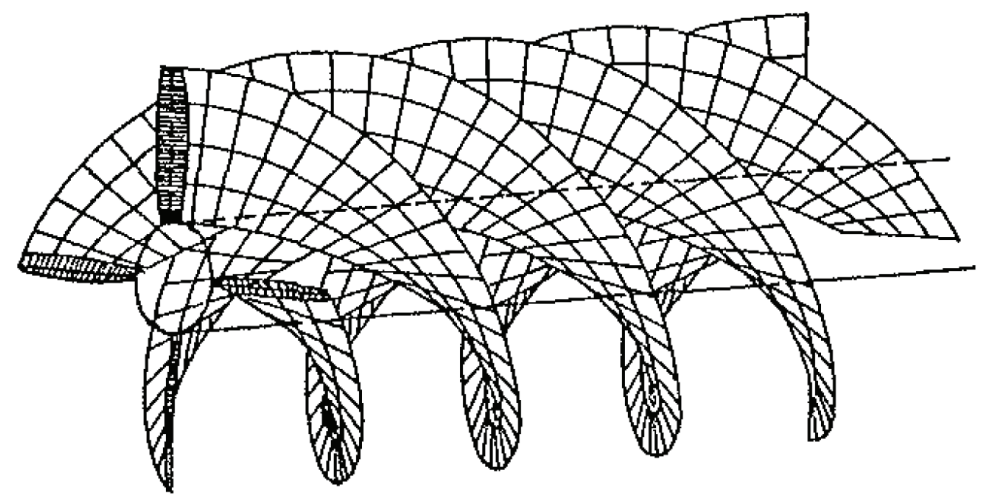

Figure 2.5: Helical trailing vortex panels from Yang, Li, and E. 8

\subsubsection{Frequency Domain Panel Method}

A unique approach to the propeller-airframe interaction problem was proposed by Cho and Williams. ${ }^{[27}$ They employed a frequency domain panel method, which broke down the periodic loading associated with a rotating propeller into harmonics, where the amplitudes were iteratively solved for. The overall method involved paneling both the propeller and the wing, with the solution depending on an inversion of a system of simultaneous equations. The method was validated for both a standalone propeller and a propeller wing combination, with good agreement in both cases. The value of the method lies in the ability to understand time dependent solutions, which were not captured in other past methods due to time averaging. The results were reported as similar to quasi-steady methods at low frequencies, indicating that the addition of periodic time modeling was not necessary in these cases. Additionally, Cho and Williams noted that a suitable model would have isolated time averaged propwash imposed on a wing for wing loading, as well as isolated time averaged wingwash 
imposed on the propeller to modify the propeller loading.

\subsubsection{Summary of Key Differences of Present Research}

The current research is different from each of the previously reviewed methods in a variety of ways that make it novel and interesting. Some aspects of the current research can be found in various past methods, but none of the methods combine all of the elements of this research. Additionally, some of the methods work well, but their application is different, because many of the methods incorporate full Euler solutions that dramatically increase computational time over what is desirable here.

None of the methods presented use vortex particles to examine the interaction effects between aerodynamic bodies and a propeller, which this research does. At the same time though, one method presented does use vortex particles to model the flowfield of a rotor, proving that the current application of vortex particles is feasible.

Only some of the methods make the simplification of using an actuator disk in place of a finite bladed propeller model. This assumption is vital to the philosophy of the proposed research because it prevents the necessity of knowing exact blade geometry and count to run cases, which is ideal for early stages of design. Additionally, it provides a pseudo-steady solution rather than the unsteady solution associated with a finite bladed propeller.

The choice of a panel code for calculation of aerodynamic solutions for this research is not unique when considering propeller flowfield interaction with various geometries, demonstrated by the fact that several of the previous methods employ panel codes. 
The panel code is the ideal choice for this research compared with a volume grid based method because of the target application. The desire is to create a tool that can be used in optimization cases early on in design, and the panel code allows for relative ease of meshing when compared to a CFD type solution, and a corresponding computational efficiency that accompanies the much lower grid sizing.

One of the major elements not captured in nearly all previous methods is the feedback from the geometry on the propeller flowfield. All of the methods aim to take into account how the propeller flowfield affects the geometry, but nearly all neglect the fact that the propeller flowfield will be deformed because of influence from the geometry. The lack of forced connectivity data between particles in general, as well as the fact that the propeller solution isn't completely steady, allow the flow to develop much more naturally around the geometry than was previously possible. 


\section{Chapter 3}

\section{Vortex Particle Theory}

In order to accurately model a propeller streamtube, rotational effects in a flow must be accounted for. Since by definition a flow is rotational if $\nabla \times \mathbf{V} \neq 0$ and $\boldsymbol{\omega} \equiv \nabla \times \mathbf{V}$ is the definition of vorticity, if vorticity is present the flow will be rotational. The presence of vorticity in rotational flows justifies the use of vortex particle schemes to model the effects of aircraft and rotor wakes. This capability of vortex particles led to their implementation as the rotational flow model in the present work. To best understand how vortex particles interact and evolve in the flowfield, it is first necessary to understand some fundamental particle theory.

\subsection{Fundamentals}

A vortex particle is an influencing element, the same way that the point source and point doublet elements are for a panel code. As such, each individual element exerts its own influence throughout the flowfield. Each vortex particle, also known as a vorton or vortex stick, has both a vector position and a vector strength associated with 
it, along with a volume and, if it is regularized, a vortex core radius. The particles can be thought of as discrete points along a vortex tube, with the advantage that there is no forced connectivity between particles, and no corresponding worries about intersecting vortex tubes. ${ }^{[28]}$ Additionally, the lack of connectivity allows for explicit treatment of viscous diffusion not otherwise possible. The main problem associated with the particle discretization is the loss of a guaranteed divergence free vorticity field. Vortex particle solutions are based around solving the inviscid momentum equation in vorticity-velocity form,, 3

$$
\frac{\partial \boldsymbol{\omega}}{\partial t}+(\nabla \cdot(\boldsymbol{\omega} \mathbf{u}))=(\boldsymbol{\omega} \cdot \nabla) \mathbf{u} .
$$

\subsection{Singular Particles}

The influencing effect of the particles is clear when examining the vorticity field produced by $p$ singular particles, which can be written as

$$
\boldsymbol{\omega}(\mathbf{x})=\sum_{p} \boldsymbol{\omega}^{p} v o l^{p} \delta\left(\mathbf{x}-\mathbf{x}^{p}\right)=\sum_{p} \boldsymbol{\alpha}^{p}(t) \delta\left(\mathbf{x}-\mathbf{x}^{p}(t)\right) .
$$

Thus, the vector vorticity, $\boldsymbol{\omega}$, at any vector location, $\mathbf{x}$, in the field is a function of the vorticity of each particle, the volume of each particle, and the Dirac delta function applied to the distance between the point in queston and the particle. The strength vector, $\boldsymbol{\alpha}$, is equal to the volume times the vorticity, $\boldsymbol{\alpha}=\boldsymbol{\omega}$ vol.

The streamfunction of the field can be obtained from the vorticity field through the relationship $\nabla^{2} \Psi(\mathbf{x}, t)=-\boldsymbol{\omega}(\mathbf{x}, t)$. According to Winckelmans, ${ }^{3]}$ the Green's 
function for $-\nabla^{2}$ in an unbounded three dimensional domain is $G(\mathbf{x})=1 /(4 \pi|\mathbf{x}|)$, which makes a solution for the streamfunction $\Psi$ possible, resulting in

$$
\Psi(\mathbf{x}, t)=G(\mathbf{x}) * \boldsymbol{\omega}(\mathbf{x}, t)=\sum_{p} G\left(\mathbf{x}-\mathbf{x}^{p}(t)\right) \boldsymbol{\alpha}^{p}(t)=\frac{1}{4 \pi} \sum_{p} \frac{\boldsymbol{\alpha}^{p}(t)}{\left|\mathbf{x}-\mathbf{x}^{p}(t)\right|}
$$

where $*$ denotes a convolution.

The vortex particle has a velocity influence that is singular at the particle, and decays as the distance from the particle increases. The velocity influence from a single particle can be derived by examining the vorticity field produced by that particle. The streamfunction is then found from the vorticity field, and the velocity field is simply the curl of the streamfunction,

$$
\mathbf{u}(\mathbf{x}, t)=\nabla \times \Psi(\mathbf{x}, t)=\sum_{p} \nabla\left(G\left(\mathbf{x}-\mathbf{x}^{p}(t)\right)\right) \times \boldsymbol{\alpha}^{p}(t)
$$

Evaluating Equation 3.4 by replacing $\Psi$ with its value from Equation 3.3 results in

$$
\mathbf{u}(\mathbf{x}, t)=-\frac{1}{4 \pi} \sum_{p} \frac{1}{\left|\mathbf{x}-\mathbf{x}^{p}(t)\right|^{3}}\left(\mathbf{x}-\mathbf{x}^{p}(t)\right) \times \boldsymbol{\alpha}^{p}(t)
$$

which can be written more simply as

$$
\mathbf{u}(\mathbf{x}, t)=\sum_{p} \mathbf{K}\left(\mathbf{x}-\mathbf{x}^{p}(t)\right) \times \boldsymbol{\alpha}^{p}(t)
$$

where $\mathbf{K} \equiv-\frac{1}{4 \pi} \frac{1}{\left|\mathbf{x}-\mathbf{x}^{p}(t)\right|^{3}}\left(\mathbf{x}-\mathbf{x}^{p}(t)\right) \times$ is the Biot-Savart kernel.

The major weakness of the vortex particle method is the fact that the vorticity field is not guaranteed to be divergence free for all times. The initial vorticity field can be adjusted to ensure that it is nearly divergence free, but as time evolves there 
is no inherent feature of the method that maintains this freedom from divergence. Although the vorticity field and streamfunction are not guaranteed to be divergence free, the velocity field is because it is the curl of a streamfunction. The divergence free vorticity field can be written as

$$
\boldsymbol{\omega}^{N}(\mathbf{x}, t)=\sum_{p}\left[\boldsymbol{\alpha}^{p}(t) \delta\left(\mathbf{x}-\mathbf{x}^{p}(t)\right)+\nabla\left(\boldsymbol{\alpha}^{p}(t) \cdot \nabla\left(\frac{1}{4 \pi\left|\mathbf{x}-\mathbf{x}^{p}(t)\right|}\right)\right)\right],
$$

which is rewritten by Winckelman $s^{3}$ after calculating the gradient terms as

$$
\begin{aligned}
\boldsymbol{\omega}^{N}(\mathbf{x}, t)= & \sum_{p}\left[\left(\delta\left(\mathbf{x}-\mathbf{x}^{p}(t)\right)-\frac{1}{4 \pi\left|\mathbf{x}-\mathbf{x}^{p}(t)\right|^{3}}\right) \boldsymbol{\alpha}^{p}(t)+\right. \\
& \left.3 \frac{\left(\left(\mathbf{x}-\mathbf{x}^{p}(t)\right) \cdot \boldsymbol{\alpha}^{p}(t)\right)}{4 \pi\left|\mathbf{x}-\mathbf{x}^{p}(t)\right|^{5}}\left(\mathbf{x}-\mathbf{x}^{p}(t)\right)\right] .
\end{aligned}
$$

The momentum equation as written in Equation 3.1 has several alternate forms $\frac{28[33}{\sqrt[23]{3}}$ These forms can be written

$$
\begin{gathered}
\frac{\partial \boldsymbol{\omega}}{\partial t}+\nabla \cdot(\boldsymbol{\omega} \mathbf{u})=(\boldsymbol{\omega} \cdot \nabla) \mathbf{u} \\
\frac{\partial \boldsymbol{\omega}}{\partial t}+\nabla \cdot(\boldsymbol{\omega} \mathbf{u})=\left(\boldsymbol{\omega} \cdot \nabla^{T}\right) \mathbf{u} \\
\frac{\partial \boldsymbol{\omega}}{\partial t}+\nabla \cdot(\boldsymbol{\omega} \mathbf{u})=\frac{1}{2}\left(\boldsymbol{\omega} \cdot\left(\nabla+\nabla^{T}\right)\right) \mathbf{u} .
\end{gathered}
$$

Equation 3.9 is known as the classical scheme, Equation 3.10 the transpose scheme, and Equation 3.11 the mixed scheme. The transpose scheme was selected for this research to remain consistent with the scheme implemented by Winckelmans. The three equations are only equal when the vorticity field is divergence free, and hence can cause different results over the course of numerical vortex particle simulations. Of the three, only the transpose scheme conserves total vorticity. 


\subsection{Regularized Particles}

While singular particles represent the basic theory, a brief examination of Equation 3.5 reveals a singularity that can cause significant problems in numerical simulations due to finite time steps. The finite time step of a numerical simulation can result in the instantaneously updated position of a particle being too close to a singularity, which would be prevented in reality by the continuous evolution of the particle position and increasing resistance from other particles. Regularized particles are therefore used in the vast majority of computational particle applications to avoid singularity issues.

Regularized particles incorporate a regularization function to remove the singularity by reducing influence to zero rather than increasing it toward infinity as radius is decreased. Figure 3.1 visually shows the difference between the singularity and regularization. Clearly, as the distance away from the particle increases, the kernel value for the singular and the regularized particles become equal. At minimal distances away from the particle however, the regularized kernel value returns to zero, with the slope changing sign at the core radius value.

The vorticity field is written slightly differently when the regularization function is applied.

$$
\boldsymbol{\omega}_{\sigma}(\mathbf{x}, t)=\zeta_{\sigma}(\mathbf{x}) * \boldsymbol{\omega}\left(\mathbf{x}-\mathbf{x}^{p}(t)\right)=\sum_{p} \boldsymbol{\alpha}^{p}(t) \zeta_{\sigma}\left(\mathbf{x}-\mathbf{x}^{p}(t)\right)
$$

where $\sigma$ is the vortex core radius and $\zeta_{\sigma}$ is a regularization function, of which there are infinite potential options. The option selected for this research is the high order 


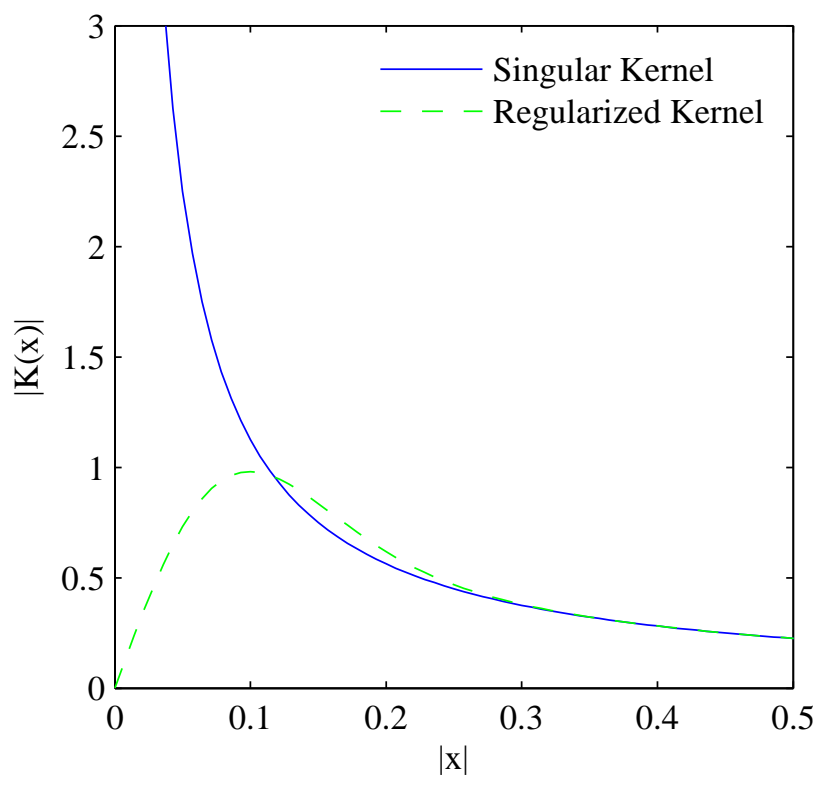

Figure 3.1: A comparison of a singular particle kernel with a regularized particle kernel for $\sigma=0.1$.

algebraic regularization scheme of Winckelmans, ${ }^{3}$ which is discussed in Section 3.5 . $\zeta_{\sigma}$ is defined as

$$
\zeta_{\sigma}(\mathbf{x}) \equiv \frac{1}{\sigma^{3}} \zeta\left(\frac{|\mathbf{x}|}{\sigma}\right)
$$

The regularized streamfunction can still be written as a function of the regularized vorticity field, similar to Equation 3.3 , as

$$
\nabla^{2} \Psi_{\sigma}(\mathbf{x}, t)=-\boldsymbol{\omega}_{\sigma}(\mathbf{x}, t)
$$

which means that with the use of the Green's function previously defined the streamfunction becomes

$$
\nabla^{2} \Psi_{\sigma}(\mathbf{x}, t)=G(\mathbf{x}) * \omega_{\sigma}(\mathbf{x}, t)
$$

To relate the streamfunction to the regularized vorticity field rather than the singular 
one, a $G_{\sigma}$ function is used in place of $G$ as follows,

$$
\nabla^{2} \Psi_{\sigma}(\mathbf{x}, t)=G_{\sigma}(\mathbf{x}) * \boldsymbol{\omega}(\mathbf{x}, t)=\sum_{p} G_{\sigma}\left(\mathbf{x}-\mathbf{x}^{p}(t)\right) \boldsymbol{\alpha}^{p}(t)
$$

where $G_{\sigma}(\mathbf{x})=G(|\mathbf{x}| / \sigma) / \sigma$ according to Winckelmans. ${ }^{3}$

The regularized velocity field is then obtained the same way as the singular velocity field was, through the curl of the streamfunction.

$$
\mathbf{u}_{\sigma}(\mathbf{x}, t)=\nabla \times \Psi_{\sigma}(\mathbf{x}, t)=\sum_{p} \nabla\left(G_{\sigma}\left(\mathbf{x}-\mathbf{x}^{p}(t)\right)\right) \times \boldsymbol{\alpha}^{p}(t)
$$

which can be rewritten in numerical form as

$$
\mathbf{u}_{\sigma}(\mathbf{x}, t)=-\sum_{p} \frac{q_{\sigma}\left(\mathbf{x}-\mathbf{x}^{p}(t)\right)}{\left|\mathbf{x}-\mathbf{x}^{p}(t)\right|^{3}}\left(\mathbf{x}-\mathbf{x}^{p}(t)\right) \times \boldsymbol{\alpha}^{p}(t)
$$

where $-\left(q_{\sigma}(\mathbf{x}) /|\mathbf{x}|^{3}\right) \mathbf{x} \times$ is a regularized Biot-Savart kernel. $\stackrel{3}{3}$

Just like the singular particles, there are multiple formulations for the momentum equation. The classical scheme is

$$
\frac{\partial \boldsymbol{\omega}}{\partial t}+\nabla \cdot(\boldsymbol{\omega} \mathbf{u})=(\boldsymbol{\omega} \cdot \nabla) \mathbf{u}_{\sigma}
$$

while the transpose scheme is

$$
=\left(\boldsymbol{\omega} \cdot \nabla^{T}\right) \mathbf{u}_{\sigma}
$$

and the mixed scheme is

$$
=\frac{1}{2}\left(\boldsymbol{\omega} \cdot\left(\nabla+\nabla^{T}\right)\right) \mathbf{u}_{\sigma}
$$

As with singular particles, the regularized particles suffer the from the same lack of guarantee of a divergence free field. For the regularized particles a divergence free 
vorticity field can be written as

$$
\boldsymbol{\omega}_{\sigma}^{N}(\mathbf{x}, t)=\sum_{p}\left[\boldsymbol{\alpha}^{p}(t) \zeta_{\sigma}\left(\mathbf{x}-\mathbf{x}^{p}(t)\right)+\nabla\left(\boldsymbol{\alpha}^{p}(t) \cdot \nabla\left(G_{\sigma}\left(\mathbf{x}-\mathbf{x}^{p}(t)\right)\right)\right)\right]
$$

Winckelmans rewrites Equation 3.22 as

$$
\begin{gathered}
\boldsymbol{\omega}_{\sigma}^{N}(\mathbf{x}, t)=\sum_{p}\left[\left(\zeta_{\sigma}\left(\mathbf{x}-\mathbf{x}^{p}(t)\right)-\frac{q_{\sigma}\left(\mathbf{x}-\mathbf{x}^{p}(t)\right)}{\left|\mathbf{x}-\mathbf{x}^{p}(t)\right|^{3}}\right) \boldsymbol{\alpha}^{p}(t)+\right. \\
\left.\left(3 \frac{q_{\sigma}\left(\mathbf{x}-\mathbf{x}^{p}(t)\right)}{\left|\mathbf{x}-\mathbf{x}^{p}(t)\right|^{3}}-\zeta_{\sigma}\left(\mathbf{x}-\mathbf{x}^{p}(t)\right)\right) \frac{\left(\left(\mathbf{x}-\mathbf{x}^{p}(t)\right) \cdot \boldsymbol{\alpha}^{p}(t)\right)}{\left|\mathbf{x}-\mathbf{x}^{p}(t)\right|^{2}}\left(\mathbf{x}-\mathbf{x}^{p}(t)\right)\right] .
\end{gathered}
$$

It has been shown that the regularized particle method converges to the solution of the respective formulation of the momentum equation selected, either classical, transpose, or mixed, for some finite time T.29] $31[33$ The proofs show that as the number of particles increases and the corresponding vortex core sizes decrease, the error norm decreases to zero.

\subsection{Viscous Diffusion}

As mentioned, one of the major strengths of the vortex particle method is its ability to account for viscous diffusion. This feature is extremely valuable because it has been demonstrated to help maintain a nearly divergence-free particle vorticity

field necessary for a valid solution. ${ }^{34}$ The transpose scheme applied to the vorticityvelocity form of the momentum equation with viscous effects is written

$$
\frac{\partial \boldsymbol{\omega}}{\partial t}+\nabla \cdot(\boldsymbol{\omega} \mathbf{u})=\left(\boldsymbol{\omega} \cdot \nabla^{T}\right) \mathbf{u}+\nu \nabla^{2} \boldsymbol{\omega}
$$


where the additional term at the end of the right hand side accounts for diffusion. It is important to note that the viscous term $\nu \nabla^{2} \boldsymbol{\omega}$ is independent of the form of the momentum equation, either classical, transpose, or mixed.

The diffusion is modeled by adjusting particle strengths $\boldsymbol{\alpha}^{p}(t)$ to reflect viscous diffusion. $\sqrt[35]{37}$ The method approximates the Laplacian appearing in the viscous term with an integral, which is further discretized by regularized particles. Considering a radially symmetric regularization function that satisfies

$$
\int_{0}^{\infty}\left|\zeta^{\prime}(\rho)\right| \rho^{3+r} d \rho<\infty
$$

define a function of the regularized particles

$$
\eta_{\sigma}(\mathbf{x})=\frac{1}{\sigma^{5}} \eta\left(\frac{|\mathbf{x}|}{\sigma}\right)
$$

where

$$
\eta(\rho)=-\frac{\zeta^{\prime}(\rho)}{\rho}
$$

For this case, the Laplacian of some function, $f(\mathbf{x})$ can be approximated by

$$
\nabla^{2} f(\mathbf{x}) \simeq 2 \int(f(\mathbf{y})-f(\mathbf{x})) \eta_{\sigma}(\mathbf{x}-\mathbf{y}) d \mathbf{y}
$$

Winckelmans notes that the low order algebraic smoothing does not satisfy Equation 3.25, and therefore is not a good choice to model diffusion with.

Winckelman also shows that considering the convection-diffusion equation

$$
\frac{\partial f}{\partial t}+\nabla \cdot(f \mathbf{u})=\nu \nabla^{2} f
$$


it can be approximated through the proposed integral as

$$
\frac{\partial f}{\partial t}+\nabla \cdot(f \mathbf{u})=2 \nu \int(f(\mathbf{y})-f(\mathbf{x})) \eta_{\sigma}(\mathbf{x}-\mathbf{y}) d \mathbf{y}
$$

Considering a particle approximation to $f(\mathbf{x}, t)$, written as

$$
f_{\sigma}(\mathbf{x}, t)=\sum_{p}\left(f^{p}(t) v o l^{p}(t)\right) \zeta_{\sigma}\left(\mathbf{x}-\mathbf{x}^{p}(t)\right)
$$

allows for a particle solution to the convection-diffusion problem, leading to

$$
\begin{gathered}
\frac{d}{d t} \mathbf{x}^{p}(t)=\mathbf{u}\left(\mathbf{x}^{p}(t), t\right) \\
\frac{d}{d t} v^{p l}(t)=v o l^{p}(t) \nabla \cdot \mathbf{u}\left(\mathbf{x}^{p}(t), t\right) \\
\frac{d}{d t}\left(f^{p}(t) \operatorname{vol}^{p}(t)\right)=2 \nu \operatorname{vol}^{p}(t) \sum_{q} v o l^{q}(t)\left(f^{q}(t)-f^{p}(t)\right) \eta_{\sigma}\left(\mathbf{x}^{p}(t)-\mathbf{x}^{q}(t)\right) .
\end{gathered}
$$

According to Winckelmans this double approximation results in a error in the diffusion term of $\mathscr{O}\left(\nu\left(\sigma^{r}+h^{m} / \sigma^{m+1}\right)\right)$. For high viscosity values the viscous diffusion error is higher than that associated with the particle approximation of the convection term, but for low $\nu$ the error is lower than for the convective term. These errors assume that $\eta(\rho)>0$ which requires regularization functions of second order, such as the high order algebraic regularization. It is also possible to extend this method to a nonconstant value of $\nu$ in the flowfield, although that was not investigated for this work. ${ }^{36}$ It is important to note that the particle discretization of the integral approximation of the Laplacian is conservative. As such, the total particle strengths don't decay over time, but rather are redistributed in a manner reflective of diffusion. 
Solving Equation 3.24 yields

$$
\begin{gathered}
\frac{d}{d t} \mathbf{x}^{p}(t)=\mathbf{u}_{\sigma}\left(\mathbf{x}^{p}(t), t\right) \\
\frac{d}{d t} \boldsymbol{\alpha}^{p}(t)=\left(\boldsymbol{\alpha}^{p}(t) \cdot \nabla^{T}\right) \mathbf{u}_{\sigma}\left(\mathbf{x}^{p}(t), t\right)+ \\
2 \nu \sum_{q}\left(\operatorname{vol}^{p} \boldsymbol{\alpha}^{q}(t)-\operatorname{vol}^{q} \boldsymbol{\alpha}^{p}(t)\right) \eta_{\sigma}\left(\mathbf{x}^{p}(t)-\mathbf{x}^{q}(t)\right) .
\end{gathered}
$$

When considering the high order algebraic regularization,

$$
\zeta^{\prime}(\rho)=\frac{d}{d \rho}\left(\frac{15}{8 \pi\left(\rho^{2}+1\right)^{7 / 2}}\right)=\frac{-105 \rho}{8 \pi\left(\rho^{2}+1\right)^{9 / 2}}
$$

so

$$
\eta(\rho)=-\frac{\left(\frac{-105 \rho}{8 \pi\left(\rho^{2}+1\right)^{9 / 2}}\right)}{\rho}=\frac{105}{8 \pi\left(\rho^{2}+1\right)^{9 / 2}},
$$

and $\eta_{\sigma}(\rho)=\frac{1}{\sigma^{5}} \eta(\rho)$ so $\eta_{\sigma}$ becomes

$$
\eta_{\sigma}(\rho)=\frac{1}{\sigma^{5}} \frac{105}{8 \pi\left(\rho^{2}+1\right)^{9 / 2}}
$$

\subsection{Evolution Equations}

As previously mentioned, singular particles are simply not well suited to computational flow simulations, and regularization is required whenever numerically based simulations are conducted. As such, the computational forms of the evolution equations are presented here for the regularized particle scheme using the high order algebraic regularization functions. These forms of the equations much more directly show how the theory is implemented in MATLAB. 
The generic evolution equations used in the vortex particle scheme implemented here are

$$
\frac{d}{d t} \mathbf{x}^{p}(t)=\mathbf{u}_{\sigma}\left(\mathbf{x}^{p}(t), t\right)
$$

for the position update of particle $p$ and

$$
\frac{d}{d t} \boldsymbol{\alpha}^{p}(t)=\left(\boldsymbol{\alpha}^{p}(t) \cdot \nabla^{T}\right) \mathbf{u}_{\sigma}\left(\mathbf{x}^{p}(t), t\right)
$$

for the strength update. The strength update equation is also referred to as the stretching equation or vortex stretching equation. Equation 3.39 is the transpose formulation of the strength evolution equation, which was selected to remain consistent with the work of Winckelmans. It has been proven by various sources that the grid free vortex scheme described by Equations 3.38 and 3.39 converges to the Euler equations 3133 The basic conclusion of the convergence studies is that, as the number of particles increases, and the vortex core size correspondingly decreases, the error associated with the vortex particle discretization of the vorticity field is reduced to zero.

For Winckelmans' high order algebraic regularization the following definitions apply

$$
\begin{gathered}
\zeta(\rho)=\frac{7.5}{4 \pi\left(\rho^{2}+1\right)^{7 / 2}}, \\
G(\rho)=\frac{\rho^{2}+1.5}{\left(\rho^{2}+1\right)^{3 / 2}},
\end{gathered}
$$

and

$$
q(\rho)=\frac{\rho^{3}}{4 \pi\left(\rho^{2}+1\right)^{3 / 2}} .
$$


The high order algebraic regularization function benefits from having similar convergence properties to the more commonly used Gaussian regularization functions, but with much simpler $G$ and $q$ functions. Also, the gradients of the velocity are relatively easy to analytically calculate, making $\frac{\partial \alpha}{\partial t}$ more accessible. Additionally, it is the only known regularization for which analytical equations for total vorticity, linear impulse, angular impulse, kinetic energy, and enstrophy can be derived. The analytical relationships are a feature missing for any other three dimensional regularization function.

Applying the high order algebraic regularization functions to the evolution equations results in their final numerical forms. The position update is written as

$$
\frac{d}{d t} \mathbf{x}^{p}=-\frac{1}{4 \pi} \sum_{q} \frac{\left|\mathbf{x}^{p}-\mathbf{x}^{q}\right|^{2}+\frac{5}{2} \sigma^{2}}{\left(\left|\mathbf{x}^{p}-\mathbf{x}^{q}\right|^{2}+\sigma^{2}\right)^{5 / 2}}\left(\mathbf{x}^{p}-\mathbf{x}^{q}\right) \times \boldsymbol{\alpha}^{q},
$$

while the strength update with viscous diffusion is written

$$
\begin{gathered}
\frac{d}{d t} \boldsymbol{\alpha}^{p}=\frac{1}{4 \pi} \sum_{q}\left[\frac{\left|\mathbf{x}^{p}-\mathbf{x}^{q}\right|^{2}+\frac{5}{2} \sigma^{2}}{\left(\left|\mathbf{x}^{p}-\mathbf{x}^{q}\right|+\sigma^{2}\right)^{5 / 2}} \boldsymbol{\alpha}^{p} \times \boldsymbol{\alpha}^{q}+\right. \\
+3 \frac{\left|\mathbf{x}^{p}-\mathbf{x}^{q}\right|^{2}+\frac{7}{2} \sigma^{2}}{\left(\left|\mathbf{x}^{p}-\mathbf{x}^{q}\right|^{2}+\sigma^{2}\right)^{7 / 2}}\left(\boldsymbol{\alpha}^{p} \cdot\left(\left(\mathbf{x}^{p}-\mathbf{x}^{q}\right) \times \boldsymbol{\alpha}^{q}\right)\right)\left(\mathbf{x}^{p}-\mathbf{x}^{q}\right)+ \\
\left.105 \nu \frac{\sigma^{4}}{\left(\left|\mathbf{x}^{p}-\mathbf{x}^{q}\right|^{2}+\sigma^{2}\right)^{9 / 2}}\left(v o l^{p} \boldsymbol{\alpha}^{q}-v o l^{q} \boldsymbol{\alpha}^{p}\right)\right] .
\end{gathered}
$$

\subsection{Diagnostics}

One of the fundamental components of verifying that the vortex particle scheme is implemented correctly is defining a measurement tool to use for comparison $\sqrt[3]{34} \sqrt[38]{38}$ 
The original numerical simulation uses several diagnostic terms, which have be recalculated for comparison. Those diagnostic terms fall into two categories; linear and quadratic. The linear diagnostics are total vorticity, linear impulse, and angular impulse, while the quadratic diagnostics are enstrophy, helicity, and kinetic energy. Winckelmans provides complete derivations of each of the diagnostic terms. ${ }^{3}$ The diagnostics, much like particle velocity and stretching, are dependent on the regularization function chosen. As such, all diagnostics examined here will be for the high order algebraic regularization function.

The diagnostics provide a valuable tool during numerical simulation because they can assess the level of numerical error. The linear diagnostics should all be conserved when the particle vorticity field remains a good approximation of the true vorticity field. The transpose scheme in particular should conserve the total vorticity, and that is the scheme utilized here. Additionally, the linear impulse should be conserved as long as the particle vorticity field remains approximately divergence free, and so tracking its evolution over time indicates the relative state of the particle field. The quadratic diagnostics kinetic energy and enstrophy each have two formulations, one of which is for the theoretical divergence free field, and one is for the regularized particle approximation. Comparing the two forms of each diagnostic over time provides additional insight into whether or not the particle field is remaining nearly divergence free. 


\subsubsection{Linear Diagnostics}

The linear diagnostics are much more simple than their quadratic counterparts. They can easily be expressed for the high order algebraic function, and are less computationally expensive to calculate. The first of the linear diagnostics, total vorticity, can be calculated as

$$
\boldsymbol{\Omega}=\sum_{p} \boldsymbol{\alpha}^{p}(t),
$$

so the total vorticity is simply the sum of all $p$ of the vortex particle strengths in the field at time $t$. The linear impulse is defined as

$$
\mathbf{I}=\frac{1}{2} \sum_{p} \mathbf{x}^{p}(t) \times \boldsymbol{\alpha}^{p}(t) .
$$

The angular impulse is defined as

$$
\mathbf{A}=\frac{1}{2} \sum_{p} \mathbf{x}^{p}(t) \times\left(\mathbf{x}^{p}(t) \times \boldsymbol{\alpha}^{p}(t)\right)-\frac{1}{3} C \sigma^{2} \boldsymbol{\Omega},
$$

where

$$
C=4 \pi \int_{0}^{\infty} \zeta(\rho) \rho^{4} d \rho .
$$

The second term in Equation 3.47 obviously becomes zero when the total vorticity is zero. The transpose formulation of the stretching function conserves total vorticity, and the initial vorticity at $t=0$ is $\Omega=0$, so the angular impulse can be reduced to

$$
\mathbf{A}=\frac{1}{2} \sum_{p} \mathbf{x}^{p}(t) \times\left(\mathbf{x}^{p}(t) \times \boldsymbol{\alpha}^{p}(t)\right) .
$$




\subsubsection{Quadratic Diagnostics}

The quadratic diagnostics are more complex than the linear terms, and are consequently more difficult to calculate and to derive. The difficulty stems from the fact that it is challenging to analytically integrate terms such as

$$
E=\frac{1}{2} \int \mathbf{u}_{\sigma} \cdot \mathbf{u}_{\sigma} d x .
$$

Instead, the simplifying assumption of only using one regularized velocity term combined with one singular term, $\mathbf{u} \cdot \mathbf{u}_{\sigma}$, makes it possible, although only for certain

regularization functions, to complete the integrals $\sqrt[34]{34}$ One of the strengths of the high order algebraic regularization function is that analytical integration is possible for all the quadratic diagnostic terms, and Winckelmans believes it to be the only regularization function for which is this possible. It is important to note that the simplification of using only a single regularization term rather than both terms has varying levels of validity depending on which quadratic diagnostic is being evaluated. The assumption is most appropriate for kinetic energy, moderately valid for helicity, and poor for enstrophy ${ }^{3}$

There are two forms of both the kinetic energy and enstrophy quadratic diagnostics, one of which is technically invalid because it does not account for the non-zero divergence of the streamfunction that appears with the use of vortex particles. The comparison of the two terms for each diagnostic provides insight into how well the divergence free field is being preserved over time. This check indicates the numer- 
ical error associated with the simulation, because the vorticity field divergence is introduced through numerical approximations. Winckelmans shows that incorrectly assuming a divergence free field for kinetic energy yields

$$
\tilde{E}_{f}=\frac{1}{8 \pi} \sum_{p, q} \frac{\left(\left|\mathbf{x}^{p}-\mathbf{x}^{q}\right|^{2}+\frac{3}{2} \sigma^{2}\right)}{\left(\left|\mathbf{x}^{p}-\mathbf{x}^{q}\right|^{2}+\sigma^{2}\right)^{3 / 2}} \boldsymbol{\alpha}^{p} \cdot \boldsymbol{\alpha}^{q},
$$

while the correct equation for kinetic energy of a field of vortex particles is

$$
\begin{gathered}
\tilde{E}=\frac{1}{16 \pi} \sum_{p, q}\left[2 \frac{\rho}{\left(\rho^{2}+1\right)^{1 / 2}} \boldsymbol{\alpha}^{p} \cdot \boldsymbol{\alpha}^{q}+\right. \\
\left.\frac{\rho^{3}}{\left(\rho^{2}+1\right)^{3 / 2}}\left(\frac{\left(\left(\mathbf{x}^{p}-\mathbf{x}^{q}\right) \cdot \boldsymbol{\alpha}^{p}\right)}{\left|\mathbf{x}^{p}-\mathbf{x}^{q}\right|} \frac{\left.\left(\mathbf{x}^{p}-\mathbf{x}^{q}\right) \cdot \boldsymbol{\alpha}^{q}\right)}{\left|\mathbf{x}^{p}-\mathbf{x}^{q}\right|}-\boldsymbol{\alpha}^{p} \cdot \boldsymbol{\alpha}^{q}\right)\right] .
\end{gathered}
$$

Incorrectly assuming a divergence free field for enstrophy yields

$$
\tilde{\varepsilon}_{f}=\frac{1}{4 \pi} \sum_{p, q} \frac{15}{2} \frac{\sigma^{4}}{\left(\left|\mathbf{x}^{p}-\mathbf{x}^{q}\right|^{2}+\sigma^{2}\right)^{7 / 2}} \boldsymbol{\alpha}^{p} \cdot \boldsymbol{\alpha}^{q} .
$$

A relationship exists that correctly accounts for the divergence introduced by the particle approximation, however it appears that an error was present in equation given by Winckelmans. The equation is reproduced below, with the warning that an error may be present.

$$
\begin{gathered}
\tilde{\varepsilon}=\frac{1}{4 \pi} \sum_{p, q} \frac{1}{\sigma^{3}}\left[\frac{\left(5-\rho^{2}\left(\rho^{2}+\frac{7}{2}\right)\right)}{\left(\rho^{2}+1\right)^{7 / 2}} \boldsymbol{\alpha}^{p} \cdot \boldsymbol{\alpha}^{q}+\right. \\
\left.3 \frac{\left(\rho^{2}\left(\rho^{2}+\frac{9}{2}\right)+\frac{7}{2}\right)}{\left(\rho^{2}+1\right)^{9 / 2}}\left(\left(\left(\mathbf{x}^{p}-\mathbf{x}^{q}\right) \cdot \boldsymbol{\alpha}^{p}\right)\right)\left(\left(\left(\mathbf{x}^{p}-\mathbf{x}^{q}\right) \cdot \boldsymbol{\alpha}^{q}\right)\right)\right] .
\end{gathered}
$$

The helicity is less useful because it does not have multiple forms for numerical comparison. It can be written as

$$
\mathscr{H}=\frac{1}{4 \pi} \sum_{p, q} \frac{\left(\left|\mathbf{x}^{p}-\mathbf{x}^{q}\right|^{2}+\frac{5}{2} \sigma^{2}\right)}{\left(\left|\mathbf{x}^{p}-\mathbf{x}^{q}\right|^{2}+\sigma^{2}\right)^{5 / 2}}\left(\left(\mathbf{x}^{p}-\mathbf{x}^{q}\right) \cdot\left(\boldsymbol{\alpha}^{p} \times \boldsymbol{\alpha}^{q}\right)\right) .
$$




\section{Chapter 4}

\section{Particle Scheme Verification}

With an understanding of vortex particle theory firmly in grasp, the next step was to create MATLAB code capable of applying that theory. In order to ensure that the particle method was implemented correctly, the single vortex ring simulation conducted by Winckelmans was recreated ${ }^{[3}$ The simulation was quite involved, but the result was a set of vortex particle evolution equations, as well as a useful discretization method and a powerful time integration scheme, all of which would be put to good use over the course of the project.

\subsection{Particle Discretization}

A key starting point for matching the vortex particle simulation of Winckelmans was to match the method of placing particles in the field. The simulation was of a single vortex ring with radius $R=1$ decaying over 5 simulated seconds. Particles were placed in disks, with the ring composed of a specified number of the disks. The particles were placed in such a manner that they each had equal area in their two 
dimensional disk. The first step was to place a single particle at the center of the disk. The method allowed for selection of a desired number of layers per disk, which would alter the number of particles present. Each layer is composed of a number of circumferentially distributed cells of equal area. A total of $N_{\varphi}$ disks are used to create the ring.

If the number of additional layers, $n_{c}$, is zero then no additional particles are used in the disk beyond the center particle. If, however, $n_{c} \neq 0$ then $n_{c}$ additional layers are added. Each additional layer has 8 more particles than the previous layer did, so that the final layer has $8 n_{c}$ particles in it. Each particle is assigned to what will be referred to as a cell. The cell for the particle placed at the center of the disk has a radius of $r_{l}$, and the cell for each additional particle is $2 r_{l}$ high in the radial direction. The radial boundaries for cells beyond the center cell are $r_{1}=(2 n-1) r_{l}$ and $r_{2}=(2 n+1) r_{l}$, where $n$ is the current layer being examined, between 1 and $n_{c}$. The particles are placed at the area centroid of each cell, whose radial position can be calculated as $r_{c}=\left(\left(1+12 n^{2}\right) / 6 n\right) r_{l}$. The maximum radius reached through the discretization is $r_{\max }=\left(2 n_{c}+1\right) r_{l}$. The total number of particles in a single disk composed of $n_{c}$ layers is $N_{s}=1+4 n_{c}\left(n_{c}+1\right)$. This scheme would later prove useful for discretizing an actuator disk, and a sample of the particle discretization can be seen in Figure 4.1. Much like $r_{1}$ and $r_{2}$ are the bounding radial components of a particle cell, $\theta_{1}$ and $\theta_{2}$ define the angles associated with the side boundaries of a cell, and each of these are also shown in Figure 4.1 . 


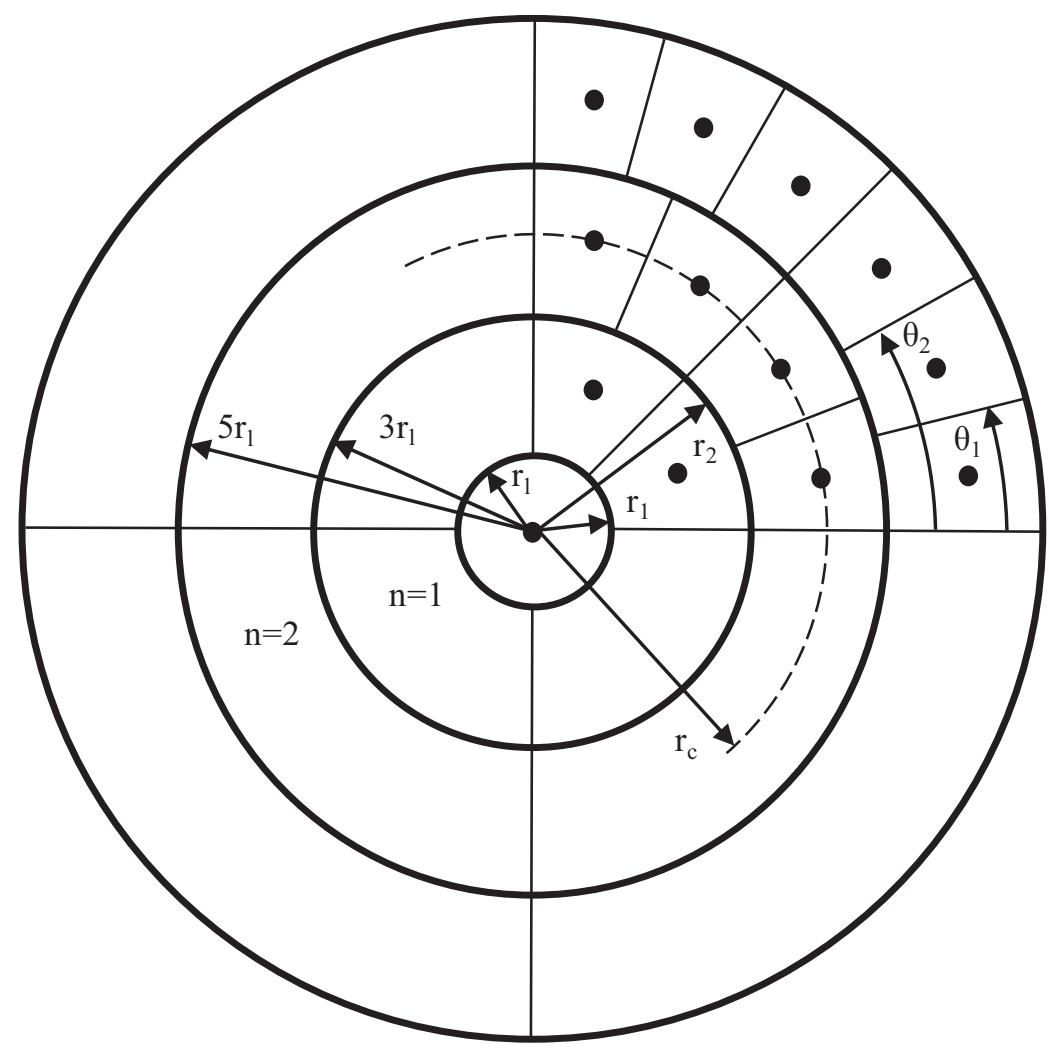

Figure 4.1: Discretization of layers of particles into a two dimensional disk with various discretization variables displayed ${ }^{3}$

Each cell has an area of $A=\pi r_{l}^{2}$, which is demonstrated in Appendix A, however since the problem occurs in three dimensions it is necessary for the particles to have a volume rather than an area. The circumferential angle between disks that compose the ring can be found as $\Delta \varphi=2 \pi / N_{\varphi}$. With all these definitions the cell volume is defined as

$$
\text { vol }=\Delta \varphi\left(r_{2}-r_{1}\right)\left[\left(\theta_{2}-\theta_{1}\right) R\left(\frac{r_{1}+r_{2}}{2}\right)+\left(\sin \left(\theta_{2}\right)-\sin \left(\theta_{1}\right)\right)\left(\frac{r_{1}^{2}+r_{1} r_{2}+r_{2}^{2}}{3}\right)\right]
$$

The complete vortex ring discretized by 6,480 particles is shown in Figure 4.2 . 


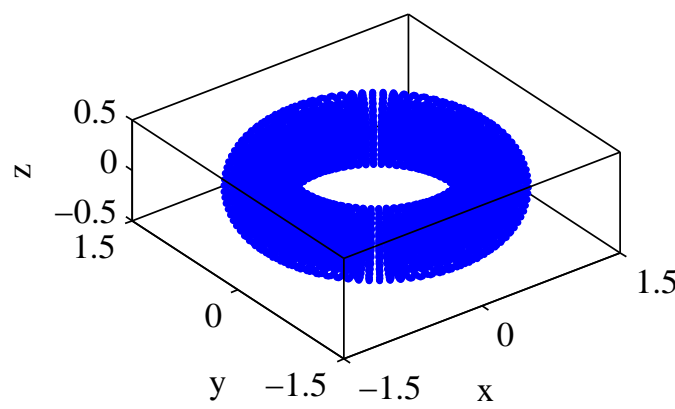

(a) Isometric

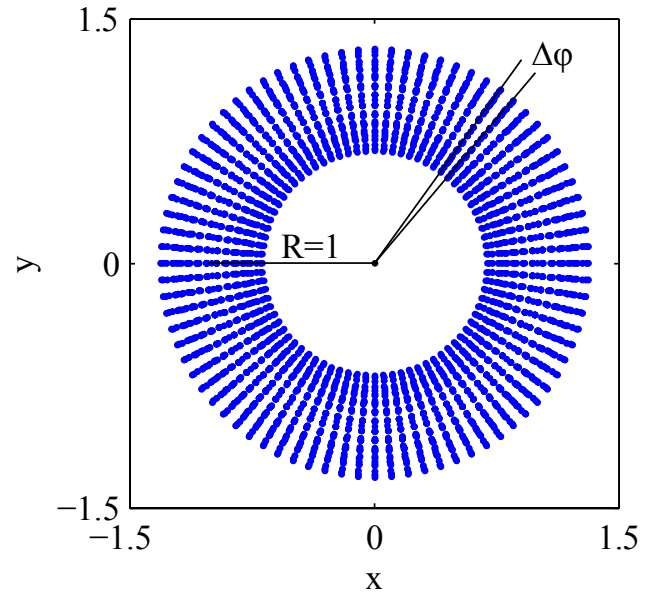

(b) Top

Figure 4.2: Vortex ring modeled by 6,480 particles from an isometric view as well as from a top view, with the top view showing the spacing between each particle disk.

\subsubsection{Initial Strength Assignment}

For this particular study $n_{c}=4$ was selected because of memory constraints related to creating an $N$ by $N$ matrix. An $n_{c}=4$ led to $N_{s}=81$ particles per disk, with $N_{\varphi}=80$ disks to construct the ring, for a total of $N=6,480$ particles in the entire study. One important feature of the single vortex ring is that the geometry is completely axisymmetric. A consequence of this fact is that influences only needed to be calculated on a single disk from all of the particles in the field, and then those influences were applied to all the disks in the field, which dramatically reduced computational expense, but not memory requirements.

The vortex core radius was set to ensure equal spacing between particles both in disk and between disks around the ring. The initial spacing is taken as the radial distance between particles. The number of disks composing a ring was then set at 
$N_{\varphi}=80$ so that the between-disk spacing was equal to the in-disk spacing.

Once the particles were discretized in the field the next step was to assign the strength vectors to those particles. The overall vortex ring was given a circulation of $\Gamma=1 \frac{f t^{2}}{s}$. The Reynolds number was set to 400 , and the corresponding viscosity value was $\nu=\Gamma / R e=2.50 \times 10^{-3} \frac{f t^{2}}{s}$. The initial vorticity field was then discretized using

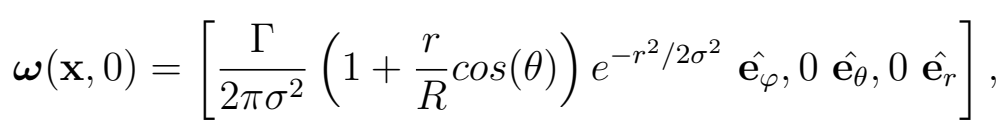

where $\sigma$ is the vortex core size of the particles related to the regularization function. Winckelmans states that the term in parenthesis is a correction which accounts for vorticity stretching. The unit vector, $\hat{\mathbf{e}}_{\varphi}$, indicates that the vorticity vector for each particle was always completely in the $\varphi$ axis, meaning that the vector was always tangent to the vortex ring, or alternatively the vorticity vectors were always orthogonal to the disk they are in. The particle strength vector $\boldsymbol{\alpha}$, also known as the particle strength, was then assigned as a function of the vorticity vector through

$$
\boldsymbol{\alpha}^{p}(0)=v o l^{p} \boldsymbol{\omega}\left(\mathbf{x}^{p}(0), 0\right) .
$$

The strength vectors for the particles making up a single disk are shown in vector form in Figure 4.3. Once the particle strength vectors had been assigned, the initial enstrophy was calculated and used to ensure that kinetic energy was decaying correctly. The viscous computation should decay as $\frac{d E}{d t}=-\nu \varepsilon$, and the initial enstrophy 


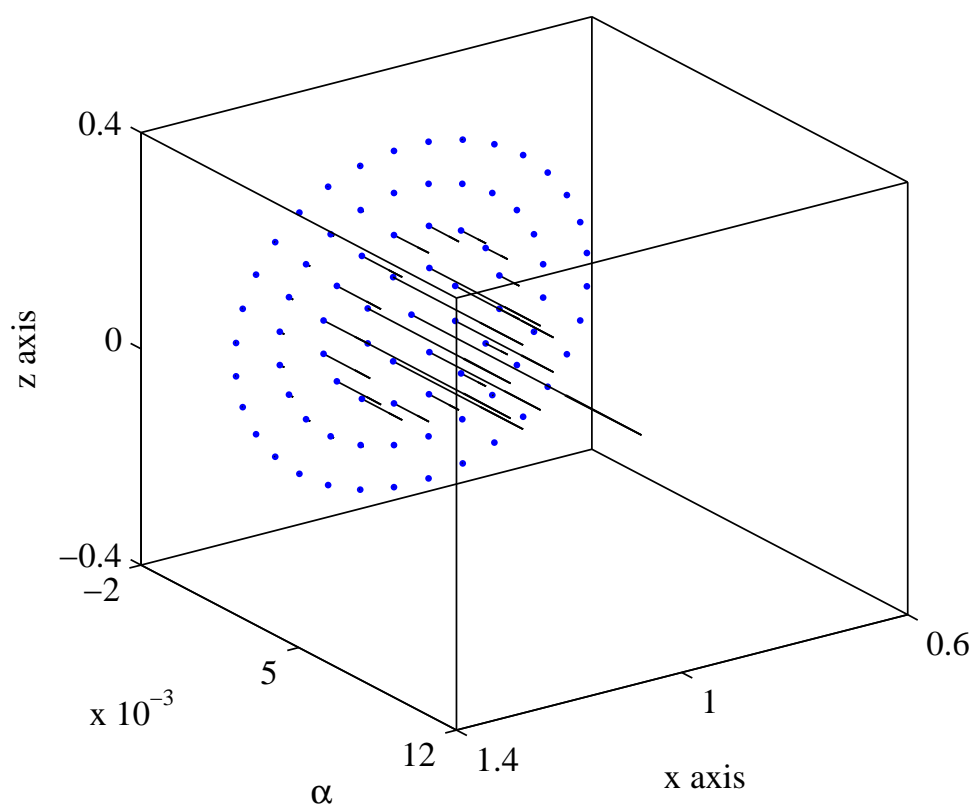

Figure 4.3: Vortex particle strength vectors for all particles in a single disk of the vortex ring.

can be calculated as

$$
\varepsilon(0)=\frac{\Gamma^{2} R}{2 \sigma^{2}}\left(1+\frac{3}{2} \frac{\sigma^{2}}{R^{2}}\right) .
$$

\subsection{Beale's Method}

As mentioned in the discussion of the diagnostic terms, the particle vorticity field wasn't guaranteed to be divergence free, unlike the theoretical vorticity field. To correct this, a technique called Beale's Method, or relaxation of the initial condition, was implemented, and the field was altered to reduce the divergence as much as possible $\sqrt[3]{39}$ The non divergence free vortex particle strength vectors were assigned through Equation 4.3. These strength vectors were adjusted through Beale's Method so that the particle vorticity field $\boldsymbol{\omega}_{\sigma}\left(\mathbf{x}^{p}(0), 0\right)$ was equal to the divergence 
free vorticity field, $\boldsymbol{\omega}\left(\mathbf{x}^{p}(0), 0\right)$.

Mathematically, new particle strength vectors are desired that cause the particle vorticity field and exact vorticity to be equal,

$$
\boldsymbol{\omega}\left(\mathbf{x}^{p}(0), 0\right)=\boldsymbol{\omega}_{\sigma}\left(\mathbf{x}^{p}(0), 0\right) .
$$

The particle discretized vorticity field of the left hand side, for which the particle strength vector is the unknown, is defined in Equation 3.12. The exact vorticity field is defined as $\boldsymbol{\omega}\left(\mathbf{x}^{p}(0), 0\right)=\boldsymbol{\alpha}^{p}(0) /$ vol $^{p}$. Plugging these definitions into Equation 4.5 gives

$$
\sum_{q} \boldsymbol{\alpha}_{\text {new }}^{q}(0) \zeta_{\sigma}\left(\mathbf{x}^{p}-\mathbf{x}^{q}(0)\right)=\frac{\boldsymbol{\alpha}_{\text {old }}^{p}(0)}{v_{o l}^{p}} .
$$

Thus, the new particle strength vectors, $\boldsymbol{\alpha}_{n e w}$, were a function of the old strength vectors, $\boldsymbol{\alpha}_{\text {old }}$, the particle locations, $\mathbf{x}_{p}$ and $\mathbf{x}_{q}$, and the particle volumes, vol, as well as the regularization function, $\zeta_{\sigma}$. By defining the matrix $[A]=\left[\operatorname{vol}_{p} \zeta_{\sigma}\left(\mathbf{x}_{p}-\mathbf{x}_{q}\right)\right]$ Equation 4.6 can be rewritten as

$$
[A] \boldsymbol{\alpha}_{n e w}=\boldsymbol{\alpha}_{\text {old }}
$$

For high particle counts in the study it quickly becomes impossible to invert the $[A]$ matrix, and to circumvent this problem an iterative scheme has been employed, relying on the fact that the initial strengths, $\boldsymbol{\alpha}_{\text {old }}$, are good guesses for the final strength values, $\boldsymbol{\alpha}_{n e w}$, that make the vortex particle vorticity field divergence free.

The iterative scheme suggested by Beale ${ }^{40}$ recasts Equation 4.6 as

$$
(A-I) \boldsymbol{\alpha}_{n e w}+\boldsymbol{\alpha}_{n e w}=\boldsymbol{\alpha}_{o l d}
$$


where $I$ is the identity matrix, which the matrix $A$ is extremely close to. Reordering Equation 4.8 results in Equation 4.9, which can be iteratively solved to produce a divergence free vorticity field. The new particle strength vectors for iteration $n+1$ are equal to

$$
\boldsymbol{\alpha}_{p, n e w}^{n+1}=\boldsymbol{\alpha}_{p, \text { old }}+\boldsymbol{\alpha}_{p, \text { new }}^{n}-\sum_{q} \operatorname{vol}_{q} \boldsymbol{\alpha}_{q, \text { new }}^{n} \zeta_{\sigma}\left(\mathbf{x}_{p}-\mathbf{x}_{q}\right)
$$

where $v o l_{q}$ is the volume of particle $q$, calculated using Equation 4.1 .

The application of Equation 4.9 forced the discretized vortex particle vorticity field to be divergence free, as the theoretical vorticity field would be. A set number of iterations was selected, in this case $n_{i t e r}=15$. A convergence history is shown in Figure 4.4. The error is measured as the sum of the difference between the current iteration strength vector magnitude and the previous iteration value, $\sum\left|\alpha_{n e w}^{n+1}\right|-$ $\sum\left|\alpha_{\text {new }}^{n}\right|$. By the time the 15th iteration is reached, the difference between the current and past iteration becomes sufficiently small.

\subsection{Vortex Core Size Optimization}

The initial enstrophy condition described by Equation 4.4 was used to set the initial vortex core overlap value by ensuring that the initial kinetic energy decayed correctly. The vortex core overlap is a measure of how much the vortex cores of surrounding particles are overlapping each other, and is defined as $\sigma / h$, where $h$ is the typical distance between neighboring particles. A vortex core overlap value of greater than one implies that there is some overlap between vortex cores, which wouldn't 


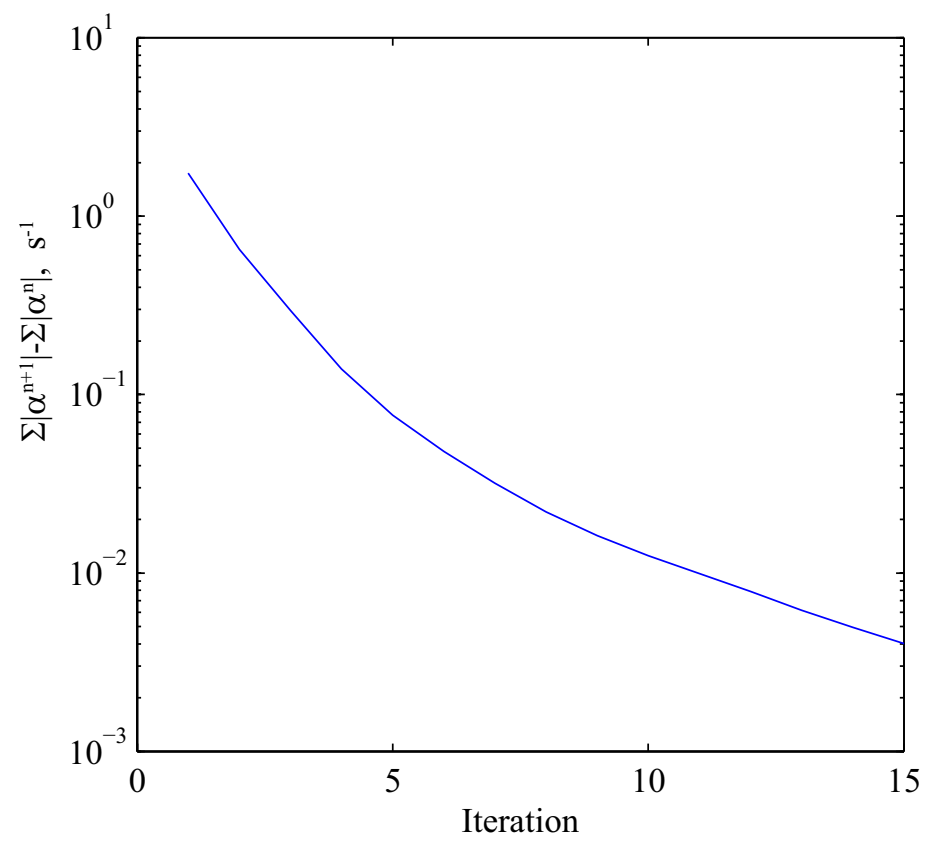

Figure 4.4: Convergence of particle strength vectors with Beale's Method iteration number.

exist if $\sigma / h \leq 1$. This core overlap is essential because the error in discretizing the vorticity field into particles has one term that is $\mathscr{O}\left(\sigma(h / \sigma)^{m}\right)$, where $m$ is related to the number of derivatives that can be taken of $\zeta_{\sigma}$, and is much greater than 1 for all practical regularizations. As such, for this error term to vanish to zero the value of $\sigma / h$ must be greater than one.

The core overlap was set to an overlap ratio of $\sigma / h=1.3$, which resulted in $\sigma=0.1$. Although it has been proven in convergence studies that $\sigma / h$ must be greater than one, there is no all encompassing answer for the exact value it should be. Winckelmans chose 1.3 so it is selected here to remain consistent with his work. From that initial estimate, the vorticity field was relaxed to ensure that the particle vorticity field represented the exact vorticity field as closely as possible. Next, two 
time steps were simulated, and the kinetic energy value was obtained after each step. The values were input into a second order forward difference to determine $\frac{d \tilde{E}(0)}{d t}$, which was then compared with the initial enstrophy condition. The finite difference can be written as

$$
\frac{d \tilde{E}(0)}{d t}=\frac{-\tilde{E}(2 \Delta t)+4 \tilde{E}(\Delta t)-3(\tilde{E}(0))}{2 \Delta t}
$$

The result of Equation 4.10 was compared with $-\nu \varepsilon(0)$, and if the two values weren't within a desired tolerance then $\sigma$ was adjusted slightly, and the steps were repeated. This provides a final, and strict condition for exactly setting the core radius.

\subsection{Third Order Low Storage Runge-Kutta Solver}

In addition to correctly matching all the initial conditions, to achieve a correct answer the scheme used for the time evolution of Equation 3.43 and Equation 3.44 was also matched. Winckelmans employed a unique third order Runge-Kutta solver first laid out by Williamson $\frac{41}{41}$ and investigated further by multiple authors. $\frac{42}{46}$ The scheme is unique for two reasons; it cancels the first order round-off error term normally present in Runge-Kutta schemes, and it requires storage equivalent to a typical first order Runge-Kutta solver.

\subsubsection{Round-off Error Removal}

The Williamson Runge-Kutta Scheme has first order round-off error removal. Butcher provides an excellent explanation of the round-off error removal technique $e^{47}$

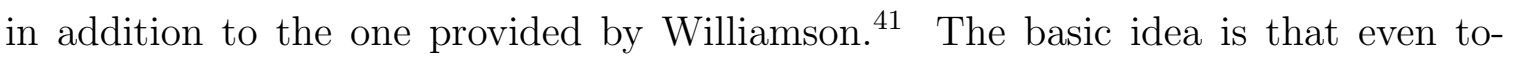


day's computers have finite precision, and therefore roundoff error is a certainty. The roundoff error for each step can be calculated as the difference between the current and previous function values and the intermediate function update term that updated them, that is

$$
e_{j}=\left(x_{j}-x_{j-1}\right)-b_{j} q_{j}
$$

where $e_{j}$ is the round-off error associated with stage $j$ of a single Runge-Kutta timestep solution, and $b_{j}$ is a factor that is dependent on which third order solver is selected. This roundoff error is not a constant value for a given substep, but rather must be recalculated based on the previous update of the function value.

Although Williamson provides a table with coefficient values to create many different Runge-Kutta solvers, the specific case used for this work was Case 7. For this case, the $b_{j}$ values of Equation 4.11 are $b_{1}=\frac{1}{3}, b_{2}=\frac{15}{16}$, and $b_{3}=\frac{8}{15}$. A fully expanded formulation of the Case 7 scheme by Williamson is shown in Equation 4.12 .

$$
\begin{gathered}
q_{1}=\tilde{h} f\left(x_{0}\right)-6 e_{3} \\
x_{1, \text { temp }}=x_{0}+\frac{1}{3} q_{1} \\
e_{1}=\frac{1}{3} q_{1}-\left(x_{1, \text { temp }}-x_{0}\right) \\
x_{1}=x_{1, \text { temp }} \\
q_{2}=\tilde{h} f\left(x_{1}\right)-\frac{10}{3} e_{1}-\frac{5}{9} q_{1} \\
x_{2, \text { temp }}=x_{1}+\frac{15}{16} q_{2}
\end{gathered}
$$




$$
\begin{gathered}
e_{2}=\frac{15}{16} q_{2}-\left(x_{2, \text { temp }}-x_{1}\right) \\
x_{2}=x_{2, \text { temp }} \\
q_{3}=\tilde{h} f\left(x_{2}\right)-\frac{15}{8} e_{2}-\frac{153}{128} q_{2} \\
x_{3, \text { temp }}=x_{2}+\frac{8}{15} q_{3} \\
e_{3}=\frac{8}{15} q_{3}-\left(x_{3, \text { temp }}-x_{2}\right) \\
x_{3}=x_{3, \text { temp }} .
\end{gathered}
$$

The $q_{j}$ terms are intermediate update values, while the $x_{j}$ terms are the updated function values. The $\tilde{h}$ term is the step size for the Runge-Kutta solver, with the tilde added to differentiate it from the $h$ associated with particle neighbor distances. Williamson presents the same solver, but in a more compact form by not displaying the temporary $x_{j}$ terms or the calculation of the $e_{j}$ terms. If additional clarity on the matter is sought, Butcher ${ }^{47}$ presents a comparison of a method with and without roundoff error for a simple first order Runge-Kutta solver.

The round-off error is completely negligible for moderate step sizes, but as the step size continues to be reduced, and the number of steps for the same solution is increased, the compounding of the round-off error can become quite significant. As an example, a third order Runge-Kutta solver was used to approximate $y=\sin (t)$ from 0 to 1 by solving the ODE $\frac{d y}{d t}=\sqrt{1-\sin (t)^{2}}$, first in single and then in double precision. The error between the exact solution and the approximate solution is measured at 1 for various step sizes. Figure 4.5 shows the resulting comparisons. 


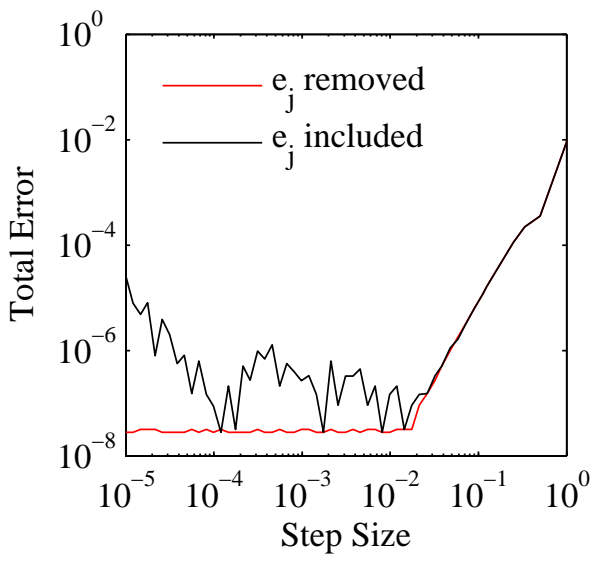

(a) Single Precision

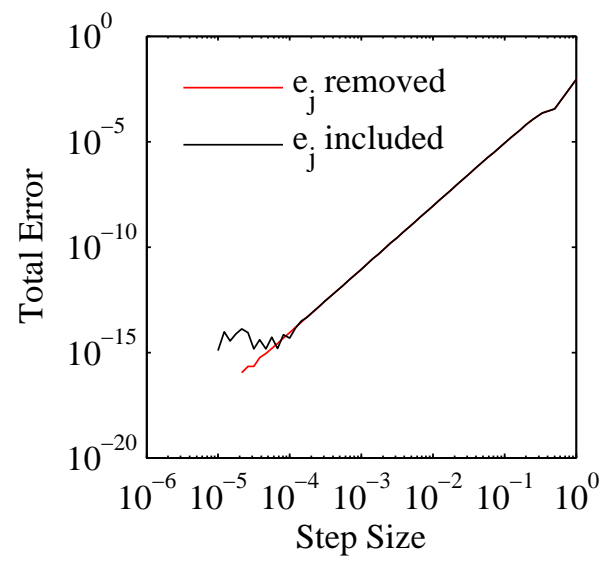

(b) Double Precision

Figure 4.5: Error associated with a third order Runge-Kutta approximation of the sine function, both with and without round-off error removal.

\subsubsection{Low Storage}

The low storage aspect of the Williamson Runge-Kutta solver was targeted specifically at $N$-body problems, where large body counts make Runge-Kutta schemes difficult from a storage sense, because a coefficient must be stored for every particle for each order in the solver in addition to the term the scheme is being applied to. For third order solvers there are a large number of possible coefficients that can be applied to the general Runge-Kutta scheme to achieve various desirable results, such as lowest round-off error or lowest truncation error. To obtain the low storage form of Case 7, the unique substep terms are no longer all stored throughout a step. Instead, a low storage form of Equation 4.12 can be seen in Equation 4.13 below.

$$
\begin{gathered}
q=\tilde{h} f\left(x_{0}\right)-6 e \\
x_{t e m p}=x_{0}+\frac{1}{3} q
\end{gathered}
$$




$$
\begin{gathered}
e=\frac{1}{3} q-\left(x_{t e m p}-x\right) \\
x=x_{\text {temp }} \\
q=\tilde{h} f(x)-\frac{10}{3} e-\frac{5}{9} q \\
x_{t e m p}=x+\frac{15}{16} q \\
e=\frac{15}{16} q-\left(x_{t e m p}-x\right) \\
x=x_{t e m p} \\
\tilde{h} f(x)-\frac{15}{8} e-\frac{153}{128} q \\
x_{t e m p}=x+\frac{8}{15} q \\
e=\frac{8}{15} q-\left(x_{t e m p}-x\right) \\
x=x_{t e m p} .
\end{gathered}
$$

The term low storage refers to the fact that as each consecutive $q$ and $x$ term is calculated, it can simply be stored over its predecessor, as is shown by the equation. This is a large reduction from a traditional third order Runge-Kutta solver, which would require storage of coefficients $k_{1}, k_{2}$, and $k_{3}$, as well as the function being updated, $x$, even without any roundoff error removal.

\subsection{Initial Particle Parameters}

Running the vortex ring simulation at $n_{c}=4$ results in $r_{l}=0.03889$ for a vortex ring radius of $R=1$. Additionally, this means that a total of $N_{s}=81$ particles are 
used to discretize a disk of the vortex ring, with a total of $N_{\varphi}=80$ disks composing the vortex ring model, for a total of $N=6,480$ particles. These values for particle count correspond to a core radius value of $\sigma=0.100$. The simulation was run using double precision for all variables.

Initial values for several of the diagnostics can be examined for the particle positions at time $t=0$. Obviously all of the validation values are slightly different from Table 4.1: Comparison of current vortex particle implementation with Winckelmans initial conditions $\stackrel{3}{[}$

\begin{tabular}{|c|c|c|}
\hline & Winckelmans & Verification \\
\hline$n_{c}$ & 4 & 4 \\
\hline$N$ & 6,480 & 6,480 \\
\hline$I(0)$ & 3.213919 & 3.1326 \\
\hline$\tilde{E}(0)$ & 1.047552 & 1.012823 \\
\hline$\tilde{E}_{f}(0)$ & 1.047634 & 1.012922 \\
\hline$\tilde{\varepsilon}(0)$ & 61.34640 & $\mathrm{~N} / \mathrm{A}$ \\
\hline$\tilde{\varepsilon}_{f}(0)$ & 62.38420 & 63.38210 \\
\hline$d \tilde{E} / d t(0)$ & -0.1276 & -0.1276 \\
\hline
\end{tabular}

the values calculated by Winckelmans. The values are consistently slightly different, which leads to the assumption that there is some slight difference between the two models. Additionally, no value is displayed for $\tilde{\varepsilon}(0)$ because there appeared to be an error in the equation provided to calculate it. Because all of the values in Table 4.1 are for $t=0$ the difference between the currently implemented vortex method and the work of Winckelmans must come during the discretization and strength assignment portion of the simulation, rather than during any time stepping, although additional differences could have occurred in the time evolution calculations. 
There are several places that these differences may have arisen before time evolution was started. First, Winckelmans indicates that $n_{\text {iter }}=5$ is typically enough to converge Beale's Method, but gives no indication of what value was used during his simulation. As all the values in Table 4.1 are dependent on particle strength vectors, all of the values are dependent on the iteration number selected. Additionally, it is unclear what programming language Winckelmans employed in his simulation, which means that subtle differences may exist in the definitions of trigonometric functions, values such as $\pi$, and single versus double precision calculations. Lastly, although Winckelmans indicates that the initial decay of kinetic energy should be used to adjust the vortex core radius value, he does not provide a radius value after the adjustment to compare to, so it is possible that a difference exists in the core radius values after adjustment.

\subsection{Time Evolution}

Once all the initialization of the simulation was done, the time evolution of the vortex ring was conducted using the third order Runge-Kutta scheme on Equation 3.43 and Equation 3.44 with the viscous diffusion term included. The diagnostics, which were computationally costly to calculate, were evaluated at set intervals of time steps to examine their time dependent variation. An important note is that the data from Winckelmans which is compared to in the following section is for $n_{c}=6$, which was the only data he presented. While this calculation was possible for Winckelmans 
due to use of supercomputers, the memory restrictions of the desktop made that impossible for the current vortex particle scheme. This is because Beale's Method requires the creation of an $N$ by $N$ matrix in order to relax the particle vorticity field to the true vorticity field, and with $n_{c}=6$ resulted in $N=19,773$, which was too large to preallocate a square matrix.

\subsubsection{Results}

There were a variety of results that were examined for the single vortex ring simulation. To check that the transpose scheme was implemented correctly, the total vorticity evolution over the length of the simulation was examined. As mentioned previously, the transpose formulation of stretching is the only one that conserves total vorticity, so if it is implemented correctly, no vorticity decay should occur. Figure 4.6 shows the evolution of both (a) total vorticity from Equation 3.45, and (b) angular impulse from Equation 3.49. Both diagnostics in Figure 4.6 are shown to be conserved, as theory indicates they should be.

In addition to examining the evolution of angular impulse and total vorticity, several other diagnostics were investigated, and compared to the results of Winckelmans. ${ }^{3}$ The data being compared to was digitized from the published paper, and then the data was smoothed using polynomial fits from MATLAB's polyfit to remove noise from digitization. For both the enstrophy and the kinetic energy there are two lines present in the original data, however in both cases the lines overlap because the particle vorticity field is still a good approximation of the true field, and hence 


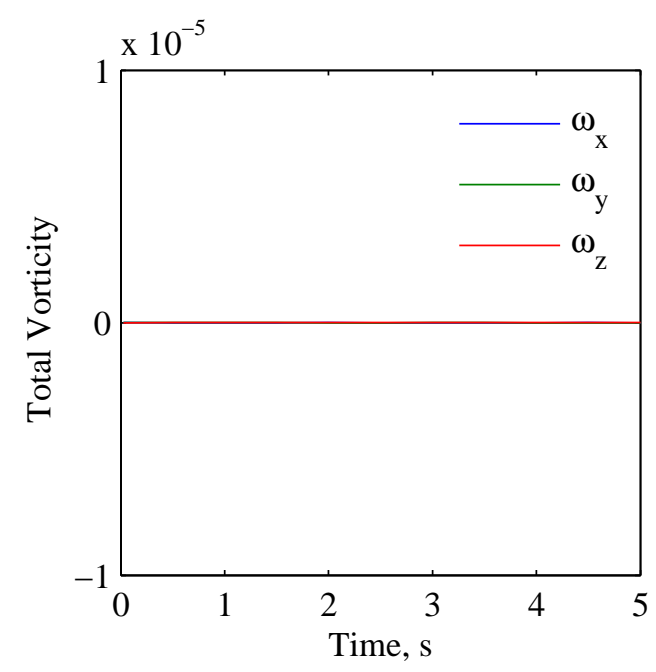

(a) Total Vorticity

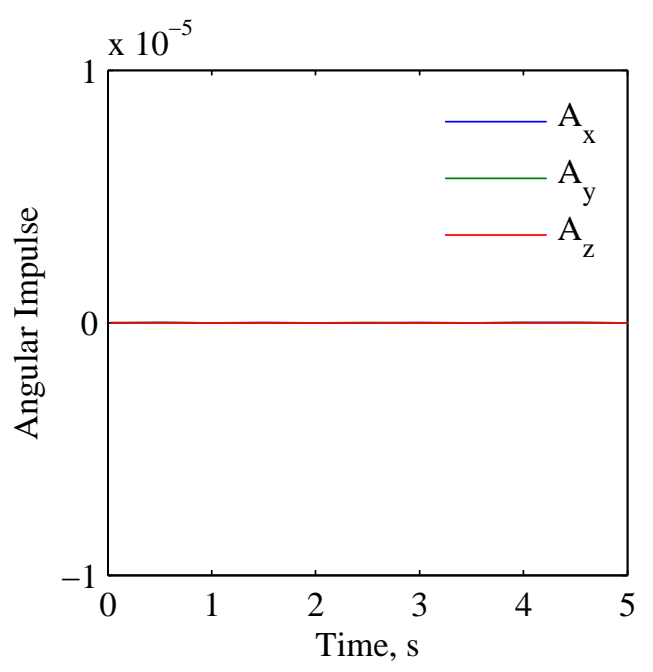

(b) Angular Impulse

Figure 4.6: Time dependent decay of (a) total vorticity, and (b) angular impulse using a particle discretization of a vortex ring.

$\tilde{E}(t)=\tilde{E}_{f}(t)$ and $\tilde{\varepsilon}(t)=\tilde{\varepsilon}_{f}(t)$. Because of this fact, only a single line is plotted to represent the Winckelmans data.

All of the following figures that are compared with specific plots from Winckelmans use the same axis scales as the figures that Winckelmans presented in his dissertation. After angular impulse and total vorticity the next diagnostic under consideration was the evolution of linear impulse from Equation 3.46. Theoretically linear impulse should be conserved throughout the simulation since it is a viscous unbounded flow. It is clear in Figure 4.7 that while the normalized linear impulse was conserved for approximately two seconds, after that it began to decay. Clearly the agreement between the evolution from Winckelmans, and that of the currently implemented vortex particle scheme is quite good.

The normalized enstrophy evolution from Equation 3.53 can be seen in Fig- 


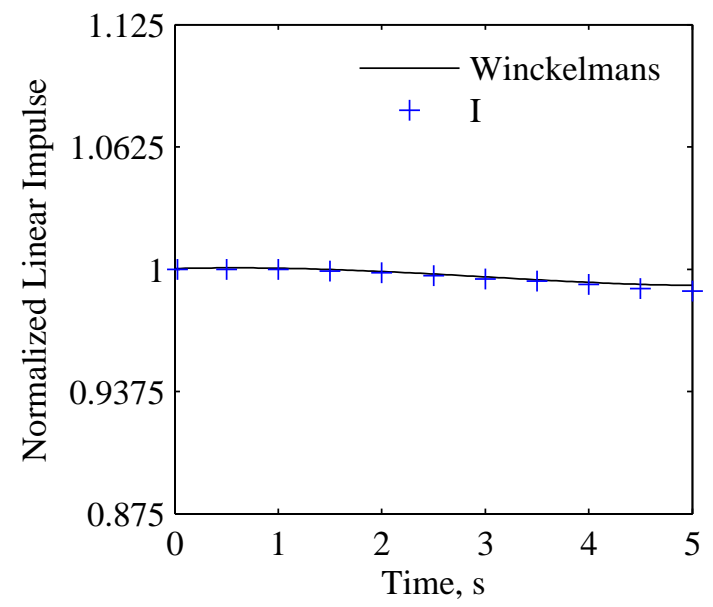

Figure 4.7: Time dependent linear impulse decay using a vortex particle discretization of a vortex ring.

ure 4.8. Again, the agreement between the evolution data from Winckelmans, and that of the currently implemented vortex particle scheme is excellent. No line is plotted for $\tilde{\varepsilon}(t)$, due to the same reason that no value appeared for its initial condition. There appears to be an error in the equation provided to solve, resulting in incorrect values during calculation.

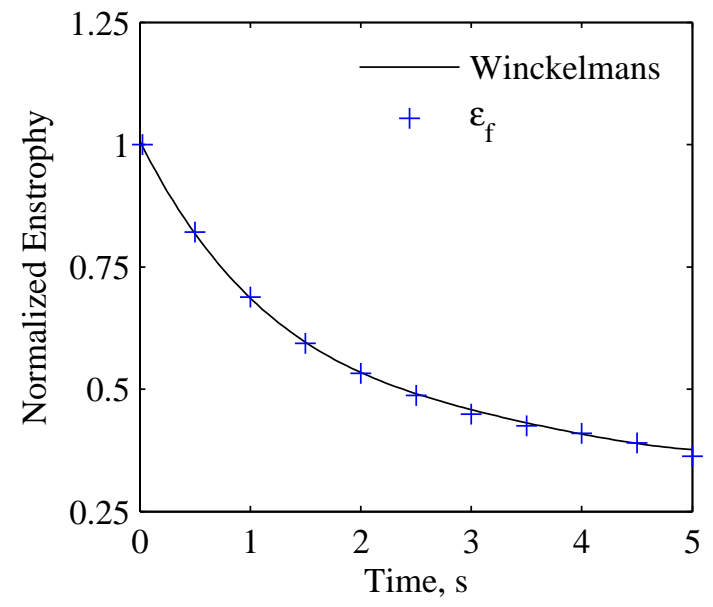

Figure 4.8: Time dependent enstrophy decay using a vortex particle discretization of a vortex ring.

Lastly, the evolution of the normalized kinetic energy from Equation 3.51 and 
Equation 3.52 were examined in Figure 4.9. The agreement between previous data and that calculated from the implemented vortex particle scheme is again very good. The high amount of agreement for each of these normalized terms throughout their evolution is a key indication that the vortex influencing equations for velocity and stretching, as well as the time evolution scheme employed, are correctly implemented.

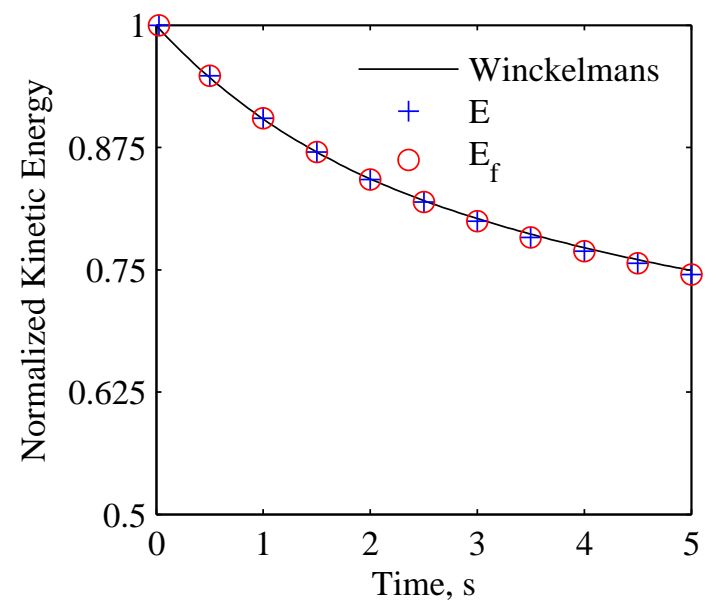

Figure 4.9: Time dependent kinetic energy decay using a vortex particle discretization of a vortex ring.

One last figure that can be examined is the vorticity contour plot in Figure 4.10 . This figure shows the breakdown of the vorticity contours at $t=5$ for a single disk of the vortex ring, relative to the initially circular contours at time $t=0$. Clearly the clean circular vortex structure from the initial particles has greatly decayed, although the general structure can still be seen in the final contours as well. Figure 4.10 matches well with the equivalent contour plot of Winckelmans for the single vortex ring simulation. 


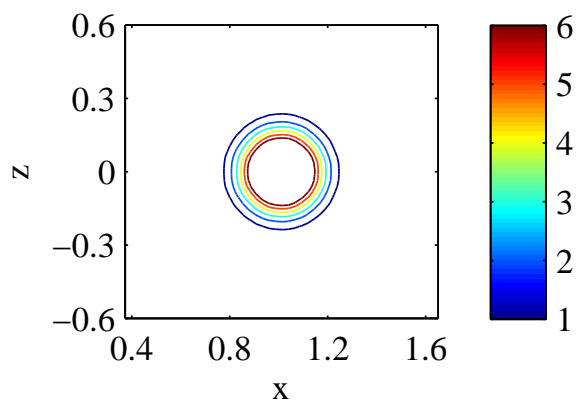

(a) $\mathrm{t}=0$

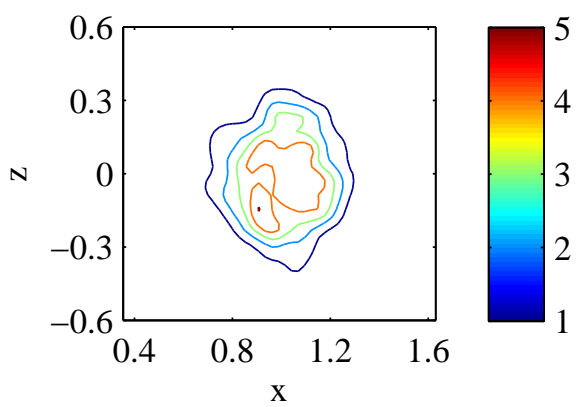

(b) $\mathrm{t}=5$

Figure 4.10: Contours of vorticity for both (a) the initial particle discretization of the ring and (b) the final solution at $t=5$ seconds. 


\section{Chapter 5}

\section{Analytical Actuator Disk Theory}

For the propeller-airfame interaction method to be viable, it was necessary to demonstrate that some formulation existed that would allow vortex particles to model the same effects captured in analytical actuator disk theory. The main source for analytical theory was work by Conway 15,480 To gain understanding of actuator disk theory the lightly loaded actuator disk model was reconstructed, and the heavily loaded model was reexamined as well. The heavily loaded theory proved to be a much better benchmark for creating a vortex particle actuator disk, as it was capable of modeling streamtube contraction, which is an inherent capability of the particle scheme. Once both flowfield theories were understood, the details of the total pressure jump associated with the disk were investigated, as the capture of these effects would be just as critical as the correct modeling of dynamic pressure due to velocity in matching real flow characteristics. 


\subsection{Lightly Loaded Actuator Disk}

The lightly loaded, linearized actuator disk theory is the most basic of those presented by Conway. The method uses the superposition of two separate vortex systems to capture all the effects present in actuator disk theory. The goals that motivated the development of the lightly loaded, linearized actuator disk theory are in line with those of this research. Namely, that existing methods at the time required detailed panel models of the propeller. This requirement makes preliminary design problems more difficult to analyze, because a detailed final selection on the propeller design would be required just to begin aerodynamic analysis.

The work of Conway is heavily based on that of Hough and Ordway, who initially

proposed relationships for the velocity field induced by an actuator disk. ${ }^{[1]}$ As Conway states, though it was possible to calculate the velocity field using their method, the integral formulations were too complicated to solve with anything but numerical integration. Conway instead sought to reformulate velocity field equations in such a manner that the integration could be carried out analytically. Through Conway's new method it is possible to achieve similar equations to those of Hough and Ordway, or else an entirely new formulation that provides analytical solutions, depending on the order of integration. 


\subsubsection{Theory}

The most fundamental building block of the Conway method, and the Hough and Ordway method, is the vortex ring, and the influence it exerts. The streamfunction and velocity field of a vortex ring can be expressed in terms of elliptic integrals as

$$
\begin{gathered}
\psi(r, z)=\frac{\Gamma(a r)^{1 / 2}}{2 \pi} Q_{1 / 2}\left(\omega_{1}\right), \\
V_{r}(r, z)=\frac{\Gamma\left(z-z^{\prime}\right) k_{1}\left[\left(2-k_{1}^{2}\right) E\left(k_{1}\right)-2\left(1-k_{1}^{2}\right) K\left(k_{1}\right)\right]}{8 \pi\left(1-k_{1}^{2}\right)\left(a r^{3}\right)^{1 / 2}} \\
V_{z}(r, z)=\frac{\Gamma k_{1}\left[k_{1}^{2}\left(a^{2}-r^{2}-z^{2}\right) E\left(k_{1}\right)+4 r a\left(1-k_{1}^{2}\right) K\left(k_{1}\right)\right]}{16 \pi\left(1-k_{1}^{2}\right)(r a)^{3 / 2}}
\end{gathered}
$$

where

$$
\begin{aligned}
k_{1} & \equiv\left(\frac{4 a r}{(a+r)^{2}+\left(z-z^{\prime}\right)^{2}}\right)^{1 / 2}, \\
\omega_{1} & \equiv 1+\frac{(a-r)^{2}+\left(z-z^{\prime}\right)^{2}}{2 a r},
\end{aligned}
$$

and $Q(x)$ is a Legendre function of the second kind, $E(k)$ is a complete elliptic integral of the second kind, $K(k)$ is a complete elliptic integral of the first kind, $\Gamma(\omega)$ is the Gamma function, $z^{\prime}$ is the $z$ location of the vortex ring, and $a$ is the radius of the ring. These equations represent an alternative to the singularity that traditionally appears when considering flow induced by a vortex ring as the ring itself is approached.

Much as vortex particle theory relies on the Biot-Savart law to describe the induced velocity field, actuator disk theory uses Biot-Savart to find instantaneous velocities in the field. In this case though, the velocity information is time averaged, and based on the time averaged vorticity distribution, which is constructed of two vortex systems, 
the vortex ring system and the vortex blade system. The term linearized in lightly loaded, linearized actuator disk theory refers to the fact that in the linear limit, the two vortex systems are completely independent, and therefore their influences can be solved separately and then summed.

\section{Vortex Ring System}

The first, and arguably most important vortex system, is the vortex ring system. This system is responsible for modeling the contraction and acceleration of the flow induced by an actuator disk. This means that the axial and radial velocity influences from the actuator disk are accounted for by the vortex ring system. For a uniform axial perturbation velocity the vortex ring system is composed of a vortex tube, which can be thought of as an axial distribution of vortex rings, with a strength distribution of $\gamma(z)$. For an arbitrary axial perturbation velocity profile, the system is generalized to a series of concentric tubes, modeled by concentric axial distributions of vortex rings, where now the strength only varies radially, and is defined by $\gamma(r)$. In either case, the vortex ring system conserves vorticity.

If the propeller being modeled is contra-rotating, or if swirl effects are not of interest, then the vortex ring system is the only system necessary to obtain the resultant field information. The strength of the vortex ring system is defined by $\gamma(z)$, which is the vortex density as a function of axial location. Applying this vortex ring strength to Equations 5.1 through 5.3 and integrating over the entire vortex ring distribution provides equations for the influence from the system. Applying the 
assumption that there is no slipstream contraction, and noting that Conway states $\gamma(z)$ is constant and equal to $U_{d}$, the downstream axial perturbation velocity, it is possible to analytically integrate with respect to $z^{\prime}$ first, and then $s$ gives solutions for the flowfield from a vortex ring system. The velocity influences are

$$
\begin{gathered}
V_{r}(r, z)=\frac{-U_{d}}{2 \pi}\left(\frac{R_{a}}{r}\right)^{1 / 2} Q_{1 / 2}(\omega), \\
V_{z}(r, z)=\frac{U_{d}}{4}\left[\frac{z}{\pi\left(R_{a} r\right)^{1 / 2}} Q_{-1 / 2}(\omega)+\Lambda_{0}(\beta, k)+2\right] \quad\left(r<R_{a}\right),
\end{gathered}
$$

and

$$
V_{z}(r, z)=\frac{U_{d}}{4}\left[\frac{z}{\pi\left(R_{a} r\right)^{1 / 2}} Q_{-1 / 2}(\omega)-\Lambda_{0}(\beta, k)\right] \quad\left(r>R_{a}\right),
$$

where $\Lambda(\beta, k)$ is the Hueman's Lambda function, $\beta$ is defined as

$$
\beta=\sin ^{-} 1\left[\frac{z}{\left(z^{2}+\left(R_{a}-r\right)^{2}\right)^{1 / 2}}\right],
$$

and $k$ and $\omega$ are

$$
\begin{gathered}
k \equiv\left(\frac{4 R_{a} r}{\left(R_{a}+r\right)^{2}+z^{2}}\right)^{1 / 2}, \\
\omega \equiv \frac{z^{2}+r^{2}+R_{a}^{2}}{2 r R_{a}}
\end{gathered}
$$

Conway states that although his method for deriving these equations was different from Hough and Ordway, the resulting equations for field velocity influence are the same. Conway also provides equations for the vector and scalar potential, which aren’t provided by Hough and Ordway $\underline{48}$ 


\section{Vortex Blade System}

Entirely independent of the vortex ring system is the vortex blade system. The complete system is composed of three related subsystems. One of the subsystems is a constant strength hub vortex along the axis of symmetry of the streamtube, from the center of the disk extending to downstream infinity. Additionally, there is a distribution of vorticity on the slipstream boundary equal in strength and opposite in direction to the hub vortex. Finally, there is a distribution of vorticity in the radial direction on the actuator disk. When these three subsystems are combined they create a vorticity conserving system. This system is devoted entirely to capturing the effects of swirl. The in-disk distribution represents the lifting lines of the propellers, while the axial distributions describe the root and tip vortices shed from each of those lifting lines.

The velocity field described by the vortex blade system is much more simple than the vortex ring distribution because it only describes swirl velocity. The equation for swirl velocity can be written as

$$
V_{\phi}(r, z)=\frac{\Gamma_{h u b}}{2 \pi r} \quad(r>R(z) \text { or } z<0)
$$

and

$$
V_{\phi}(r, z)=0 \quad(r<R(z) \text { and } z>0)
$$




\subsubsection{Actuator Disk with Elliptic Loading}

Much as the elliptic loading case is the simplest case for finite wing theory, the most basic example of Conway's lightly loaded, linearized actuator disk theory is the

elliptically loaded case. In this case the velocity field can be described without the use of any of the elliptic integral terms that appear in Equations 5.1 through 5.4 .

The velocity functions representing the flowfield are

$$
\begin{gathered}
V_{r}(r, z)=\frac{V_{z 0}|z|}{2 r}\left(\frac{1}{\tilde{\alpha}}-1\right)- \\
\frac{V_{z 0} r}{2 R_{a}} \sin ^{-1}\left[\frac{2 R_{a}}{\left(z^{2}+\left(R_{a}+r\right)^{2}\right)^{1 / 2}+\left(z^{2}+\left(R_{a}-r\right)^{2}\right)^{1 / 2}}\right], \\
V_{z}(r, z)=2 V_{z}(r, 0)+ \\
V_{z 0}\left(-\tilde{\alpha}+\frac{z}{R_{a}} \sin ^{-1}\left[\frac{2 R_{a}}{\left(z^{2}+\left(R_{a}+r\right)^{2}\right)^{1 / 2}+\left(z^{2}+\left(R_{a}-r\right)^{2}\right)^{1 / 2}}\right]\right) \quad(z \geq 0),
\end{gathered}
$$

and

$$
V_{z}(r, z)=V_{z 0}\left(\tilde{\alpha}+\frac{z}{R_{a}} \sin ^{-1}\left[\frac{2 R_{a}}{\left(z^{2}+\left(R_{a}+r\right)^{2}\right)^{1 / 2}+\left(z^{2}+\left(R_{a}-r\right)^{2}\right)^{1 / 2}}\right]\right) \quad(z<0)
$$

where $\tilde{\alpha}$ is a dimensionless parameter defined as

$$
\alpha=\left(\frac{\left(\left(R_{a}^{2}-r^{2}-z^{2}\right)^{2}+4 R_{a}^{2} z^{2}\right)^{1 / 2}+R_{a}^{2}-r^{2}-z^{2}}{2 R_{a}^{2}}\right)^{1 / 2}
$$

Equations 5.14 through 5.16 can be used to plot the velocity at various points in the flowfield. Figure 5.1 shows the radial velocity variation with radial location for several different axial survey locations. It shows that the highest amount of radial 


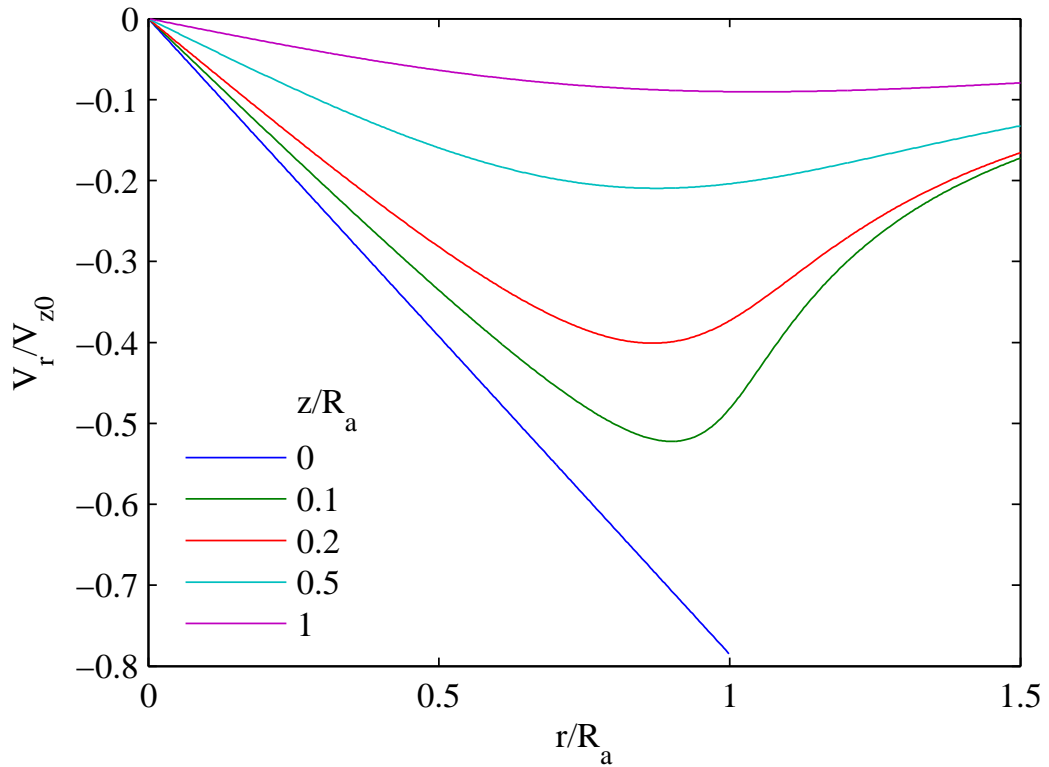

Figure 5.1: Radial variation of radial velocity at several axial locations (symmetric about $\mathrm{z}=0$ ).

velocity, which equates to streamtube contraction, occurs at the disk location, and as you travel farther downstream the radial velocity is reduced. This inherently makes sense because the streamtube reaches a final contracted radius at some downstream location. Also of note, the peak radial velocity always occurs at the slipstream boundary, while outside of the slipstream there is minimal radial velocity influence. The radial velocities are axially symmetrix about the disk, so Figure 5.1 only shows axial locations downstream.

Figure 5.2 shows the axial velocity variation with radial location at several different axial locations. In line with general actuator disk theory, it is apparent that at the disk, where $z=0$, the axial velocity is about half of what it will be downstream. Additionally, it is clear that slipstream contraction isn't explicitly modeled, because although the velocity influence outside the slipstream is reduced to nearly zero at 


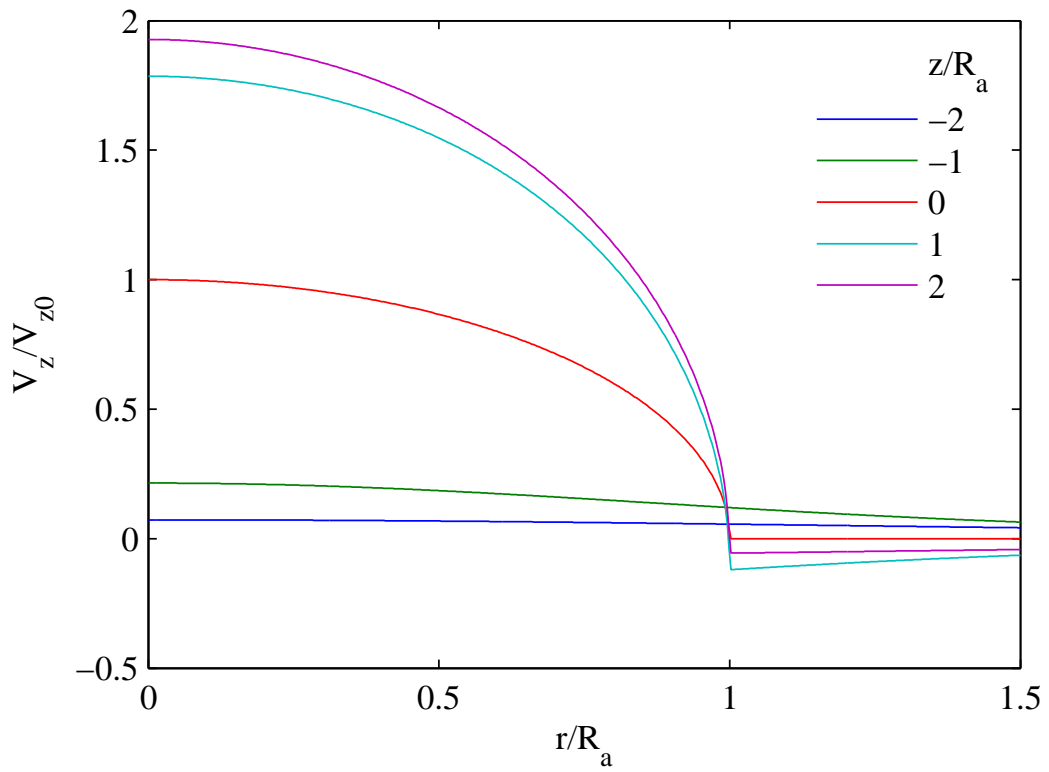

Figure 5.2: Radial variation of axial velocity at several axial locations.

each axial location, the slipstream boundary is still equal to the propeller radius, even several radii downstream of the disk. The elliptic velocity distribution is also clearly noticeable as the flow develops downstream.

\subsubsection{Actuator Disk with Parabolic Loading}

The parabolic loading case is slightly more complex than the elliptic case, but can still be expressed in terms of elliptic integrals, for which functions exist in MATLAB.

The velocity functions representing the flowfield are

$$
\begin{gathered}
V_{r}(r, z)=-\frac{V_{z 0}}{\pi}\left(\frac{r}{R_{a}}\right)^{1 / 2}\left[\frac{4 r}{R_{a}}-\frac{8\left(2-k^{2}\right)}{3 k^{2}}\right] \frac{E(k)}{k} \\
-\frac{V_{z 0}}{\pi}\left(\frac{r}{R_{a}}\right)\left[\frac{\left(4-k^{2}\right)\left(4-3 k^{2}\right)}{3 k^{4}}-\frac{r^{2}}{R_{a}^{2}}\right] k K(k) \\
-\frac{V_{z 0} r}{R_{a}^{2}}\left(z \Lambda_{0}(\beta, k)-2|z|\right) \quad\left(r<R_{a}\right),
\end{gathered}
$$




$$
\begin{gathered}
V_{r}(r, z)=-\frac{V_{z 0}}{\pi}\left(\frac{r}{R_{a}}\right)^{1 / 2}\left[\frac{4 r}{R_{a}}-\frac{8\left(2-k^{2}\right)}{3 k^{2}}\right] \frac{E(k)}{k} \\
-\frac{V_{z 0}}{\pi}\left(\frac{r}{R_{a}}\right)\left[\frac{\left(4-k^{2}\right)\left(4-3 k^{2}\right)}{3 k^{4}}-\frac{r^{2}}{R_{a}^{2}}\right] k K(k) \\
+\frac{V_{z 0} r}{R_{a}^{2}} z \Lambda_{0}(\beta, k)\left(r>R_{a}\right), \\
V_{z}(r, z)=\frac{V_{z 0}}{R_{a}^{2}}\left(R_{a}^{2}-r^{2}-2 z|z|\right)+\frac{6 V_{z 0} z}{\pi}\left(\frac{r}{R_{a}^{3}}\right)^{1 / 2} \frac{E(k)}{k} \\
+V_{z 0}\left[-\frac{z k\left(4 r^{2}\right) K(k)}{2 \pi\left(r R_{a}^{5}\right)^{1 / 2}}+\left(\frac{\left|R_{a}^{2}-r^{2}\right|+2 z^{2}}{2 R_{a}^{2}}\right) \Lambda_{0}(\beta, k)\right]\left(r<R_{a}\right),
\end{gathered}
$$

and

$$
\begin{gathered}
V_{z}(r, z)=\frac{6 V_{z 0} z}{\pi}\left(\frac{r}{R_{a}^{3}}\right)^{1 / 2} \frac{E(k)}{k} \\
+V_{z 0}\left[-\frac{z k\left(4 r^{2}\right) K(k)}{2 \pi\left(r R_{a}^{5}\right)^{1 / 2}}-\left(\frac{\left|R_{a}^{2}-r^{2}\right|-2 z^{2}}{2 R_{a}^{2}}\right) \Lambda_{0}(\beta, k)\right] \quad\left(r>R_{a}\right) .
\end{gathered}
$$

The radial velocity variation from Equation 5.18 and Equation 5.19 depends on whether the survey point is inside or outside the slipstream. The results are shown in Figure 5.3 for several axial locations along the streamtube. The results are relatively similar to those of the elliptic loading in Figure 5.1, although the magnitudes of radial velocity influence are smaller, particularly nearest to the disk. Additionally, there is no longer a linear change in radial velocity at the disk as the radial location is traversed from the hub to the tip.

Likewise, the axial velocity variation from Equation 5.20 and Equation 5.21 is plotted in Figure 5.4. Again the results are similar to those of the elliptic loading case. The distribution is clearly parabolic, rather than elliptic as in Figure 5.2, but the overall trends remain. Again no streamtube contraction is captured, shown by 


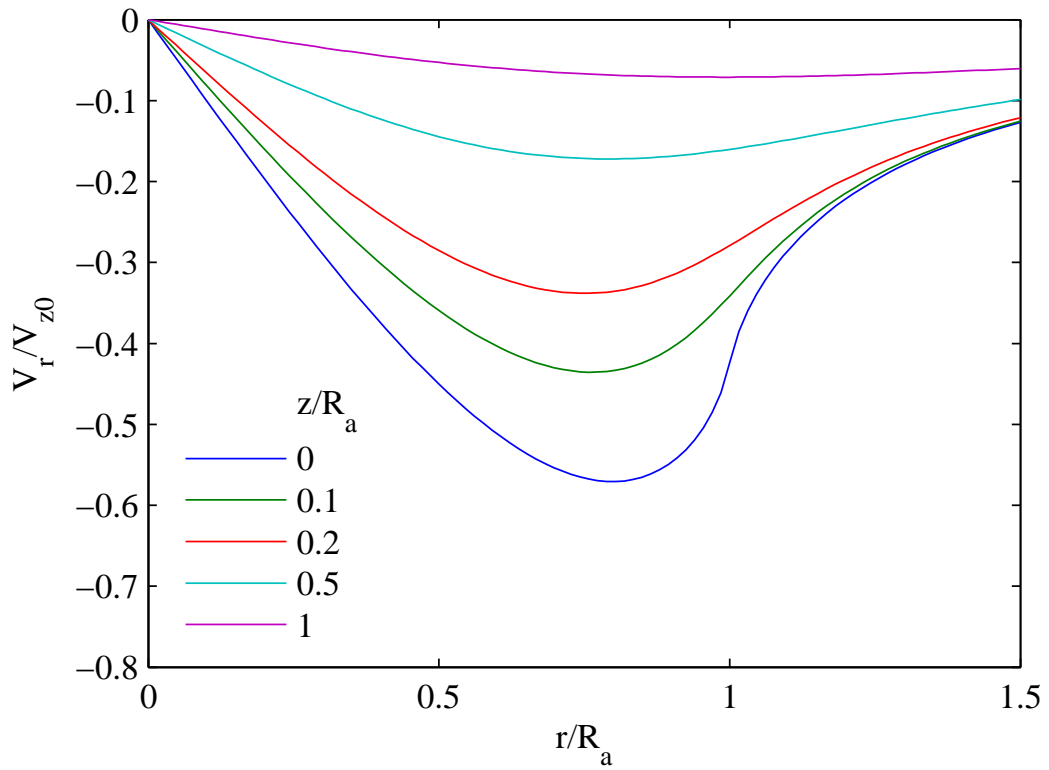

Figure 5.3: Radial variation of radial velocity at several axial locations (symmetric about $\mathrm{z}=0$ ).

the axial velocity influence returning to zero at $r / R_{a}=1$, even at the downstream axial survey locations. In the case of the parabolic loading, the velocity magnitude downstream doesn't increase as much as the elliptic loading relative to the velocity at the disk. Additionally, the velocity upstream of the disk is slightly larger than in the elliptic case. This demonstrates a slower transition both from the freestream condition to the conditions at the disk, and from the conditions at the disk to the conditions at an infinite distance downstream.

\subsection{Heavily Loaded Actuator Disk}

The particle scheme inherently accounts for streamtube contraction through time evolution, where calculations for future particle positions take into account the new positions of the current time step. This fact makes lightly loaded actuator disk theory 


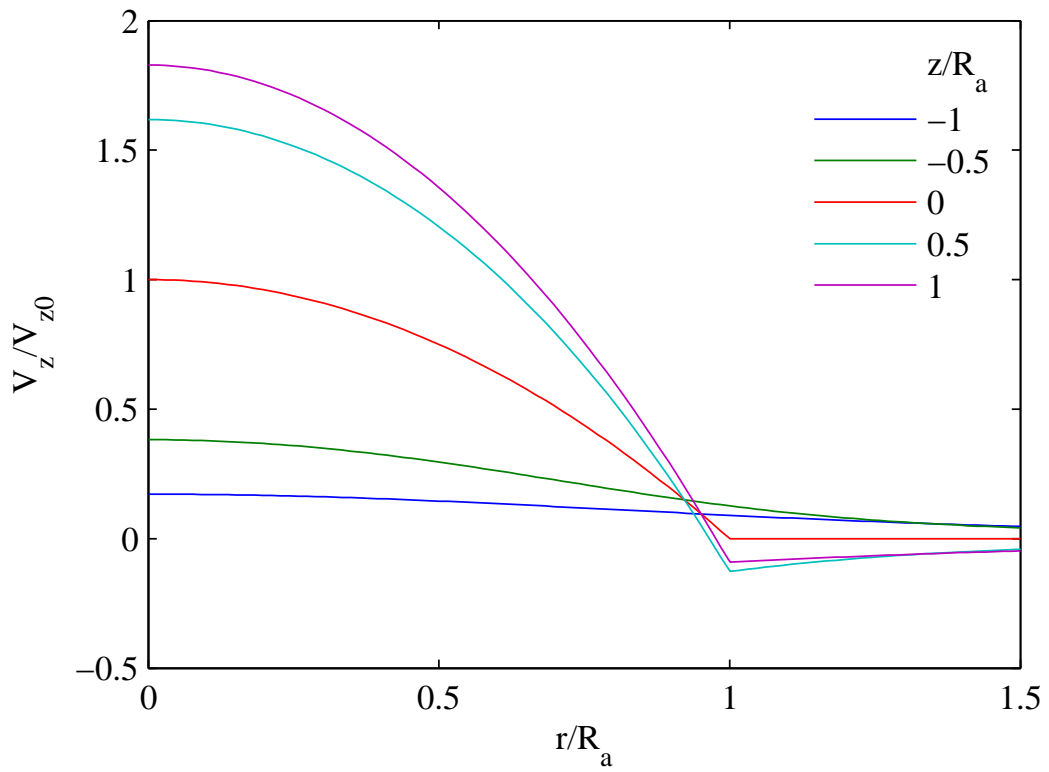

Figure 5.4: Radial variation of axial velocity at several axial locations.

less appropriate to compare the particle method with, and so the more complex heavily loaded actuator disk theory was also investigated $\underline{50}$

\subsubsection{Theory}

The heavily loaded actuator disk method is no longer completely analytical, but it is capable of taking slipstream contraction fully into account. The relationships are still functions of elliptic integrals, but in this case the elliptic integrals are inside a one-dimensional integral, rather than in an explicit formulation. Additionally, in heavily loaded theory the required numerical integration makes unbounded radial gradients infeasible in the load distribution. This means that the elliptic loading is no longer possible to analyze, as it has an infinite load gradient at the tip.

Again the heavily loaded theory is based on a system of vortex rings, just as in 
the lightly loaded case. For the heavily loaded case however, the simplification can no longer be made that the streamtube radius is constant at all axial locations. Instead, the term $R(z)$ must remain in the integral equations, unlike in lightly loaded theory.

The first step in the method is to determine the vorticity in the slipstream, unless vorticity is a known input. The solution is quite complex, although it can be simplified for the case of a contra-rotating propeller.

When vorticity is known the streamfunction must be calculated to gain information about all the flowfield characteristics. The streamfunction is solved for iteratively, because the unknown streamtube radius function $R(z)$ is part of the streamfuncion equation, written as

$$
\Psi\left(R_{a}, 0\right)=\frac{U_{\infty} R^{2}(z)}{2}+\frac{a R(z)}{2} \int_{0}^{\infty} R^{2}\left(z^{\prime}\right) I_{(-1,2,1)}\left(R\left(z^{\prime}\right), R(z), z-z^{\prime}\right) d z^{\prime} .
$$

In Equation 5.22 the term $a$ refers to the known change in enthalpy in the streamfunction. As a simple example of a known change in enthalpy, the change is defined as

$$
\frac{d h}{d \Psi}=-a,
$$

where a non-dimensional term involving $a$ can be written as

$$
\hat{a}=\frac{a R_{a}^{2}}{U_{\infty}}
$$

Conway states that Equation 5.22 converges quickly for low values of $\hat{a}$, specifically, values below 5 , while values higher than that tend to require under-relaxation. When 
the vorticity distribution is known to be linear, the term $\hat{a}$ is related to the thrust coefficient of the actuator disk through the relationship

$$
C_{T}=\frac{\hat{a}}{2}\left(\frac{R_{d}}{R_{a}}\right)^{4}\left[1+\frac{\hat{a}}{3}\left(\frac{R_{d}}{R_{a}}\right)^{2}\right]
$$

Because $\hat{a}>5$ requires under-relaxation, solving for $C_{T}$ for the parabolic loading case using Equation 5.25 shows that the convergence of Equation 5.22 requires underrelaxation for thrust coefficients over 3.147.

Conway's heavily loaded actuator disk method was not implemented in MATLAB, unlike the lightly loaded theory. Conway provided the data that was used to generate several of the figures in his heavily loaded actuator theory research. $\underline{52}$

\subsubsection{Actuator Disk with Parabolic Loading}

As mentioned earlier, the infinite gradient in the elliptical loading distribution makes it an infeasible case for the heavily loaded method. As a consequence, only the parabolic loading case is examined here, comparable to Figures 5.3 and 5.4 , except now without the lightly loaded assumption in place. Figure 5.5 shows the heavily loaded radial perturbation velocity influence. In this case the velocity is normalized by freestream velocity, rather than by $V_{z 0}$ as was the case for the lightly loaded fig-

ures. Radial velocity influence is now examined both upstream and downstream of the actuator disk because influence is no longer axially symmetric around the disk. This asymmetry stems from the streamtube contraction, which is now present in the heavily loaded model. While surveys at comparable distances upstream and down- 


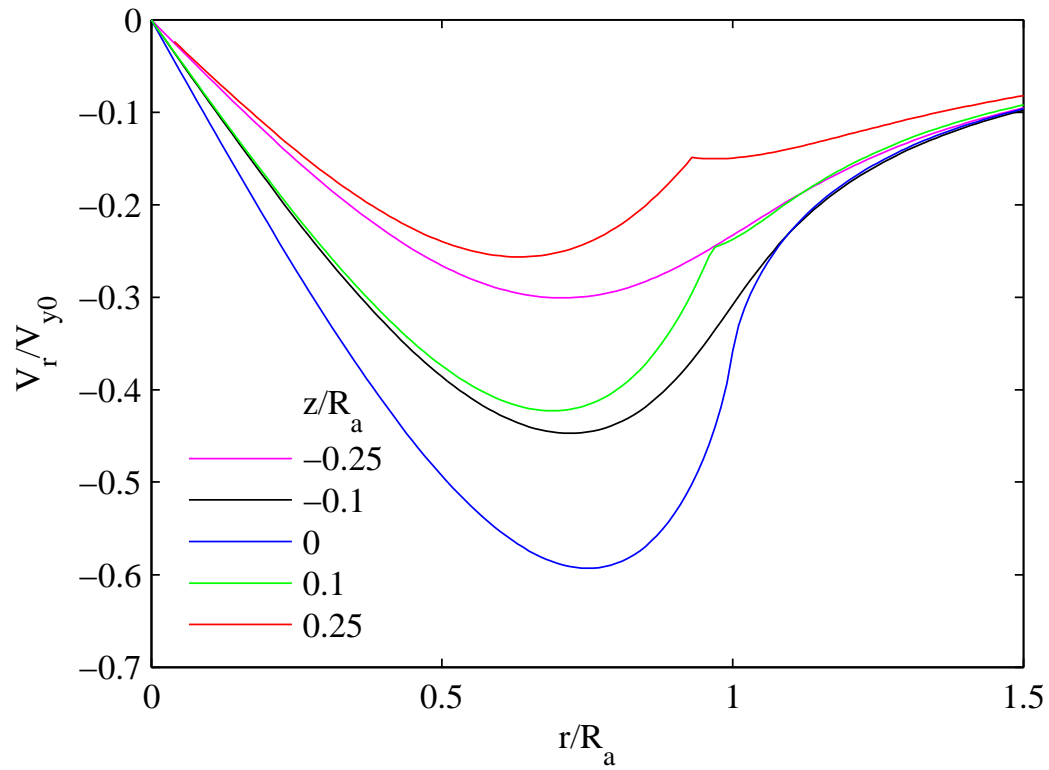

Figure 5.5: Radial variation of radial velocity at several axial locations.

stream yield similar magnitudes of velocity influence, there is a clear contraction effect present. The downstream survey locations have clear discontinuities at the slipstream boundaries. Similar to the lightly loaded case however, the largest magnitude of radial velocity influence occurs at the disk, with the influence reducing as distance increases either upstream or downstream. This is quite natural, as it demonstrates that at the farfield conditions the slipstream radius is converged, either at its freestream radius or its completely contracted radius.

Figure 5.6 shows the axial perturbation velocity influence for the heavily loaded theory. Again, the velocity influence is normalized by freestream velocity, which is why the velocity at $r=0$ and $z=0$ is no longer exactly one. The overall trends of Figure 5.5 are nearly identical to its lightly loaded counterpart, with one major exception occuring at the propeller tip. It is quite clear from the heavily loaded 


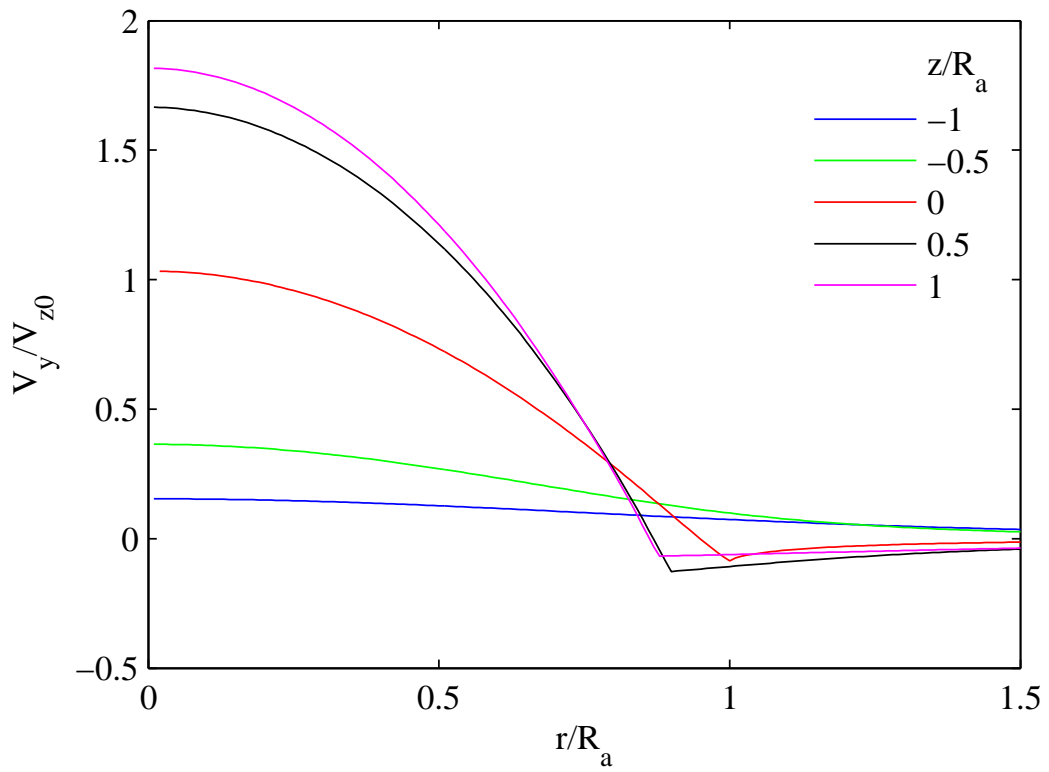

Figure 5.6: Radial variation of axial velocity at several axial locations.

figure that slipstream contraction is accounted for in this model. The discontinuity in velocity influence for $z=0$ occurs at $r / R_{a}=1$, indicating that the streamtube boundary occurs at $r=R_{a}$. Downstream however, we see the discontinuity appear at increasingly smaller values of $r / R_{a}$, indicating that the streamtube has contracted at $z=0.5$, and further at $z=1.0$. These contraction effects were not present in the lightly loaded theory, but are inherent in the vortex particle scheme.

\subsection{The Actuator Disk Total Pressure Jump}

Traditionally, pressure coefficients in a panel code are compared through change in dynamic pressure because the stagnation pressure is constant throughout the flowfield. The freestream pressure condition is written as

$$
p_{o, \infty}=p_{\infty}+q_{\infty}
$$


while the survey point condition would be

$$
p_{o}=p+q,
$$

where $p_{o, \infty}=p_{o}$ because the resulting pressure if the flow was adiabatically brought to rest anywhere in the field would be the same. The definition of the pressure coefficient in this case is

$$
C_{p}=\frac{p-p_{\infty}}{q_{\infty}}
$$

Solving Equation 5.26 for $p_{\infty}$ and Equation 5.27 for $p$, and plugging the resulting equations into Equation 5.28 yields

$$
C_{p}=\frac{p_{o}-q-\left(p_{o, \infty}-q_{\infty}\right)}{q_{\infty}} .
$$

Because, as was stated, the stagnation pressure is equal everywhere in the field, the stagnation terms cancel out resulting in

$$
C_{p}=\frac{q_{\infty}-q}{q_{\infty}},
$$

or, more simply, when the flow is incompressible so that $\rho$ is constant,

$$
C_{p}=1-\left(\frac{U}{U_{\infty}}\right)^{2} .
$$

Equation 5.31 is a frequently used relationship for pressure coefficient in many aerodynamic flow solutions, however it is no longer valid in the presence of an actuator disk, because $p_{o}$ is not constant.

With the addition of the actuator disk, the stagnation pressures are now different inside the streamtube downstream of the disk compared with everywhere else. This 
fact means that Equation 5.31 is no longer valid, and a more general relationship must be used if pressure coefficient information is desired. The actuator disk causes a discontinuity in the pressure field to occur, adding a $\Delta p$ at the disk location. This means that downstream of the actuator disk inside the streamtube the stagnation pressure is $\Delta p_{o}$ different from the stagnation pressure elsewhere in the field. Thus, the stagnation pressure terms in Equation 5.29 no longer cancel out.

In a extremely simple case a uniform pressure jump at all radial stations could be employed, but this scenario is not realistic. Rather, a pressure jump distribution as a function of radial location, $\Delta p_{o}(r)$, can be used, where the distribution can be changed to fit any real propeller configuration. This means the pressure coefficient equation is now

$$
C_{p}=\frac{p_{o}-q-\left(p_{o, \infty}-q_{\infty}\right)}{q_{\infty}}=1-\left(\frac{U}{U_{\infty}}\right)^{2}+\left(\frac{\Delta p_{o}}{q_{\infty}}\right),
$$

where the value of $\Delta p_{o}$ depends on the radial location if the survey point is inside the streamtube and behind the actuator disk, and is zero outside of the streamtube or upstream of the disk.

The change in stagnation pressure in the streamtube is related to the pressure jump that occurs at the disk. This is because the velocity change is continuous across the disk, while the pressure change is discontinuous. Thus, the velocity change in the infinitesimal limit across the disk is zero, while the pressure change is not, and the 
total pressure change from one side of the disk to the other is

$$
\Delta p_{o}=p_{o}^{+}-p_{o}^{-}=\left(p^{+}+q^{+}\right)-\left(p^{-}+q^{-}\right)=\left(p^{+}-p^{-}\right)=\Delta p .
$$

Therefore, knowledge of the pressure change at the disk can provide information about the total pressure change. Additionally, total pressure is constant along a streamline, so the total pressure change at any point in the field can be determined by tracing a streamline back to the disk and determining the total pressure change at the disk.

The $\Delta p$ term of the actuator disk is related to the loading and the thrust the disk produces. As Lotstedt ${ }^{7}$ states

$$
\Delta p=\frac{d T}{2 \pi r d r}
$$

Additionally, we know from actuator disk theory that

$$
d T=2 \pi \rho r\left(V_{z}(r, \infty)\left(U_{\infty}+V_{z}(r, \infty)\right)-\frac{V_{\phi}^{2}(r, \infty)}{2}\right) d r .
$$

Plugging Equation 5.35 into Equation 5.34 gives a relationship for $\Delta p$ in terms of the velocity profile at downstream infinity

$$
\Delta p=\rho\left[V_{z}(r, \infty)\left(U_{\infty}+V_{z}(r, \infty)\right)-\frac{V_{\phi}^{2}(r, \infty)}{2}\right] .
$$

Using the linearized actuator disk theory, where $V_{z}(r, \infty)=2 V_{z}(r, 0)$, results in

$$
\Delta p=\rho\left[2 V_{z}(r, 0)\left(U_{\infty}+V_{z}(r, 0)\right)-\frac{V_{\phi}^{2}(r, \infty)}{2}\right] .
$$

Finally, assuming a contra-rotating actuator disk with no swirl results in

$$
\Delta p=2 \rho\left[V_{z}(r, 0)\left(U_{\infty}+V_{z}(r, 0)\right)\right] .
$$


Thus, depending on the assumptions that are suitable to make, we have a relationship that describes the pressure jump at the disk. It is convenient to change these relationships to pressure coefficient jumps because it eliminates the requirement for a density being specified. The pressure coefficient jump is simply equal to the pressure jump divided by the freestream dynamic pressure,

$$
\Delta C_{p}=\frac{1}{\frac{1}{2} U_{\infty}^{2}}\left[V_{z}(r, \infty)\left(U_{\infty}+V_{z}(r, \infty)\right)-\frac{V_{\phi}^{2}(r, \infty)}{2}\right] .
$$

With the linearized actuator disk theory assumption we have

$$
\Delta C_{p}=\frac{1}{\frac{1}{2} U_{\infty}^{2}}\left[2 V_{z}(r, 0)\left(U_{\infty}+V_{z}(r, 0)\right)-\frac{V_{\phi}^{2}(r, \infty)}{2}\right] .
$$

The contra-rotating actuator disk assumption yields

$$
\Delta C_{p}=\frac{2}{\frac{1}{2} U_{\infty}^{2}}\left[V_{z}(r, 0)\left(U_{\infty}+V_{z}(r, 0)\right)\right] .
$$

The most critical location requiring knowledge of the actuator disk pressure jump is on the surface of the geometry, as this is where the most detailed experimental information was measured via surface pressure taps. The pressure jump here was easy to measure, with the first step being to use the specified velocity distribution at the disk to determine the total velocity along the surface of the nacelle. This velocity and the freestream velocity are the only terms required to find the pressure coefficient jump via Equation 5.41. This method is valid because the surface of the nacelle is a streamline passing through the disk root, and the pressure jump is constant along a streamline from the disk to downstream infinity. It becomes much more difficult to 
use this simple assumption when a wing is also involved, because the wing occupies an entire series of radial values inside the streamtube, rather than just one point at the root as the nacelle alone did.

\subsubsection{Effect of Efficiencies on Pressure Jump}

Unfortunately, the pressure coefficient jump described in Equation 5.41 is a function of the actuator disk efficiency, and in no way reflects the efficiencies of the real propeller being simulated. To achieve correct magnitudes, the inherent actuator disk efficiency must be removed, returning the efficiency to $100 \%$, and then the propeller efficiency must be applied instead.

The actuator disk efficiency can be calculated with relative accuracy as only a function of thrust coefficient. Von Mises states the relationship is

$$
\eta_{A D}=\frac{2}{1+\sqrt{1+C_{T}}}
$$

where $\eta_{A D}$ is the efficiency of the actuator disk. $\frac{53}{5}$ Thus, the $\Delta C_{p}$ term from Equation 5.40 is divided by $\eta_{A D}$ to return the solution to $100 \%$ efficiency.

Next, the propeller efficiency is calculated and applied. Propeller efficiency can be written as

$$
\eta_{P}=J \frac{C_{T}}{C_{p}},
$$

where $J$ is the advance ratio, and $C_{p}$ is the power coefficient. The $\Delta C_{p}$ that was previously divided by $\eta_{A D}$ is now multiplied by $\eta_{P}$ to achieve correct scaling for a real propeller case. 
As a simple validation of the method, the experimental velocity profile at $x=$ $525 \mathrm{~mm}$ was used with Equation 5.41, and then compared to the $\Delta C_{p}$ profile reported in AGARD from the experimental five hole probe sweep? $9[22] 23$ This allowed for the most direct possible assessment of Equation 5.40 because the variables entered in the equation were from the experimental data, and the data compared to was also from the experiment. Figure 5.7 shows the absolute distributions of $\Delta C_{p}$ from both experimental and calculated sources, as well as the error of the calculated method relative to experimental.

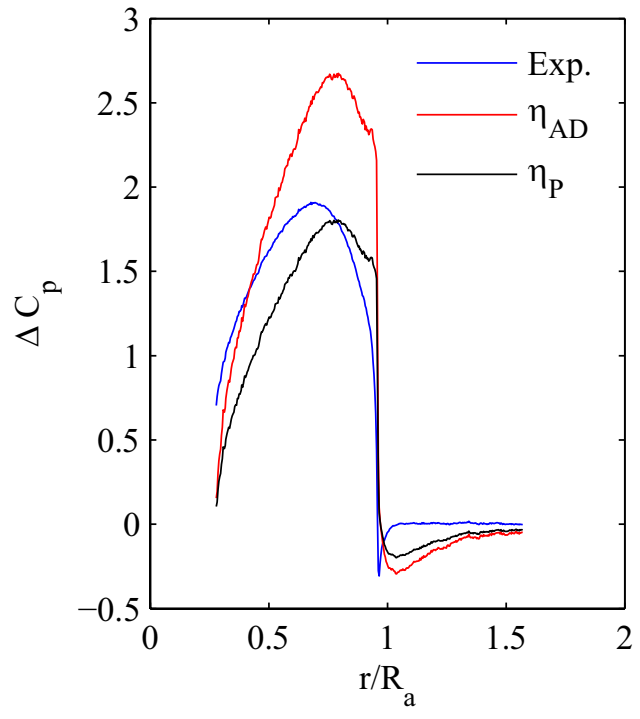

(a) $\Delta C_{p}$ Jumps

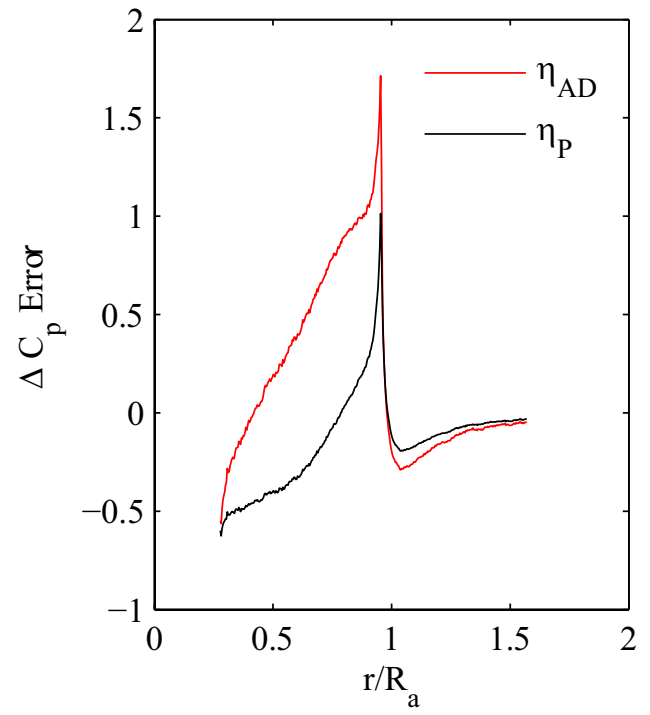

(b) $\Delta C_{p}$ Error

Figure 5.7: $\Delta C_{p}$ jump for the AGARD experiment (exp.) along with calculated $\Delta C_{p}$ at two different efficiency levels, actuator disk efficiency (AD) and propeller efficiency $(p)$, and error from experimental values.

The results from Figure 5.7 indicate a relatively good agreement between the experimental data and the calculated data when $\eta_{P}$ is applied, however the agreement is by no means exact. As such, for the AGARD validation case experimental $\Delta C_{p}$ 
values on the surface were used to ensure the most accurate comparison of the numerical scheme presented here. In preliminary design exercises however, the method described by Equation 5.40 should prove viable enough when experimental data is lacking, otherwise the path integral method presented in the next section can be used.

\subsubsection{Method of Implementation}

The calculation of pressure coefficient jump due to the actuator disk is more complex when the survey point is in the field rather than on the surface. Shapiro's formulation ${ }^{10}$ of Crocco's Theorem for an incompressible and inviscid fluid flow is reproduced from Equation 1.1,

$$
\mathbf{V} \times \boldsymbol{\omega}=\frac{1}{\rho} \nabla p_{o}
$$

where $p_{o}$ is the stagnation pressure. The cross product term in Equation 1.1 shows that the gradients of stagnation pressure are everywhere perpendicular to both the velocity and vorticity vectors, and therefore $p_{o}$ is constant along streamlines and

vortex lines $\frac{10}{10}$ A surface can be constructed which the velocity and vorticity vectors are always tangent to, and consequently is a surface of constant $p_{o}$. These surfaces are called Lamb surfaces, $\underset{54}{5}$ and they provide a method to determine the pressure jump anywhere in the field.

To understand the pressure jump at some point inside the streamtube, integration can be completed through all of the Lamb surfaces until the freestream stagnation condition is reached outside of the streamtube. This integration determines the 
change in stagnation pressure between the point of interest inside the streamtube and the freestream stagnation pressure where the integral is terminated. The integral is written as

$$
\Delta p_{o}=\int \nabla p_{o} \cdot d \mathbf{s},
$$

following the path described the the vector $\mathbf{s}$. Because the field being investigated is a scalar field, the integral is path independent, and thus any vector path s could be chosen. For simplicity, select $\mathbf{s}$ to always be parallel to $\nabla p_{o}$, and equivalently, always perpendicular to the Lamb surfaces. Thus

$$
d \mathbf{s}=\frac{\nabla p_{o}}{\left\|\nabla p_{o}\right\|} d s
$$

The integral in Equation 5.44 can then be rewritten as

$$
\Delta p_{o}=\int \frac{\nabla p_{o} \cdot \nabla p_{o}}{\left\|\nabla p_{o}\right\|} d s=\int \frac{\left\|\nabla p_{o}\right\|^{2}}{\left\|\nabla p_{o}\right\|} d s=\int\left\|\nabla p_{o}\right\| d s .
$$

To obtain a useful relationship from Equation 5.46, the pressure jump term needs to be related to vorticity, which is a value that can easily be found anywhere in the flow. Returning to Crocco's Theorem, the stagnation pressure gradient can be written as

$$
\nabla p_{o}=\rho(\mathbf{V} \times \boldsymbol{\omega}),
$$

and so the final form of the integral becomes

$$
\Delta p_{o}=\int \rho\|\mathbf{V} \times \boldsymbol{\omega}\| d s .
$$


As with the previously described on-surface pressure jump, the stagnation pressure coefficient jump is a more useful term, and requires dividing by the freestream dynamic pressure, resulting in

$$
\Delta C_{p o}=\frac{2}{U_{\infty}^{2}} \int\|\mathbf{V} \times \boldsymbol{\omega}\| d s
$$

The Lamb surfaces provide a great deal of generality to the method. The constant $\nabla p_{o}$ surfaces mean that the path of the integral can temporarily travel any distance along a Lamb surface in combination with traversing normal to the Lamb surfaces without affecting the results. This allows the integration to avoid potential trouble areas such as areas requiring travel through geometry, or areas of potential discontinuity like the trailing edge of a turbofan nacelle. Additionally, it indicates that it is not only possible to integrate straight out of the streamtube normal to the tube axis, but also possible to travel along a streamline back upstream to the actuator disk. Lastly, it would also be possible to follow a streamline downstream to where the stagnation pressure has re-equilibrated with freestream stagnation pressure again. 


\section{Chapter 6}

\section{Particle Actuator Disk Validation}

Once an understanding of actuator disk theory was established it was then appropriate to determine the feasibility of a vortex particle actuator disk model. It was clear that due to the slipstream contraction of heavily loaded theory, it would be much more ideal as a basis for comparison than the lightly loaded cases. As indicated in Chapter 5, this required that the parabolic loading distribution be implemented for comparison, since the elliptic loading distribution had infinite tip gradients.

\subsection{Particle Discretization}

The particles were placed in the field using the same discretization scheme as was applied in Chapter 4 for the ring simulation. Figure 6.1 is repeated from the simulation to show the equal area scheme with which particles were again placed. The particle approximation of the actuator disk solution was time dependent, and as such, at $t=0$ an initial group of particles was placed to approximate the disk. The influence on these particles was then calculated, the particles were convected, and a 


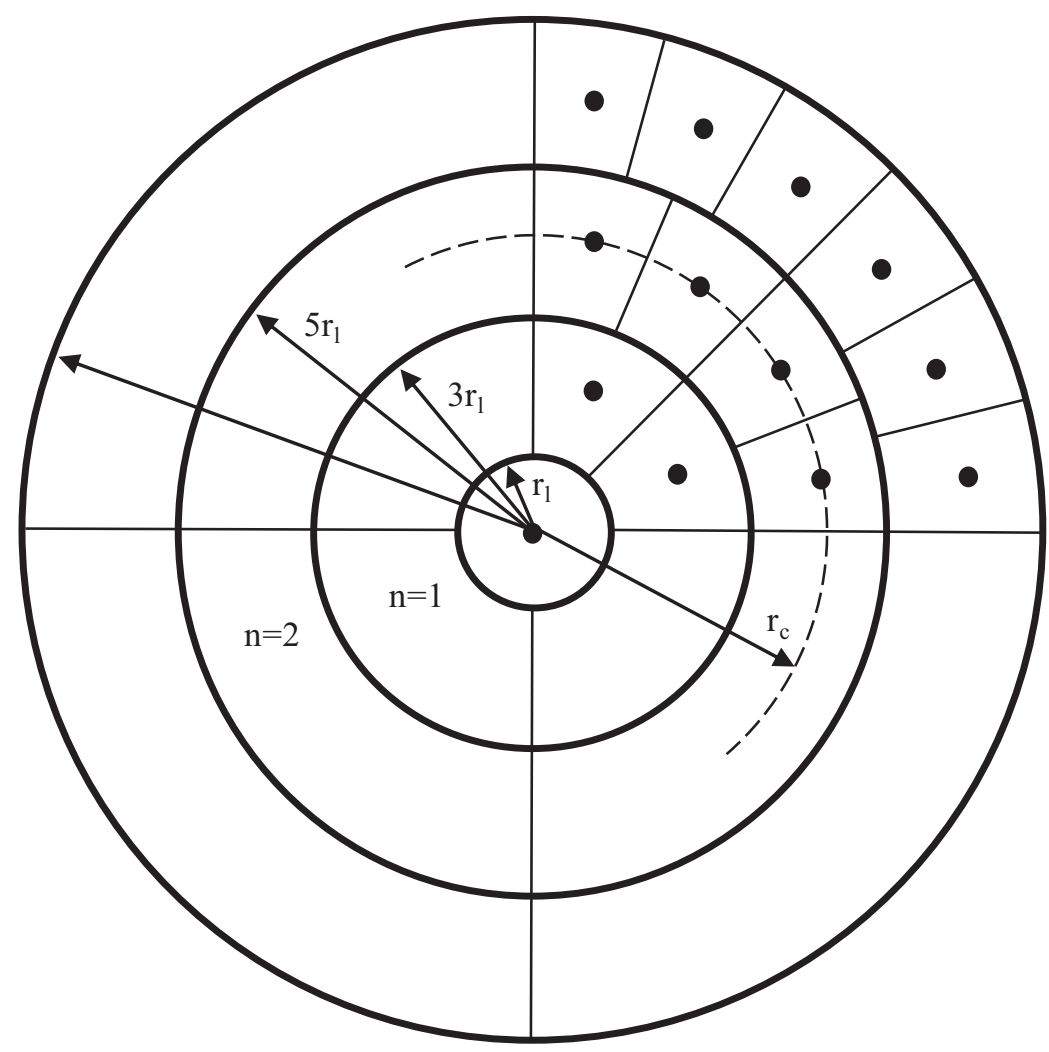

Figure 6.1: Discretization of particles into a two dimensional disk $\sqrt[3]{3}$

new group of particles was introduced for the next time step.

\subsubsection{Vortex Cores Sizing}

Once the first disk of particles was placed at $t=0$, the vortex core radius was solved for. Since the particle cells had equal area, the radial distance between particles and their neighbors in a single disk was constant. The radial $h$ value was determined based on the number of particles and disk radius specified by the user. The time step size was then determined to ensure that the axial $h$ value was equal to the radial value, thereby ensuring equal core overlap in all dimensions. This was done 
by assuming that the particles in the disk would be convected a distance of $V_{\infty}(d t)$ downstream, and therefore setting $h=V_{\infty}(d t)$. This assumption was quite reasonable for the first time step, where most of the velocity influence on the particles was from freestream. As the flow evolved and more particles were placed in the field the assumption breaks down because the velocity influence from the actuator disk particles becomes significant compared to freestream, especially at high $C_{T}$ values. The breakdown of this assumption leads to the possibility of losing vortex core overlap in the simulation, which can result in numerical instabilities and errors.

\subsubsection{Particle Strength Assignment}

If there was to be any hope of matching actuator disk theory, it was essential that the particles receive correct strength vectors that would accurately model the same flowfield as the theory. According to Conway, the only required input to solve for the strengths of the particles is an exact desired axial velocity distribution at the disk, $V_{z}(r, 0) \stackrel{48}{{ }^{4}}$ This is related to particle strengths through

$$
\frac{d V_{z}(r, 0)}{d r}=-\frac{\gamma(r)}{2}
$$

where $\gamma(r)$ is the azimuthal strength of the vortex tubes with respect to the radial location on the actuator disk.

Appendix $\mathrm{B}$ shows that the thrust coefficient is also related to the perturbation velocity distribution, so the combination of a velocity profile, along with a thrust coefficient to scale that profile, provides enough information to assign reasonable 
particle strengths.

\subsection{Time Evolution}

The same time evolution framework that was initially used in the vortex ring simulation in Subsection 3.5 was again applied to the vortex particle actuator disk in double precision. The third order Runge-Kutta solver described in Section 4.4 was used for both the position and strength evolutions. The number of time steps was automatically determined based on a user specified number of propeller radii downstream that the wake should be convected using the $V_{\infty}$ convection rule. A particle wake with roll up appears because of the unsteady nature of the solution, and an example of this is shown in Figure 6.2. The flowfield is created by instantaneously

turning the actuator disk on from rest to full strength at time zero. As such, to achieve a pseudo steady state solution the wake must be convected far enough downstream that its influence is negligible in all areas of interest in the flowfield.

To determine the number of time steps required to ensure the wake has reached this distance, the freestream velocity is again applied to determine the amount of time to convect particles released at time zero the specified distance. The total time is then divided by the time step size determined in vortex core sizing to determine the number of time steps required. This approximation may initially appear conservative, as the velocity at which particles are convected can be much greater than freestream in the core of the slipstream, depending on whether lightly or heavily loaded theory is 


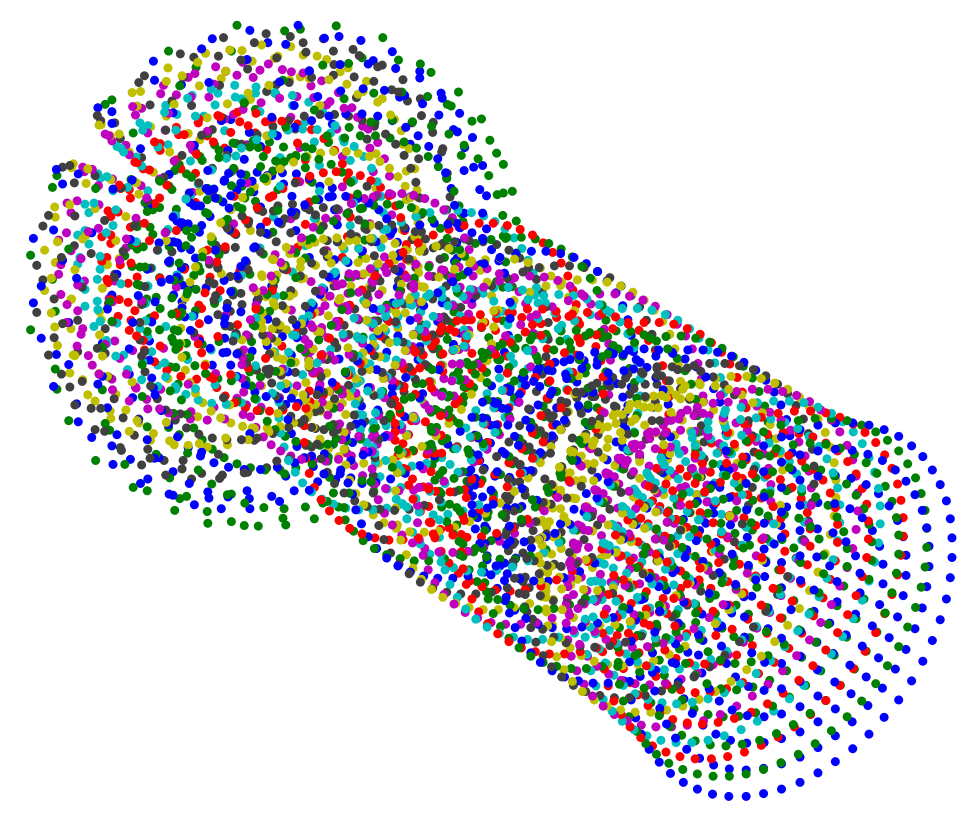

Figure 6.2: A sample particle streamtube with unsteady wake roll up occurring.

applied. On the boundaries of the slipstream however, the velocities tend to remain close to freestream. Additionally, the wake roll up effect means that the last several groups of particles appearing in the disk end up with total velocities directed upstream toward the end of the simulation.

\subsection{Simulation Results}

Once the particle solution had been completed through the introduction of a new group of particles and the convection of all particles for each time step, the results were analyzed and compared. The loading distribution examined in comparison with Conway's work was the parabolic case. Both the axial and radial velocities are compared with actuator disk theory at a variety of axial locations. Additionally, contours 
of vorticity are examined as a technique of determining smoothness of the solution due to vortex particle discretization.

A great deal of variation arises through different matching techniques, and it is not clear which results are the most ideal for use in comparison with experimental data. As such, the results for matching various characteristics of actuator disk theory will be presented independently. In addition to the different possible methods for matching actuator disk theory, the number of particles used to discretize the disk affects the results.

Increasing the number of particles used makes discretization more accurate, because the continuous vorticity distribution of theory is simulated by an increasing number of points. Due to the interrelated nature of the particle count with time step size and number of times steps, a change in particle count has many trickle down effects. In addition to the computational cost associated with an increase in the number of bodies being used, the higher particle count means smaller radial distances between particles, which leads to smaller core sizes, and therefore smaller time step sizes. To achieve the same convection distance downstream additional time steps are now required beyond the lower particle solutions, adding yet another source of computational cost increase.

Without the advantage of many of the computational methods currently employed in $N$-body problems this difference can be staggering. 1] 5,6$] 11,13,55,60$ When simulating an actuator disk with $n=225$ around 20 time steps were used to reach a 3 radii 
convection distance with only a few minutes of computational time, while at $n=$ 1,521, 59 time steps were required and almost 48 hours of computation.

\subsubsection{Matching $\hat{a}$}

The most straightforward method for attempting to match the particle scheme of Conway is to assign strengths based on the $\hat{a}$ term of Equation 5.24. Conway creates plots for the parabolic loading case for $\hat{a}=5$, so this value is explicitly defined in the code. From the defined $\hat{a}$, the $a$ value is found through the relationship of Equation 5.24. Because in the parabolic loading case the variation of enthalpy within the slipstream is constant, the azimuthal vorticity is simply a function of the radius, written as

$$
\omega_{\phi}=a r .
$$

The relationship of Equation 6.2 shows that a linear variation of vorticity should be expected when traversing from one propeller tip across the disk to the opposite tip. In these sample cases $\hat{a}=5$, while $R_{a}=1$ and $U_{\infty}=1$, meaning that $a=\hat{a}=5$ through Equation 5.24. Plugging the defined value of $a$ into Equation 6.2 shows that theory expects a vorticity of $\omega_{\phi}=5$ at one tip, -5 at the opposite tip, and 0 at the disk hub.

The vorticity from the regularized vortex particles can be calculated using

$$
\boldsymbol{\omega}_{\sigma}(\mathbf{x}, t)=\sum_{p} \boldsymbol{\alpha}^{p}(t) \zeta_{\sigma}\left(\mathbf{x}-\mathbf{x}^{p}(t)\right),
$$

and so it is possible to find the vorticity at any point in the field as the sum of 
the influence from all particles in that field. Figure 6.3 shows the vorticity over the diameter of the actuator disk for the vortex particle scheme. Because of the

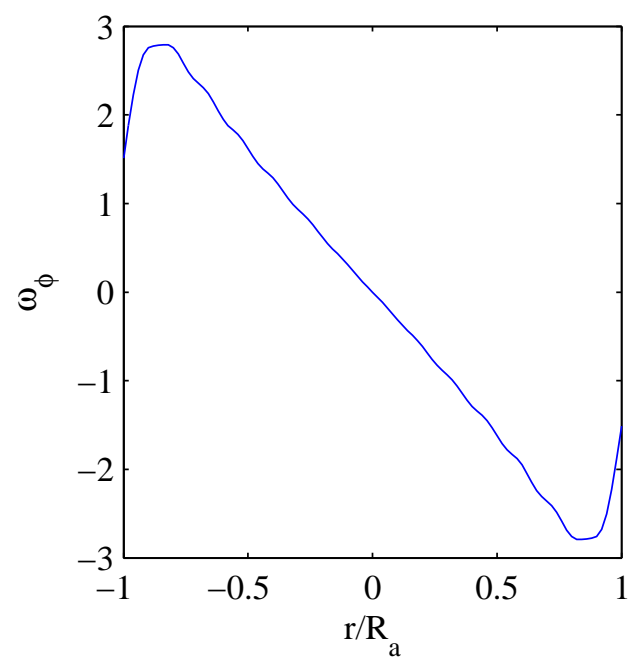

(a) $n=225$

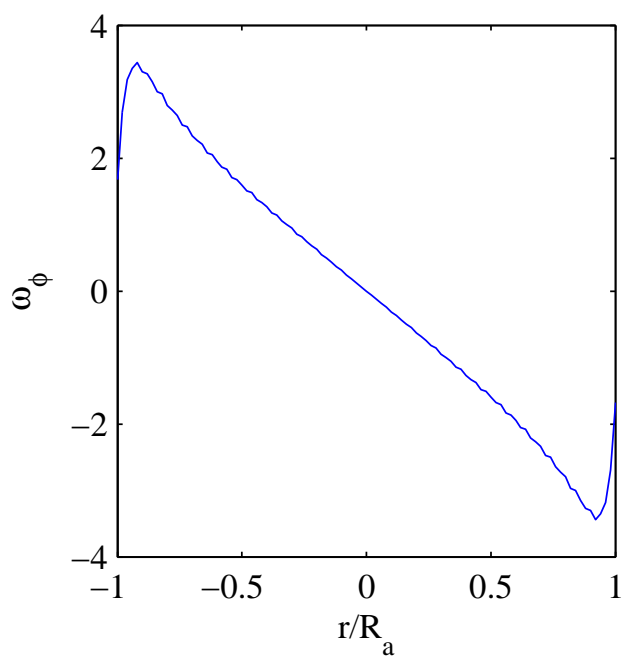

(b) $n=1,521$

Figure 6.3: Vorticity across the diameter of the actuator disk for (a) $n=225$ particles per disk and (b) $n=1,521$ particles per disk.

axisymmetry of the problem, the $y$ and $z$ values for the start point of the survey line don't matter, so long as the survey line across the disk splits it into two even halves. When the particle count is 225 the theoretical linear trend is visible in the approximation. The magnitude of the vorticity everywhere however, is reduced from what theory predicts, with the peak vorticity at the disk tips only reaching about half of the theoretical value of 5 . When the particle count was increased to $n=1,521$ the presence of a larger number of particles was visible in the vorticity distribution across the disk. The number of ripples has greatly increased due to the increased number of particles discretizing the disk.

In a similar manner to examining the vorticity across the actuator disk, it is also 
possible to survey the vorticity inside the streamtube behind the disk. The vorticity is still sampled using Equation 6.3, this time over a grid constructed in the streamtube. Figure 6.4 displays contours of vorticity throughout the survey area of the streamtube. A small number of particles results in larger time steps to maintain the required

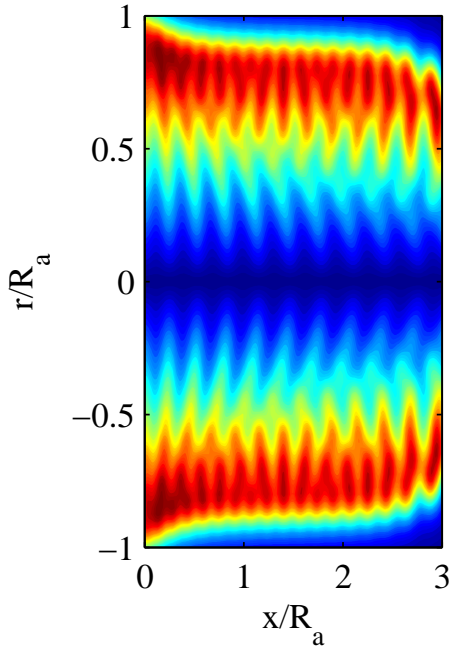

(a) $n=225$
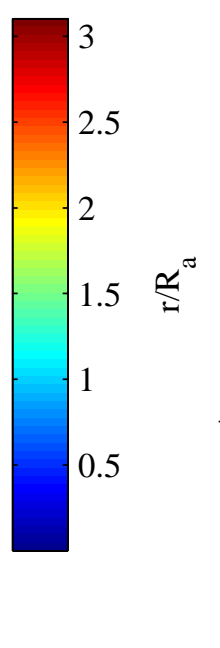

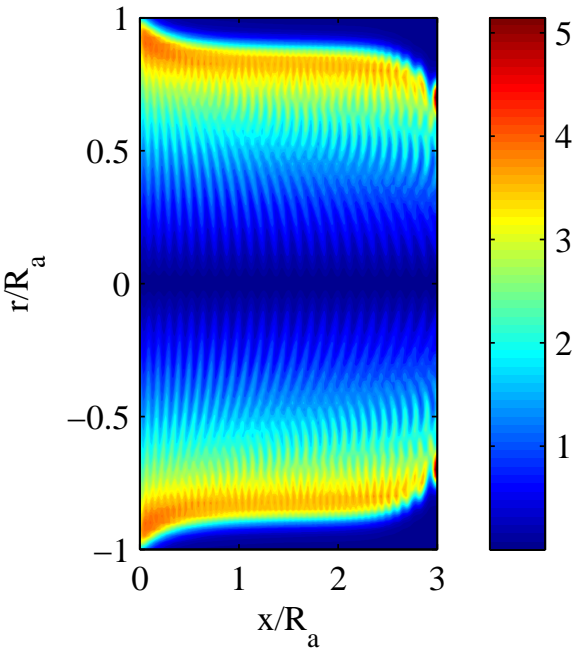

(b) $n=1,521$

Figure 6.4: Vorticity inside the streamtube downstream of the actuator disk for (a) $n=225$ particles per disk and (b) $n=1,521$ particles per disk.

vortex core overlap. As a result the contours of vorticity are not smooth through the streamtube for $n=225$. When the particle count is increased to $n=1,521$ the vorticity field becomes much smoother. The field doesn't become completely smooth though, because the vortex cores shrink with the increased particle count. The result is smaller amplitude, but higher frequency oscillations in the vorticity contours. This refinement seems to indicate that in the infinite limit of particles per disk, the vorticity field would become completely smooth.

A pseudo-steady vorticity field provides the information necessary to obtain veloc- 
ity information in the steady areas of the streamtube. Figure 6.5 shows a comparison of the exact axial velocity distributions of Conway using solid lines and the approximate solution from the vortex particle scheme using dashed lines for two densities of particles. Clearly, when using 225 particles the matching is quite good upstream

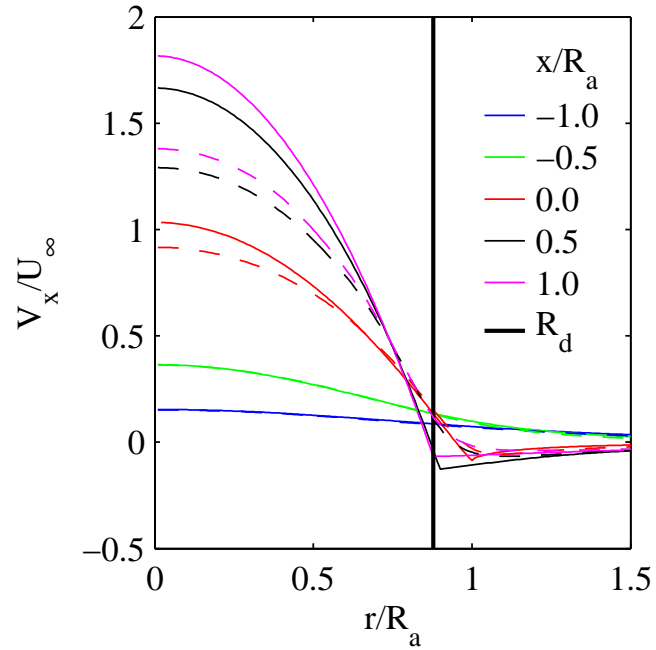

(a) $n=225$

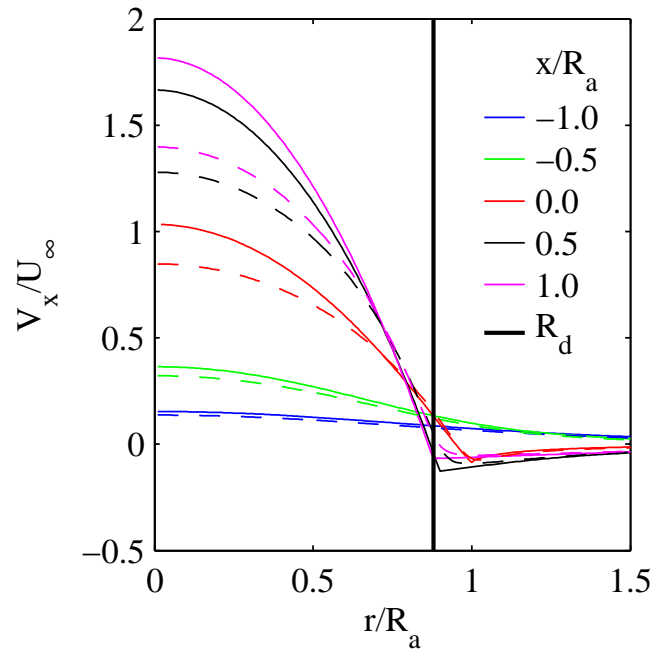

(b) $n=1,521$

Figure 6.5: Comparison of analytical (solid) and discretized vortex particle (dashed) axial perturbation velocities at several axial positions relative to the actuator disk for (a) $n=225$ particles per disk and (b) $n=1,521$ particles per disk.

of the disk, but begins to break down at the disk. The error at the disk between theory and approximation is largest at the root, while the section from the tip to the mid section of the blade still matches well. The axial section with the largest error is downstream of the disk, where the velocity magnitudes are underpredicted. Increasing the particle count from $n=225$ to $n=1,521$ while still keeping $\hat{a}=5$ has minimal downstream effects, but does cause slight reductions in velocity magnitude at the disk and upstream of it. Perhaps the most subtle difference, but also the most 
important, is that the increased particle count results in much better matching of the discontinuity at the streamtube border than was present when $n=225$.

The radial perturbation velocity can be examined at various axial stations in a similar way to the axial velocity, and is shown in Figure 6.6. Obviously the matching

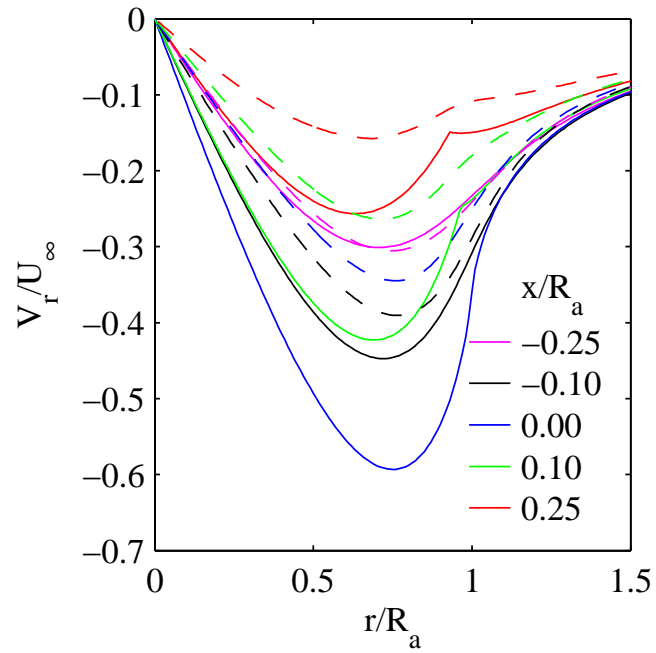

(a) $n=225$

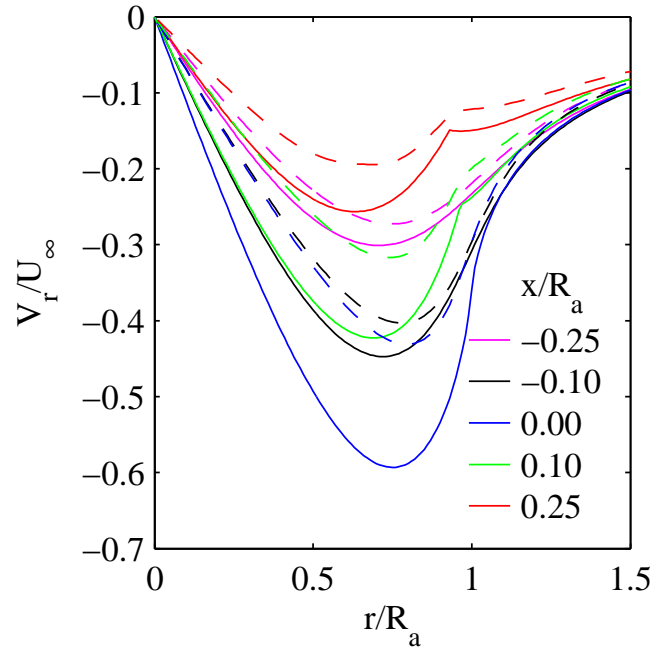

(b) $n=1,521$

Figure 6.6: Comparison of analytical (solid) and discretized vortex particle (dashed) radial perturbation velocities at several axial positions relative to the actuator disk for (a) $n=225$ particles per disk and (b) $n=1,521$ particles per disk.

for the radial velocity is not nearly as good as for the axial velocity. The radial velocity appears to be a higher order problem, with lower particle counts providing drastically worse approximations to the true solution than might be expected from the same particle count in the lower order axial perturbation velocities. Even though matching between theory and approximation is not great, the trends are still captured well, with smoothed versions of the streamtube discontinuity still appearing at the downstream locations. Similar to the axial case, the upstream velocities more closely 
match theory than do the velocities at or downstream of the disk. When the particle count per disk is increased to $n=1,521$ the matching improves for all axial locations except far upstream from the disk. Much like the axial velocity case, the higher particle count does a better job of capturing the discontinuity at the edge of the streamtube.

One final examination of the simulation is a comparison of the streamtubes approximated by each particle count. Figure 6.7 shows the evolved streamtubes for each particle discretization count. The shape of the streamtubes for each particle count

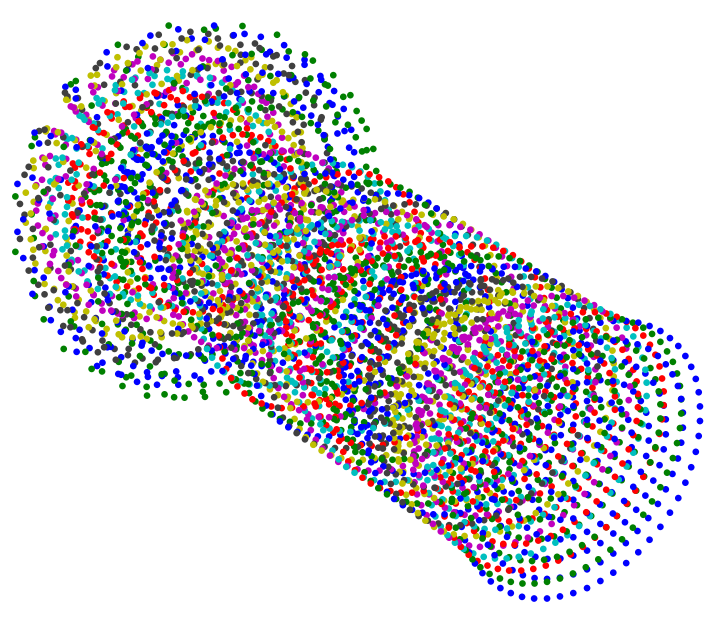

(a) $n=225$

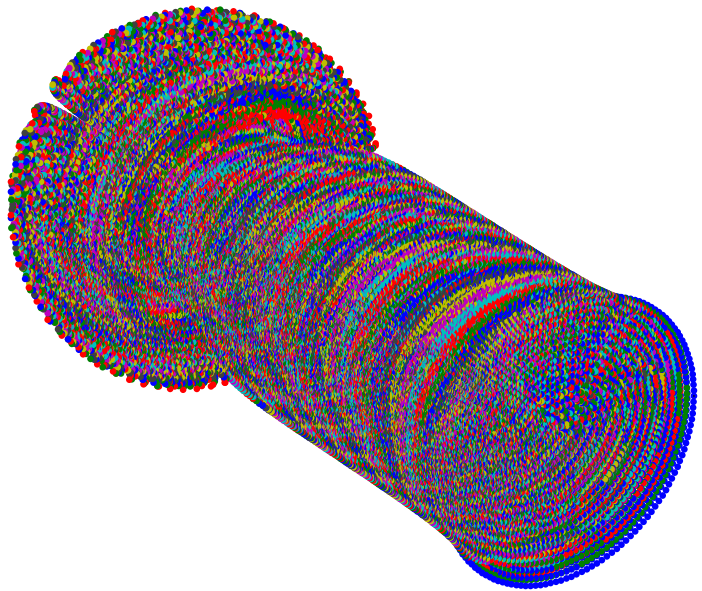

(b) $n=1,521$

Figure 6.7: Final particle locations after convection for (a) $n=225$ particles per disk and (b) $n=1,521$ particles per disk.

appear qualitatively identical, but the higher particle count case has a much more clearly defined streamtube at the current resolution than its lower particle counterpart. 


\subsubsection{Matching Axial Velocity at the Disk}

With a little input and tuning it is possible to set particle strengths to closely match theoretical perturbation velocity distributions at any of the axial locations of interest. This section will look specifically at matching the perturbation velocity profile at the disk location, where $x / R_{a}=0$. The $\hat{a}$ is adjusted until the least square error at the disk is approximately minimized, which corresponds to $\hat{a}=5.55$ rather than 5 as in the theoretical case. When the particle count is increased, further adjustment is required to get the best match for velocity at the disk. The new $\hat{a}=5.87$ carries some interesting results.

Because the $\hat{a}$ value was adjusted to match the velocity at the disk, which was previously underestimated, the vorticity values assigned to the particles through Equation 6.2 are increased. This results in a slightly increased linear distribution from the one seen in Figure 6.3, although the values still fall short of the magnitudes expected from theory, again likely due to regularization and discretization. Figure 6.8 shows the new vorticity across the disk. The addition of extra particles in the discretization has the same results in this case as it did when matching the $\hat{a}$ value of Conway. The smaller core radius used in the higher particle count causes less washout of vorticity, so the peak in vorticity is less smooth, and happens closer to the tip of the disk than with the lower particle count. The larger particle number is again reflected in the higher number of ripples across the radius of the disk.

In addition to the vorticity across the disk, the vorticity in the streamtube is also 


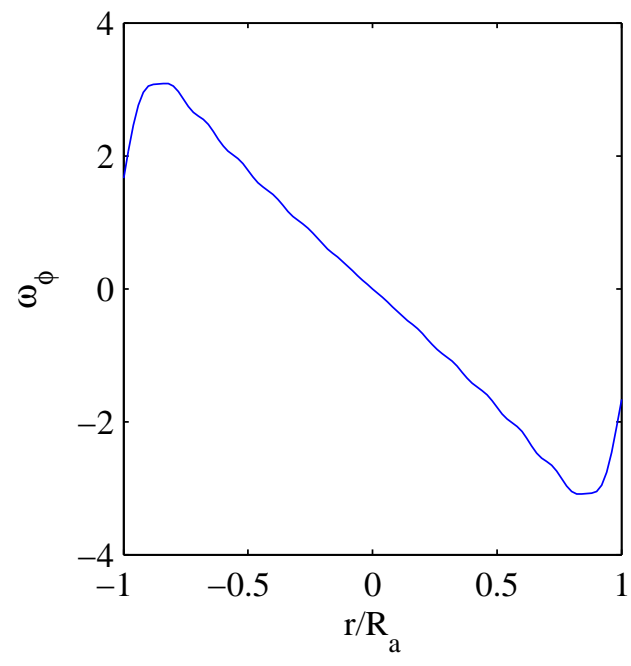

(a) $n=225$

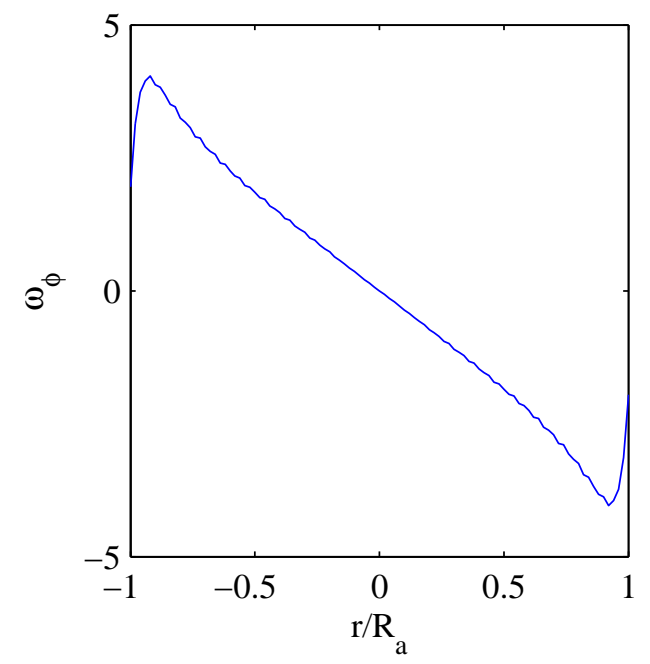

(b) $n=1,521$

Figure 6.8: Vorticity across the diameter of the actuator disk for (a) $n=225$ particles per disk and (b) $n=1,521$ particles per disk.

revisited in Figure 6.9. The same trends witnessed previously are again seen. The small particle count leads to large time steps and therefore a non-smooth vorticity field throughout the streamtube. Magnitudes are slightly different due to the adjusted $\hat{a}$, but the overall trends are identical. The higher particle count again causes smaller, higher frequency oscillations in the vorticity contours than were present in the low particle case. The larger vorticity magnitudes are reflective of the higher values seen in Figure 6.8, but the trends between the high and low particle counts are the same.

Figure 6.10 shows the comparison of theoretical and approximate axial velocity profiles. When $n=225$ the shape of the profile inside the streamtube is not quite the same as in theory, with the gradient being slightly more gentle in the approximate case. When $n=1,521$ the matching at the disk isn't quite as good as with the lower particle count because the shape of the distribution has changed slightly. At 


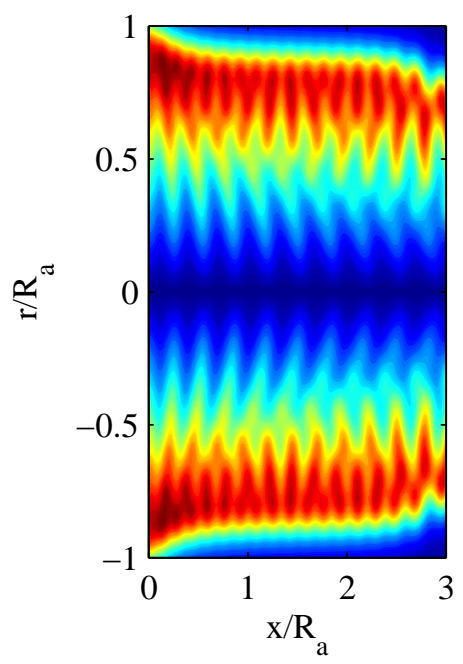

(a) $n=225$

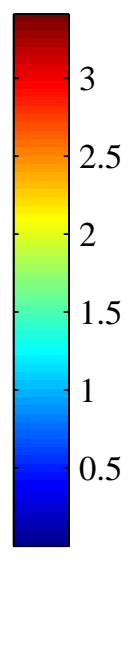

$\stackrel{2}{=}$

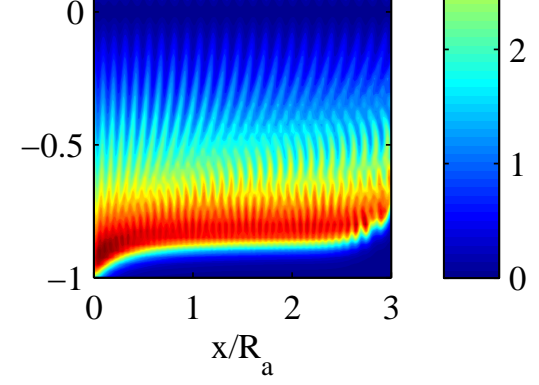

(b) $n=1,521$

Figure 6.9: Vorticity inside the streamtube downstream of the actuator disk for (a) $n=225$ particles per disk and (b) $n=1,521$ particles per disk.

all other axial locations however, the matching has improved. Matching upstream of the disk is nearly perfect, while downstream the magnitudes have increased closer to the analytical values. Additionally, the discontinuities at the streamtube boundary are once again captured much better with the higher particle count.

The radial velocity distributions are presented in Figure 6.11. The radial perturbation velocities match approximately as well as they did when the theoretical $\hat{a}$ was matched. Much like in the prior case where $\hat{a}$ was matched, here when a higher number of particles is used the radial velocity matching greatly improves. In this case the accuracy at every axial location has improved due to the addition of particles. Once again the discontinuities at the streamtube boundary are better captured with the higher particle count. 


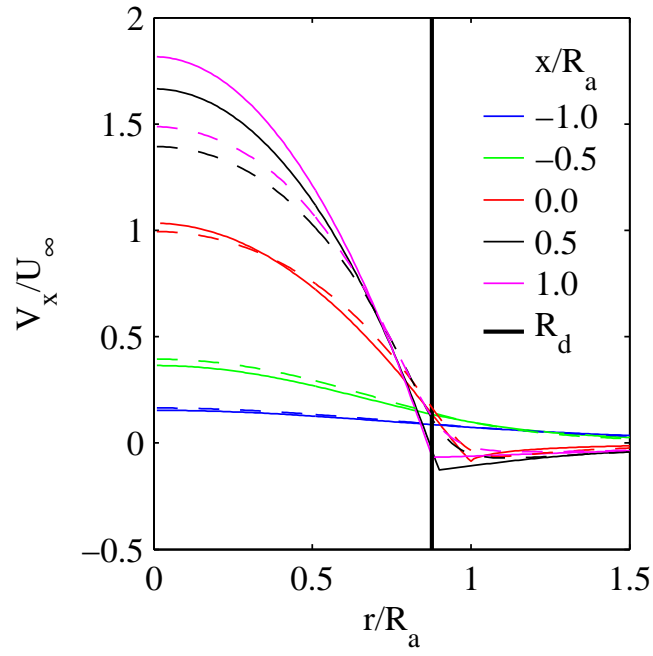

(a) $n=225$

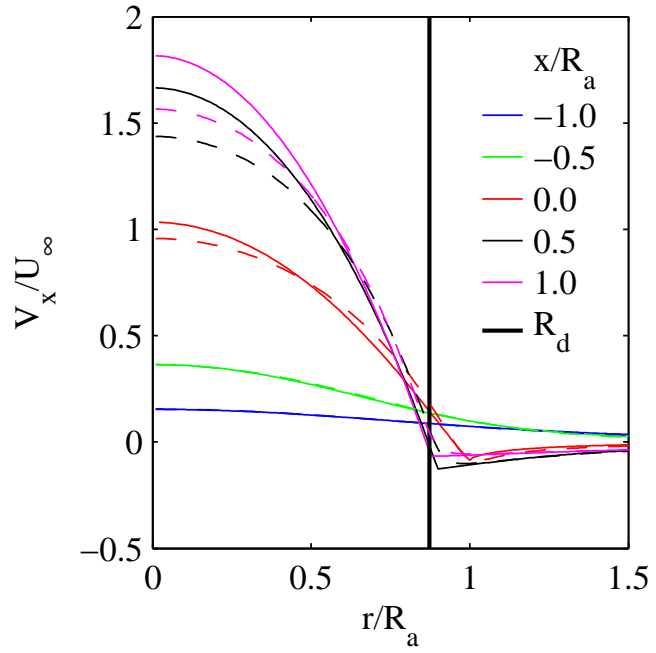

(b) $n=1,521$

Figure 6.10: Comparison of analytical (solid) and discretized vortex particle (dashed) axial perturbation velocities at several axial positions relative to the actuator disk for (a) $n=225$ particles per disk and (b) $n=1,521$ particles per disk.

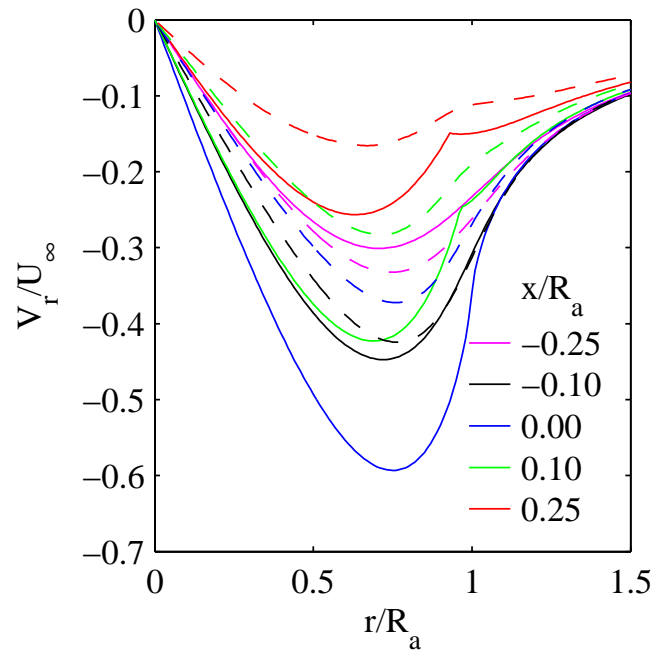

(a) $n=225$

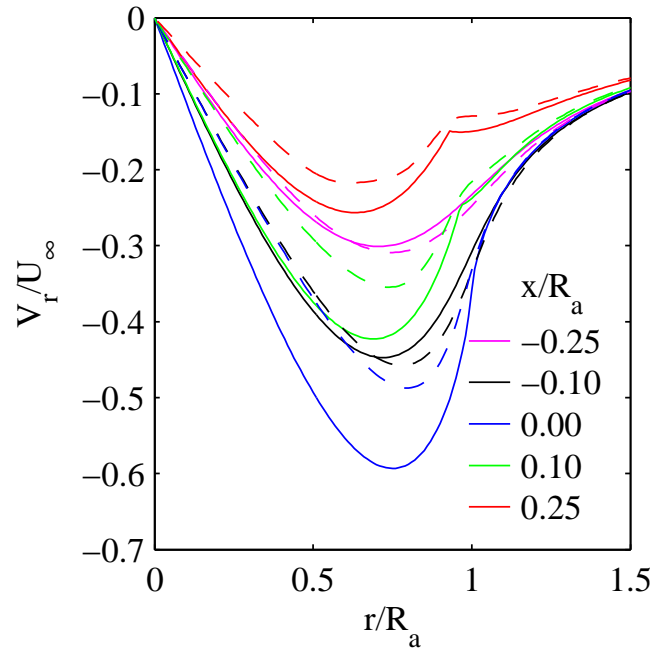

(b) $n=1,521$

Figure 6.11: Comparison of analytical (solid) and discretized vortex particle (dashed) radial perturbation velocities at several axial positions relative to the actuator disk for (a) $n=225$ particles per disk and (b) $n=1,521$ particles per disk. 


\subsubsection{Matching Vorticity Distribution Slope}

Rather than matching the theoretical $\hat{a}$ value, it may be more appropriate to match the vorticity that is a product of that value. As discussed previously, the vorticity distribution at the disk should be linear, with zero vorticity at the disk center and maximum vorticity at the propeller tips. The $\hat{a}$ value defined in the particle approximation can be adjusted in a similar manner to the previous example, with the objective in this case being to match the overall slope of the vorticity distribution at the disk. The magnitude of the vorticity at the tip for the case in question should be 5 , and with a propeller radius of 1 , the slope must be 5 . Because the approximate vorticity distribution is not completely linear, as a simple test the $\hat{a}$ value was adjusted until the strength at $r=0.5$ was about 2.5 . To achieve the correct slope the $\hat{a}$ value was increased to 8.0 for $n=225$. The simple approximation of slope based on vorticity at $r=0.5$ was not quite as useful when $n=1,521$. This is because the slope line begins to acquire curvature. For this case the slope of the best fit line was instead set to 5 .

Figure 6.12 shows the vorticity across the actuator disk. Clearly the figure demonstrates that the slope of vorticity does indeed match the theoretical slope of 5 since at $r=0.5$ the vorticity is $\omega_{\phi}=2.5$. The vorticity distribution for the higher particle count is quite similar to that of the distribution when $n=225$, however there are some subtle differences. The higher particle count is slightly less linear than its lower particle counterpart, but clearly the average slopes are approximately identical. 


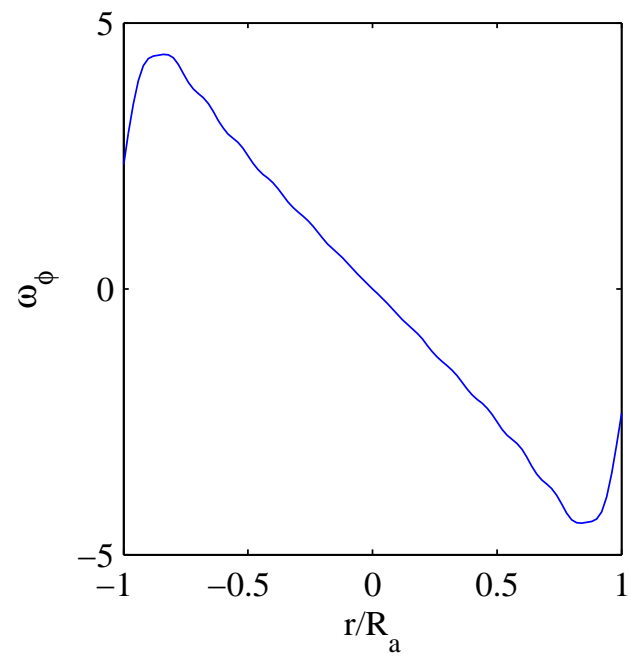

(a) $n=225$

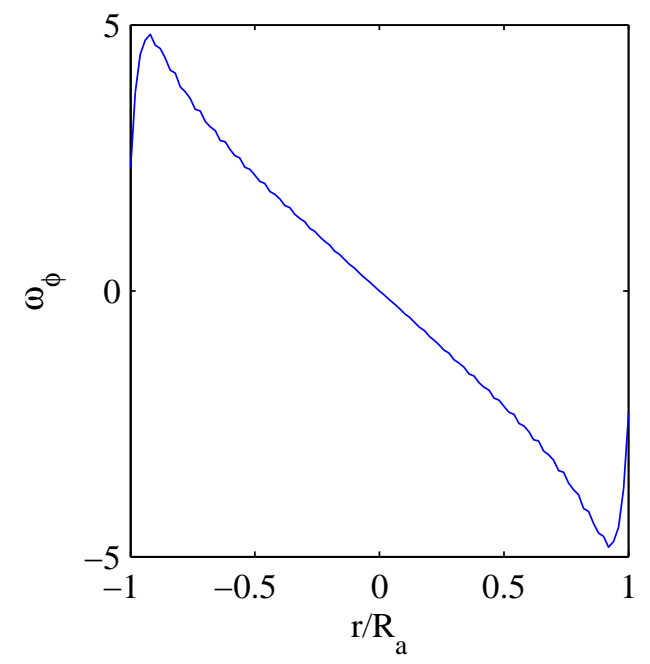

(b) $n=1,521$

Figure 6.12: Vorticity across the diameter of the actuator disk for (a) $n=225$ particles per disk and (b) $n=1,521$ particles per disk.

Much like in previous cases, the increase in particle count results in smaller, more high frequency ripples in the vorticity distribution.

The vorticity in the streamtube is examined in 6.13. Clearly the trends remain the same as the previous two cases, although the magnitudes of the contours were again increased due to the increase in $\hat{a}$. Increasing the particle count used to discretize the disk still results in smaller, higher frequency fluctuations in the vorticity throughout the streamtube.

The axial velocity profiles at various axial locations are shown again in Figure6.14. The matching is poor for all axial stations when $n=225$. This is the first of the examined matching cases where the downstream axial perturbation velocities are over-predicted. Increasing the particle count to $n=1,521$ provides better matching at all five axial locations. The upstream and at-disk velocity profiles match much 


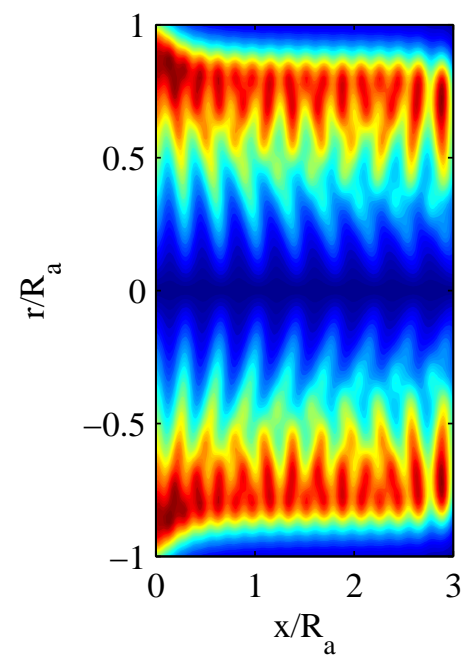

(a) $n=225$
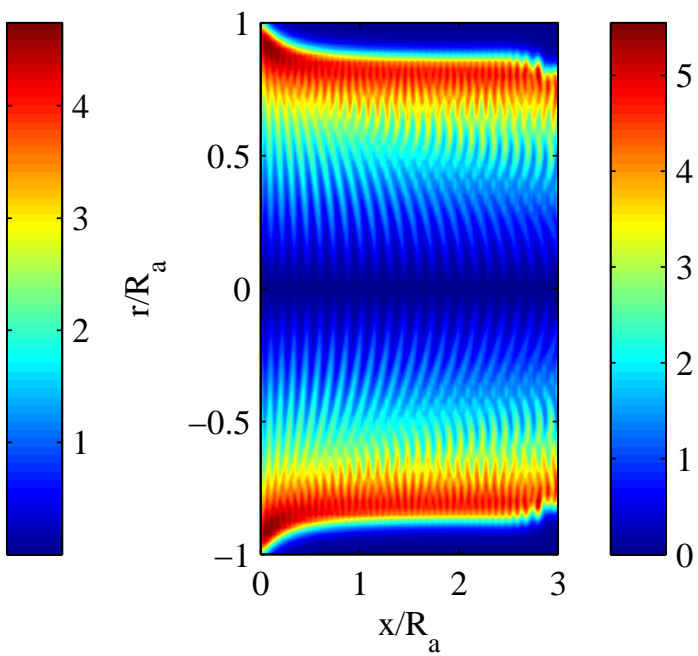

(b) $n=1,521$

Figure 6.13: Vorticity inside the streamtube downstream of the actuator disk for (a) $n=225$ particles per disk and (b) $n=1,521$ particles per disk.

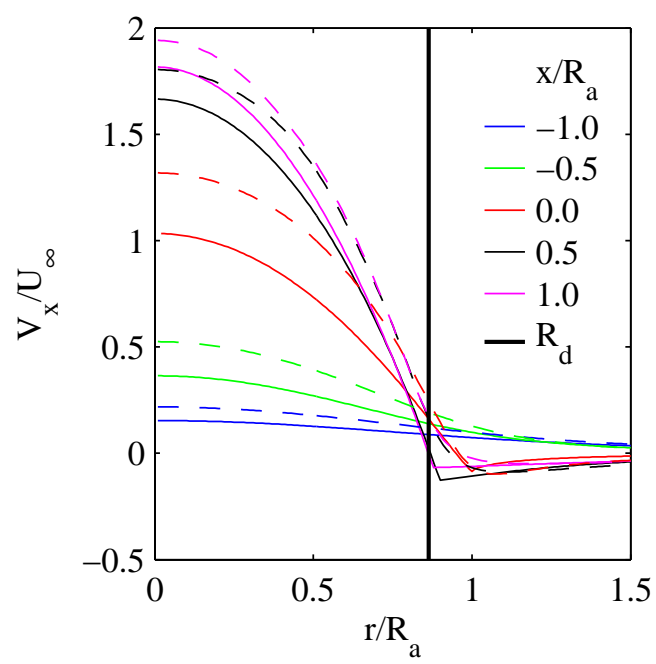

(a) $n=225$

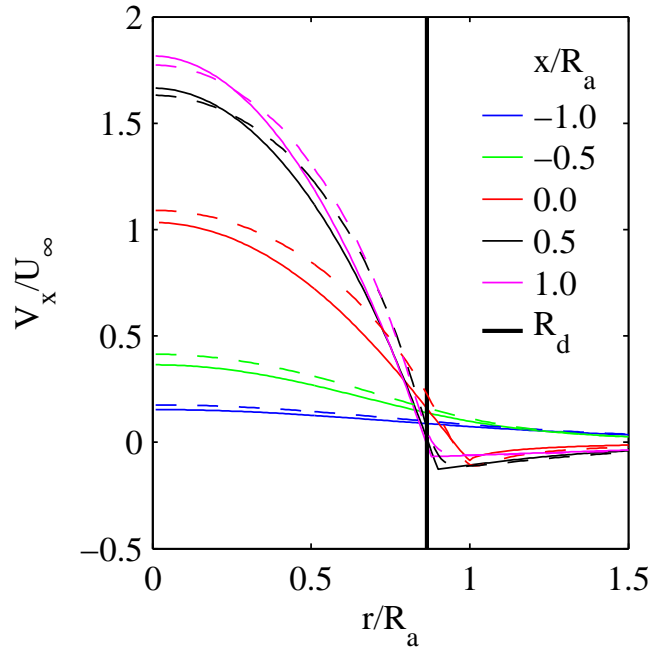

(b) $n=1,521$

Figure 6.14: Comparison of analytical (solid) and discretized vortex particle (dashed) axial perturbation velocities at several axial positions relative to the actuator disk for (a) $n=225$ particles per disk and (b) $n=1,521$ particles per disk. 
better than when $n=225$, but the greatest improvement is in the downstream matching. Both the accuracy of contraction of the downstream streamtube radius and the maximum magnitude of perturbation velocity are nearly exact.

The radial perturbation velocities are also reexamined in Figure 6.15, with interesting results. The matching continues to be lower quality for radial velocity than for

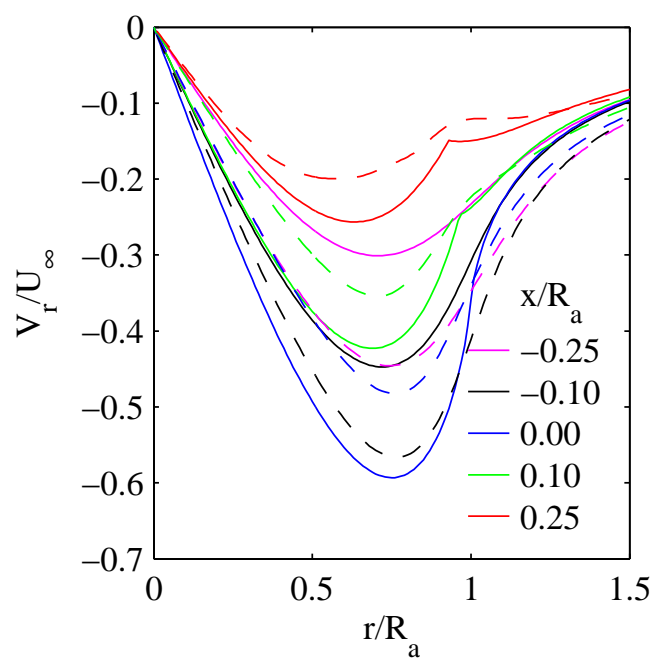

(a) $n=225$

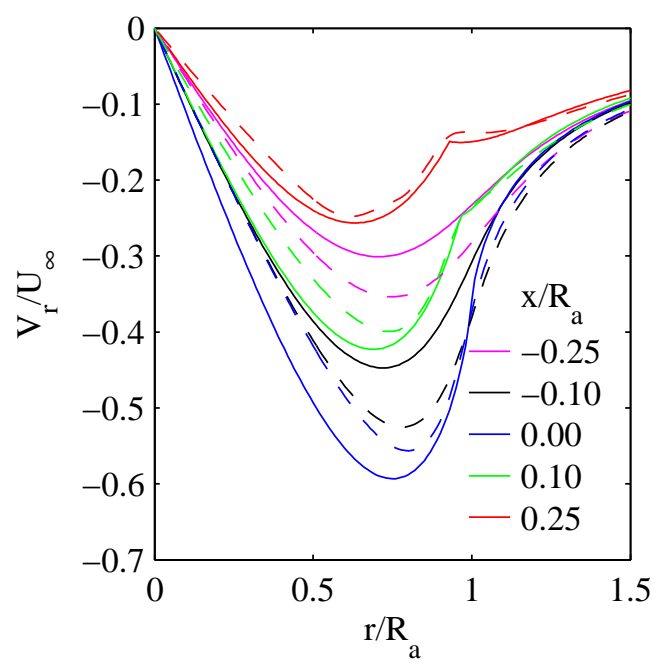

(b) $n=1,521$

Figure 6.15: Comparison of analytical (solid) and discretized vortex particle (dashed) radial perturbation velocities at several axial positions relative to the actuator disk for (a) $n=225$ particles per disk and (b) $n=1,521$ particles per disk.

axial velocity. In previous cases however, the matching was only somewhat valuable for upstream axial locations. When switching to matching vorticity slope however, the matching is much closer for the downstream locations. Again, the increase in particles to $n=1,521$ results in better matching at all axial locations. The downstream streamtube boundary discontinuity is much more clearly defined. In addition to the better matching of the discontinuity, the matching of magnitudes of velocity profiles 
is also much improved, particularly at, and downstream of the disk.

\subsubsection{Matching Maximum Vorticity}

In a similar manner to the previous section, an aspect of the vorticity in the flowfield can again be matched to. In this case though, the maximum vorticity, rather than the slope of the vorticity distribution, is matched to theory. Correspondingly, the $\hat{a}$ is adjusted until the maximum value of the approximated vorticity distribution is equal to the theoretical maximum of 5 . The $\hat{a}$ value that matches theoretical maximum vorticity is $\hat{a}=9$ when $n=225$. When the particle count is increased to $n=1,521$ the $\hat{a}=7.2$.

Figure 6.16 shows the vorticity distributions across the disk while matching the maximum vorticity. The figure shows that for each particle count the $\hat{a}$ value was

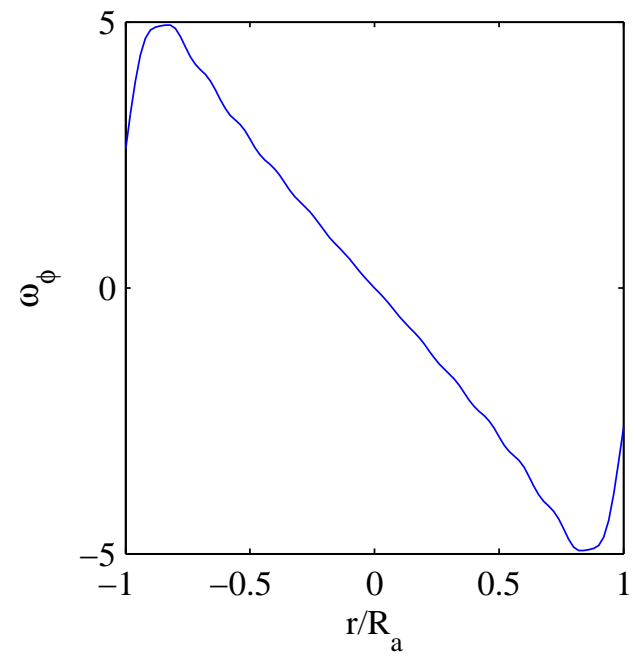

(a) $n=225$

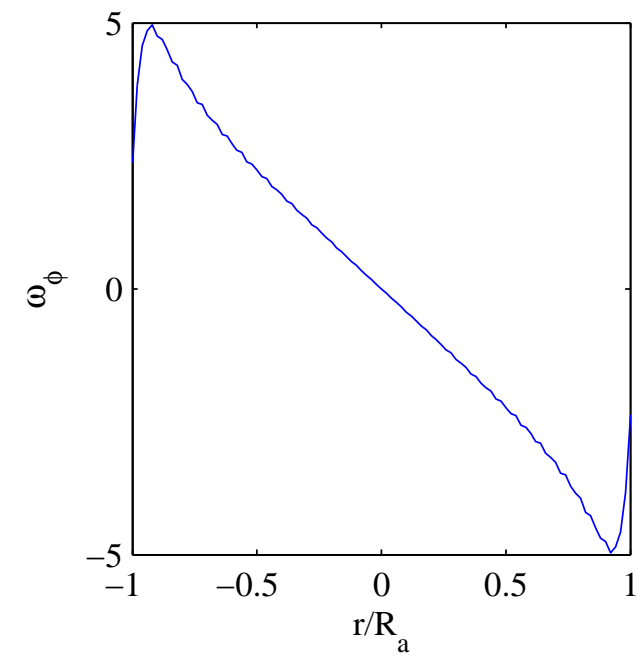

(b) $n=1,521$

Figure 6.16: Vorticity across the diameter of the actuator disk for (a) $n=225$ particles per disk and (b) $n=1,521$ particles per disk. 
adjusted to ensure that the maximum vorticity on the disk matched the theoretical maximum of 5 . It is also apparent from this figure why the solutions between matching slope and matching maximum vorticity are so similar. An examination of the slope for the two different particle counts provides visual evidence that the slope is quite close to the theoretical 5 for the high particle count. As in the other cases, the increased number of particles results in smaller amplitude and higher frequency ripples in the vorticity distribution, with some slight curvature appearing relative to the more linear distribution.

The contours of vorticity in the streamtube when maximum vorticity is matched are shown in Figure 6.17. The difference in magnitudes of vorticity in the field are not particularly large between the two cases. The higher particle count does have a distinctly smoother vorticity field than the lower particle counterpart, due to the higher number of particles with smaller core radii. As with all other figures for the maximum vorticity matching case, the solution at $n=1,521$ is extremely similar to the slope matched case because the distributions have become nearly identical.

The axial velocity profiles at various axial locations are shown in Figure 6.18 . Clearly matching the peak value of vorticity does not provide a good approximation of actuator disk theory for small particle counts. None of the matching is particularly good, but the values at the disk are even further off than the rest. A great deal of improvement is obtained by increasing the number of particles discretizing the actuator disk. When $n=1,521$ the matching upstream of the disk becomes much 


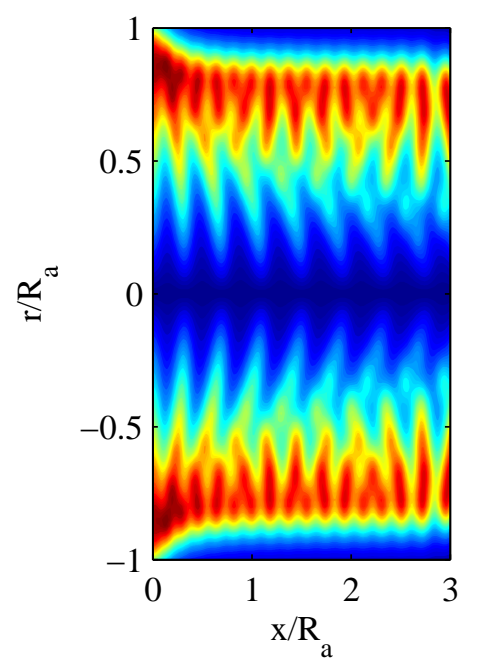

(a) $n=225$

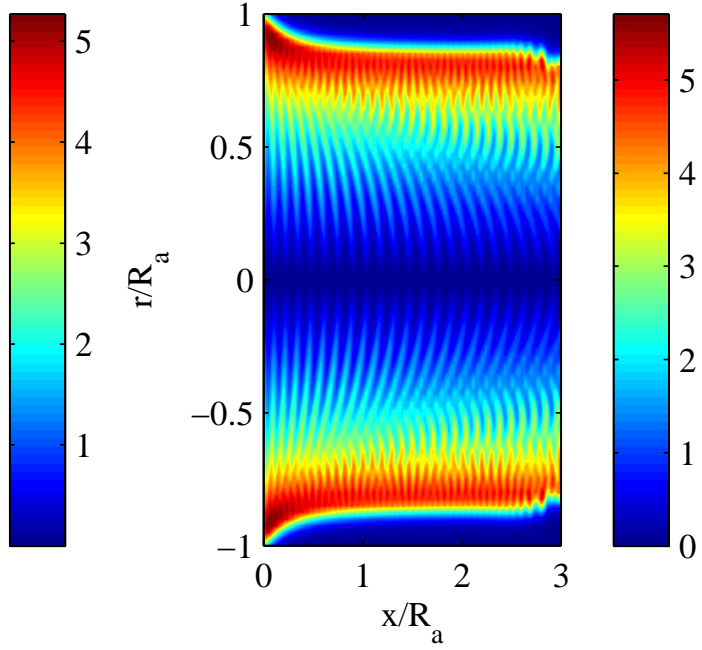

(b) $n=1,521$

Figure 6.17: Vorticity inside the streamtube downstream of the actuator disk for (a) $n=225$ particles per disk and (b) $n=1,521$ particles per disk.

more comparable to matching the vorticity slope. The velocity profile at the disk is still overpredicted, but by a much smaller margin due to the better discretization. The greatest improvement occurs downstream of the disk, where the maximum magnitudes of perturbation velocity match almost exactly. In addition, as with all other cases where the particle count was increased, the profiles capture the contracted streamtube much better when $n=1,521$.

Figure 6.19 shows the radial perturbation velocities for the different particle counts. Once again, the values for $n=225$ are poor, with the best matching coming downstream. Similar to the axial velocity distributions, the increase in particles to $n=1,521$ yields much better results, with improvement at each of the axial stations examined. The upstream profiles have decreased to values much closer to theory, while the downstream values are again almost exactly matching theory, and capturing the 


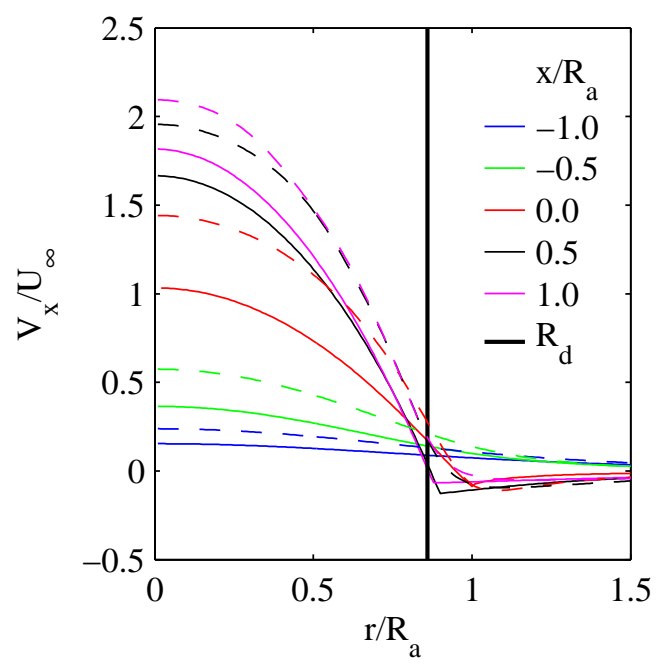

(a) $n=225$

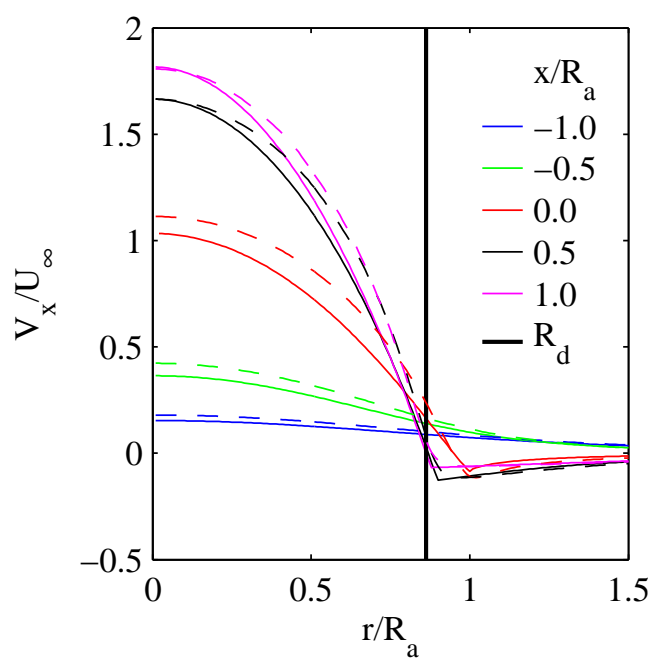

(b) $n=1,521$

Figure 6.18: Comparison of analytical (solid) and discretized vortex particle (dashed) axial perturbation velocities at several axial positions relative to the actuator disk for (a) $n=225$ particles per disk and (b) $n=1,521$ particles per disk.

streamtube discontinuity extremely well.

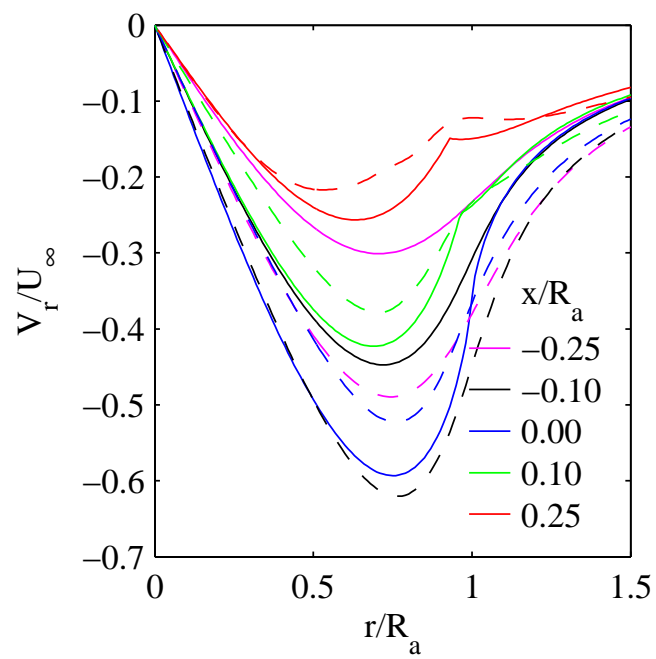

(a) $n=225$

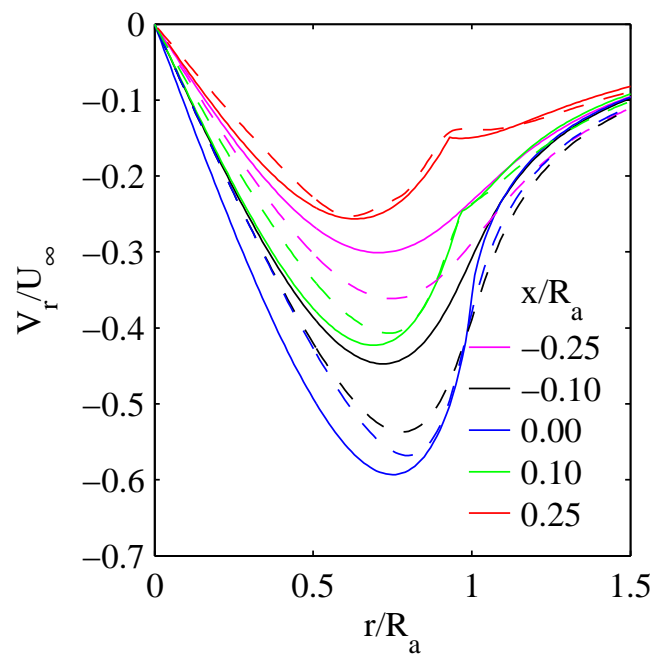

(b) $n=1,521$

Figure 6.19: Comparison of analytical (solid) and discretized vortex particle (dashed) radial perturbation velocities at several axial positions relative to the actuator disk for (a) $n=225$ particles per disk and (b) $n=1,521$ particles per disk. 


\subsubsection{Conclusions from Different Matching Schemes}

The results of each of the different matching techniques leads to the conclusion that vorticity is a fundamental component of the flowfield. This conclusion is supported by the fact that in vortex schemes, the velocity throughout the field is calculated based on the vorticity field. As such, matching vorticity provides the best solutions as the particle count is increased. For low particle counts, such as when $n=225$, matching vorticity does not do as strong of a job of modeling the vorticity field, making it is advisable to match based on desired results like perturbation velocities, rather than flowfield metrics like vorticity. For the later parts of the project it would be ideal to match to known vorticity in the field for the reasons just mentioned. Computational costs become even more prohibitive with the addition of panels however, which makes matching vorticity in interaction simulations less useful. Additionally, the conceptual designer can much more easily input an approximate velocity distribution at the disk than they could an explicit vorticity distribution in the flowfield. These factors lead to the use of perturbation velocity matching in the studies conducted for this work.

\subsubsection{Smoothed Streamtube Vorticity}

In any of the cases above, the vorticity field can be smoothed out further by decreasing the time step size, and thereby decreasing axial spacing between particles as they are convected through the field. Only the $n=225$ case will be examined for this section due to the prohibitive cost of doubling and quadrupling the number of 
time steps being carried out at higher particle counts. The increased number of time steps results in a higher number of particles in the field over the same convection distance. This means that to obtain axial velocity results at the disk similar to the cases run previously the $\hat{a}$ must be reduced to compensate.

Figure 6.20 shows the vorticity survey at the disk for $n=225$ when the time step is halved as well as when it is quartered. The decrease in time step size results

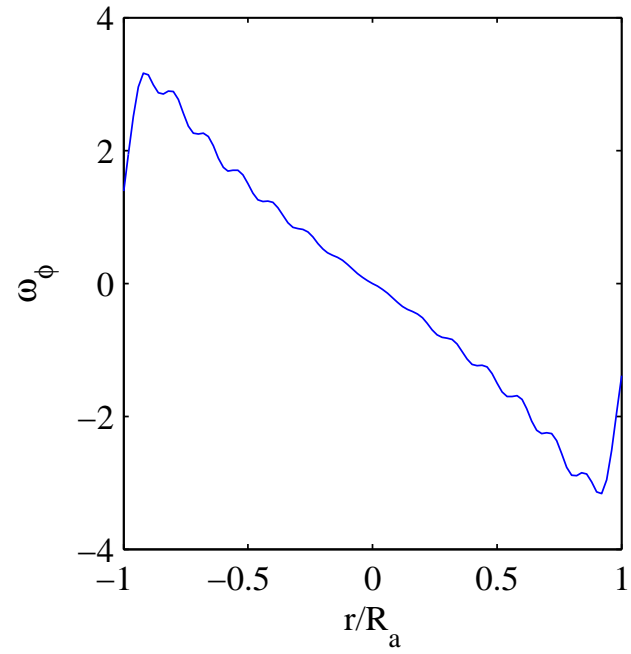

(a) $d t_{o} / 2$

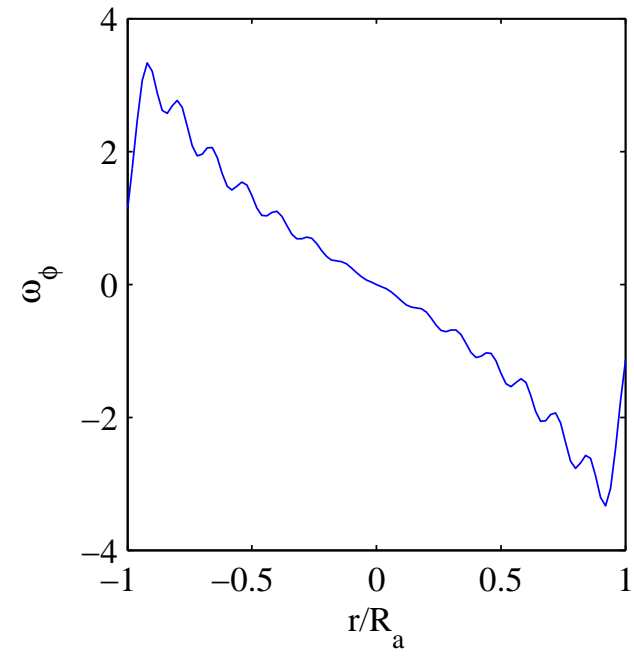

(b) $d t_{o} / 4$

Figure 6.20: Vorticity across the diameter of the actuator disk for (a) halved and (b) quartered time step size.

in a corresponding decrease in the particle core radii to maintain approximately the desired 1.3 overlap between vortex cores. This adjustment of core size results in a reduction in smoothness of the vorticity survey radially across the disk, where the particle spacing wasn't changed at all. The further the time step size is reduced, the louder the noise in the theoretically linear vorticity distribution at the disk becomes. Clearly part (b) of Figure 6.20 has more noise than its counterpart in part (a), or any 
of the matching cases in the previous sections.

In addition to examining the vorticity at the disk, the streamtube vorticity is examined in Figure 6.21. The vorticity field, although not completely smooth, is

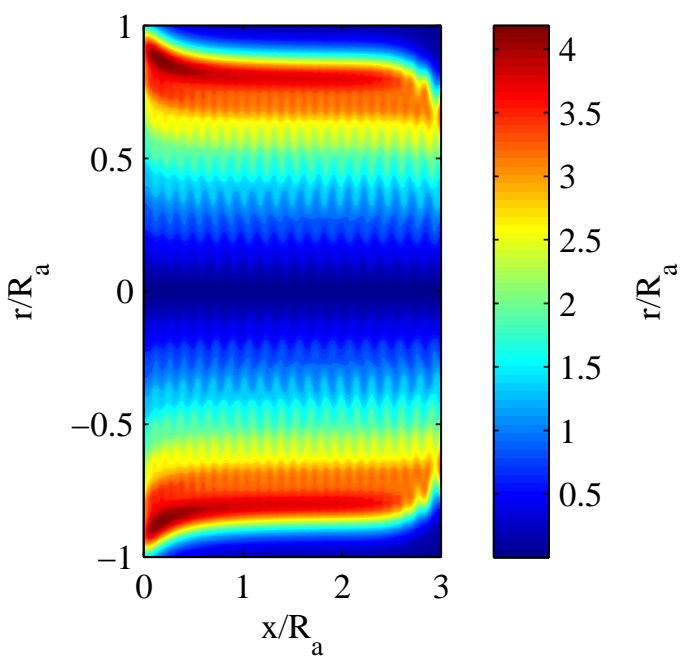

(a) $d t_{o} / 2$

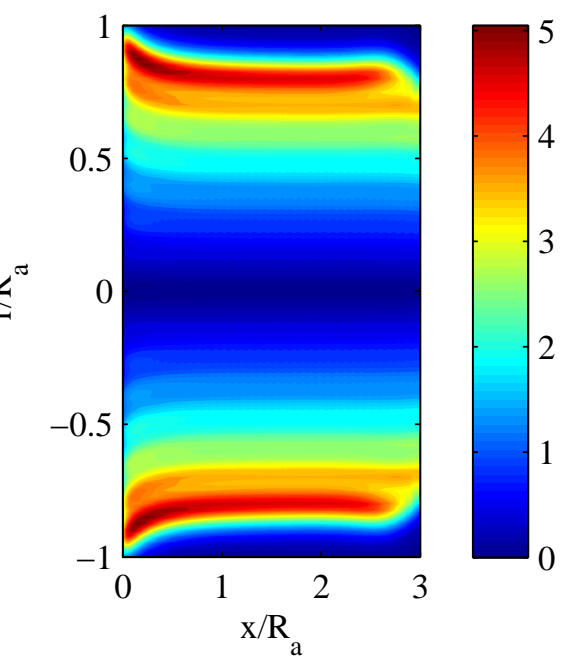

(b) $d t_{o} / 4$

Figure 6.21: Vorticity inside the streamtube downstream of the actuator disk for (a) halved and (b) quartered time step size.

much more so than in any of the previous cases when $n=225$. Much the same way that the decrease in step size caused an increase in noise at the disk, each decreased step size also yields a much smoother vorticity field in the streamtube, as evidenced by (b) of Figure 6.21. The smoothing effect seen is similar to a large increase in particle count which would also reduce core radii and provide a smoother field.

As mentioned, the time step adjustment affects the amount of vorticity in the field, and correspondingly alters the perturbation velocity influences, both radially and axially. In both cases the $\hat{a}$ value is adjusted to most accurately match the axial velocity profile at the disk. This resulted in an $\hat{a}=3.0$ for the halved time step and an 
$\hat{a}=1.65$ for the quartered time step. Figure 6.22 shows the axial velocity comparisons for both time step conditions. Clearly the axial matching at all locations becomes

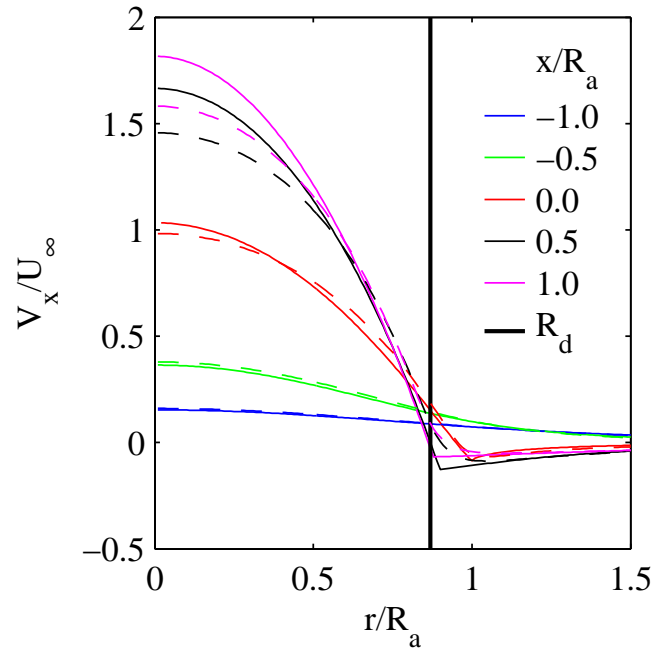

(a) $d t_{o} / 2$

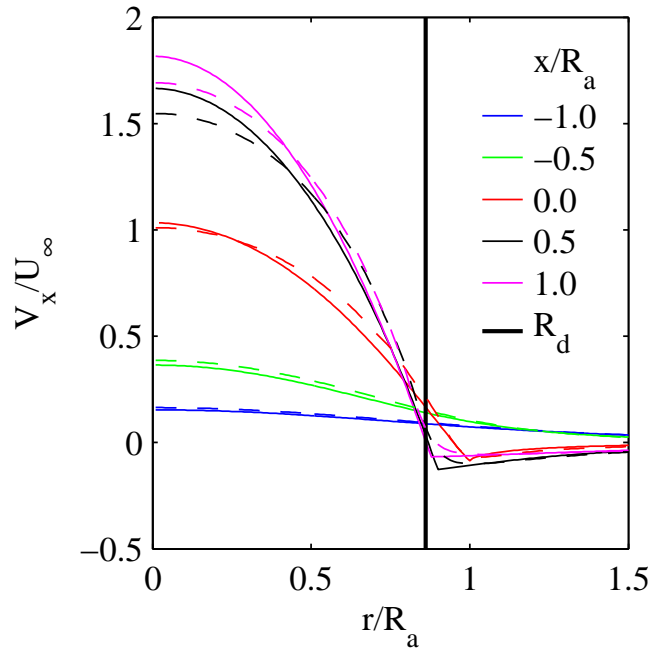

(b) $d t_{o} / 4$

Figure 6.22: Comparison of analytical (solid) and discretized vortex particle (dashed) axial perturbation velocities at several axial positions relative to the actuator disk for (a) halved and (b) quartered time step size.

increasingly good as the time step size is decreased. This makes sense because the reduced time step size is an approximation of the convergence criterion to obtain theoretical value with vortex particles. While a higher number of particles aren't used in the discretization of a single disk, more particles are introduced in the same convected area of streamtube simply because the number of time steps at which particles are released is increased.

Figure 6.23 shows similar results to its axial counterpart. The matching at all axial locations has improved with the half sized time steps, and further improves when time steps are reduced again. In an infinite particle limit it seems reasonable to 
assume that the particle solution would converge to something at least quite similar to that predicted by actuator disk theory, even with the subtle differences between the methods.

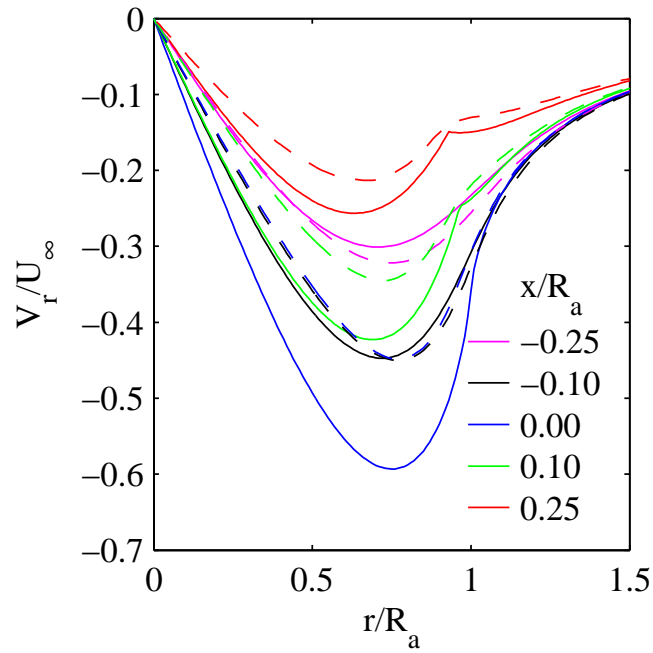

(a) $d t_{o} / 2$

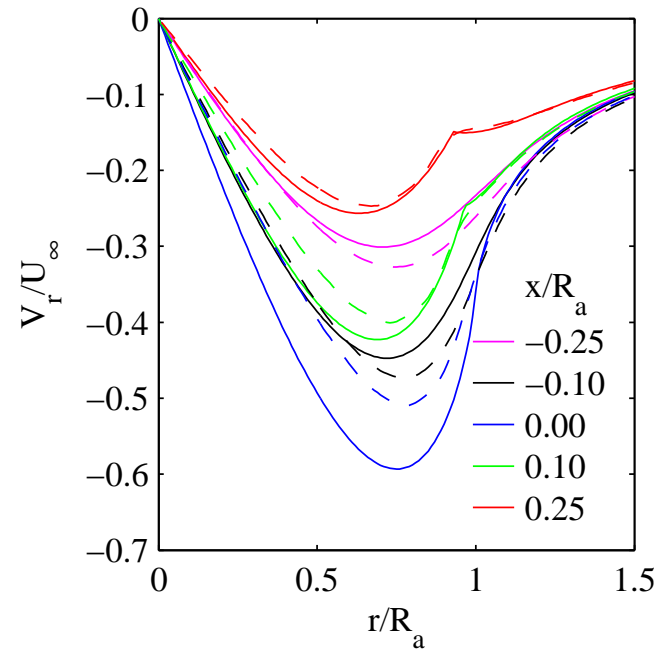

(b) $d t_{o} / 4$

Figure 6.23: Comparison of analytical (solid) and discretized vortex particle (dashed) radial perturbation velocities at several axial positions relative to the actuator disk for (a) halved and (b) quartered time step size. 


\section{Chapter 7}

\section{Aerodynamics Solver: Panel Code}

With a rotational flow model established, and its ability to model actuator disk flowfields verified, an aerodynamics solver that was computationally inexpensive and highly adaptable to different geometries relative to CFD was the only missing piece. This solver was found in the form of the panel code. The benefits of the panel code fit exactly in line with the desired features of the aerodynamics solver needed for the research, while its main shortcoming would be corrected with the introduction of the vortex particle scheme.

\subsection{Fundamentals of Panel Codes}

The panel code has been utilized for aerodynamic analysis since it's creation by

Hess in 1962.61 While at the time it was a computationally expensive method, as computing power has increased with Moore's Law the computational time to run cases has dramatically decreased, making the panel code an ideal tool for modern optimization work. This functionality as a design optimization tool is apparent from 
the work of Choi, Alonso, Walsh, and many others ${ }^{62}$ The advent of CFD methods rendered the panel code obsolete when only accuracy is of concern. CFD methods differ from panel codes though, in that they require a volume grid, which can be quite difficult to construct, especially because cell orthogonality and smooth cell growth rates must be maintained for accurate results. Panel methods on the other hand only require surface grids, which are much easier to construct than volume grids, and have drastically fewer cells. These surface cells are used to solve the linear PrandtlGlauert Equation, a different equation than what is solved in CFD. The use of the Prandtl-Glauert Equation is at the root of why panel codes are less capable, with severe limitations outside the realm of inviscid and irrotational flow, but also why they are less computationally expensive and much easier to set up and run.

\subsection{Theory}

The panel method has its roots in potential flow theory. A series of panels are created that define the surface of the geometry to be studied, and a boundary condition is applied at each of these panels. Each of the panels has a certain element, or set of elements on it, of which there are several types, that exerts some influence on the flowfield. To understand the flowfield around the geometry it is necessary to solve a set of simultaneous equations. Generally a no normal flow boundary condition is enforced at each panel, indicating that there is no flow into the surface of the panel and therefore no flow into the body. To construct the equation for each individual 
panel the influence from all the panels is found at that panel, and the boundary condition is enforced so that there is no net normal flow, with the Kutta condition applied to ensure a unique solution. Once the equation is constructed for each panel then all the equations are solved simultaneously for the required panel strengths to enforce the boundary condition at each panel. The resulting panel strengths are the solution to the problem, and knowing the strength of each panel it is possible to find the resultant potential, or velocity, at any point in the flow field simply by examining the influence from each panel with its corresponding strength.

\subsection{Extension to 3D}

The basic two dimensional panel code becomes more complex when extended to three dimensions. The two main sources of this increased intricacy are the added difficulty of converting to panel coordinates, and the additional dimension of elemental influence that must be accounted for. In addition to these two sources, the extra dimension, while allowing for more complicated geometry, can also make the creation and management of geometry much more difficult.

\subsubsection{Panel Coordinate Transformation}

A MATLAB routine was written to complete the coordinate transformation as needed throughout the overall body of code. The inputs required to run the function are the three panel coordinate vectors, as well as a point of interest to be converted. Two of the panel coordinate vectors are parallel to the surface of the panel and 
simultaneously orthogonal to each other, and the third is normal to the panel. An example of panel coordinates for an arbitrary panel are shown in 7.1 .

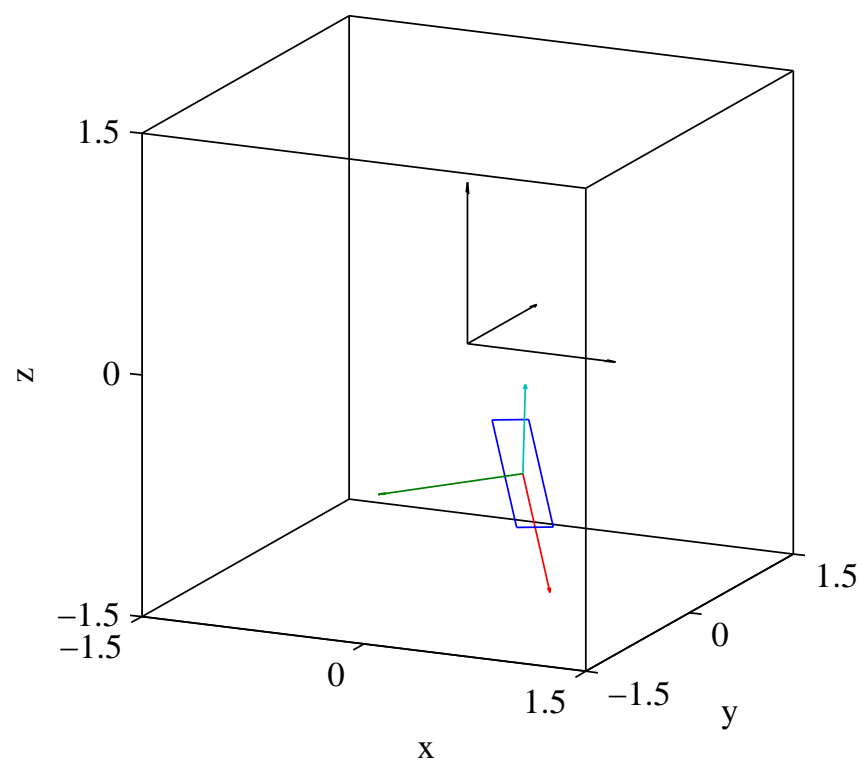

Figure 7.1: Panel coordinates on an arbitrary panel relative to global coordinates.

To convert any point whose global coordinates are known into panel coordinates, it is first necessary to construct a transformation matrix, composed of the three components of each of the three coordinate unit vectors.

$$
\mathbf{T}=\left[\begin{array}{ccc}
l_{1} & l_{2} & l_{3} \\
m_{1} & m_{2} & m_{3} \\
n_{1} & n_{2} & n_{3}
\end{array}\right] .
$$

To obtain the point of interest in the panel coordinate frame the vector point of interest location is multiplied by the transformation vector.

$$
[x, y, z]_{\text {panel }}=[x, y, z]_{\text {global }} \mathbf{T} .
$$

Converting a local velocity vector into global coordinates follows the same procedure 
as the conversion of a vector position. The position vectors of Equation 7.2 are simply replaced by the velocity vectors, $[u, v, w]_{\text {panel }}$ and $[u, v, w]_{\text {global }}$.

The panel coordinate transformation becomes even more complex when converting a velocity gradient calculated in local coordinates back to the global frame. The calculated velocity gradient is the change in local velocity in each of the local coordinate axes. This means the calculated gradient is

$$
\frac{\partial \mathbf{u}^{\prime}}{\partial \mathbf{x}^{\prime}}=\left[\begin{array}{lll}
\frac{\partial u^{\prime}}{\partial x^{\prime}} & \frac{\partial v^{\prime}}{\partial x^{\prime}} & \frac{\partial w^{\prime}}{\partial x^{\prime}} \\
\frac{\partial u^{\prime}}{\partial y^{\prime}} & \frac{\partial v^{\prime}}{\partial y^{\prime}} & \frac{\partial w^{\prime}}{\partial y^{\prime}} \\
\frac{\partial u^{\prime}}{\partial z^{\prime}} & \frac{\partial v^{\prime}}{\partial z^{\prime}} & \frac{\partial w^{\prime}}{\partial z^{\prime}}
\end{array}\right]
$$

where the prime indicates local coordinates, while the desired gradient information is

$$
\frac{\partial \mathbf{u}}{\partial \mathbf{x}}=\left[\begin{array}{lll}
\frac{\partial u}{\partial x} & \frac{\partial v}{\partial x} & \frac{\partial w}{\partial x} \\
\frac{\partial u}{\partial y} & \frac{\partial v}{\partial y} & \frac{\partial w}{\partial y} \\
\frac{\partial u}{\partial z} & \frac{\partial v}{\partial z} & \frac{\partial w}{\partial z}
\end{array}\right] .
$$

A simple conversion like the one in Equation 7.2 won't return the entire gradient to global coordinates. Instead two consecutive conversions are necessary, as shown in Equation 7.5 .

$$
\nabla \mathbf{u}=\left[\nabla^{\prime} \mathbf{u}^{\prime} \mathbf{T}\right]^{T} \mathbf{T}
$$

The first transform of Equation 7.5 is applied as indicated in Equation 7.2 , which returns part of 7.3 to global coordinates, resulting in

$$
\frac{\partial \mathbf{u}^{\prime}}{\partial \mathbf{x}^{\prime}}=\left[\begin{array}{llll}
\frac{\partial u^{\prime}}{\partial x^{\prime}} & \frac{\partial v^{\prime}}{\partial x^{\prime}} & \frac{\partial w^{\prime}}{\partial x^{\prime}} \\
\frac{\partial u^{\prime}}{\partial y^{\prime}} & \frac{\partial v^{\prime}}{\partial y^{\prime}} & \frac{\partial w^{\prime}}{\partial y^{\prime}} \\
\frac{\partial u^{\prime}}{\partial z^{\prime}} & \frac{\partial v^{\prime}}{\partial z^{\prime}} & \frac{\partial w^{\prime}}{\partial z^{\prime}}
\end{array}\right] \mathbf{T}=\left[\begin{array}{lll}
\frac{\partial u^{\prime}}{\partial x^{\prime}} & \frac{\partial v^{\prime}}{\partial x^{\prime}} & \frac{\partial w^{\prime}}{\partial x^{\prime}} \\
\frac{\partial u^{\prime}}{\partial y} & \frac{\partial v^{\prime}}{\partial y} & \frac{\partial w^{\prime}}{\partial y} \\
\frac{\partial u^{\prime}}{\partial z} & \frac{\partial v^{\prime}}{\partial z} & \frac{\partial w^{\prime}}{\partial z}
\end{array}\right] .
$$

The result is a matrix that describes the change in local velocity components with change in global coordinates. Thus, a second transformation is required to return the velocities to global coordinates as well. To do this, the rows of the matrix to 
be transformed must have a consistent panel coordinate term to convert, similar to the fact that the denominator in the first row of Equation 7.3 is $\partial x^{\prime}$ in each term. Transposing the resultant matrix from Equation 7.6 will make the first row consistently contain $\partial u^{\prime}$, the second row $\partial v^{\prime}$, and the third row $\partial w^{\prime}$. Once transposed, the transformation matrix can again be applied, resulting in the final global velocity gradient matrix.

$$
\frac{\partial \mathbf{u}^{\prime}}{\partial \mathbf{x}}=\left[\begin{array}{lll}
\frac{\partial u^{\prime}}{\partial x} & \frac{\partial u^{\prime}}{\partial y} & \frac{\partial u^{\prime}}{\partial z} \\
\frac{\partial v^{\prime}}{\partial x} & \frac{\partial v^{\prime}}{\partial y} & \frac{\partial v^{\prime}}{\partial z} \\
\frac{\partial w^{\prime}}{\partial x} & \frac{\partial w^{\prime}}{\partial y} & \frac{\partial w^{\prime}}{\partial z}
\end{array}\right] \mathbf{T}=\left[\begin{array}{lll}
\frac{\partial u}{\partial x} & \frac{\partial u}{\partial y} & \frac{\partial u}{\partial z} \\
\frac{\partial v}{\partial x} & \frac{\partial v}{\partial y} & \frac{\partial v}{\partial z} \\
\frac{\partial w}{\partial x} & \frac{\partial w}{\partial y} & \frac{\partial w}{\partial z}
\end{array}\right]
$$

\subsection{APAME Panel Code}

An off the shelf panel code called APAME was used to fulfill the needs for a three dimensional code. ${ }^{65}$ APAME stands for Aircraft Panel Method, which is an open source MATLAB code written by Daniel Filkovic, available online. The panel code is based heavily on the three dimensional panel code provided in an appendix by Katz

and Plotkin. ${ }^{66}$ This particular code was chosen because of its open nature and native MATLAB language, which allowed for modifications to easily be made in the same language that the rest of the research was already developed in.

The APAME panel code uses constant strength source and doublet elements throughout a structured mesh. The constant strength influences are reduced to point influences when a user specified distance between panel and point of interest is exceeded. The reduction in order of the influence equations is made for computational efficiency, since the constant strength relationships are more computationally costly. 
The point and constant strength relationships have been demonstrated to converge to the same results as the distance from the influencing element increases, as shown in Appendix $\mathrm{D}$. The resulting matrix equation is

$$
\sum_{j=1}^{N} \mathbf{B}_{j} \sigma_{j}+\sum_{j=1}^{N} \mathbf{C}_{j} \mu_{j}+\Phi_{\infty}=\Phi_{i n}
$$

where $\sigma_{j}$ is the source strength of panel $j$ and $\mu_{j}$ is the doublet strength. The code is capable of handling multiple patches in the geometry. The surface velocities are found with second order finite differences of doublet strength on the interior of the patches, and first order differences along the patch boundaries.

\subsubsection{Boundary Conditions}

APAME uses the same Dirichlet boundary conditions for its combination of sources and doublets as Katz and Plotkin employ in Program 14 in their appendix. ${ }^{66}$ Correct flow physics are obtained by ensuring zero normal flow on the surface, which the Dirichlet boundary condition achieves indirectly. The Dirichlet method is sometimes called the constant potential method because the potential is specified inside the geometry. Although any constant could be used, some allow for simplifications to the system of equations being solved, and the best of these is to set the inner potential

$\Phi_{i n}$ to be equal to freestream potential $\Phi_{\infty}$. This allows a reduction of Equation 7.8 to

$$
\sum_{j=1}^{N} \mathbf{B}_{j} \sigma_{j}+\sum_{j=1}^{N} \mathbf{C}_{j} \mu_{j}=0
$$


where the system of equations is under-determined because there are $N$ equations with $2 N$ unknowns. In order to have a solvable system one set of panel element strengths must be defined. In this case the source strengths are the ones defined before solving the system of equations.

As previously mentioned, to correctly model the geometry in the flow there must be no normal flow on the surface. Rather than explicitly stating this boundary condition in terms of velocity, it can be examined in terms of derivatives of potential as

$$
\frac{\partial \Phi_{*}}{\partial n}=\frac{\partial \Phi}{\partial n}+\frac{\partial \Phi_{\infty}}{\partial n}=0
$$

where $\Phi_{*}$ is the potential just outside the geometry boundary, $\Phi$ is the perturbation potential due to influencing elements, and $\Phi_{\infty}$ is the freestream potential. Equation 7.10 shows that to obtain no normal flow into the body, the normal derivative of the perturbation potential must be equal and opposite to the normal derivative of the freestream potential,

$$
\frac{\partial \Phi}{\partial n}=-\frac{\partial \Phi_{\infty}}{\partial n}
$$

The normal derivative of the freestream potential is the normal freestream velocity, and can be rewritten as $\mathbf{Q}_{\infty} \cdot \mathbf{n}$. Additionally, the discontinuity in normal derivative of velocity potential across a panel is due only to the sources,

$$
-\sigma=\frac{\partial \Phi}{\partial n}+\frac{\partial \Phi_{i}}{\partial n}
$$

and we've set the potential value inside to a constant, so the derivative of $\Phi_{i}$ is zero, 
resulting in

$$
-\sigma=\frac{\partial \Phi}{\partial n} .
$$

Plugging in the definition of $\frac{\partial \Phi}{\partial n}$ results in the definition of the source strengths through

$$
\sigma=\mathbf{Q}_{\infty} \cdot \mathbf{n} .
$$

Now that $\sigma$ is established for all $N$ panels, the system of equations in Equation 7.9 has $N$ equations and $N$ unknowns, and the doublet strengths can be solved for to describe the flow conditions anywhere in the field once the Kutta Condition is accounted for.

The wake method employed by APAME to enforce the Kutta Condition uses panels that are many times the length of the chord to approximate a single semiinfinite wake. Each spanwise group of panels on any geometry that is designated as lifting will have a wake panel attached to it. The length of that panel is set by the user, but is intended to be a great deal larger than the length of the flowfield in question. Each wake panel only has a doublet strength, and that strength is assigned based on enforcing the Kutta condition, where the difference in doublet strength between the upper trailing edge panel and the lower trailing edge panel is set as the wake strength. The doublet wake panels were modeled using a vortex ring formulation to provide the most computationally efficient code possible.

Because the rest of the panel code also avoids complexities such as high order paneling and higher order strength distributions, the approximate semi-infinite wake is an acceptable choice. Additionally, the lack of wake rollup and correspondingly 
complex wake structure drastically reduces the complexity of interactions with the particles shed from the actuator disk.

The wake method does have a major problem associated with it, which was corrected to obtain solutions using the particle scheme. The particle scheme encountered stability issues due to interactions with the wake doublet panels stemming from the singularities that occur on the panel edges. These singularities did not affect results when particles moved around the geometry, likely because the no normal flow boundary conditions prevented particles from getting too close to the singularity. In the wake however, there is not a no normal flow condition. This allows the particles to travel more freely throughout space and encroach on the panel edges. The solution to this problem is to regularize the vortex ring approximating the doublet panels in a similar manner to the way the vortex particle scheme was regularized. Since the doublet surface panels and the doublet wake panels used an identical influencing function, both the surface and wake doublet panels were regularized.

The regularization model used for the panels was based on the model implemented in PMARC ${ }^{67}$ If velocity is calculated inside of a specified distance from a panel edge, the velocity influence from that panel edge was set to zero. The distance from the panel edge was measured in three ways. The first two checks simply measure the distance to the point of interest from each panel edge endpoint. If one of those distances is smaller than the specified core value then the velocity influence from that edge is zero. These first two checks ensure that the point of interest is not within 
a core distance from the two edge endpoints, but do not ensure that the point of interest doesn't lie somewhere between the endpoints but still along the panel edge. This is especially of concern for the wake panels, whose chordwise length is many times the chord of the actual geometry, and therefore much larger than a reasonable core value.

The third condition in PMARC is designed to treat such a situation through the use of a cross product. If the point of interest lies directly on the panel edge, the cross product of the vectors to that point of interest from each panel edge corner point would be zero because the two vectors would be parallel. Thus, as a condition to attempt to prevent singularity issues along the entire panel edge, the cross product of the vectors from each edge endpoint is found, and if that value is within the defined core radius then the velocity influence is again set to zero.

While this check is necessary to ensure that no singularity issues occur, it is difficult to implement when some panel edges are much longer than others, as is the case for wake panels. This is because the magnitude of one of the vectors from the panel edge corner points becomes extremely large simply due to the length of the panel. The result is that even for extremely small distances away from the panel edge, the cross product term is very large, due to the nature of the long edge. This cross product value is larger than any reasonable core input size, and so singularities often occur. This issue requires special attention when setting wake panel length, so as to ensure that a reasonable core size will prevent singularity issues from arising. 


\subsubsection{Geometry Validation}

One of the additions that was made to APAME for the benefit of this research was the creation of a visual check that the geometry was imported correctly. The ability of APAME to handle geometries with multiple patches makes it very versatile, but it also necessitates a tool to check the validity of the imported geometries. Panel coordinate vector generation depends on the orientation of the matrix containing input coordinate data for the various patches of th geometry. As such, if the input coordinate matrix is not constructed and oriented correctly, the panel normal vector may be calculated with the wrong sign, which leads to incorrect panel code results.

To correct this shortcoming a specific plot was created that displayed all the paneled patches of the geometry, along with the local panel coordinate vectors at the collocation point of each panel. This plot is generated for any input geometry, and the user can then visually check to make sure that the normal vectors for each panel are extending out of the surface of the geometry from the collocation point. This allows the user to feel comfortable that all coordinate transformations taking place throughout the body of the panel code are transforming to the correct coordinate system, and with the correct sign orientation. Samples of this plot are seen in Figure 7.2, where (b) shows incorrect normal vectors, with the normal vectors directed inside the geometry on the root of the wing in the top left corner, while those on the wing in the bottom right corner correctly project outward. The incorrect normal vectors obviously result in incorrect panel influences, and so the ability to visually 


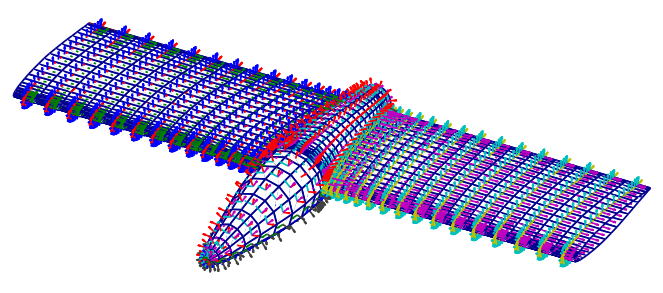

(a) Correct Panel Normals

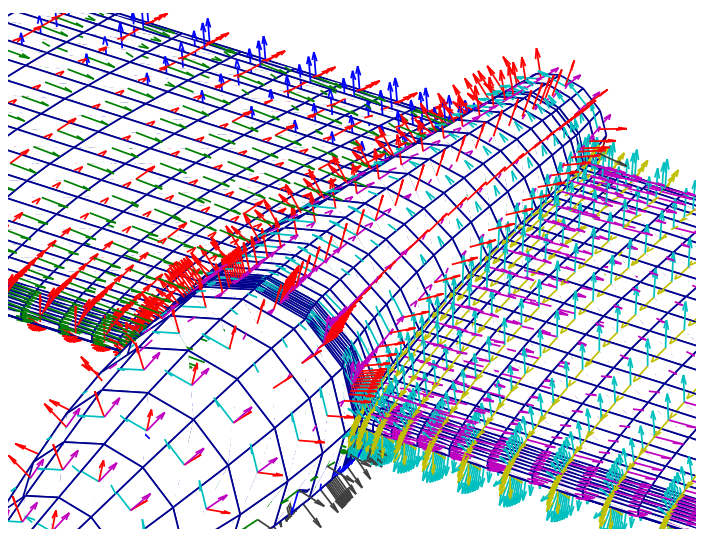

(b) Incorrect Panel Normals

Figure 7.2: An example of a distribution with inward facing normal vectors (dark blue) on the wing in the upper left and correct outward normal vectors (teal) on the wing in the bottom right.

observe correct vector orientation is quite useful.

\subsubsection{Field Velocity Survey}

In many cases it is essential to know more about a particular flow solution than just the velocity and pressure coefficients on the surface of the geometry. After all, part of the power of a panel code is that the solution defines the entire flowfield, not just that of the surface. To be able to obtain field data based on the solution of panel source and doublet strengths was deemed important enough to edit the APAME code once more, this time by creating a set of functions to calculate off-body velocity in MATLAB.

To understand the velocity anywhere in the flowfield it is only necessary to know the strengths assigned to all the panels in the geometry. The influence from each of these panels is then summed at any domain point to understand the flow conditions there. The influence from any individual panel depends on the element type and the 
distribution type associated with that panel. In the case of APAME that is constant strength source and doublet panels, which become point source and doublet panels when a user specified farfield condition is met. Equations that describe the influence from both constant strength and point sources and doublets can be found in the VSAERO theory document. $\underline{68}$

\subsubsection{Streamlines}

Streamlines are an important visualization tool to qualitatively check the reasonableness of the panel code solution, and also to determine if the field velocity survey was correctly implemented. Two separate streamline methods were built into the code, with one using existing MATLAB functions, and the other using a time stepping approach.

The first streamline method added into the code was based on the existing MATLAB function called streamline. This particular function requires a Cartesian grid to be created as an input, which can pose some difficulties when trying to generate it while not placing any nodes inside the geometry. There isn't a particularly quick solution to the Cartesian grid problem, so streamlines that enter the body must just be ignored as being rooted in the fact that the MATLAB function is using fictitious velocity values inside the body in the construction of its streamlines. Figure 7.3 shows streamlines generated using the built in MATLAB function. Both the streamlines and the grid points that data was taken at to construct streamlines are shown. It is clear that grid points exist inside the airfoil surface as well as outside, and thus the 
influence at grid points inside the airfoil cause streamlines near the body to cross into it, which clearly is not physical.

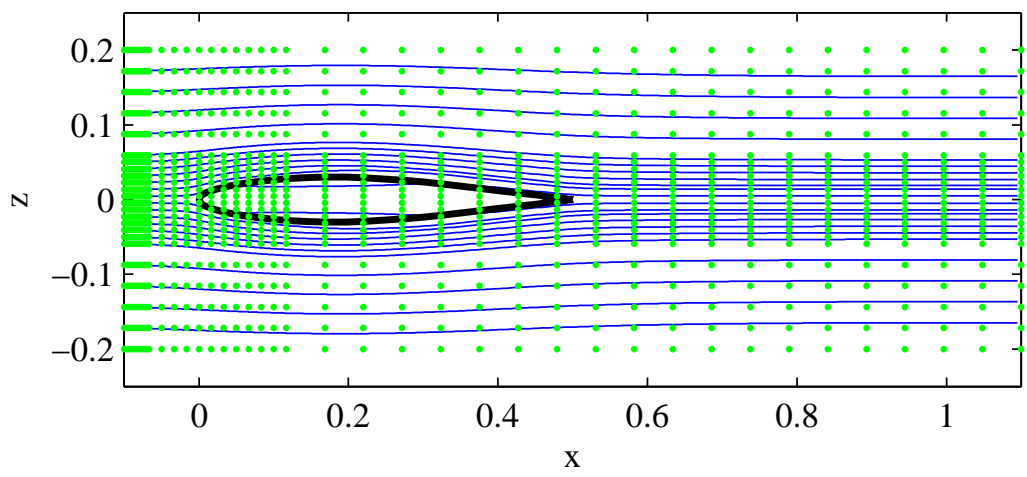

Figure 7.3: An example of streamlines generated using the MATLAB function.

To avoid the problems associated with the Cartesian grid requirement for the MATLAB streamline function, another method of streamline plotting was also implemented. This method uses an assumption that the flowfield is steady to equate the definition of a pathline with the definition of a streamline. For this assumption to be valid the streamlines cannot be generated within the timestepping of the particle method. This is acceptable because the streamlines are only of interest after the simulation has been run. An additional caveat to the assumption is that the unsteady element of the vortex particle-panel code, specifically the start up wake, must be convected far enough downstream to avoid being a major influence on the streamlines in the area of interest. To restate this in another way, streamlines should only be generated in areas where the flow has reached a pseudo-steady state value, which requires unsteady influences from the wake to be negligible.

The grid-free streamline method requires a set of specified streamline start lo- 
cations, as well as a specified timestep size. The method then places a massless, non-influencing particle at each streamline start location that is simply convected through the fluid. To accomplish this, the velocity influence is found at each element start location, and then the elements are convected using the summed influence of all panels and a selected time stepping scheme, such as a Runge-Kutta solver. The implementation currently applies basic Euler time stepping, but the third order low storage Runge-Kutta Scheme of Section 4.4 could be implemented in its place. This method allows for infinite resolution of streamlines through increasingly small time steps, as well as providing a way of avoiding any streamlines entering into the geometry as long as time step size is chosen correctly. The method does carry a cumulative error with it though. That is to say that any error incurred at the first time step results in an element being in an incorrect location at the start of the next time step, and as such the element will be even further from its theoretical path if additional error is accrued in the second time step. For the purposes of this research where various geometries would be used, and grid creation without placing points inside the geometry is difficult, this second method is by far the most useful, and a sample of it can be seen in Figure 7.4 .

\subsubsection{Surface Pressure Coefficient Interpolation}

The AGARD wind tunnel experiment had pressure measurements at specific ports

all along the nacelle and wing. In order to accurately compare the panel code results with those of the wind tunnel test, an interpolation routine was required. This was 


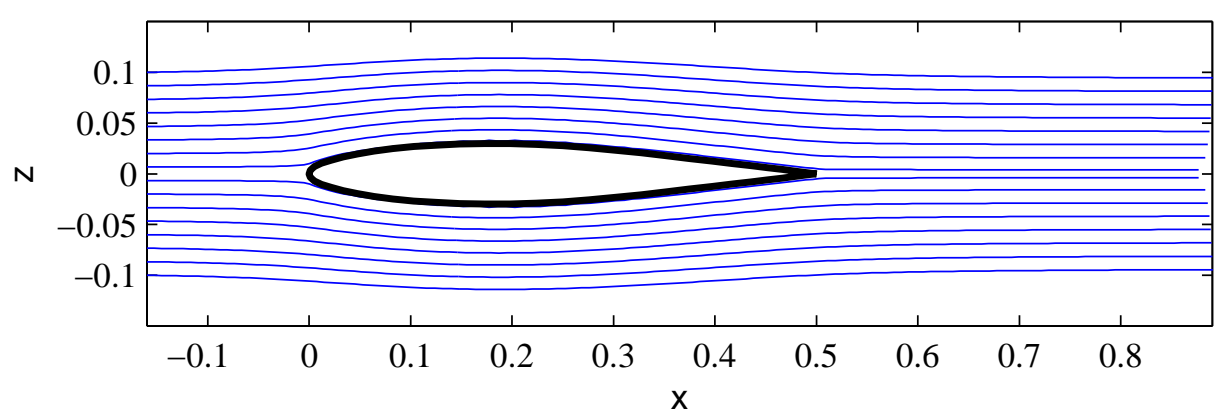

Figure 7.4: A 2D example of streamlines generated from the time stepping technique around a 3D symmetric wing panel solution.

necessary because pressure coefficient data would only be known on the surface of the paneled geometry at each panel collocation point. There was no guarantee that the collocation points would fall at each of the experimental pressure port locations.

\section{Wing Interpolation}

An interpolation method was applied to the wing, where data was interpolated in terms of $i$ and $j$ matrix indices instead of $x, y$, and $z$. The structured nature of the paneling made interpolation in the surface feasible, and likely the best possible solution in terms of elegance and accuracy. Interpolation in the $i-j$ frame starts with finding the $[i, j]$ matrix indices that identify the collocation points bounding the point where pressure coefficient data is desired. Once the bounding indices are known, linear interpolation is carried out independently in the $i$ and then $j$ dimensions.

\section{Nacelle Interpolation}

Since the wind tunnel pressure taps fall in axial rings along the nacelle, with multiple ports appearing at each single axial slice, the collocation point data was 
interpolated to create a ring of data at the axial slice location of interest. This means that each collocation point was interpolated using linear interpolation to create an interpolated ring of pressure coefficient data at the desired axial location. Next, rather than interpolating in $x$ and $z$, the interpolated collocation data is interpolated using $\theta$.

\subsection{Summary of Contributions to APAME}

As demonstrated, a variety of contributions have been made over the course of this research to the APAME panel code package. The specific contributions span from various added plot features to additional calculations. The original surface contour plots generated in APAME used edge centered data, which resulted in collocation data being plotted on panel edges. These plots were changed to cell centered plots so that the collocation data was actually plotted at the collocation points. Additionally, the geometry validation plots were created to help the user visualize whether panels had been imported correctly, which was a feature previously unavailable.

Beyond the newly added plots, several additional calculations were also added into the APAME solution. The streamline capabilities using both the MATLAB function and the timestepping scheme were completely new additions, as well as the plots that accompany them. Off-body velocity influence equations were created for point and constant strength source and doublet elements. Additionally, the panel skewness correction originally implemented in APAME was simply an approximation. A matrix 
method to exactly solve for velocity components on a skew panel was implemented instead to provide the best possible answers. Lastly, a pair of interpolation routines, one for radial interpolation on axisymmetric bodies like the nacelle, and one for inmatrix interpolation used on the wings, were incorporated with APAME to use as an accurate tool to compare calculations with experimental data at any point on the surface. 


\section{Chapter 8}

\section{Panel Code-Particle Scheme Integration}

One of the most fundamental components of this research lies in the correct combination of the panel code with the vortex particle scheme. Each independent code both affects and is affected by the other, and to correctly capture the desired interference effects between the two models they must be able to easily communicate. The influence of each scheme on the other can be examined independently, with the time evolution of the solution providing feedback to both schemes in a highly coupled manner. Particles passing quickly over panels will contribute to the dynamic pressure experienced on that paneled surface, while simultaneously particles will receive adjusted strengths through stretching due to their proximity to the nearby panels, as well as repulsion due to the panel velocity influence. 


\subsection{Panel Influence on Particles}

The more straightforward of the interactions is that of the panels on the particles. The traditional particle scheme is a basic $N$-body problem, where for each particle the influence from all the other particles is found. The integration works because each particle is an influencing element. In APAME each panel also exerts two influences, one from the source strength and one from the doublet strength. As such, in addition to looking at the velocity and stretching influence from each particle, the velocity and stretching influence from each element type on each panel is also found.

Because APAME and VSAERO share the same panel types it is possible to use the velocity influence equations laid out in the VSAERO theory document to calculate velocity influences from panels in APAME. The relationships provided in the VSAERO theory document detail the exact procedure to calculate velocity influences from both point and constant strength source panels and doublet panels, so no rep-

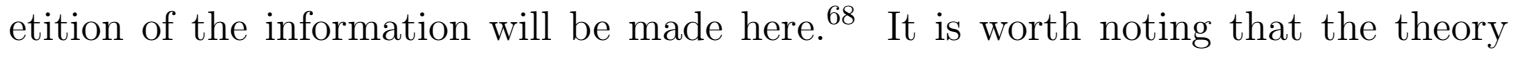
document uses the fact that a constant strength doublet panel is equivalent to a panel whose bounding edges are vortex filaments. In this case, a singularity exists whenever the point of interest is in line with a panel edge. The theory document then uses an alternative expression to reduce the occurrence of the singularity to only when the point of interest lines directly on a panel edge. This makes the singularity issue much less of a concern, as the vortex particles are discretized to avoid placement inside any geometry. 
In addition to influencing the velocity of each particle, the panels also influence the velocity gradients at each particle location. Because the velocity gradient isn't a critical piece of information for most other applications, there aren't well documented derivations of the analytical derivatives of these velocity equations. The nature of this research requires particles to travel extremely close the geometry through the streamtube-geometry interaction, and therefore it is critical to understand and account for the stretching influence stemming from the panels. Complete derivations of the stretching due to point and constant strength source and doublet panels can be found in Appendix C.

\subsubsection{Particle Strength Update Equation Verification}

Again because of the undocumented nature of the analytical derivatives of velocity it was important to ensure that the relationships were correctly calculated. To do this the analyical velocity gradient equations were coded in MATLAB, along with numerical finite difference derivatives of the original velocity and potential influence equations. Then a single simple panel was created, first using a flat panel only in the $\mathrm{x}-\mathrm{y}$ plane, then a panel at an arbitrary orientation, making sure that it had $\mathrm{x}$, $y$, and $\mathrm{z}$ differences between the panel corner points. The influence from the single panel was found at eight different points in space, one corresponding to each octant to make sure that all of the signs in the equations were correct.

This comparison examined the accuracy of 36 different differentiated velocity terms. This is because there are four contributors to velocity influence in the re- 
search; point source and doublet elements and constant strength source and doublet elements. For each of these four contributors there are nine derivatives necessary to fully understand the stretching because of the gradients of the $\mathrm{x}, \mathrm{y}$, and $\mathrm{z}$ components velocity. Each of these nine derivatives was checked for each of the four element types at each of the eight survey points, and the difference between the analytical and numerical values were approximately equal in each case. Figure 8.1 shows an example of an arbitrarily oriented constant strength source panel in space, with nine survey points around it, including one in each octant, and an additional point in the plane of the panel, as well as the velocity vectors corresponding to each point. All of the stretching equations were verified by comparison with finite difference derivatives of velocity and potential, as discussed in Appendix D.

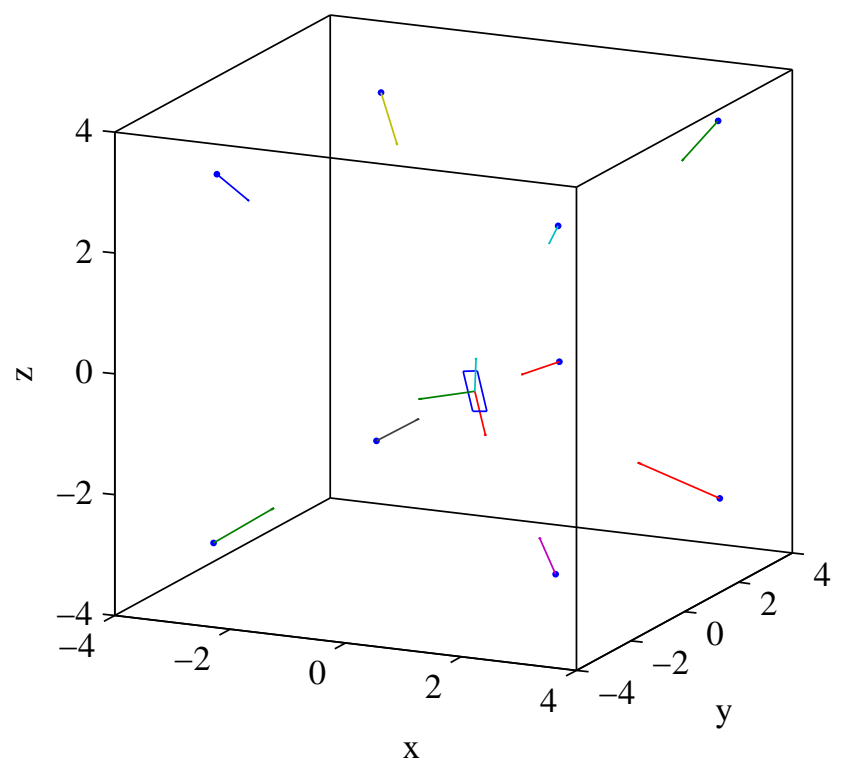

Figure 8.1: Example of 9 survey points relative to the single constant strength source panel, as well as the velocity vectors corresponding to each point.9] 


\subsection{Particle Influence on Panels}

The particle influences on the panels are slightly more subtle than the panel influences on the particles. Each panel strength is assigned based on meeting the boundary conditions of the panel code. One of the two boundary conditions is that there should be no normal flow into panels, which is approximated by making sure that there is no normal flow into each panel at each collocation point. Traditionally in a combined source and doublet panel code, in conjunction with the inner potential being set to a constant, this boundary condition takes the form of

$$
\sigma_{j}=-\mathbf{n}_{j} \cdot \mathbf{Q}_{\infty}
$$

With the addition of vortex particles, eliminating normal flow to the panel from freestream velocity is no longer sufficient. When the particles are included their velocity influence must be accounted for in the same manner as the freestream velocity. This results in the new boundary condition stating

$$
\sigma_{j}=-\mathbf{n}_{j} \cdot\left(\mathbf{Q}_{\infty}+\sum_{q} V_{\text {particle }}^{q}\right)
$$

where now in addition to the freestream velocity, the velocity influence from all $q$ particles is summed at the point, and that resulting total velocity must have no normal flow to the panel. A nearly identical boundary condition has already been proven by Conway! 15

The second boundary condition, which sets the inner perturbation potential to zero remains unchanged with the addition of the vortex particles. The resulting 
summation problem remains

$$
\sum_{j=1}^{N} \mathbf{B}_{j} \sigma_{j}+\sum_{j=1}^{N} \mathbf{C}_{j} \mu_{j}=0
$$

With the source strengths specified by Equation 8.2, Equation 8.3 is now solved for the doublet strengths.

\subsection{Steps To Get A Solution}

The solution for any geometry with any number of actuator disks is the same, and can be summarized in tabular form. The steps are quite similar to those used by Willis in FASTAERO ${ }^{1}$ in Table 2.1. The specific steps for the current method are supplied in Table 8.1 .

Table 8.1: Steps to generate a particle-panel code solution for propeller-airframe interaction.

\begin{tabular}{|c|c|c|}
\hline Step & Description & Sections \\
\hline 1 & Solve for panel strengths and wake potential jump & 7.47 .4 .1 \\
\hline 2 & Release actuator disk particles for current time step & 4.110 .1 \\
\hline 3 & Determine velocity and stretching for particles from panels & $\mathrm{C} / \mathrm{D}$ \\
\hline 4 & Determine velocity and stretching for particles from particles & 3.5 \\
\hline $4 \mathrm{a}$ & If necessary, compute pressures and forces on the body & $\mathrm{N} / \mathrm{A}$ \\
\hline 5 & Update particle positions and strengths in the streamtube & 4.4 \\
\hline 6 & Determine particle velocity influence on body & 8.2 \\
\hline 7 & Return to Step 1 unless iteration criterion is met & 6.2 \\
\hline
\end{tabular}




\section{Chapter 9}

\section{AGARD Wind Tunnel Test}

The culminating goal of this research was to validate the method against experimental data. AGARD CP306 provided just such a study to validate against, with the presentation of a series of well documented wind tunnel tests specifically run to be a benchmark for CFD cases ${ }^{9 \sqrt[22]{23}}$ Details about the experiment, along with all the data recorded during the tests, was obtained and used for comparison. To compare computational results of the research with the experimental method it was first necessary to understand the details of the tests and how they were conducted. The coordinate system applied in this chapter and the following chapter is chosen to maintain consistency with the AGARD experiment coordinate system. In this coordinate system, $\mathrm{x}$ is the axial, $\mathrm{y}$ the spanwise coordinate, and $\mathrm{z}$ the vertical.

\subsection{Wind Tunnel Test Information}

The tests were conducted on four different configurations, each of which contained a nacelle and propeller. The first configuration was an axisymmetric nacelle with a 
propeller mounted on it. The second configuration had the same nacelle as the first, but the nacelle was now mounted in the center of a wing with a 2.06 meter span wing using a symmetric NACA $63_{(10)} A-012$ airfoil section, which is a scaled up version of the NACA $63 A-010$. The third configuration was a nonaxisymmetric nacelle with a propeller, and the fourth was the new nacelle mounted on the same wing as in the second configuration. Only the first and second configurations were examined in this work and only at zero degrees angle of attack, but the third and fourth configurations could also be examined with the method, both at zero and nonzero angles of attack.

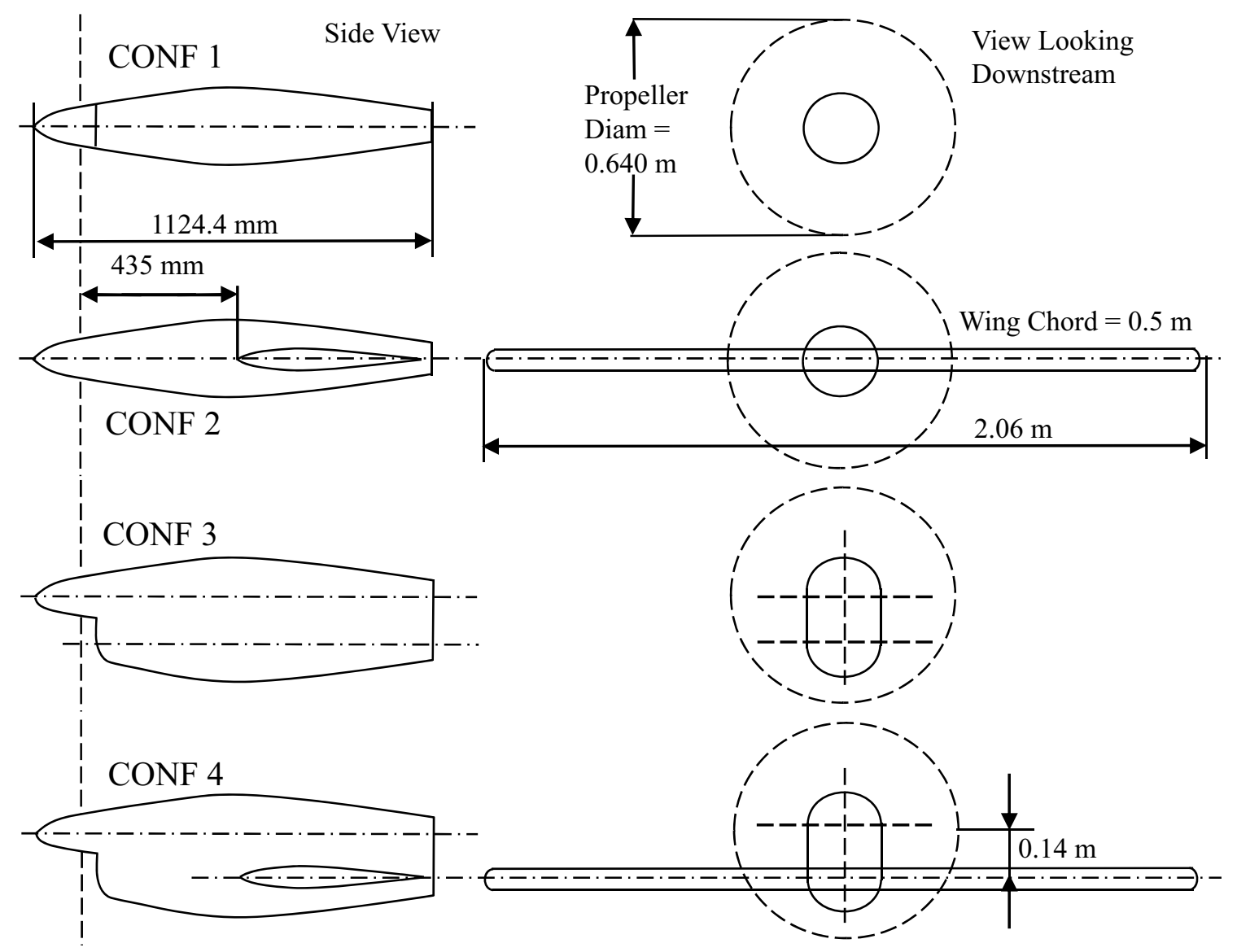

Figure 9.1: The four unique configurations from AGARD wind tunnel tests 9 
All of the configurations used an identical propeller. The propeller had four blades, and was a 20 percent scale model of the original propeller mounted on the SAAB 340 commuter aircraft. The model propeller diameter was 0.64 meters, and the entire propeller model was built of aluminum. Samuelsson felt that the particular propeller exemplified a highly loaded propeller typical for the newest generation of medium speed commuter aircraft when the test was conducted in the late 1980's. 22

The measurements on the model were taken through a variety of means. The model itself was tail sting mounted, and the sting controlled the angle of attack and side slip angle through servo motors. The thrust and torque measurements were made through strain gauge elements mounted on the propeller shaft inside the spinner. The RPM was measured through a magnetic pickup mounted on the motor. The nacelle and wing surface pressures were all measured using the Scanivalve technique. Additionally, a five hole probe was used to take field velocity data. The five hole probe was calibrated in the same wind tunnel used for the experiment. It was run at a variety of different speeds and angles of attack, and all data gathered with it was sampled multiple times and then averaged to reduce noise. The effects of the probe on overall data was measured by taking both surface pressure and probe measurements at the same time, and the effect was deemed to be negligible.

The wind tunnel used was the FFA Low Speed Wind Tunnel, run by The Aeronautical Research Institute of Sweden. The tunnel is a low speed, closed circuit tunnel with an electrical motor driven fan capable of speeds of up to $85 \mathrm{~m} / \mathrm{s}$. The test section 
diameter is $3.6 m$, while the length is $8 m$, with a typical boundary layer thickness of between $0.01 \mathrm{~m}$ and $0.02 \mathrm{~m}$. Configuration 3 can be seen relative to the wind tunnel in Figure 9.2 .

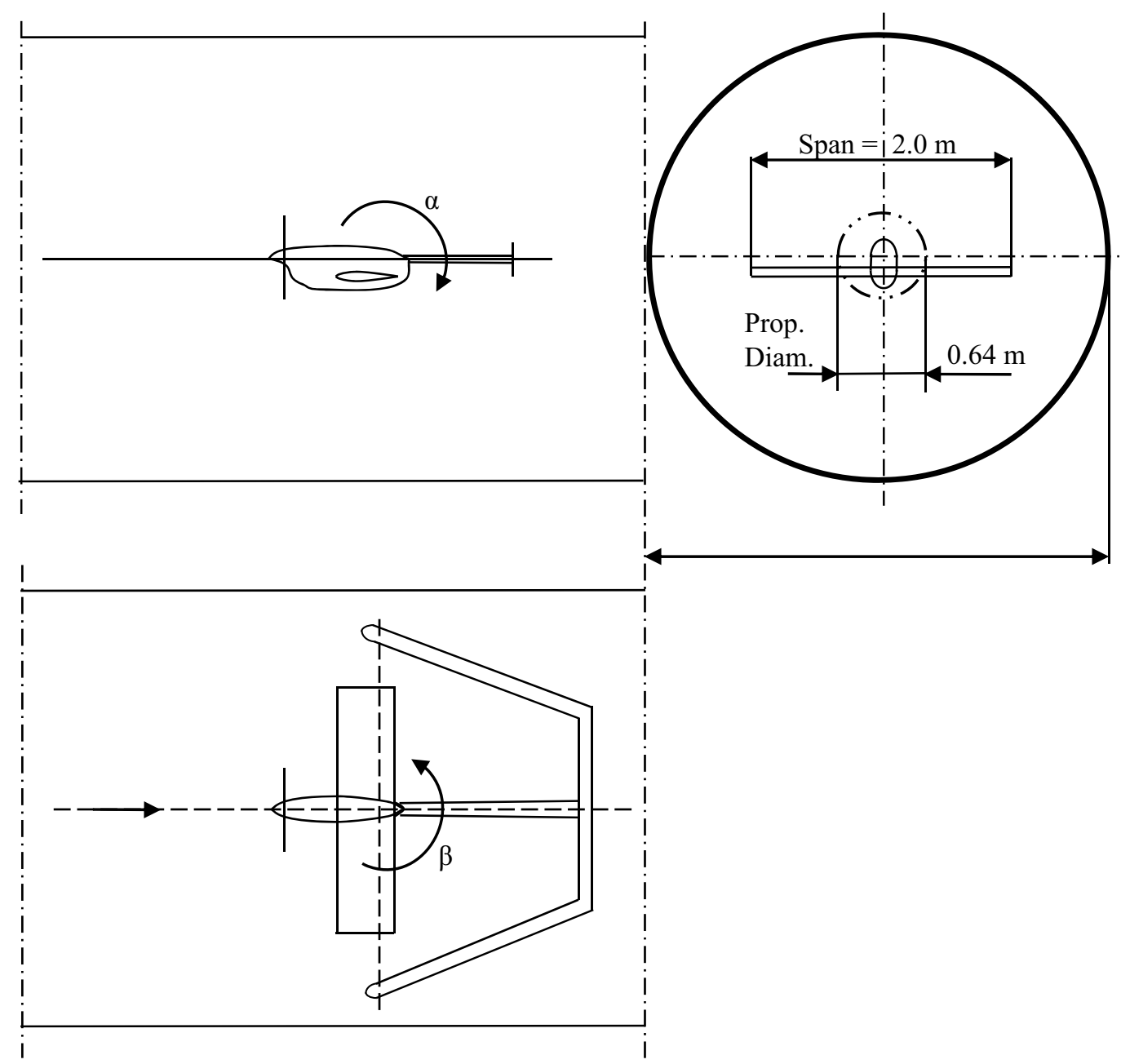

Figure 9.2: Configuration 3 model size relative to the wind tunnel ${ }^{9}$

Of important note is the fact that all the data has been corrected for wall effects to simulate "free-air" conditions. Corrections were made using the Prandtl-Glauert compressibility correction as well as Glauerts' wind tunnel/propeller interference correction, with corrections of $-2 \%$ applied to the freestream and flowfield velocities, 
as well as the advance ratio. These reductions account for the increases in velocity that an area constricted flow like a wind tunnel experience in conjunction with model blockage. No corrections were implemented to account for interference from the sting and strut equipment, nor for the measuring equipment such as the five hole probe.

The tests relevant to the work of this research were conducted at zero angle of attack and side slip, with a freestream velocity of 50 meters per second. The propeller blade pitch at 75 percent blade span was 29 degrees, and the propeller was turned at 6650 RPM, with a thrust coefficient of 0.23 , and advance ratio of 0.70 , and a power coefficient of 0.25. Samuelsson chose this test case because the power conditions are reflective of those used during climb-out, except in this case the angle of attack and flap deflection angles are both zero.

\subsubsection{AGARD Experiment Shortcomings and Differences}

Although the AGARD experiment conducted on propeller-airframe interaction is quite a valuable tool as a benchmark for numerical simulations, it does have inherent shortcomings. The shortcomings stem from various different parts of the experimental procedures and results, but all of them have the potential to contribute to differences between the experimental data and numerical simulations attempting to match it. In addition to the shortcomings of the experiment, there are also some distinct differences between the flow physics of the experiment and what is modeled in the numerical simulations presented here. 


\section{Shortcomings}

One weakness of the AGARD experimental data is the accuracy of the pressure port measurements. When examining Configuration 1 with the propeller off there is some variation around the surface of the nacelle, when in fact the flow should be completely axisymmetric. Likewise, when examining Configuration 2 there was again variation in pressure coefficient values not related to the true flowfield. In addition, the pressure coefficient measurements on the wing surface were not symmetric between the upper and lower surface. This asymmetry must be an error because the wing used a symmetric airfoil at zero degrees angle of attack. The differences here could stem from any number of sources, including surface imperfections on the geometry or in the pressure taps, or asymmetric flow inside the wind tunnel.

There is also some concern about the way the measurements were taken. Since the experimental flowfield was unsteady due to the continual rotation of the propeller, all data measurements were time averaged. It isn't clear how the experimenter took data to ensure that data capture occurred over intervals of whole periods of propeller rotation. If only a portion of a period was captured in the time averaging, the results wouldn't reflect a time average of the complete flow, but only of a portion of one period while neglecting the occurrences during the remainder of the rotation period. The error incurred from the difficulty in capturing data in exact periods of propeller rotation is demonstrated in a report on the experiment, 22 which presents figures showing the variation in data during repeatability studies to be quite significant, 
especially when considering change in stagnation pressure in the streamtube.

An additional source of error is related to the applied corrections. No validation of the corrections that were applied was shown in the reports, although the presence of corrections indicates that the experimenter felt that there would in fact be a need for them. Because no validation is shown it is difficult to assess how well the corrections do, and therefore it is possible that some difference still exists between the corrected data from the wind tunnel and the data that would be captured in a wall-free case.

Lastly, there is concern over the use of the five hole probe for measurements inside the slipstream. Takahash ${ }^{69}$ noted that five hole probes accurately measure flow angularity only in low turbulence flowfields. The streamtube close behind a propeller rotating at high speeds may well be outside the limits of what a five hole probe can accurately measure. Between the periodic presence of shed wake from the propeller blades and the unsteadiness of the flow in general, it is likely that the wind tunnel data itself does not exactly reflect the true flow physics.

\section{Differences}

A major difference between the experimentally captured data and that produced by the method presented here lies in viscous effects. Viscous effects play a large role in complex flowfields such as the case of propeller-airframe interaction. The numerical method discussed here is intended to be a conceptual design and optimization tool, and so although it is currently possible to numerically model some viscous effects, the costs associated with that capability were deemed too high, and no viscous model was 
included in the aerodynamic solver. The particle scheme has the capability to model viscous diffusion within the flowfield it describes, but that requires the use of the particle strength update, which caused instabilities in several of the cases examined for reasons that will be discussed more in Chapter 10. The lack of viscous models meant that there could be no good resolution of vorticity inside the boundary layer, nor could the numerical simulation capture any vorticity lost to viscous dissipation.

Another large difference between the two methods is the way in which stagnation pressure jump is treated. The surface pressure ports in the experiment inherently capture the exact stagnation pressure jump because they measure the static pressure, $p=p_{o}+q$. In the numerical simulation, the initial pressure measurements come only from the dynamic pressure term, so an additional factor must be added to account for change in stagnation pressure, as discussed in Section 5.3. This requirement for explicit treatment of the stagnation pressure change in the numerical scheme has the potential to introduce error into the static pressure values.

As mentioned in the discussion of the experimental shortcomings, there is some concern over the exact time averaging procedure employed in the experiment. Because the actuator disk used in the numerical method is by nature time averaged, any discrepancies that exist in the experimental time averaging would contribute to differences in results, simply because of whether the flowfield is pseudo-steady or unsteady. 


\subsection{Configuration 1 Panel Code Calibration}

To truly understand the accuracy with which the vortex particle actuator disk model captures pressure coefficient changes over the nacelle, it is necessary to understand the inherent error existing in the model prior to inclusion of the vortex particles. To accomplish this, the paneled geometry was run through the APAME panel code without any particle actuator disk present, and compared with AGARD results with the propeller off. The paneled geometry of Configuration 1 is shown in Figure 9.3 . The nacelle has 40 rings of panels down the spine of the nacelle with 16 panels in each ring. This can be thought of as a $40 \times 16$ axial-radial distribution of panels. The panels are intentionally clustered at the same axial locations as they would be in the second configuration to cluster the leading and trailing edges of the wing. The panel code does an good job of predicting the surface pressures over nearly the entire nacelle for this nonlifting geometry.

\subsubsection{Surface Pressure Coefficient Measurements}

Figure 9.4 and Figure 9.5 show a comparison of the experimental and panel code results. The experimental results are shown in vector format, where the vector starts at the pressure port location and extends a distance reflective of the pressure coefficient times a scaling factor, which is in this case 100. Vectors pointing into the nacelle indicate positive pressure coefficients, while vectors pointing away from the nacelle indicate negative pressure coefficients. The data from the panel code is found 


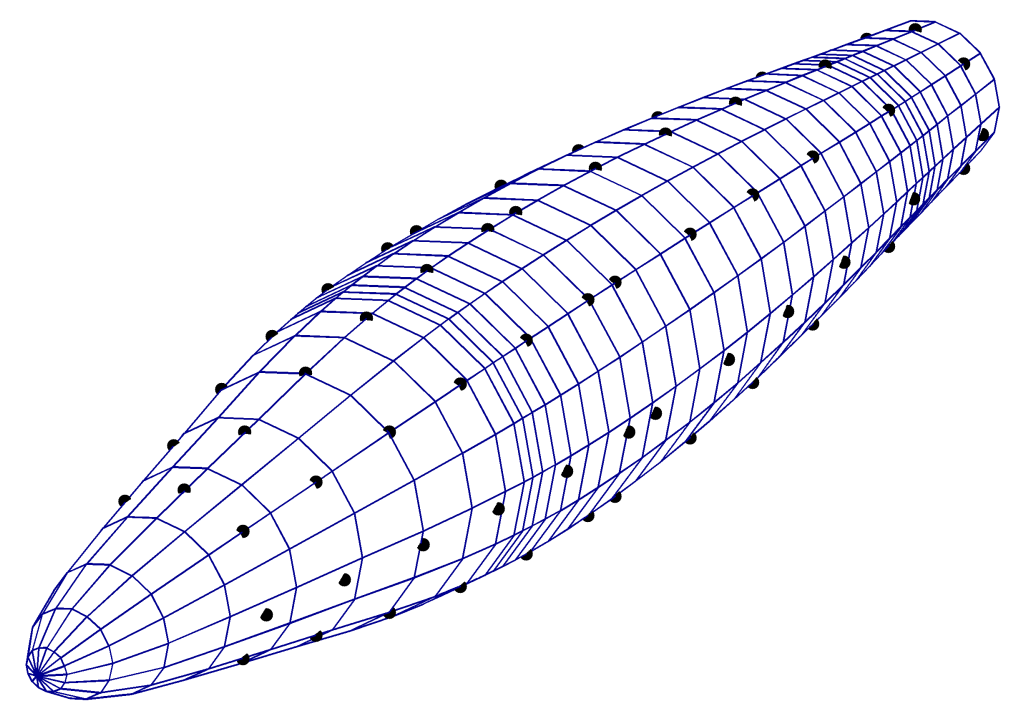

Figure 9.3: Paneled model of AGARD Configuration 1 with pressure port locations shown as black dots.

by interpolating between calculated values located at collocation points. The figures are intended to demonstrate that the pressure coefficients calculated from the panels agree well with the wind tunnel data with the propeller off, which is demonstrated by the extreme similarity between the vector pressure tap data and the interpolation lines. The data does not match as exactly at $x=925 \mathrm{~mm}$, and this is likely due to the fact that the trailing edge was not paneled at all, since the panel code would poorly model the flow around the discontinuous edge at the end of the nacelle.

\subsection{Configuration 2 Panel Code Calibration}

The same procedure used on Configuration 1 can be replicated for the second configuration, whose paneling is seen in Figure 9.6. The panel density is slightly 


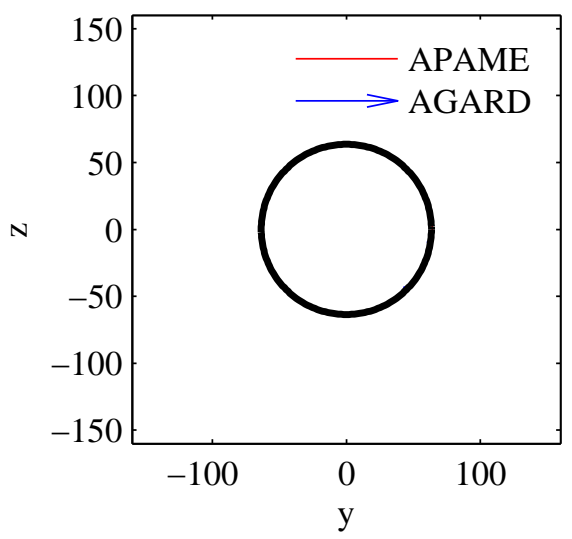

(a) $\mathrm{x}=10 \mathrm{~mm}$

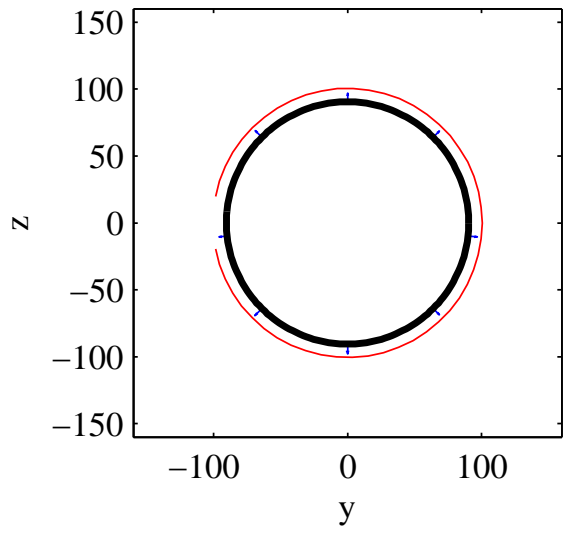

(c) $\mathrm{x}=162 \mathrm{~mm}$

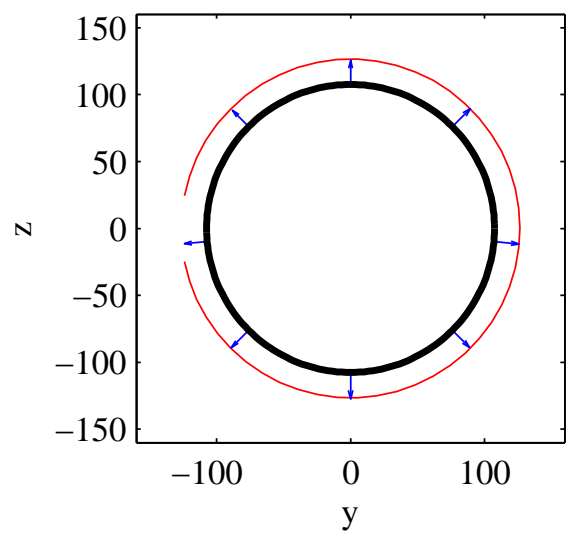

(e) $\mathrm{x}=314 \mathrm{~mm}$

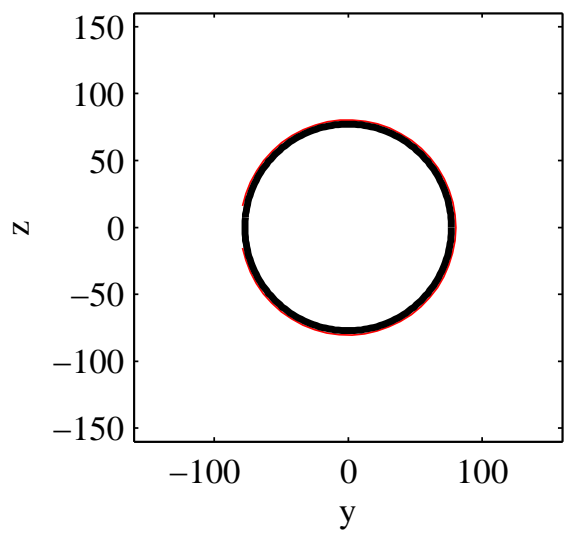

(b) $\mathrm{x}=86 \mathrm{~mm}$

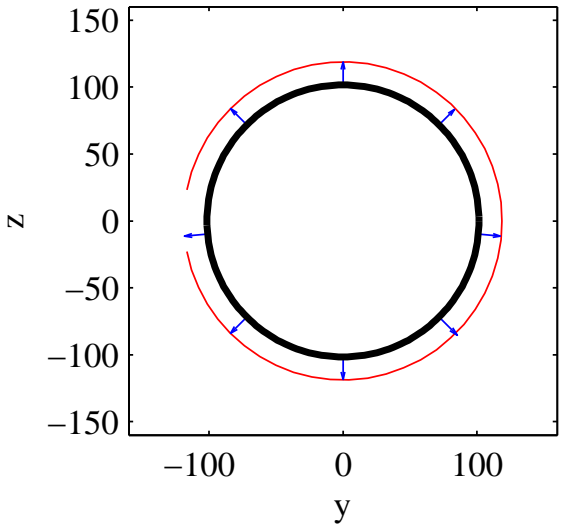

(d) $\mathrm{x}=238 \mathrm{~mm}$

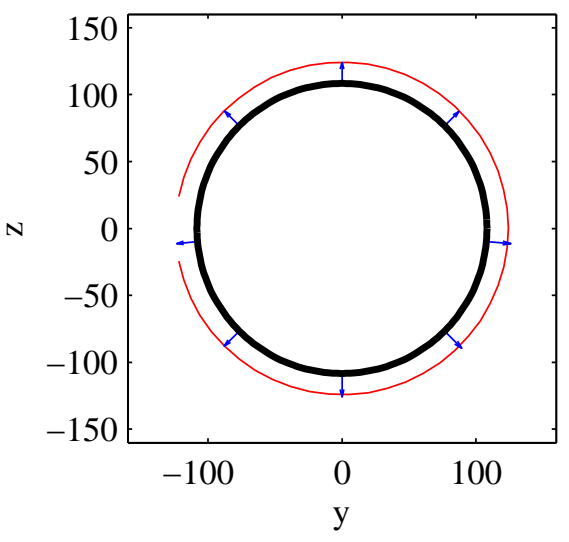

(f) $\mathrm{x}=390 \mathrm{~mm}$

Figure 9.4: Comparison of Configuration 1 experimentally measured pressure coefficients at nacelle pressure ports with computationally calculated values from the APAME panel code. 


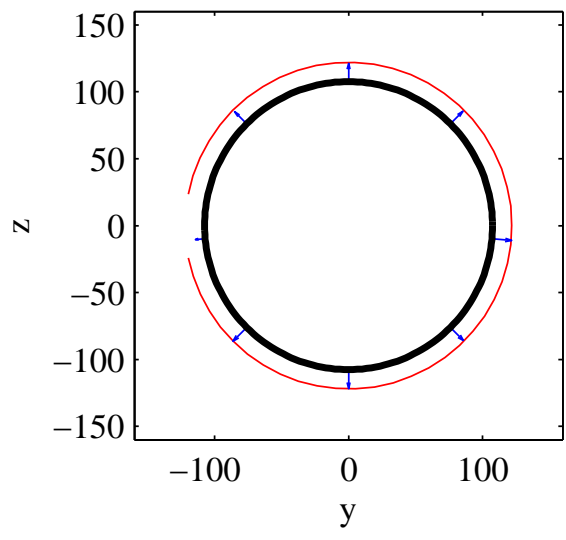

(a) $\mathrm{x}=425 \mathrm{~mm}$

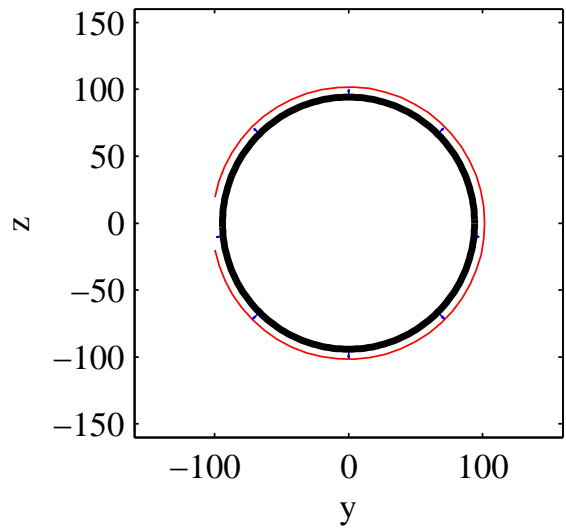

(c) $\mathrm{x}=612.5 \mathrm{~mm}$

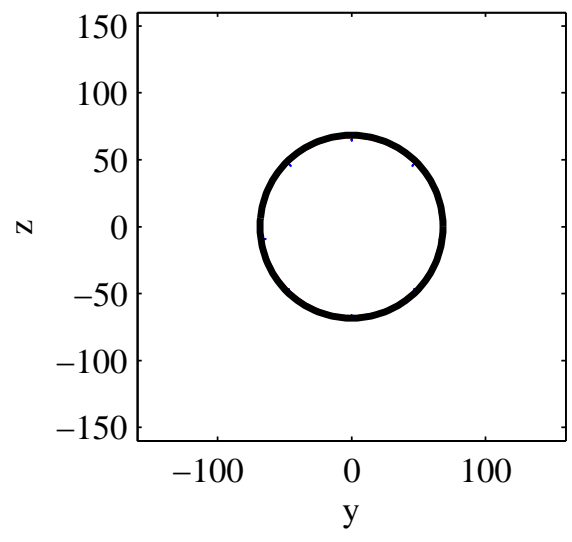

(e) $\mathrm{x}=812.5 \mathrm{~mm}$

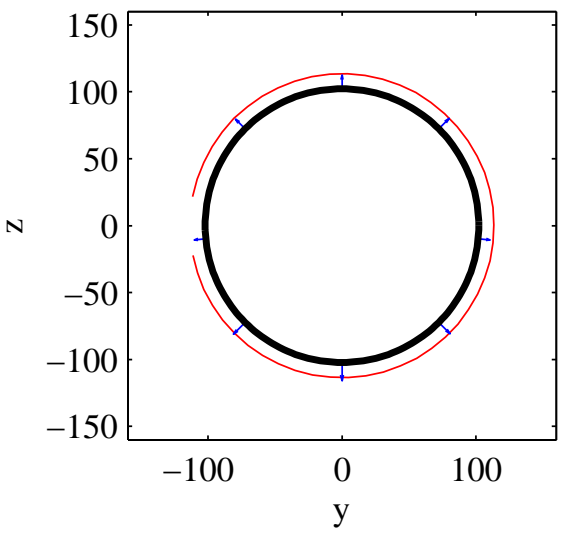

(b) $\mathrm{x}=525 \mathrm{~mm}$

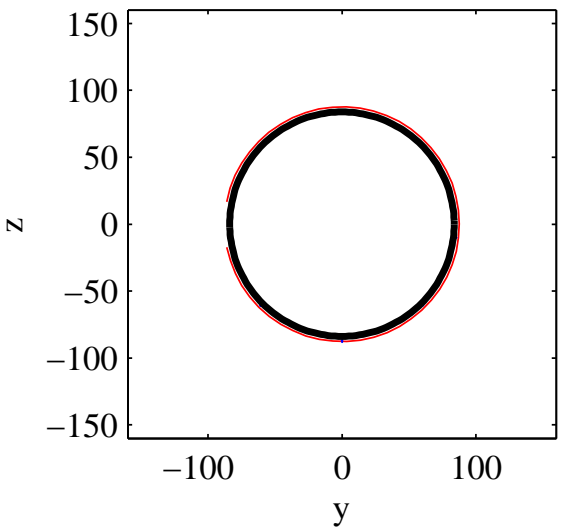

(d) $\mathrm{x}=700 \mathrm{~mm}$

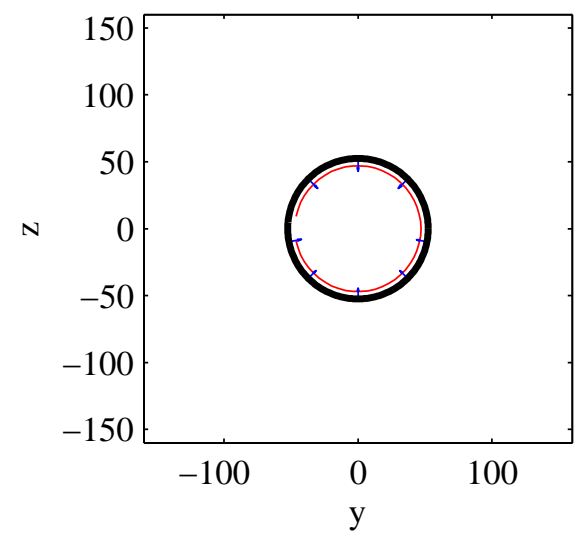

(f) $\mathrm{x}=925 \mathrm{~mm}$

Figure 9.5: Comparison of Configuration 1 experimentally measured pressure coefficients at nacelle pressure ports with computationally calculated values from the APAME panel code. 
lower on the second configuration due to the total number of panels being placed on it, but the clustering occurs in the same areas. The second configuration has a $40 \times 12$ axial-radial distribution of panels on the nacelle, with a $53 \times 17$ chordwise-spanwise panel distribution clustered at the leading and trailing edges on each halfspan, for a total of 2282 panels on it.

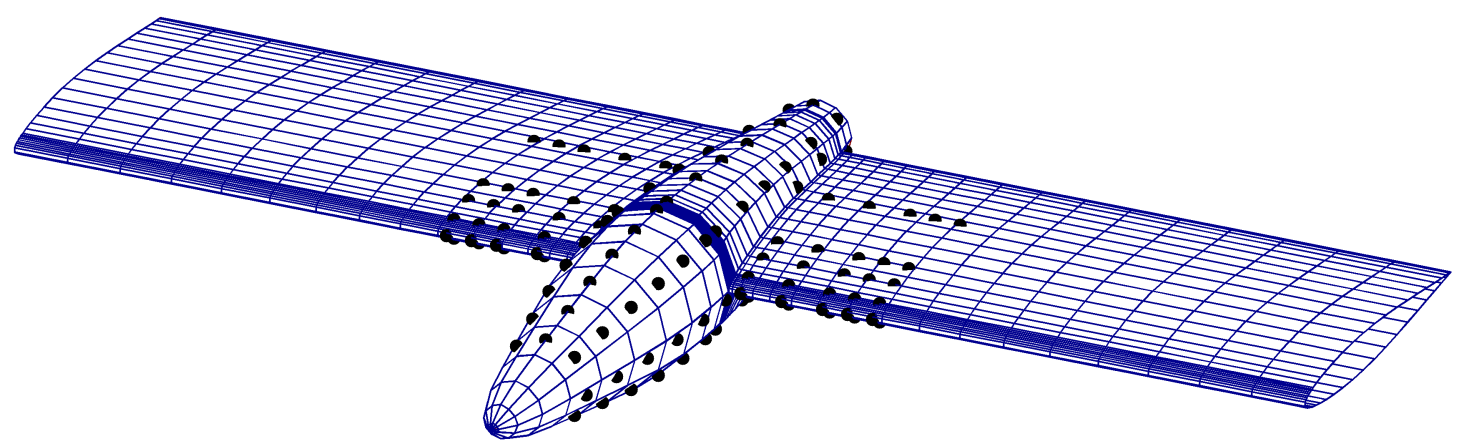

Figure 9.6: Paneled model of AGARD Configuration 2 with pressure port locations shown as black dots.

\subsubsection{Surface Pressure Coefficient Measurements}

The propeller is again off for these measurements in the wind tunnel, and so the particle scheme is not used in generating the panel code results. The same pressure coefficient scaling system employed on the first configuration is again used here, with vectors scaled by a factor of 100 from the true $C_{p}$ values. Figure 9.7 and Figure 9.8 show the pressure coefficient measurements on Configuration 2 using the same sign convention as was applied to Configuration 1. As with the first configuration, the pressure coefficient matching is excellent over the entire nacelle. In this case however, 
the matching at the final axial station of $x=925 \mathrm{~mm}$ matches poorly. It is believed that this is due to the wake panel shed from the wing, which passes extremely close to the portion of the nacelle that extends rearward beyond the trailing edge of the wing. Additionally, the trailing edge was again not paneled in this geometry, which also contributed to the error accrued at this section. 

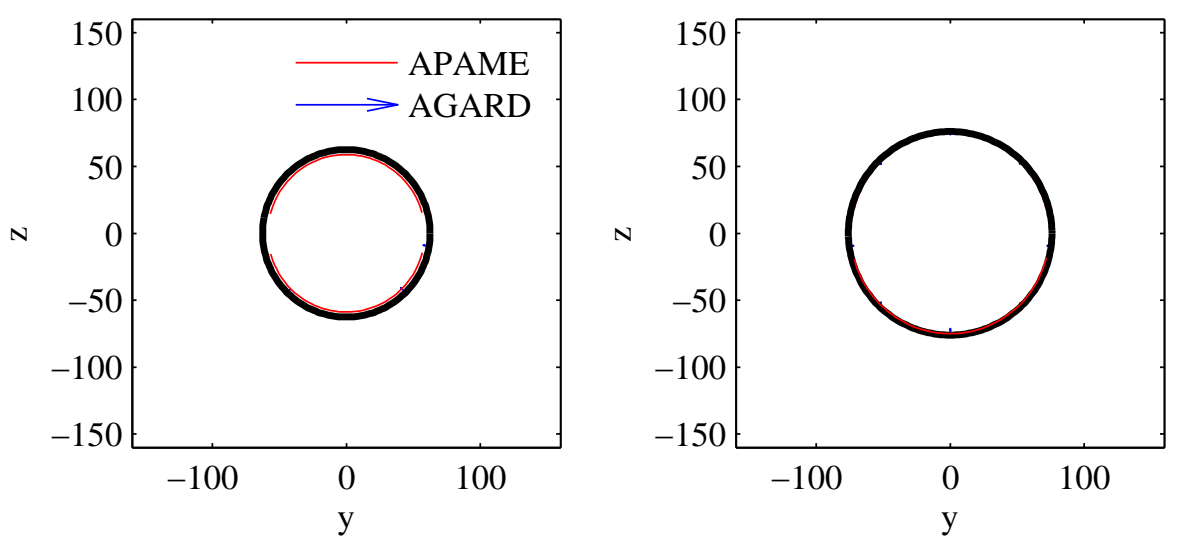

(a) $\mathrm{x}=10 \mathrm{~mm}$
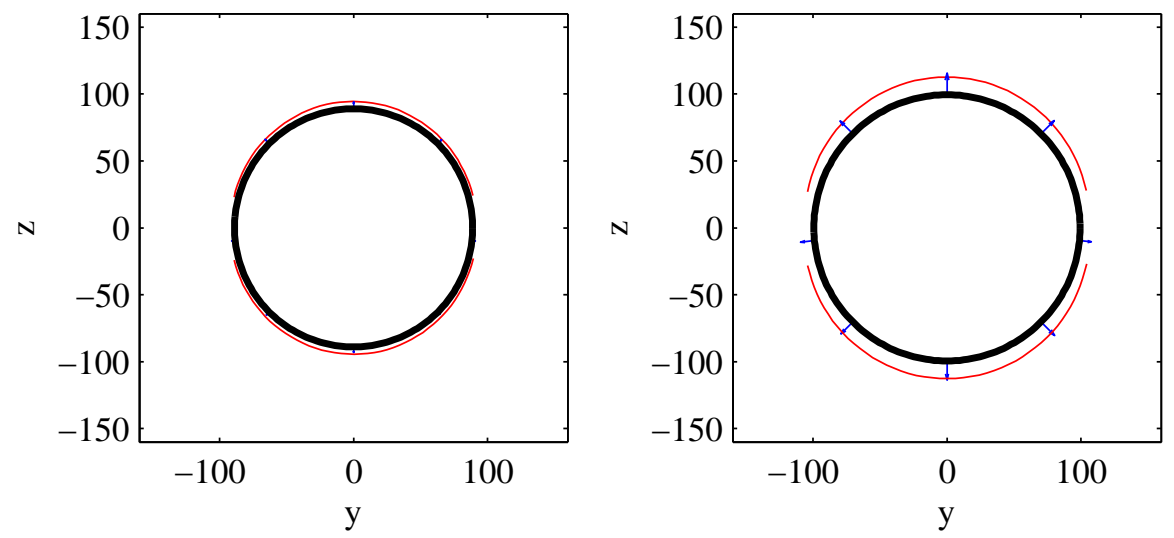

(c) $\mathrm{x}=162 \mathrm{~mm}$

(d) $\mathrm{x}=238 \mathrm{~mm}$
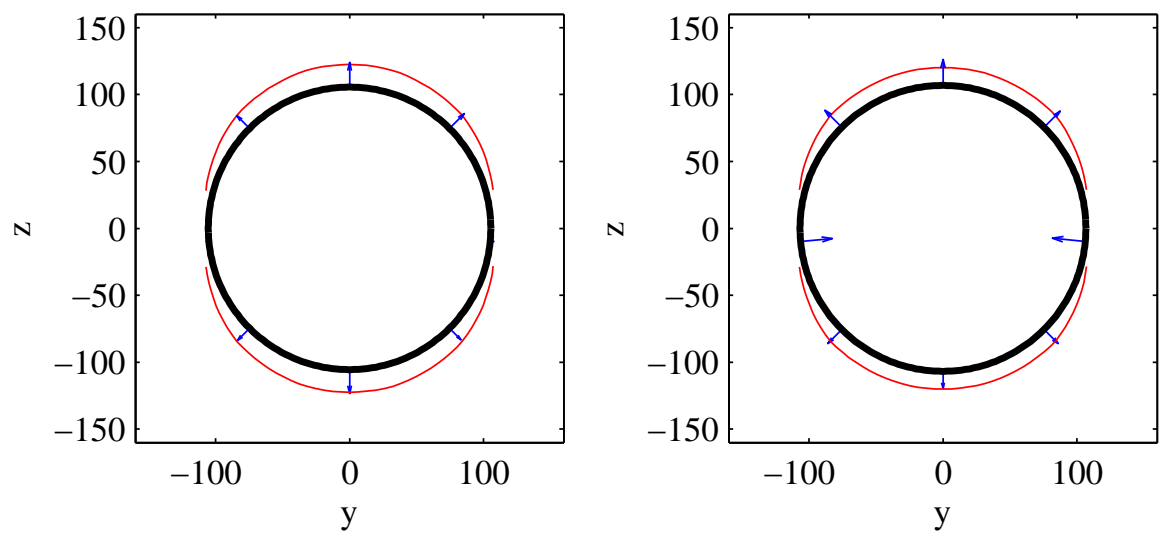

(e) $\mathrm{x}=314 \mathrm{~mm}$

(f) $\mathrm{x}=390 \mathrm{~mm}$

Figure 9.7: Comparison of Configuration 2 experimentally measured pressure coefficients at nacelle pressure ports with computationally calculated values from the APAME panel code. 


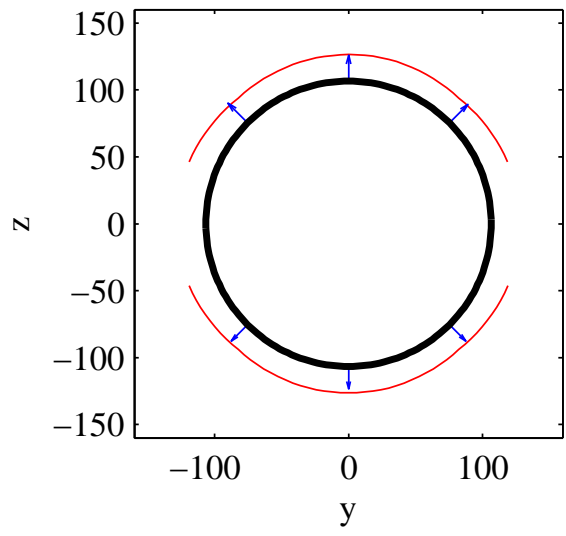

(a) $\mathrm{x}=425 \mathrm{~mm}$

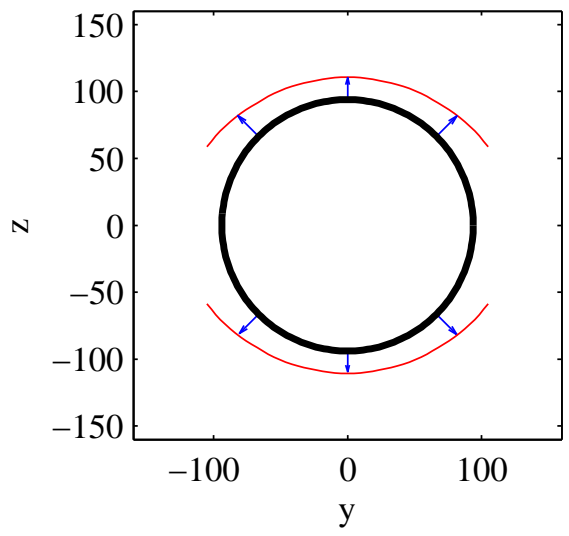

(c) $\mathrm{x}=612.5 \mathrm{~mm}$

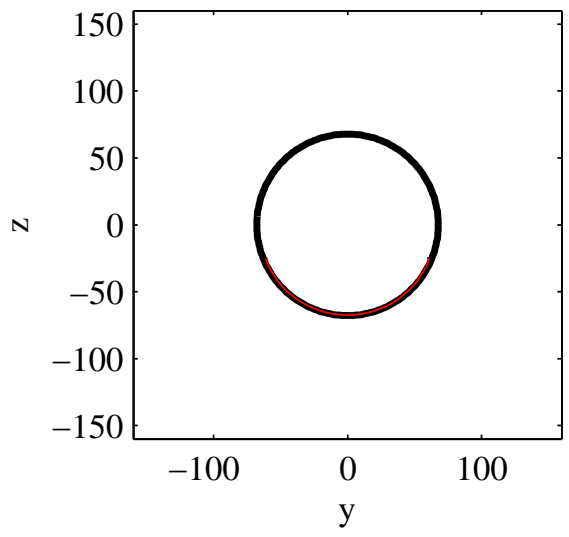

(e) $\mathrm{x}=812.5 \mathrm{~mm}$

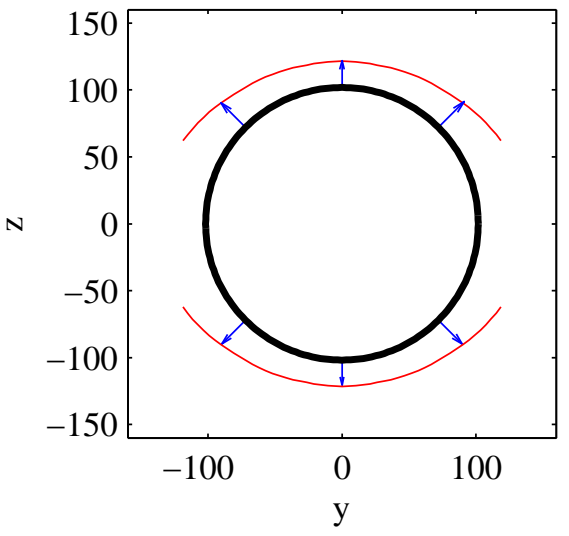

(b) $\mathrm{x}=525 \mathrm{~mm}$

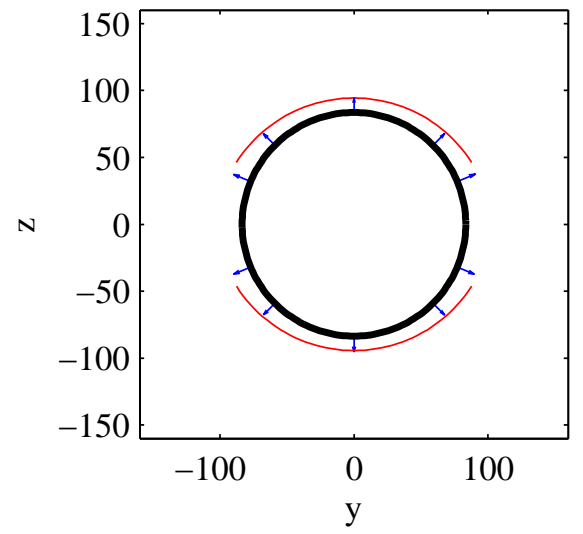

(d) $\mathrm{x}=700 \mathrm{~mm}$

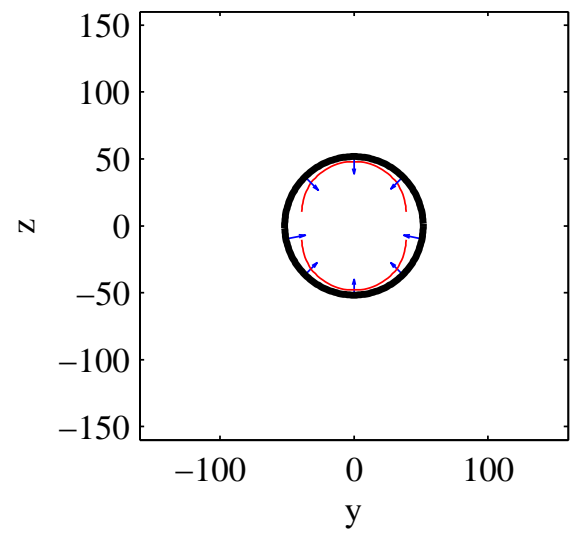

(f) $\mathrm{x}=925 \mathrm{~mm}$

Figure 9.8: Comparison of Configuration 2 experimentally measured pressure coefficients at nacelle pressure ports with computationally calculated values from the APAME panel code. 


\subsubsection{Wing Panel Density Study}

When the panel code and particle method have been combined, the number of panels present contribute to the computational expense of a given geometry in addition to the cost of the panels. A traditional vortex particle method is an $N$-body problem, and as such, the computational cost increases with an $\mathscr{O}\left(N^{2}\right)$, relationship. Because in this method all panels influence all particles, and all particles influence all panels, the computational cost of the $N$-body problem increases as $\mathscr{O}\left((M+N)^{2}\right)$, where $N$ is still the number of particles and $M$ is the number of panels representing the geometry. This cost is in addition to the cost of the matrix inversion, which tends to be insignificant relative to all of the calculations carried out in the $N$-body portion of the method. As such, it is important to truly understand the necessary number of panels to accurately represent a geometry, while at the same time not having an excess of panels that would result in unnecessary computational cost.

To find what panel density provides the ideal compromise of accuracy and computational efficiency, a series of different density grids were constructed and compared. Each of the grids was built in the meshing program ICEM through a similar process, and each from the same PRO/E model based on the geometries tested in the AGARD wind tunnel experiment. The model examined for the panel density study was the second in the series of four configurations from the AGARD paper. 


\section{Mesh Construction}

The initial model of the geometry was constructed in $\mathrm{PRO} / \mathrm{E}$, using the basic coordinates set forth in the paper detailing the wind tunnel experiment. In the paper there are coordinates that describe the airfoil coordinates and the wing dimensions, as well as a line defining the contour of the nacelle, which was revolved to produce the complete geometry. Although the airfoil coordinates were provided, there weren't an abundance of points, so the same airfoil was found in the UIUC database, and the additional points were used to provide the best discretization of the airfoil section. Once the geometry was completed, it was exported into a STEP file, which could then be read into meshing programs. Figure 9.9 shows the PRO/E model for Configuration 2 of the AGARD experiment.

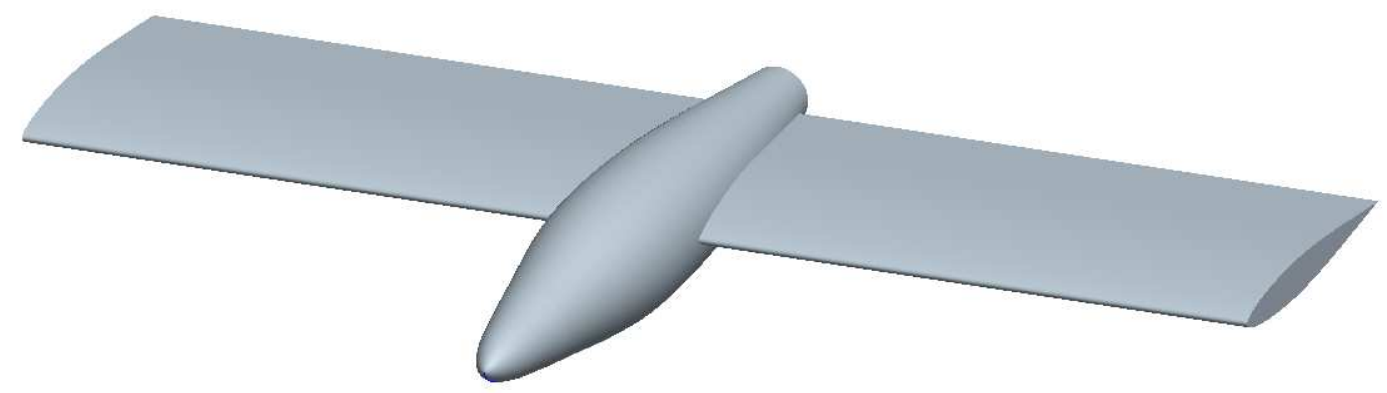

Figure 9.9: Configuration 2 model created in PRO/E 9

The STEP file was imported into ICEM, at which time the large number of parts that made up the input geometry were consolidated into two parts called WING and 
NACELLE. The surfaces that made up the wing tips were deleted so no panels would be placed there. Additionally, a BODY was placed inside the geometry so that panels would not be grown there. At this point the geometry adjustments were complete and the geometry was saved.

Once the geometry had been adjusted as necessary in ICEM, the next step was to completely block the geometry. This was done by placing a bounding block around the total geometry and then splitting that block into smaller blocks at different parts of the geometry. The block was split at each location where unique panel clustering was desired. The first block splitting was to isolate the wings from the nacelle, and this split the block at the outermost point on each side of the nacelle where the nacelle and wing intersected. The block was additionally split along the leading edge and trailing edge of the nacelle. At this point it was possible to delete four of the split blocks due to the fact that they contained no geometry, and those four blocks were located on each span side both directly in front of and behind the wing.

Once the initial cuts were made, and the extraneous blocks removed, some additional splits were made to further refine the geometry separation. The nacelle was split both horizontally and vertically down its axis, with the horizontal cut also splitting the wing upper and lower surfaces. Additionally, a cut was made from wing tip to wing tip at around $5 \%$ chord to provide high panel counts for leading edge refinement in that region, and this cut also affected the nacelle geometry. Once the blocking was completed, there were a total of 24 different patches that had been separated by 
cuts. Each half span had a leading edge section and a section that contained the rest of the airfoil for both the upper and lower surface, resulting in 8 total wing patches. The nacelle was split chordwise into a section before the leading edge of the wing, a section where the leading edge refinement cut was made, a section containing the rest of the wing, and a section with a small nacelle piece that came after the wing. Each of these sections has four patches in it due to the fact that the nacelle was split axisymmetrically into equal quarters, resulting in an additional 16 patches, for a total of 24. After all of the blocks had been created, some association of the various block edges and vertices was required.

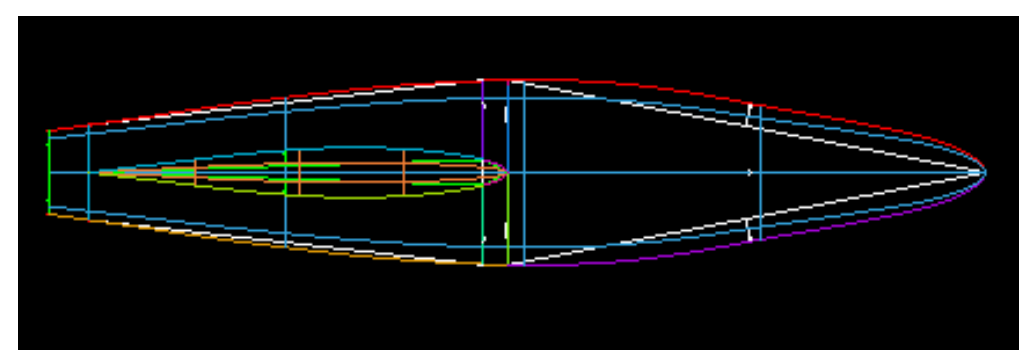

Figure 9.10: Side view of edge association in ICEM.

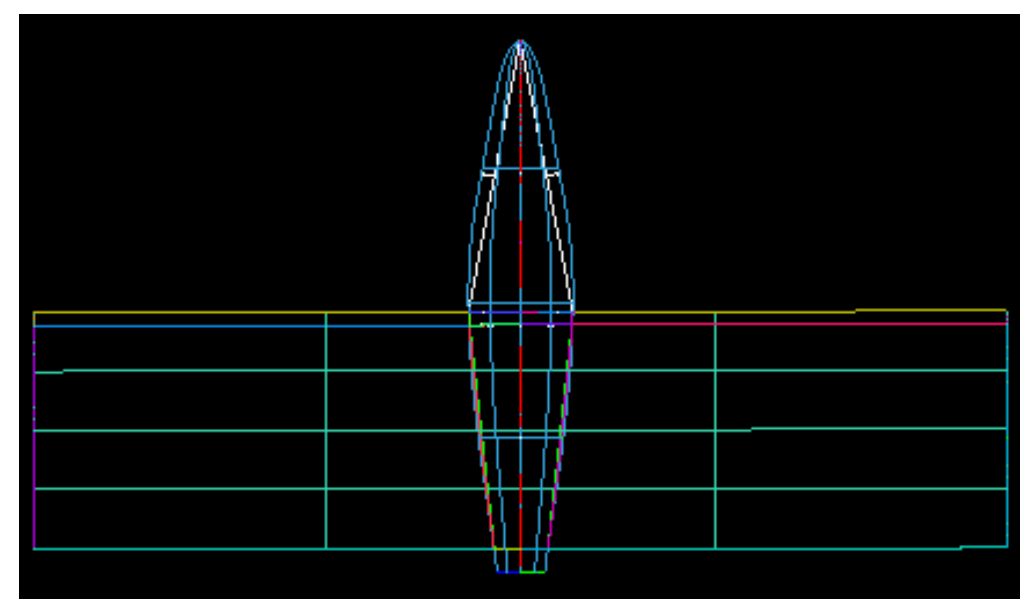

Figure 9.11: Top view of edge association in ICEM. 


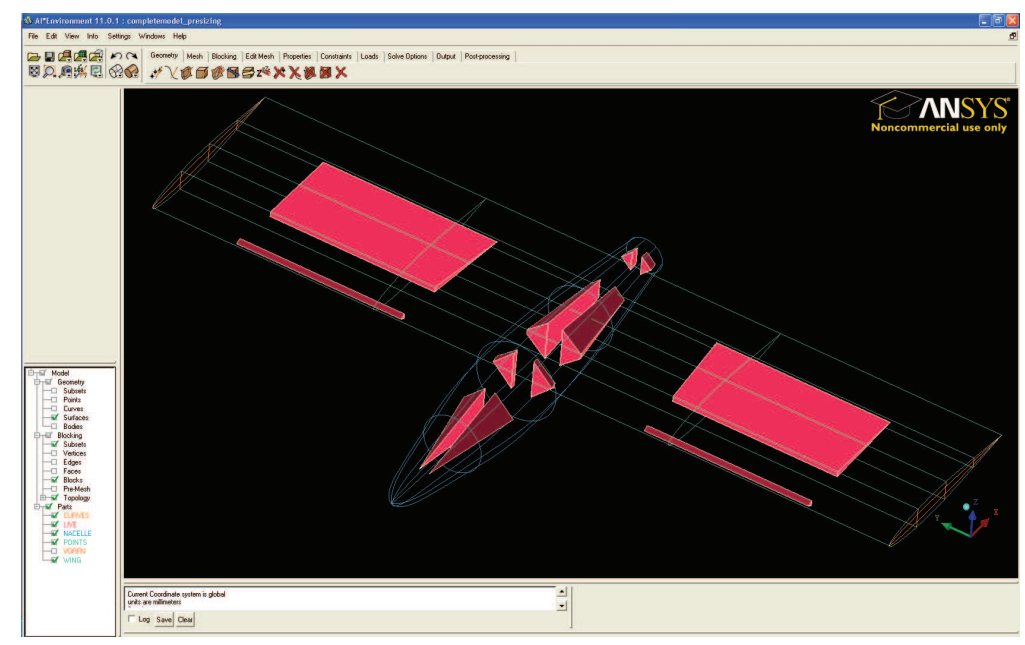

Figure 9.12: Iso view of separate blocks in geometry in ICEM, scaled to show clear separation between blocks.

To fine tune exactly how the panels were distributed it was necessary to apply edge meshing parameters. Using the edge mesh parameters it was possible to specify a desired number of nodes, as well as a distribution with which to apply those nodes to the geometry. The AGARD wind tunnel test, to which this method is being compared, examines pressure data in and around the streamtube of the propeller, so it was important to have a high density of panels in the region that the streamtube would most affect. As such, panels were clustered towards the nacelle on the wings using half cosine distributions. The inner most panel was set at 5 millimeter width, and each successive cell was grown in size from that. A full cosine distribution was used on the chord of the main section of the wing, ensuring that there would be clustering towards both the leading and trailing edges of the airfoil. The portion of the nacelle in front of the wing was set using the bigeometric spacing, to provide higher densities at the nose, as well as near the beginning of the wing-nacelle interface, 
where large gradients were expected. The leading edge section of the wing received a high number of cells because of the high amount of curvature in the geometry at that location. The section of the nacelle behind the wing wasn't terribly large, but it was important to have at least two chordwise panels there to allow for finite differences to be taken so that the panel code could generate a solution.

\section{Mesh Density Comparison}

To obtain the panel density that provides the right balance between accuracy and computational cost it was necessary to compare a variety of different grids. Each of the grids examined used the same distribution functions, but the number of nodes on the various edges in the geometry were changed in each case. The different panel densities were compared through examination of the pressure coefficient at the spanwise locations on the wings at which the AGARD wind tunnel test places pressure taps. Because there was no way of knowing where panel collocation points would be relative to the pressure port locations, the data at each collocation point for each density was placed into an interpolation function in MATLAB called griddata. The pressure coefficient was taken at 60 chordwise locations to provide a reasonable data set to plot.

Geometries with 382, 762, 1882, 2746, and 4252 panels were all analyzed, and the pressure coefficient slices were produced for each. Examination of any of the different spanwise locations showed that, while the 382 and 762 panel schemes were noticeably different from the other panel densities, the higher panel counts all behaved quite 
similarly, except at the leading edge. The two highest panel count cases had a higher number of panels in the leading edge refinement region for which a unique block was created.

\section{Final Mesh Selection}

The final panel density selected was the 1882 panel geometry, modified to have the same leading edge panel density as the two higher panel cases. This resulted in a total of 2282 panels. This panel count was deemed to capture the true airfoil characteristics enough, while at the same time maintaining a somewhat low panel count to reduce the $\mathscr{O}\left((M+N)^{2}\right)$ computational costs.

Those measurement values are presented in Figure 9.13. The pressure coefficients from the experimental data over the surface of the wing at various spanwise stations are denoted by markers, while the resultant pressure coefficients from the panel code are plotted as a distribution for both the upper and lower surface. Because the distance measurements are normalized by the chord length, the pressure coefficient values are not scaled in Figure 9.13. There is some clear pressure difference between the upper and lower surfaces of the experimental data, indicated by the fact that the experimental markers in the plots do not completely overlap. These effects are not captured in the panel code results, and whatever the source of the errors from the wind tunnel, the same effects are likely present in all of the experimental measurements. 


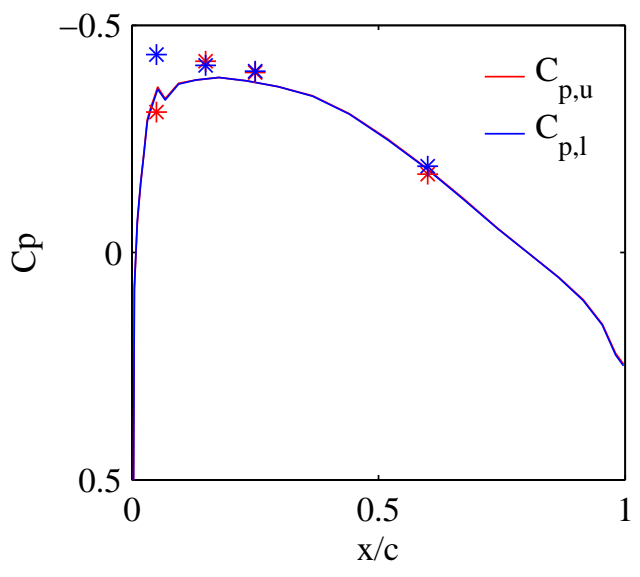

(a) $\mathrm{y}=130 \mathrm{~mm}$

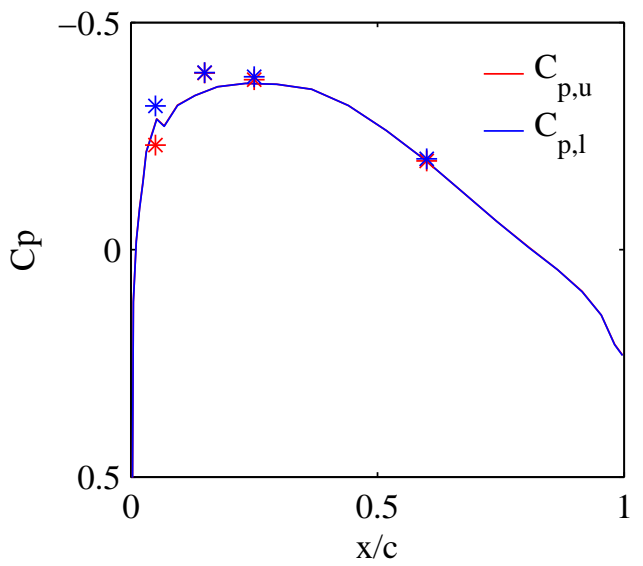

(c) $\mathrm{y}=260 \mathrm{~mm}$

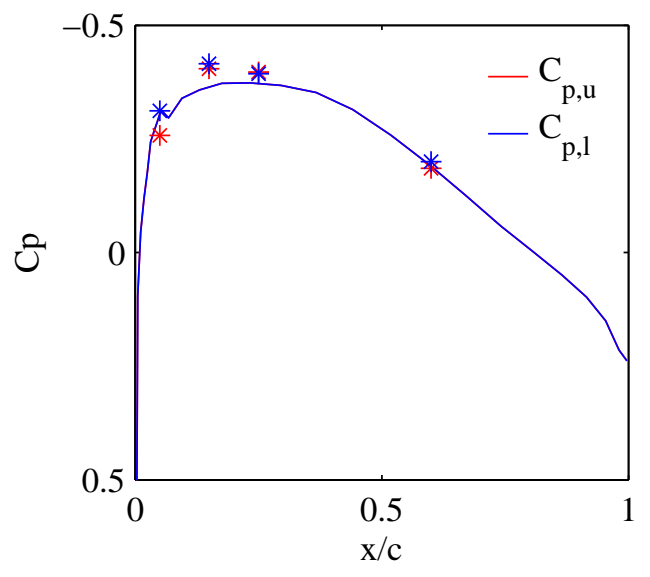

(b) $\mathrm{y}=195 \mathrm{~mm}$

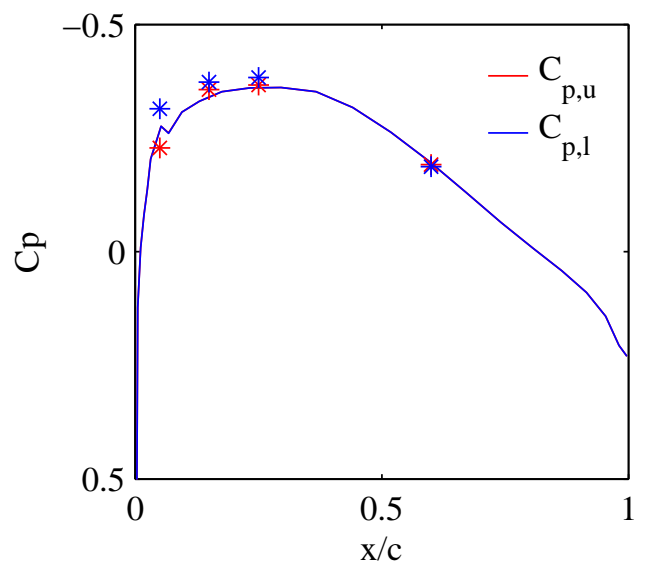

(d) $\mathrm{y}=300 \mathrm{~mm}$

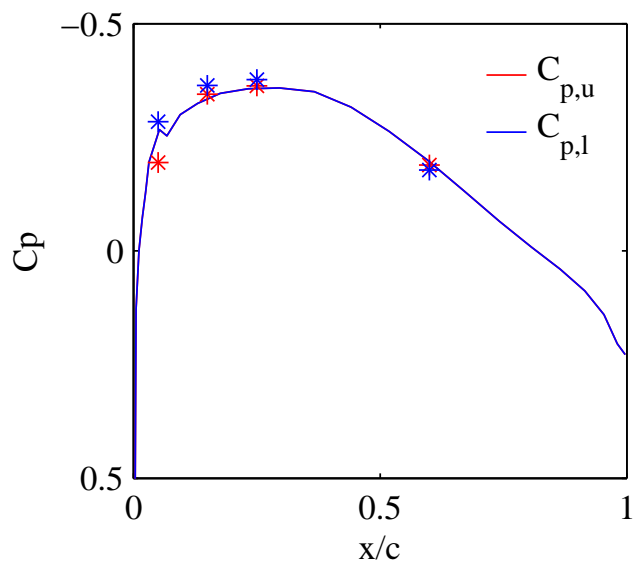

(e) $\mathrm{y}=340 \mathrm{~mm}$

Figure 9.13: Comparison of Configuration 2 experimentally measured pressure coefficients $(*)$ at wing pressure ports with computationally calculated values (-) from the APAME panel code. 


\section{Chapter 10}

\section{Propeller-Airframe Interaction Simulations}

With a complete method constructed whose individual components had been validated, the final step was to test the validity of the method in double precision. The AGARD wind tunnel tests provided a great benchmark to use for comparison, and so investigation of the merits of the method began there. Configuration 1 of the AGARD experiment was used as a calibration tool to ensure that particle strengths were correctly assigned to obtain the desired axial and swirl velocity components. Once desired strengths were known, those strengths were then transferred to the Configuration 2 model to obtain results in a more complex flowfield. Finally, as a further case study of interest, a channel wing was examined with a propeller similar to that of the AGARD calculations. 


\subsection{Propeller Distribution Matching}

One of the steps required to most exactly match the AGARD wind tunnel data was to find the best process to match the propeller flowfield using the vortex particle actuator disk. Matching the propeller required matching of a variety of propeller characteristics, including dimensions, velocity distributions, swirl effects, and thrust coefficient.

\subsubsection{Axial Velocity Distribution Matching}

To match the axial velocity distributions in the streamtube, it was necessary to have a desired velocity profile as a starting point. The profile should correspond to the desired axial perturbation velocity distribution far downstream, although this can be simplified to the distribution at the disk. The AGARD experimental results provided five hole probe survey data directly behind the disk, so the distribution over the blade length of the propeller was input into MATLAB. The particle strengths could then be set using Equation 6.1, which is repeated here for convenience.

$$
\frac{d V_{z}(r, 0)}{d r}=-\frac{\gamma(r)}{2} .
$$

While applying the above method provides the correct shape of the axial velocity distribution, it does nothing to scale that strength. Therefore, to get the right magnitude of the distribution, the profile that was selected to be differentiated must be scaled. As mentioned previously, Appendix B provides the details of one possible scheme that was created to do this. For the AGARD validations, the particle strengths 
were tweaked to provide the best possible approximation of the experimental velocity profiles at $x=10 \mathrm{~mm}$ and $x=525 \mathrm{~mm}$.

\subsubsection{Swirl Velocity Distribution Matching}

While the actuator disk theory of Conway provides a small amount of insight into the application of swirl velocity, $V_{\phi}$, nearly all computational simulations assumed zero $V_{\phi}$. Upon further investigation of the proposed method based around the vortex system of Equation 5.12 and Equation 5.13 recommended by Conway, the resultant swirl distribution was not reflective of a real propeller. As with the axial velocity distribution, the AGARD experimental data was examined to gain insight into the true flowfield swirl properties associated with time averaged measurements of a propeller.

Configuration 1 provided the best insight into the swirl distribution because of a lack of wing downstream to interact with the flow, and so the data from that particular test was examined most closely. The data showed a parabolic $V_{\phi}$ profile inside of the streamtube, and negligible $V_{\phi}$ outside. Because the solution based in actuator disk theory with hub and tip vorticity only was deemed unrealistic for true propeller flows, the first solution tried was a simple linear distribution of vorticity applied across the disk. A special condition was placed on the vorticity, which was that over the disk area there was zero net vorticity. This condition made a conservative system, and therefore vorticity was theoretically not being created or destroyed. This linear distribution proved to work reasonably well at capturing the overall swirl distribution, and much like the axial distribution, could be easily scaled to match values from the AGARD 
experiment.

\section{No Net Axial Vorticity Derivation}

To conserve axial vorticity in the field there must be no net axial vorticity when the particle strengths are assigned to create swirl velocity. The strengths can be set with a linear distribution that provides zero net vorticity by assuming an arbitrary linear function $A+B r$ between the hub radius and the tip radius. The integral of that linear function over the actuator disk is then set equal to zero and the slope is solved for.

$$
\int_{R_{h}}^{R_{a}} \int_{0}^{2 \pi}(A+B r) r d r d \theta=0 .
$$

The integral of the sum can be broken apart into two separate integrals resulting in

$$
\int_{R_{h}}^{R_{a}} \int_{0}^{2 \pi} A r d r d \theta+\int_{R_{h}}^{R_{a}} \int_{0}^{2 \pi} B r^{2} d r d \theta=0 .
$$

Integrating first with respect to $\theta$ results in

$$
2 \pi\left(\int_{R_{h}}^{R_{a}} A r d r+\int_{R_{h}}^{R_{a}} B r^{2} d r\right)=0 .
$$

Next the radial integration can be carried out, resulting in

$$
\frac{A}{2}\left(R_{a}^{2}-R_{h}^{2}\right)+\frac{B}{3}\left(R_{a}^{3}-R_{h}^{3}\right)=0
$$

Equation 10.4 can then be solved for $B$ to define the function that provides zero net vorticity.

$$
B=-\frac{3 A}{2} \frac{R_{a}^{2}-R_{h}^{2}}{R_{a}^{3}-R_{h}^{3}} r
$$


Thus, axial vorticity required for swirl flow can be assigned as

$$
\omega_{x}=A-\frac{3 A}{2} \frac{R_{a}^{2}-R_{h}^{2}}{R_{a}^{3}-R_{h}^{3}} r
$$

where $A$ is a scaling factor to determine the magnitude of the imparted swirl velocity.

\subsection{AGARD Configuration 1}

The first configuration examined in detail with the particle-panel code was Configuration 1 of the AGARD experiment. Due to its simplicity relative of the other configurations, Configuration 1 was deemed ideal as a calibration tool to make sure that particle strengths were being set in a way most representative of the true physics that occurred in the experiment. The scaling factors of the strengths were adjusted in an ad-hoc manner to achieve good pressure coefficient matching over the entire surface of the nacelle. The simulation was run with the particle strength evolution both on and off. When the strength evolution was off, the particle strengths were not updated, and the right hand side of Equation 3.39 was set equal to zero. The presence of stretching in the start up wake led to instabilities, so the main results shown here are with the strength evolution off.

\subsubsection{Resultant Pseudo-Steady Flowfield}

The propeller was simulated with an actuator disk discretized into $N_{s}=112$ particles released per time step. The specified convection distance was approximately 6 propeller radii downstream, which equates to about 2 nacelle chord lengths. The 
specified convection length resulted in a core radius of $44.6 \mathrm{~mm}$ and time step size of 0.0012 seconds, requiring 33 time steps to complete the convection. The viscosity of the viscous diffusion model was set based on characteristics from the wind tunnel experiment to be $1.4144 e^{-5} \frac{m^{2}}{s}$. The final particle locations are shown in Figure 10.1 . The wake has been convected a suitable distance downstream so that there is little concern of it significantly influencing the geometry or particles around the nacelle, where the suitable distance was determined through experience running cases with various convection distances.

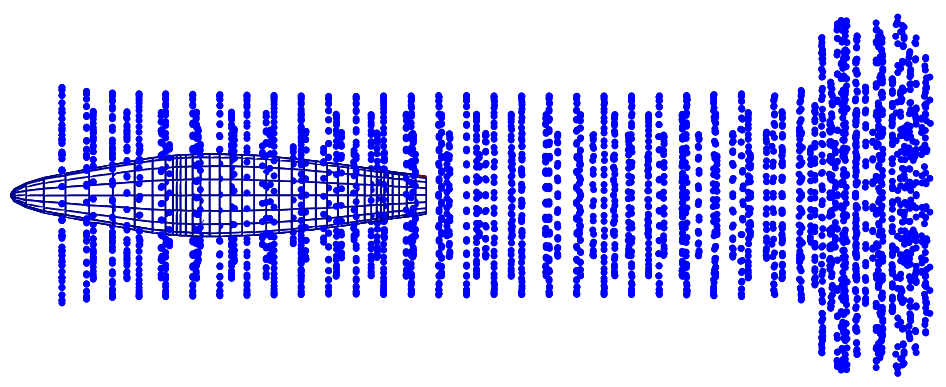

Figure 10.1: Fully convected particle locations around Configuration 1 at the end of 33 time steps.

Over the course of the simulation, the aerodynamic coefficients are calculated and stored at the conclusion of each time step. The coefficients can then be examined at the end of the simulation, regardless of whether the case was run with stretching or not. The results at step 0 are the coefficients for the geometry alone, before any particles have been introduced. As would be expected for an axisymmetric nacelle, there are no moment coefficients, and the only nonzero force coefficient is the drag, which is still on the order of $10 e^{-3}$, and likely stems from numerical error. When stretching is included, the initial portion of the simulation follows the same trends as 
without stretching. All coefficients except drag remain zero, and the drag coefficient is still quite small. Around the twentieth time step however, instability occurs and the solution breaks down. This is believed to be a function of the stretching and the start up wake. As the time step number increases, more and more particles become entrained in the wake, which is an area of extremely high velocity gradient. The high velocity gradient directly factors in to the adjustment of particle strengths, and so particles in the wake tend to have dramatically increased strengths. The increase in strengths magnifies numerical errors and asymmetry in the particle discretization and panel distribution, causing the simulation to fail. The coefficient values with and without stretching are shown in Figure 10.10 .

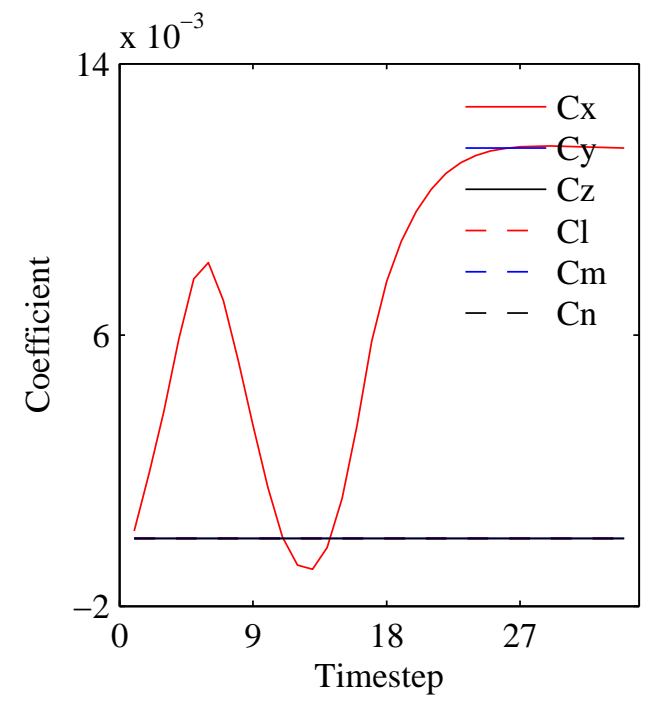

(a) No Stretching

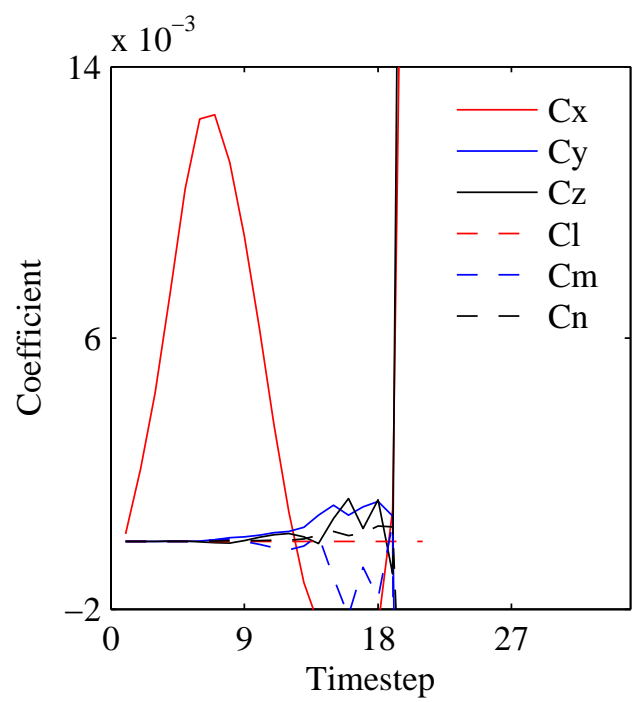

(b) With Stretching

Figure 10.2: Aerodynamic coefficients for Configuration 1 with and without stretching turned on. 


\subsubsection{Velocity Profile Comparisons}

An important component of ensuring that the particle-panel code can model propulsion-airframe effects is to see if the correct field velocity profiles are captured. This problem is more challenging than simply matching the pressure coefficient values on the surface, and the capability to capture the entire profile would be necessary to find effects on geometry in the slipstream aside from the nacelle. Figure 10.3 shows a comparison of the velocity field induced by the particles with that of the experimental data at an axial station immediately downstream of the propeller. The surface of the nacelle is denoted with the black dashed line to give perspective as to how close the survey came to the surface. The matching is adequate in magnitude, and the distribution of the numerical simulation is slightly different than that of the experimental profile. It is important to note that the five hole probe measurements were not made down to the surface of the nacelle at this axial station.

The next station at which velocity profiles were measured was at $x=525 \mathrm{~mm}$, which is about halfway down the spine of the nacelle. The magnitudes once again match well with the experimental data, and now the computational profile has evolved and is also a slightly better fit for the overall profile shape of the experimental data. The axial and swirl velocity profiles are shown in Figure 10.4 .

The final velocity profile sweep was conducted at $x=925 \mathrm{~mm}$, which is almost to the end of the nacelle. The profiles are shown in Figure 10.5 


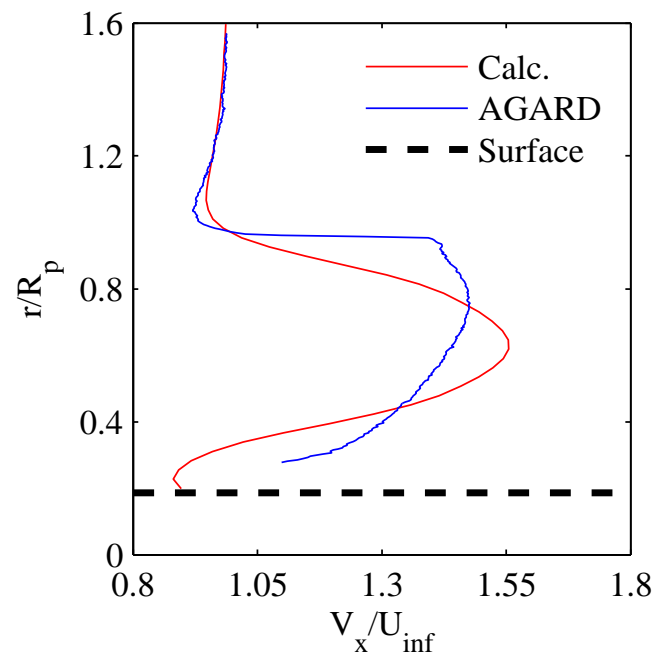

(a) Axial

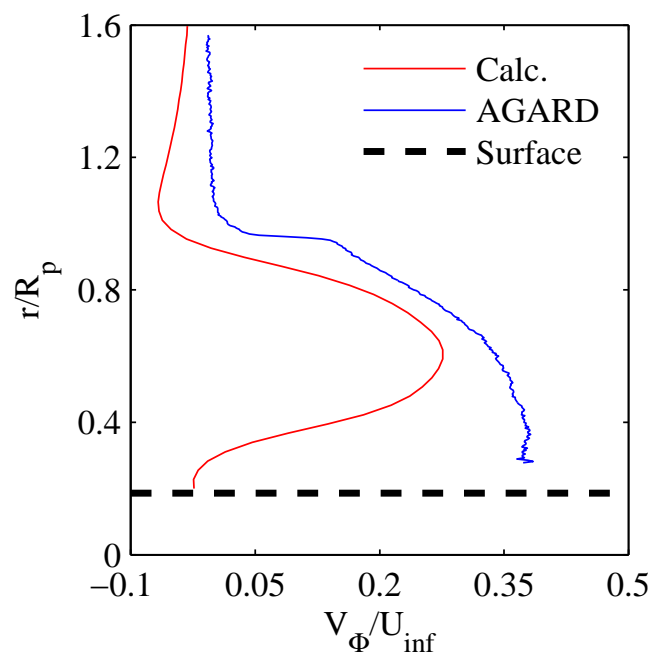

(b) Swirl

Figure 10.3: Velocity profiles at $x=10 \mathrm{~mm}$, close behind the actuator disk for Configuration 1.

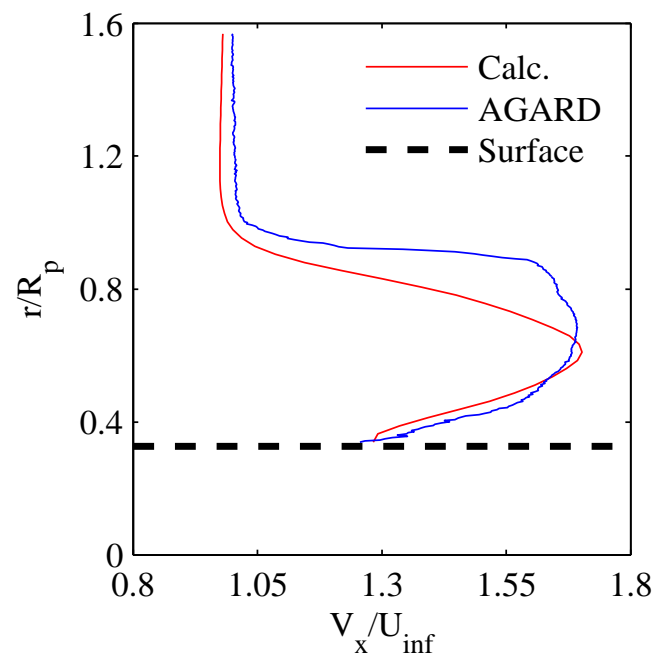

(a) Axial

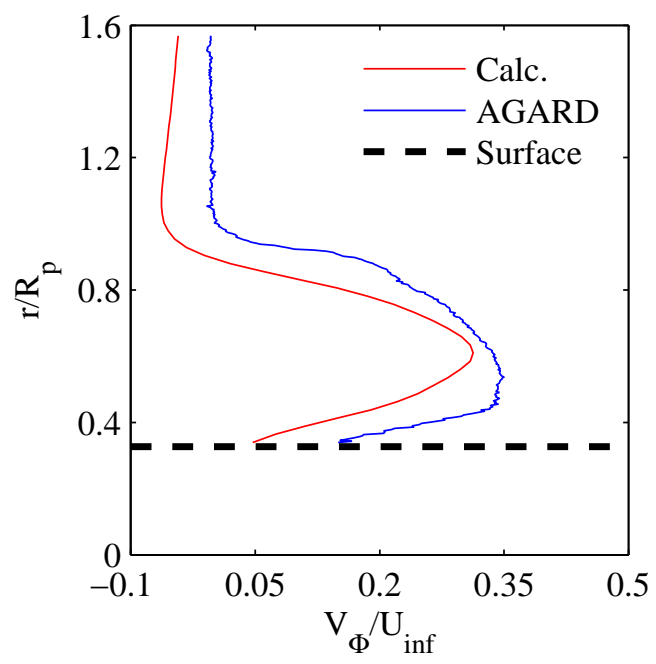

(b) Swirl

Figure 10.4: Velocity profiles at $x=525 \mathrm{~mm}$, around the middle of the nacelle for Configuration 1. 


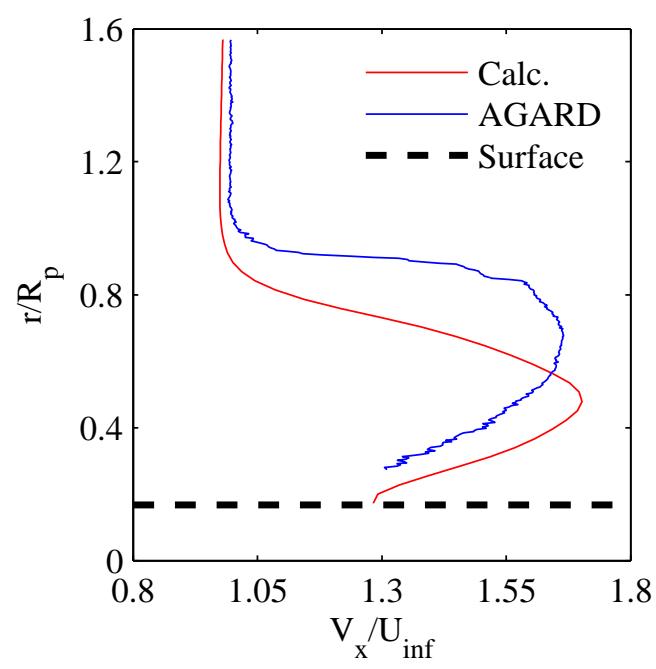

(a) Axial

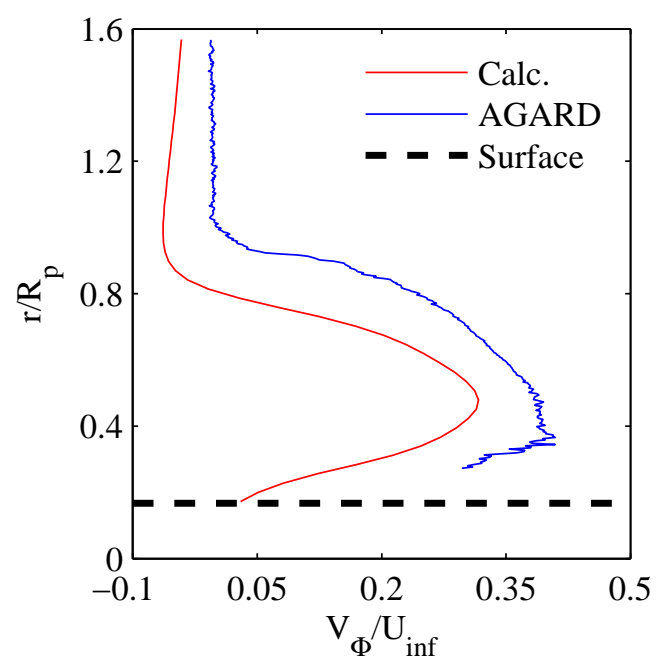

(b) Swirl

Figure 10.5: Velocity profiles at $x=925 \mathrm{~mm}$, near the end of the nacelle for Configuration 1.

\subsubsection{Nacelle Pressure Coefficients}

With confidence that the velocity profile over the geometry was reasonably well matched, and that the flowfield was well converged around the nacelle, the final step was to compare pressure coefficient values over the surface with experimental measurements. Overall the measurements are quite good, however there are certain ares of the nacelle were matching is poor. The poor matching can be explained by a small feature of the swirl velocity profile. The numerical dynamic pressure coefficients are based on the magnitude of the velocity on the surface of the nacelle.

An examination of the swirl velocity profile in Figure 10.3 shows that there is a small amount of reverse swirl flow on the surface of the nacelle. By the time the flow reaches $x=525 \mathrm{~mm}$ that reverse flow has disappeared, and the minimum value on the surface of the nacelle is approximately the same magnitude, but in the opposite 
direction. This indicates that between $x=10$ and $x=525$ the swirl velocity on the surface has gone from slightly positive in one direction to zero and then to a positive value in the opposite direction, when it likely should have stayed constant. The decrease in velocity magnitude during this period explains the slightly higher pressure coefficient values measured toward the front of the nacelle. The pressure coefficient comparisons over the whole nacelle are shown in Figure 10.6 and Figure 10.7.

\subsection{AGARD Configuration 2}

With the velocity profiles calibrated against Configuration 1 results, the same particle inputs were then carried over to Configuration 2 to see the true capability of the method. Along with the same particle strength assignment, the rest of the particle inputs, including number of particles and viscosity were left the same for Configuration 2, while the flow was convected for 31 time steps. The instabilities that arose with the stretching equation on prevented any good answers from being

generated, so all results presented for Configuration 2 are without any evolution of the initial particle strengths.

\subsubsection{Resultant Pseudo-Steady Flowfield}

The flowfield was again examined once the area around the geometry was determined to be in a pseudo-steady state. The streamtube is much more interesting with the wing present because the swirl velocity interacting with the wing causes a split in the streamtube aft of the nacelle, with the upper portion of the streamtube 


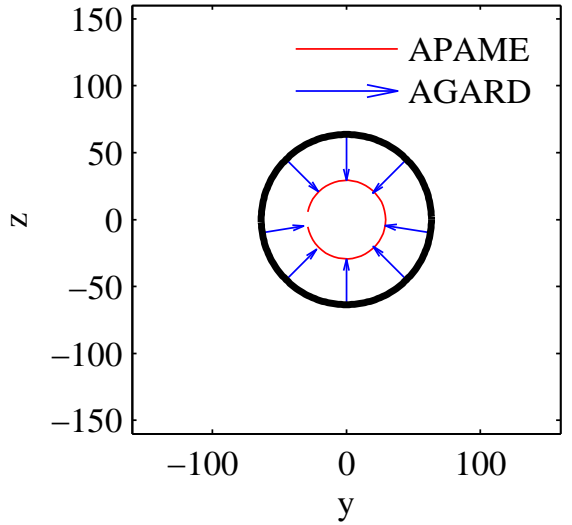

(a) $\mathrm{x}=10 \mathrm{~mm}$

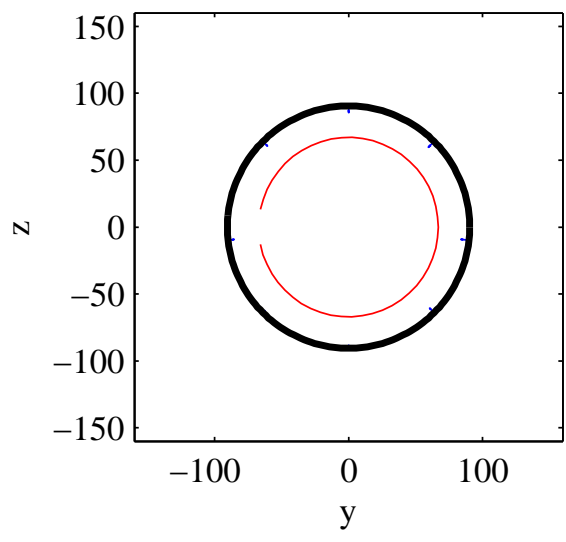

(c) $\mathrm{x}=162 \mathrm{~mm}$

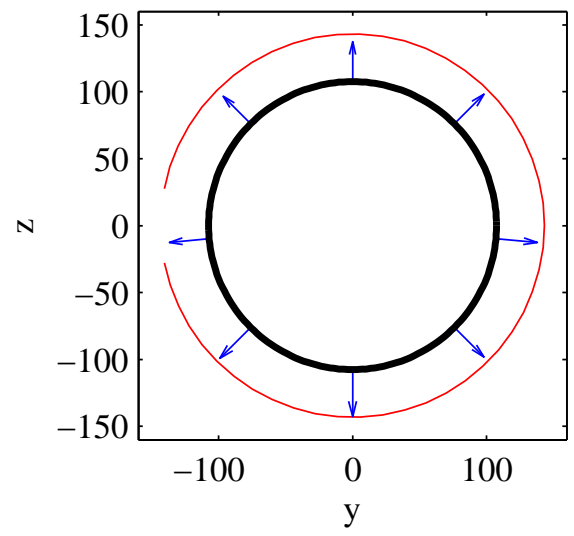

$(\mathrm{e}) \mathrm{x}=314 \mathrm{~mm}$

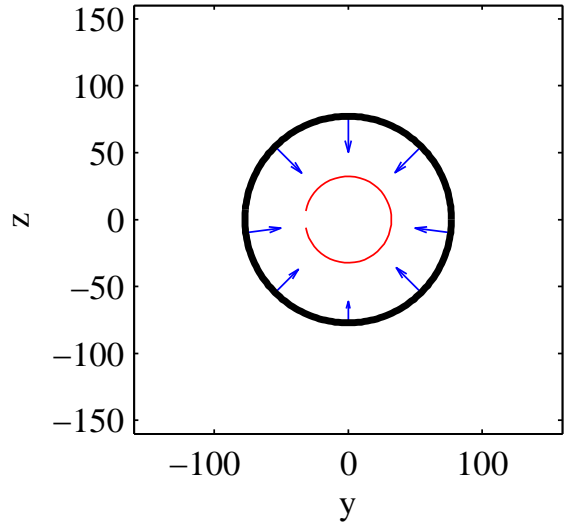

(b) $\mathrm{x}=86 \mathrm{~mm}$

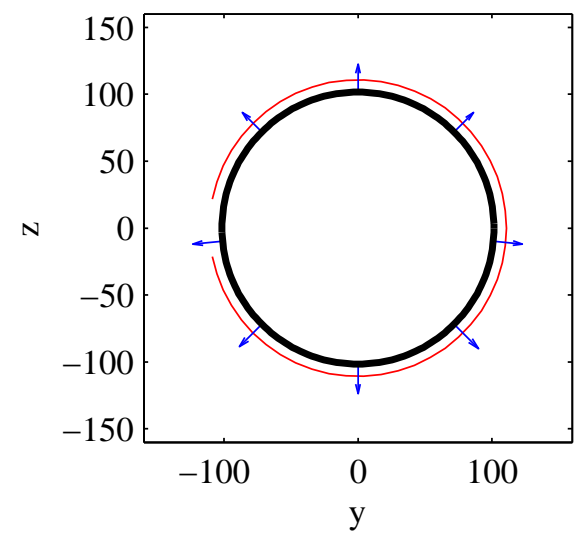

(d) $\mathrm{x}=238 \mathrm{~mm}$

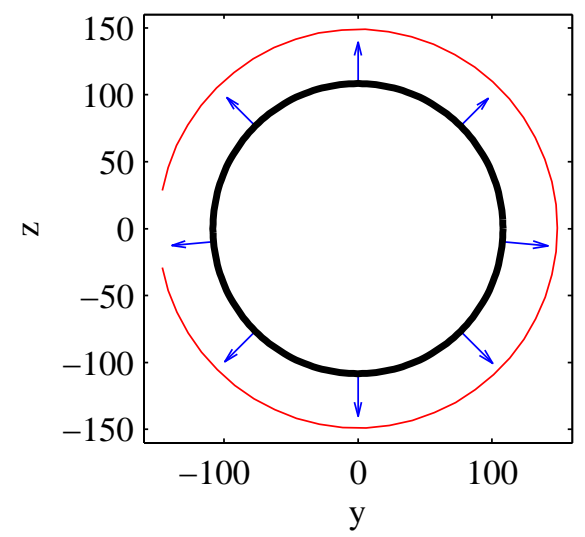

(f) $\mathrm{x}=390 \mathrm{~mm}$

Figure 10.6: Comparison of Configuration 1 experimentally measured pressure coefficients at nacelle pressure ports with computationally calculated values from the APAME panel code with the propeller on. 


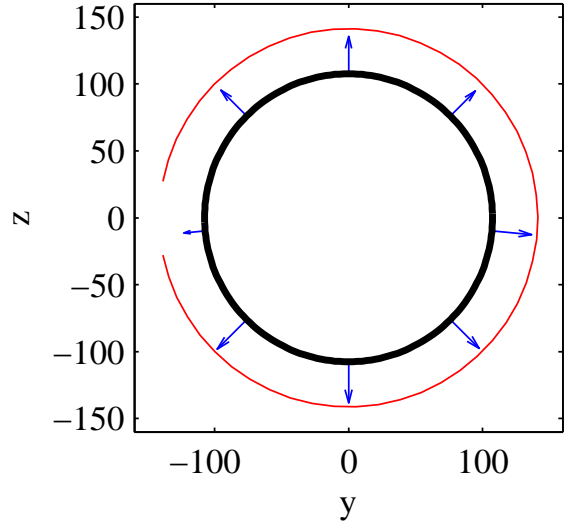

(a) $\mathrm{x}=425 \mathrm{~mm}$

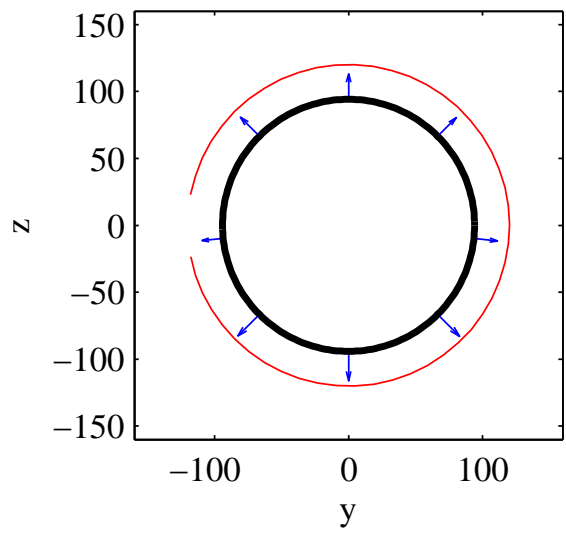

(c) $\mathrm{x}=612.5 \mathrm{~mm}$

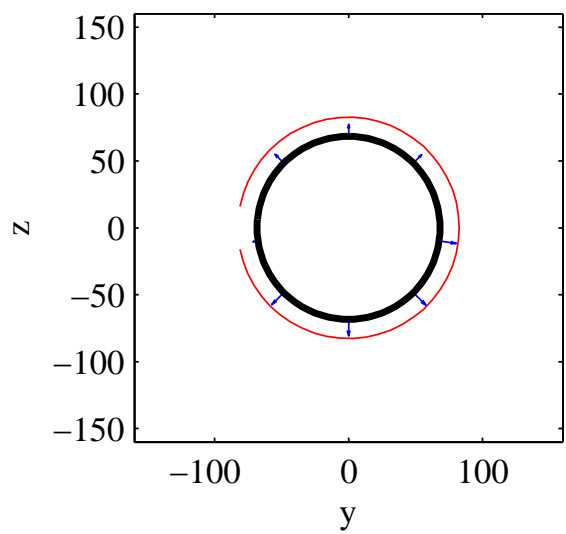

(e) $\mathrm{x}=812.5 \mathrm{~mm}$

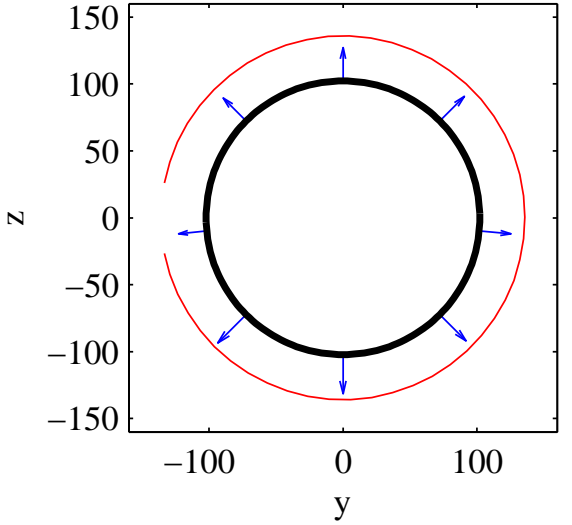

(b) $\mathrm{x}=525 \mathrm{~mm}$

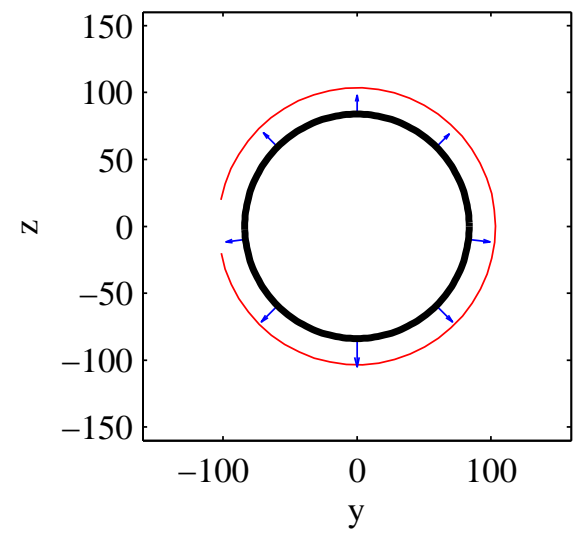

(d) $\mathrm{x}=700 \mathrm{~mm}$

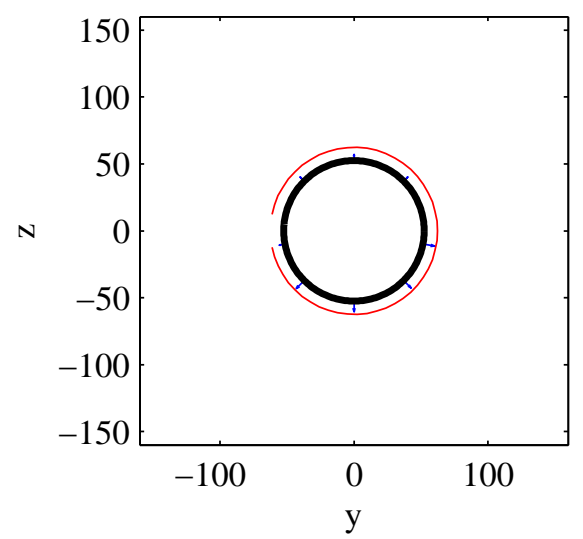

(f) $\mathrm{x}=925 \mathrm{~mm}$

Figure 10.7: Comparison of Configuration 1 experimentally measured pressure coefficients at nacelle pressure ports with computationally calculated values from the APAME panel code with the propeller on. 
deflecting toward the right wingtip and the lower portion deflecting toward the left. This behavior matches with the model of Strash and Lednicer, who also conducted validation against the AGARD Configuration 2 geometry. The final particle locations are shown from different viewpoints in Figure 10.8 and Figure 10.9 , Both figures are the result of simulation without stretching, as the more complex geometry of Configuration 2 causes the instabilities in the wake to develop even more rapidly with stretching accounted for.

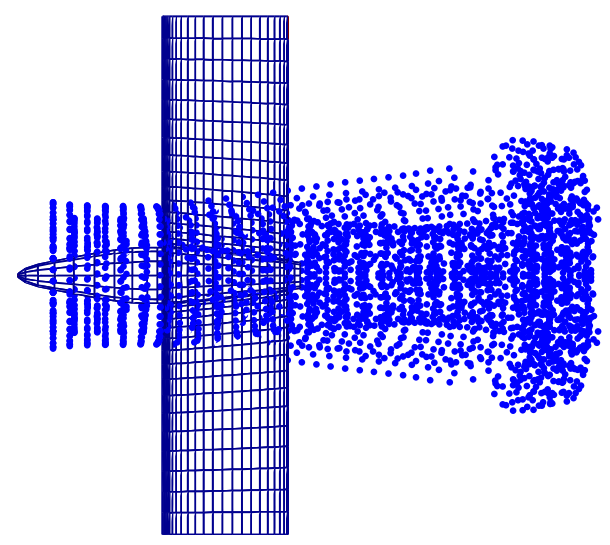

Figure 10.8: Fully convected particle locations around Configuration 2 at the end of 31 time steps.

The aerodynamic coefficients were again examined, this time for Configuration 2. The behavior overall was quite similar to what occurred in Configuration 1, with the drag coefficient fluctuating as the wake passed over the configuration, and appearing to level out towards the end of the simulation. The main difference noticeable in Configuration 2 is the presence of a roll moment coefficient, $C_{l}$. This coefficient appears because the swirl in the streamtube applies asymmetric pressure on the surface of the wing, pushing down on one half span and up on the other. The coefficients 


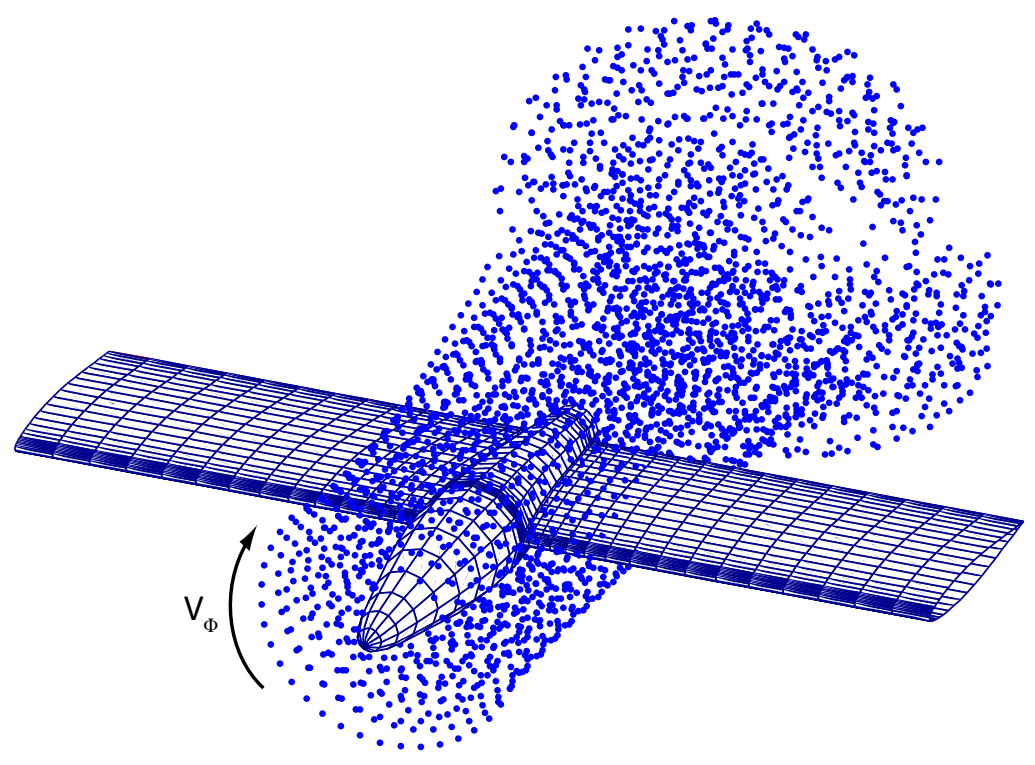

Figure 10.9: Fully convected particle locations around Configuration 2 at the end of 31 time steps, with the positive $V_{\phi}$ direction indicated, where the propeller rotation direction is the same as the positive $V_{\phi}$ direction.

are only shown for the case without stretching because of the instabilities caused by wake stretching.

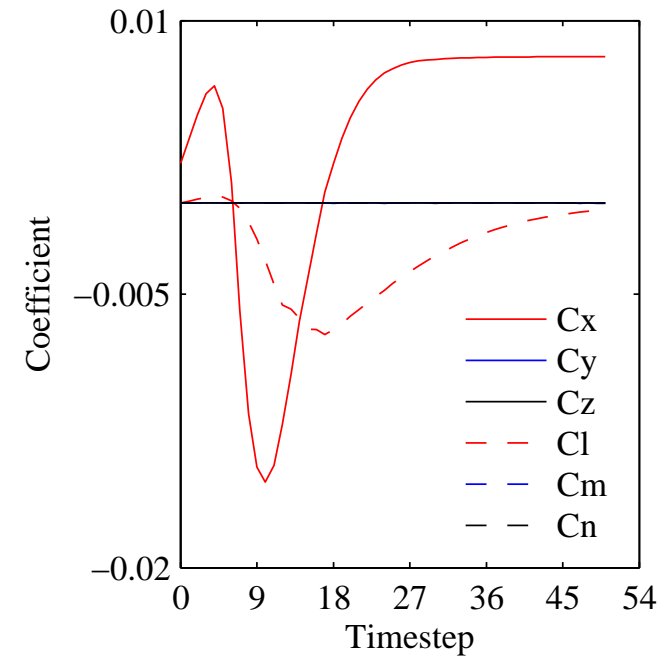

Figure 10.10: Aerodynamic coefficients for Configuration 2 without stretching turned on. 


\subsubsection{Velocity Profile Comparisons}

The velocity profiles calculated at various axial stations from the simulation are again compared with experimental data. The surveys again take place starting up on the upper surface at the centerline of the nacelle where $y=0$, and extend directly upward. The first survey occurs $10 \mathrm{~mm}$ behind the actuator disk, and is shown in Figure 10.11. As was the case with Configuration 1, the sharp gradient in the axial velocity profile at the tip of the disk is not captured in the numerical distribution. The swirl profile on the other hand, is not able to capture the sharp gradient that likely occurs near the hub of the disk, although it is difficult to tell the exact behavior of the experimental data terminating at the hub because the experimental survey did not extend that far.

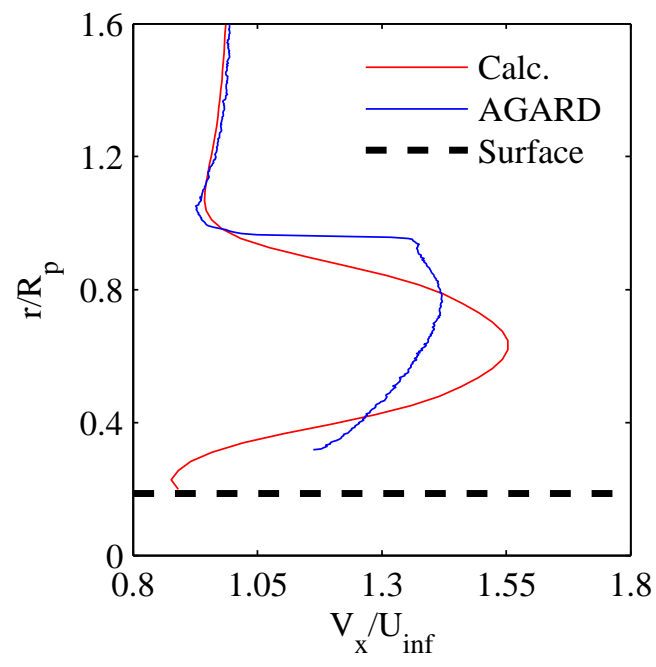

(a) Axial

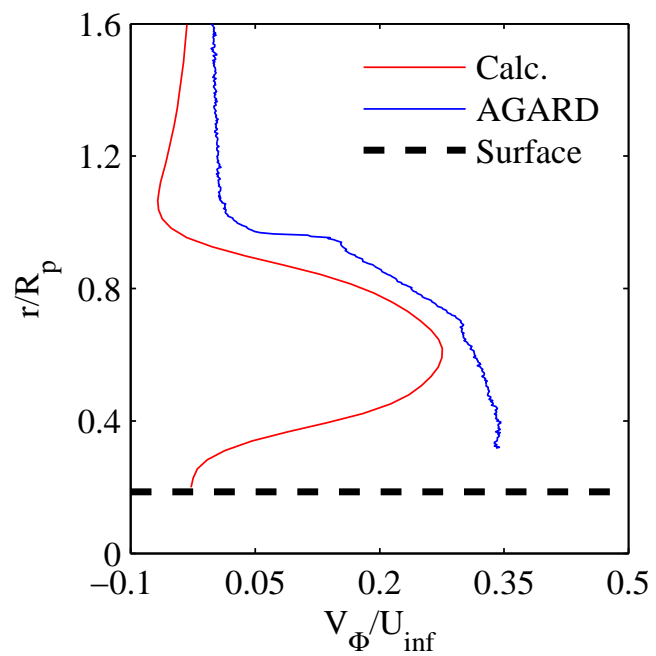

(b) Swirl

Figure 10.11: Velocity profiles at $x=10 \mathrm{~mm}$, close behind the actuator disk for Configuration 2.

As the flow is allowed to evolve further the matching between experimental and 
numerical data becomes much better, even though $x=525 \mathrm{~mm}$ is $125 \mathrm{~mm}$ past the leading edge of the wing, and correspondingly the wing-slipstream interaction has begun. The axial velocity distribution still doesn't capture the sharp discontinuity at the disk tip, but the rest of the profile matches well. The mismatch toward the tip may be the result of the axial streamtube contracting more in the numerical simulation than in the experimental flow. The swirl profile matching has greatly improved, and the experimental data is provided at this axial location within a millimeter of the surface. The velocity profiles for $x=525 \mathrm{~mm}$ are shown in Figure 10.12 .

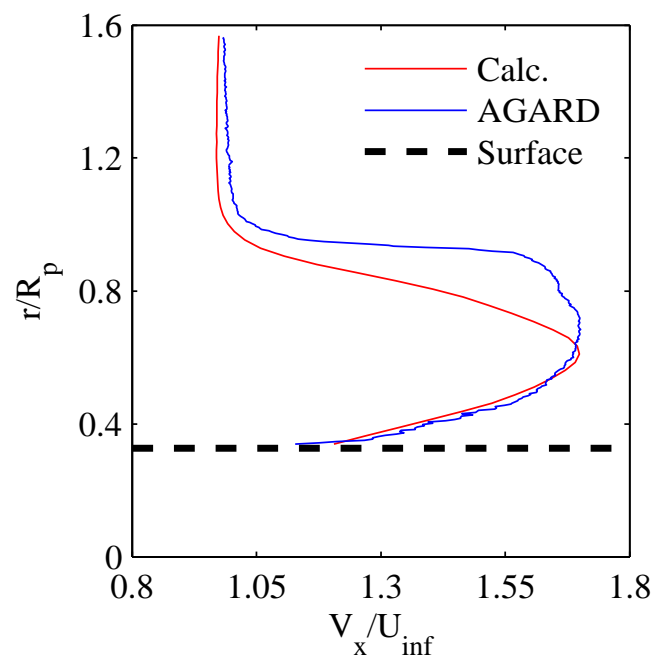

(a) Axial

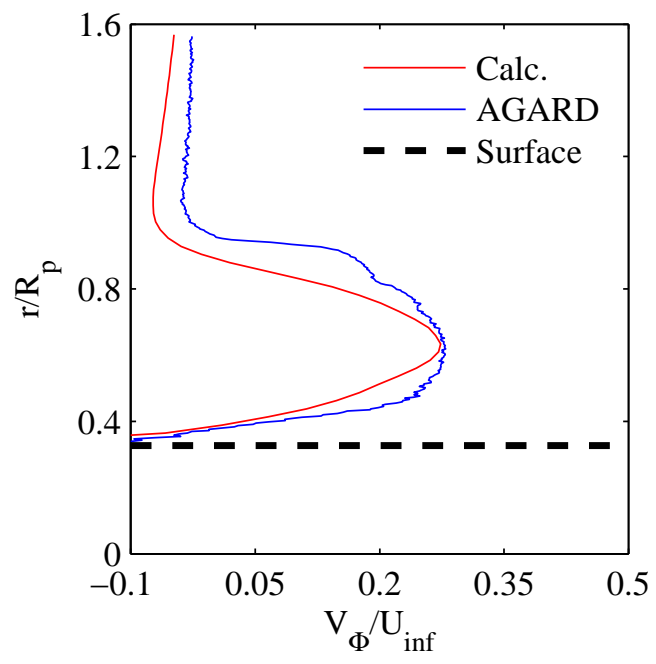

(b) Swirl

Figure 10.12: Velocity profiles at $x=525 \mathrm{~mm}$, around the middle of the nacelle for Configuration 2.

The matching at the final axial station of $x=925 \mathrm{~mm}$ is less accurate than at $x=525$. The axial velocity profile of the experiment indicates a decrease in velocity from $x=525 \mathrm{~mm}$, which is not captured at all in the numerical data. The numerical profile height decreased slightly from what it was at $x=525 \mathrm{~mm}$, providing further 
evidence that the numerical simulation was contracting at a higher rate than the experimental flow did. As with the case at $x=10$, the experimental survey was not extended all the way to the surface of the nacelle, so it is difficult to judge the matching there. The swirl profile matching is reasonable, although not as good as at $x=525 \mathrm{~mm}$. the profile shape and magnitude appear to be correct, but the numerical profile was shifted slightly closer to the nacelle than the experimental profile was. The profiles for $x=925 \mathrm{~mm}$ are shown in Figure 10.13 .

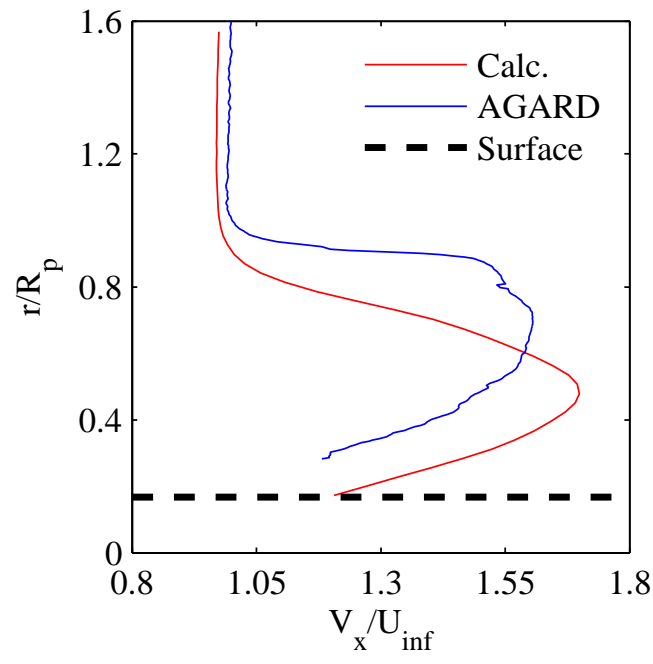

(a) Axial

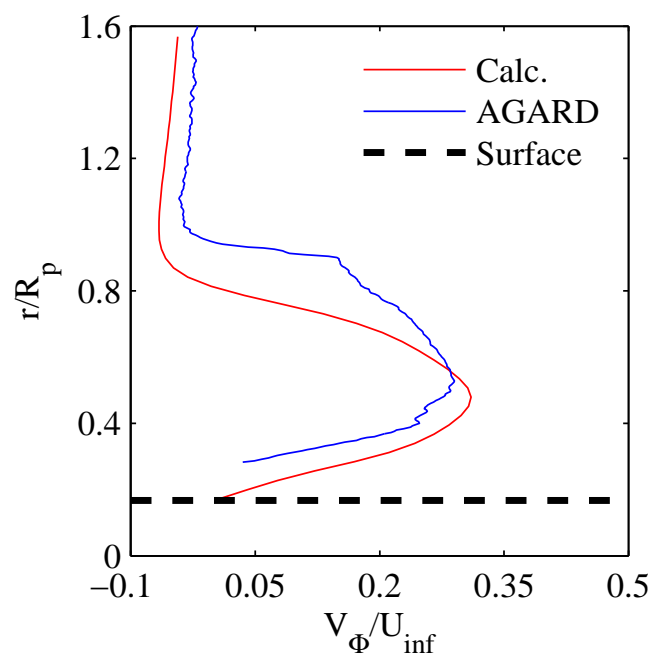

(b) Swirl

Figure 10.13: Velocity profiles at $x=925 \mathrm{~mm}$, near the end of the nacelle for Configuration 2.

\subsubsection{Nacelle Pressure Coefficients}

The pressure coefficients over the surface of Configuration 2 are now examined. Although the particle strengths are identical to those in Configuration 1, the resultant pressure coefficients are dramatically different, especially over the second half of the nacelle. This difference arises from the presence of the wing, which provides resistance 
to the swirl velocity that was not present in Configuration 1 . The resistance provided by the wing results in pressure changes along the surface of the nacelle that can be seen in Figure 10.14 and Figure 10.15. The matching is again quite good over the length of the nacelle, especially considering the relatively low particle count used to discretize the actuator disk.

\subsubsection{Wing Pressure Coefficients}

Lastly, with the wing present it was possible to examine pressure coefficient values there as well, and they are shown in Figure 10.16. The pressure coefficients are only shown for the positive half span because the simulation is symmetric, and the opposite half span would have an equal but opposite pressure distribution. The values toward the outside of the streamtube in (d) and (e) match quite well with the experimental pressure measurements. Moving inboard from there, the error between computational pressure coefficients and the experimental data becomes larger. A large portion of this discrepancy is due to the differences in the axial and swirl velocity profiles between the experiment and computation.

The axial velocity contributes to flow over the wing, which increases dynamic pressure. The swirl velocity is also diverted by the wing, so any misrepresentation of the true swirl velocity also results in misrepresented surface pressures. In addition to errors incurred by differences in velocity, the stagnation pressure change value at those points may also have error. Although the magnitudes of the calculated pressure coefficients are by no means exact, it is clear that the flow asymmetry due 


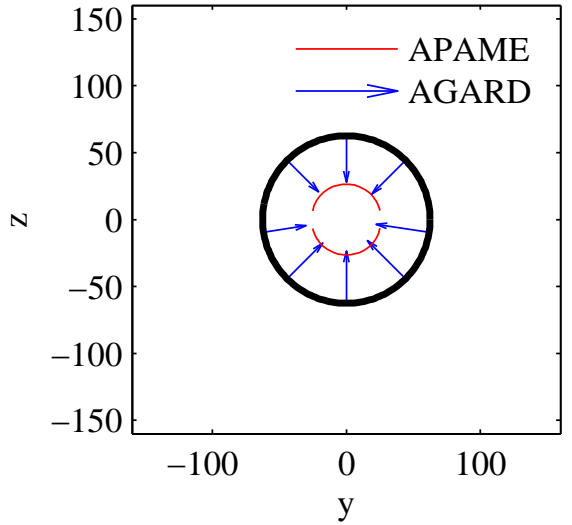

(a) $\mathrm{x}=10 \mathrm{~mm}$

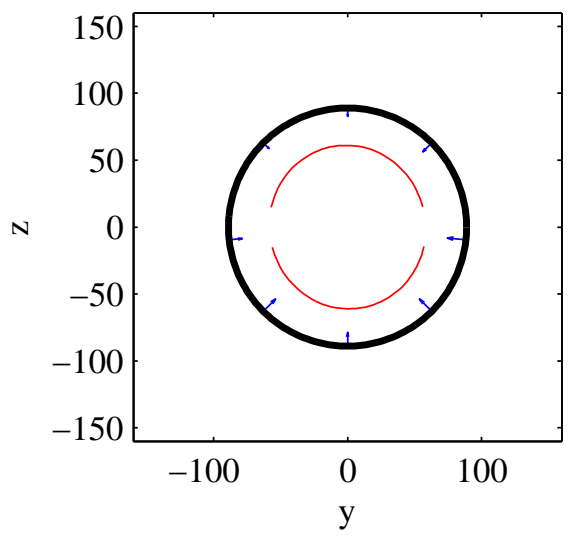

(c) $\mathrm{x}=162 \mathrm{~mm}$

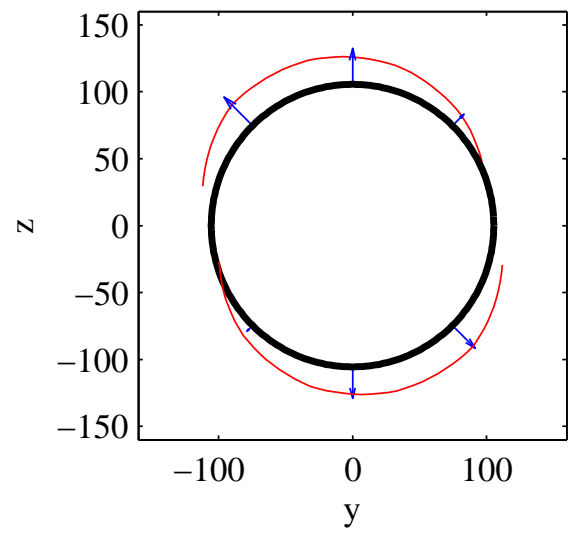

$(\mathrm{e}) \mathrm{x}=314 \mathrm{~mm}$

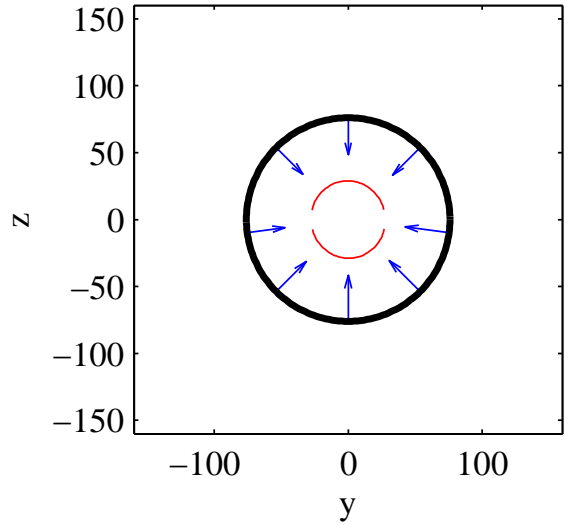

(b) $\mathrm{x}=86 \mathrm{~mm}$

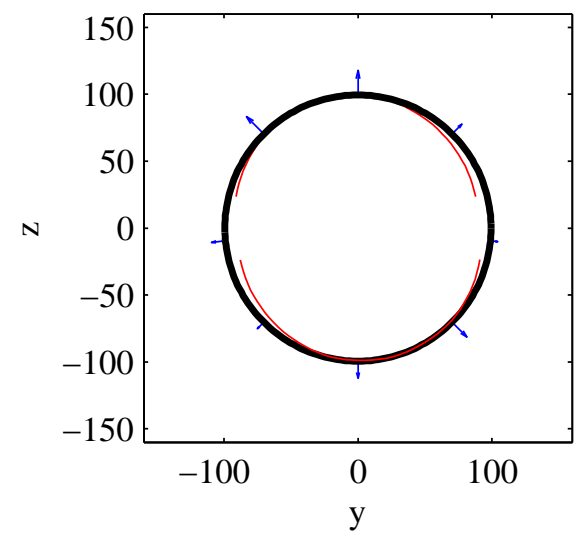

(d) $\mathrm{x}=238 \mathrm{~mm}$

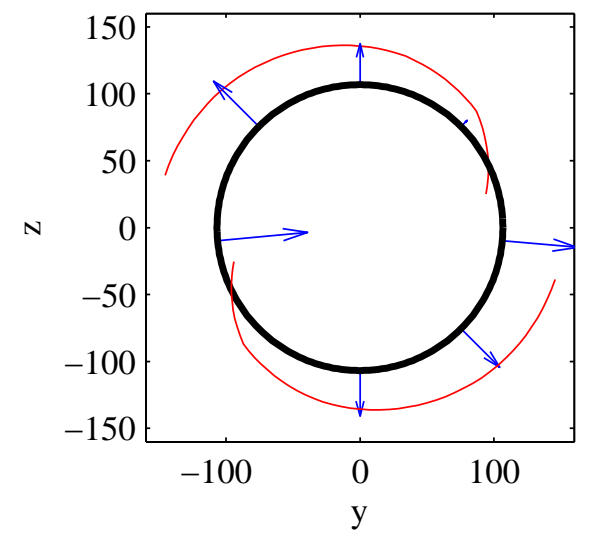

(f) $\mathrm{x}=390 \mathrm{~mm}$

Figure 10.14: Comparison of Configuration 2 experimentally measured pressure coefficients at nacelle pressure ports with computationally calculated values from the APAME panel code with the propeller on. 


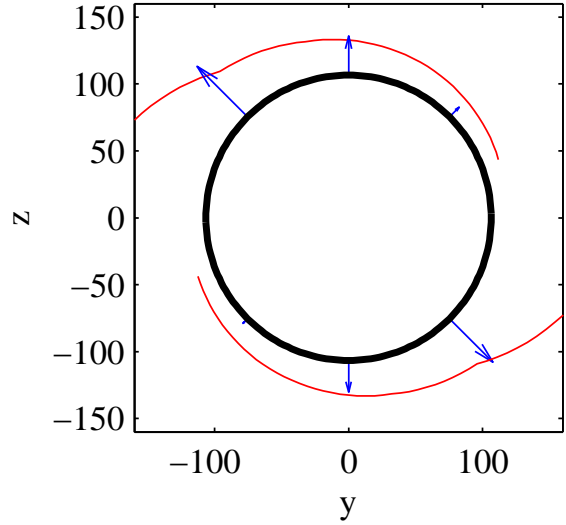

(a) $\mathrm{x}=425 \mathrm{~mm}$

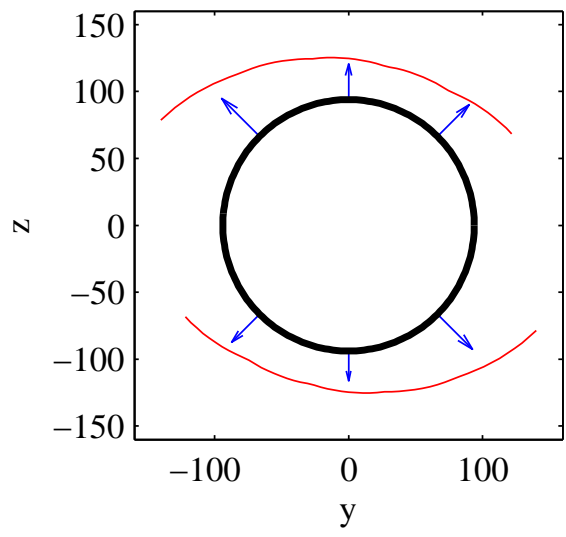

(c) $\mathrm{x}=612.5 \mathrm{~mm}$

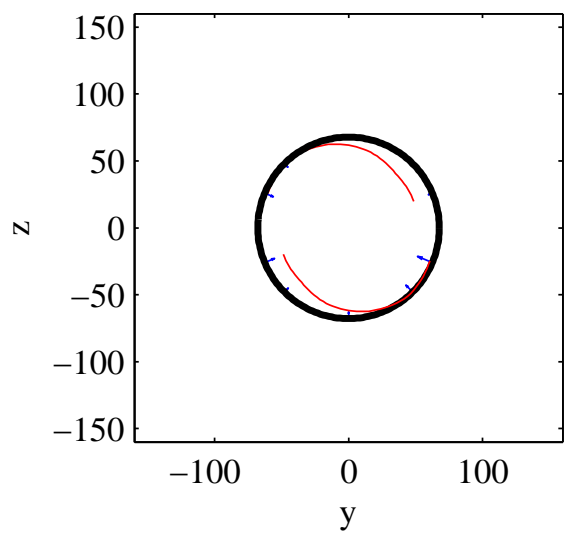

(e) $\mathrm{x}=812.5 \mathrm{~mm}$

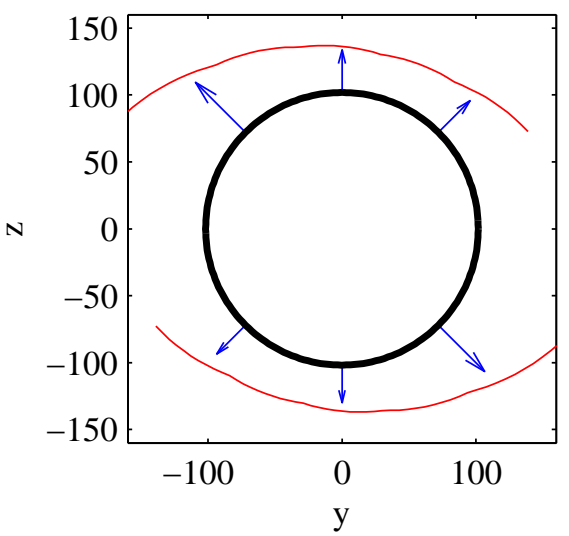

(b) $\mathrm{x}=525 \mathrm{~mm}$

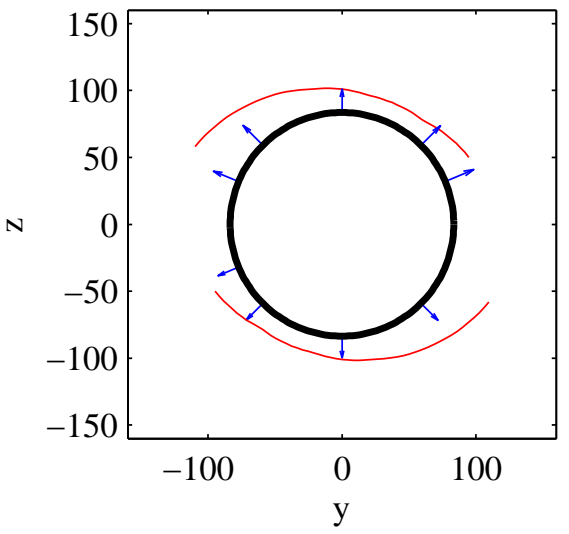

(d) $\mathrm{x}=700 \mathrm{~mm}$

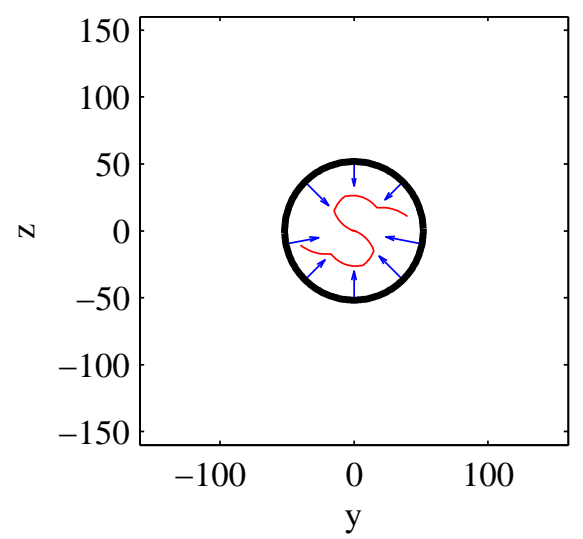

(f) $\mathrm{x}=925 \mathrm{~mm}$

Figure 10.15: Comparison of Configuration 2 experimentally measured pressure coefficients at nacelle pressure ports with computationally calculated values from the APAME panel code with the propeller on. 
to the actuator disk is being captured, because the upper and lower surface pressure distributions are no longer identical inside the streamtube.

The pressure coefficient distributions for both $y=130 \mathrm{~mm}$ and $y=195 \mathrm{~mm}$ both reflect the experimental data trends approximately. There is a great deal of disagreement at $y=260 \mathrm{~mm}$ however, and this difference stems from several possible sources. Namely, the stagnation pressure change terms applied at each of the spanwise locations was based on a very rough approximation of the correct value from the experimental data, which is only provided for $x=525 \mathrm{~mm}$. In addition to difficulty in obtaining correct stagnation pressure change terms at other axial locations on the wing, there are also differences between the values on the upper and lower surface of the wing because of the way the streamtube is sheared. The current state of the stagnation pressure change term uses the same value for both the upper and lower surface, but if a better model was implemented that could reflect the different nature of the streamtube on the upper and lower surfaces, the accuracy of the pressure coefficient distributions might be further improved.

In addition to difficulties from the stagnation pressure change, differences also likely arose from the questionable matching of the velocity profiles. Even though the velocity profiles had similar shape and magnitude to the experimental data, as shown in Figure 10.12, there was significant difference toward the tip of the disk until the streamtube was exited. This would likely explain a significant portion of why the pressures match well for values near the hub of the disk, as well outside of the 
streamtube, but have particular difficulty at $y=260 \mathrm{~mm}$.

\subsection{Channel Wing Study}

An additional case of interest that provides an example of a configuration that might expand the design space for a given trade study is that of the channel wing aircraft. The channel wing design relies on a unique wing, where a half circle shape is placed into the span, and the propeller is then mounted with its axis at the center of that circle. The propeller blades are placed at the trailing edge of the wing, and when the propeller is turned on it provides suction across the upper surface of the wing throughout the half circle. A traditional panel code would be able to solve the flow over the wing, but could not hope to account for the influence provided by the propeller, which can amount to significant lift benefits.

\subsubsection{Resultant Pseudo-Steady Flowfield}

The flowfield is solved by placing an actuator disk at the trailing edge of the wing, with the center of the disk at the center of the circle deformation in the wing. The channel wing case presented here uses particle strengths to provide similar velocity profiles to those encountered in Configuration 1 of the AGARD study. Figure 10.17 shows the convected particle locations with stretching accounted for. The simulation is on the verge of instability, which is noticeable because of the particles that have started to break out of the streamtube. The streamtube is at a distinct angle relative to the horizontal, indicating that lift is being generated, even though the airfoil is 


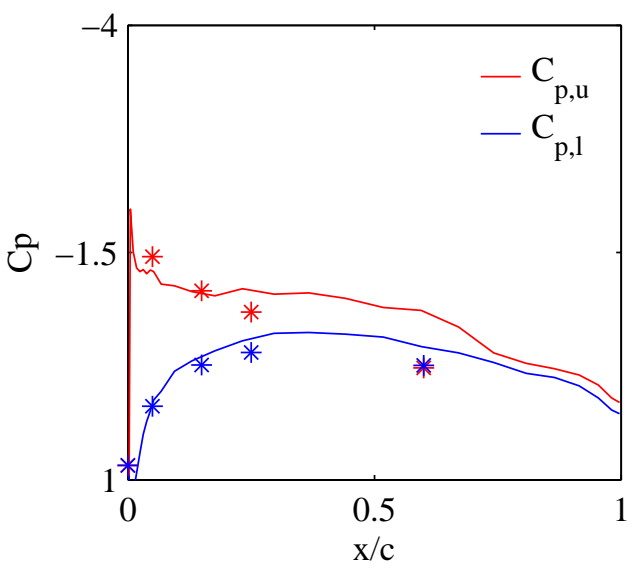

(a) $\mathrm{y}=130 \mathrm{~mm}$

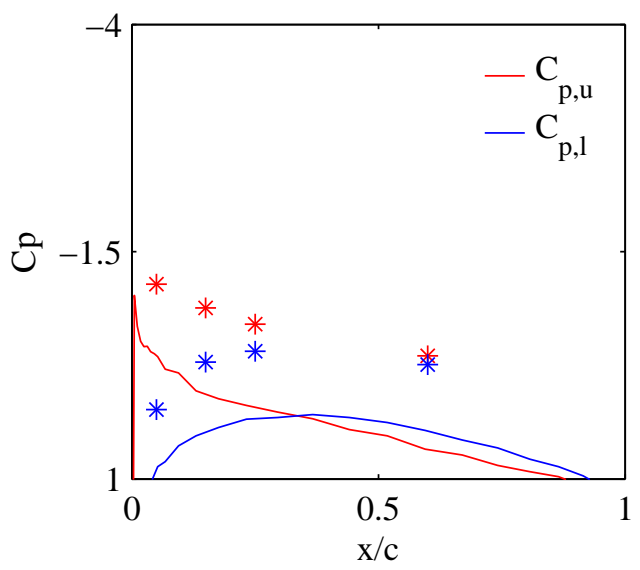

(c) $\mathrm{y}=260 \mathrm{~mm}$

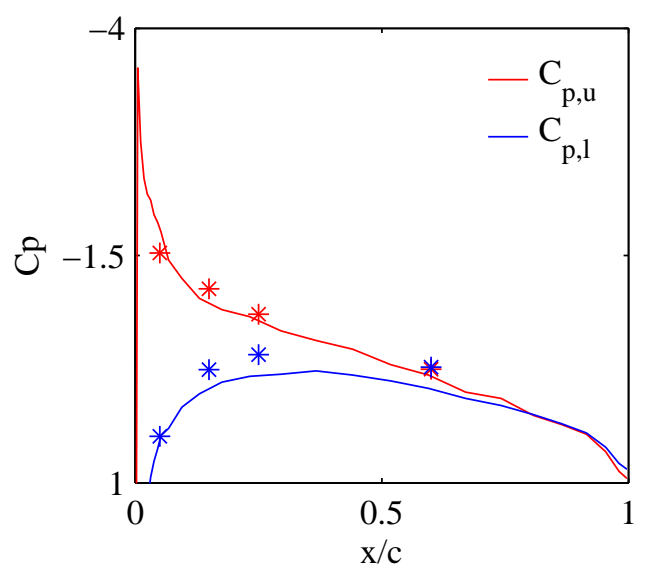

(b) $\mathrm{y}=195 \mathrm{~mm}$

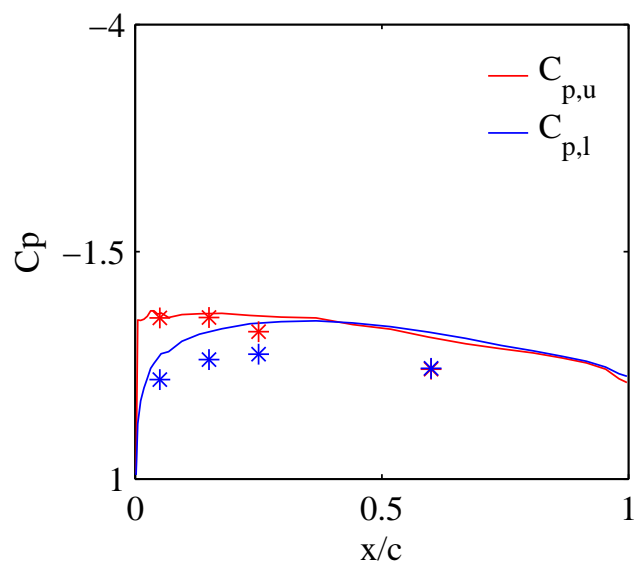

(d) $\mathrm{y}=300 \mathrm{~mm}$

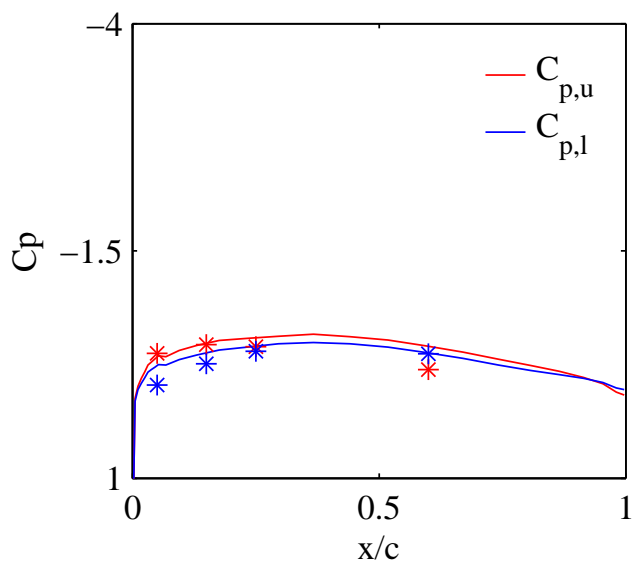

(e) $\mathrm{y}=340 \mathrm{~mm}$

Figure 10.16: Comparison of Configuration 2 experimentally measured pressure coefficients at wing pressure ports with computationally calculated values from the APAME panel code with the propeller on. 
symmetric and the wing is at zero incidence angle.

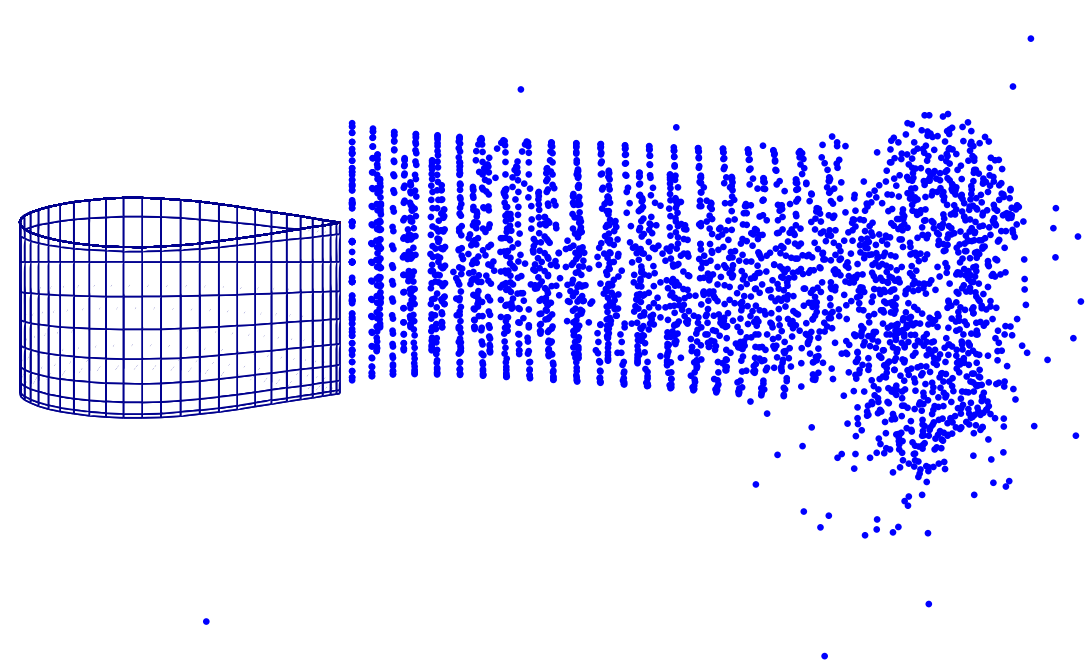

Figure 10.17: Fully convected particle locations around a channel wing at the end of 25 time steps with stretching at the onset of instability.

The coefficient history is presented for the channel wing in Figure 10.18 both with and without stretching. The comparison of the two figures shows that stretching contributes little to the coefficient evolution. The primary difference between the two solutions is that when stretching is present there is a constant change in particle strengths in the field due to the wake, which prevents the coefficients from ever leveling out. Clearly a lift coefficient $C z$ is present with the actuator disk on. The drag coefficient, $C_{x}$, is negative in both cases. This is likely the result of the pressure imbalance between the upper and lower surfaces. At the same time, induced drag is negligible because the lift generation is occurring only in the channel, while the remainder of the span has identical pressure distributions on the upper and lower surfaces. Because the panel code is inviscid, no viscous drag effects were captured, 
and the viscous drag would likely tip the $C_{x}$ value back to a reasonable number, especially considering the additional surface blowing that is caused by suction from the actuator disk.

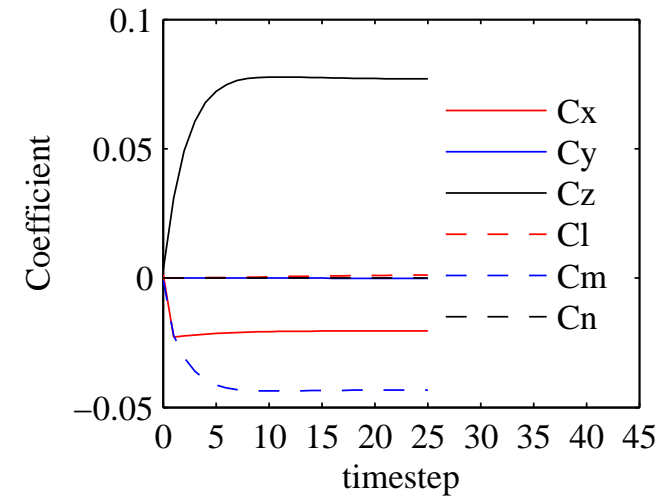

(a) No Stretching

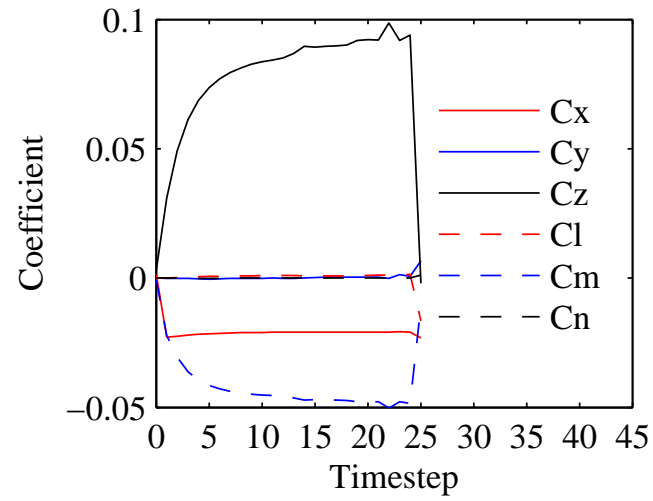

(b) With Stretching

Figure 10.18: Aerodynamic coefficients for a channel wing configuration with and without stretching turned on.

\subsubsection{Wing Pressure Coefficient Contours}

The pressure coefficient distribution over the wing can be examined before and after the effects of the actuator disk are accounted for. The changes are quite visible on the surface, particularly in the channel area where the actuator disk is increasing the flow velocity. This change in pressure distribution causes a nonzero lift coefficient over the symmetric airfoil. 


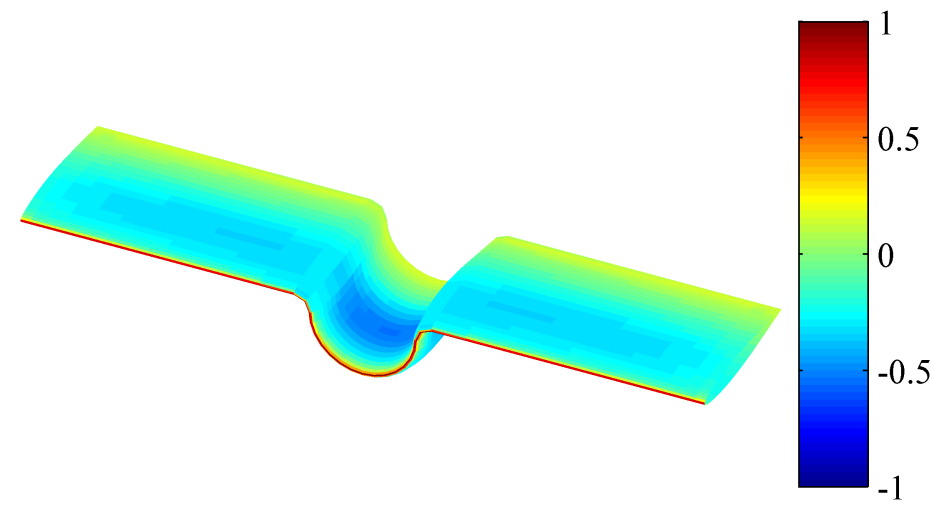

Figure 10.19: Pressure coefficient distribution around a channel wing prior to activation of the actuator disk.

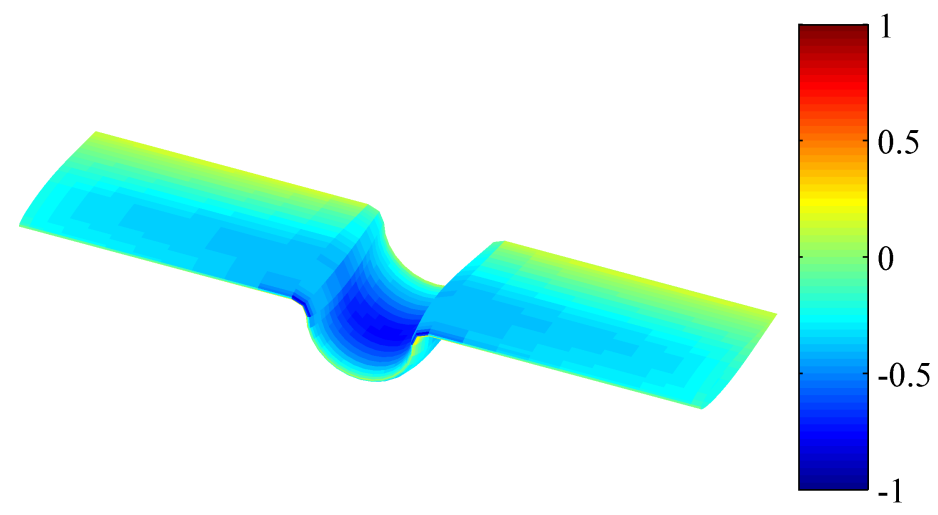

Figure 10.20: Pressure coefficient distribution around a channel wing after 25 time steps. 


\section{Chapter 11}

\section{Conclusions}

All of the components necessary to build up a combination method for propellerairframe interaction were demonstrated individually in Chapter 3 and Chapter 5 and Chapter 7. The vortex particle scheme was validated, as discussed in Chapter 4. The ability to model an actuator disk with a vortex particle scheme was demonstrated in Chapter 6. Once the individual pieces were complete, their integration was discussed in Chapter 8, and a final product was created. Next a series of experiments was introduced in Chapter 9 that provided an ideal benchmarking tool for the final form of the method. Finally, the complete model was applied to the benchmark wind tunnel cases in Chapter 10, and the results demonstrated the value of the method.

The method of using the combination of a vortex particle scheme and a panel code to capture the interference effects of a propeller has been demonstrated to be effective.

All desired qualitative trends are captured with the model, and the quantitative results are quite good for pressure coefficients on the nacelle surfaces in the AGARD validations. The results were less accurate over the wing in Configuration 2, due 
largely to the difficulty in exactly matching the velocity profiles inside the streamtube, particularly swirl.

The test cases that were run provide good evidence that the complex stretching interactions are probably not necessary to obtain approximate quantitative results. The removal of the stretching term for the particle scheme has the effect of both increasing computational efficiency due to a reduction in the necessary computations, and increasing the stability by preventing unnecessary particle strength changes in the wake. Additionally, the omission of a higher order effect like stretching may be insignificant when also considering that the panel code is inviscid, and that the strength assignment for the disk is based on simplified approximations of a true propeller.

\subsection{Limitations}

Although the combination vortex particle-panel code for modeling propeller airframe interaction appears to be a valuable new tool to the conceptual designer, there are currently some limitations to its application. The limitations provide some insight into the strengths and weaknesses of the method and help to understand when it is most applicable and when its use should be avoided in favor of other methods.

Although the particle scheme allows for modeling of rotational flow effects inside the panel code framework, the remaining limitations associated with a panel code still persist. This means that accurate panel code results can only be expected for 
incompressible and inviscid flows. Because the panel code assumes inviscid flow, and no integral boundary layer scheme is incorporated in the current MATLAB version of APAME, drag coefficient prediction cannot hope to match true flow physics.

In addition to the limitations imposed by the method itself, there are certain inherent costs associated with both the vortex particle scheme and the panel code. Specifically, the panel code requires the storage and inversion of a matrix of size $M$ by $M$, where $M$ is the number of panels on the geometry. Depending on the complexity and desired resolution of the geometry, the number of panels required may exceed the capability of a standard desktop computer. Additionally, each disk present requires particles for simulation, so more propellers present means more particles will be required to simulate the flow. A distributed propulsion system would be extremely taxing to run, and would likely be impossible on a desktop computer at the current state of the code.

\subsection{Future Work}

While a great deal has been accomplished over the course of this project, these accomplishments have provided insight into what aspects of the concept need to continue to be developed in the future. The limitations described in the prior section provide some preliminary examples of areas that require further investigation. Additionally, other needs have become apparent over the course of the work that will be addressed here. 
It became quite clear as the complexity of geometries increased throughout the course of the project that computational efficiency would be key to the success of the method in the future. The $N$-body problem already inherent in the particle scheme combined with the addition of $M$ panels whose influence must also be accounted for meant that high resolution cases could become prohibitive quickly. Throughout the course of the research it was discovered that nearly all vortex particle schemes, including those coupled with panel codes, use Fast Multipole or Fast Poisson-Boltzmann to achieve large speed increases. In addition to these efficiency inducing algorithms, the $N$-body problem is conducive to parallel computation, and the possibility of calculations being offloaded to a GPU for massive parallelizing has also been shown to reduce computational expense. One final way of increasing efficiency in certain cases is the addition of a symmetry plane capability. This capability would ideally reduce the number of individual actuator disks in a given simulation, for example by modeling only a half span rather than a complete geometry.

Initial vortex particle theory indicated that it was important to maintain vortex core overlap over the entire simulation in order to obtain the most accurate possible solution. While it is easy to ensure that particles overlap at the onset of the simulation, there is no simple way to ensure that the overlap is maintained throughout the length of the study. Maintaining overlap proves especially difficult when modeling an actuator disk, because particles near the hub are accelerated to higher velocities than those toward the tip. To solve this problem the possibility of variable vortex core 
sizes could be investigated, and a robust core sizing scheme would need to be found to provide that variation.

The wake currently used in the APAME panel code, while simple, proved to be troublesome when interacting with particles. Since the feasibility has already been demonstrated by several previous authors of using vortex particles to model the wakes shed from aircraft, a similar scheme could be implemented for wake treatment here. The added benefit exists that the infrastructure is already present to handle particle interaction, although the cost associated with adding additional particles to the field may be too prohibitive. It is likely though that with better computational efficiency through any of the techniques previously mentioned, the addition of wake particles may prove quite beneficial.

More research needs to be done into the best possible way to approximate a propeller with vortex particles, and additionally into how to relate terms useful to the conceptual designer into assigning particle strengths. While the approximations used in this research proved viable, a much more robust method of relating inputs such as thrust coefficient, power coefficient, and velocity profiles would be highly valuable. This is particularly true for swirl velocity distributions, for which no known theoretical relationship proved useful.

More research would also be valuable into ways to alleviate the influence of the unsteady wake roll up. The unsteady effects of the wake are undesirable in the solutions that this method is applicable to, and it may be possible to apply a theoretical steady 
flowfield such as those of Conway seen in Chapter 5. The steady exact actuator disk solution would then be convected downstream as particles were introduced at each time step, with the exact solution initial conditions changing based on the conditions of the final group of particles present. Another possibility is to use a technique similar to the one implemented in FASTAERO by Willis. 1 Willis states that when the particles have traveled sufficiently far away from the paneled bodies of interest, their exact position becomes less important. Because of this, once particles have reached a set distance, the influence from the particles and panels in the domain is no longer calculated at the obsolete particles. Instead they are just convected downstream with the freestream velocity. This simplification accomplishes two features, in that it reduces the $N$-body computation because of the removal of the necessity to find influence on every single particle after some time. Additionally, it eliminates the roll up in the wake after a certain amount of time, which prevents extreme stretching that might cause instability or nonphysical strengths. 


\section{Bibliography}

[1] Willis, D. J., An Unsteady, Accelerated, High Order Panel Method with Vortex Particle Wakes, Ph.D. thesis, Massachusetts Institute of Technology, 2006.

[2] Colin, P., Moreux, V., and Barillier, A., "Numerical Study of Aerodynamic High Speed Propeller Engine Integration on Transport Aircraft," 20th Congress of the International Council of the Aeronautical Sciences, International Council of the Aeronautical Sciences, 1996, pp. 2366-2380.

[3] Winckelmans, G. S., Topics in Vortex Methods for Computation of Three- and Two-Dimensional Incompressible Unsteady Flows, Doctor of philosophy, California Institute of Technology, Pasadena, California, February 1989.

[4] Morgenthal, G., Aerodynamic Analysis of Structures Using High-Resolution Vortex Particle Methods, Doctor of philosophy, University of Cambridge, October 2002.

[5] Willis, D. J., "FastAero - a precorrected FFT - Fast Multipole Tree Steady and Unsteady Potential Flow Solver," Tech. rep., Massachusetts Institute of Technology, 2005. 
[6] Opoku, D. G., Triantos, D. G., Nitzsche, F., and Voutsinas, S. G., "Rotorcraft Aerodynamic and Aeroacoustic Modelling Using Vortex Particle Methods," ICAS Congress, 2002.

[7] Lotstedt, P., "Propeller Slip-Stream Model in Subsonic Linearized Potential Flow," Journal of Aircraft, Vol. 29, No. 6, November-December 1992, pp. 10981105.

[8] E, Q., Yang, G., and Li, F., "Numerical Analysis of the Interference Effect of Propeller Slipstream on Aircraft Flowfield," Journal of Aircraft, Vol. 35, No. 1, January-February 1998, pp. 84-90.

[9] Samuelsson, I., "Experimental Investigation of Low Speed Model Propeller Slipstream Aerodynamic Characteristics Including Flow Field Surveys and Nacelle/Wing Static Pressure Measurements," Congress of the International Council of the Aeronautical Sciences, Vol. 17, FFA, The Aeronautical Research Institute of Sweden, 1990, pp. 71-84.

[10] Shapiro, A., "Film Notes for Vorticity," National Committee for Fluid Mechanics Films, 1969.

[11] Williis, D. J., Peraire, J., and White, J. K., "A Combined pFFT-multipole Tree Code, Unsteady Panel Method with Vortex Particle Wakes," International Journal for Numerical Methods in Fluids, Vol. 00, 2000, pp. 1-6. 
[12] Winckelmans, G., Cocle, R., Dufresne, L., and Capart, R., "Vortex Methods and their Application to Trailing Wake Vortex Simulation," Comptes Rendus Physique, Vol. 6, 2005, pp. 467-486.

[13] Chatelain, P., Curioni, A., Bergdorf, M., Rossinelli, D., Andreoni, W., and Koumoutsakos, P., "Billion Vortex Particle Direct Numerical Simulations of Aircraft Wakes," Computer Methods in Applied Mechanics and Engineering, Vol. 197, 2007, pp. 1296-1304.

[14] Opoku, D. G. and Nitzsche, F., "Acoustic Validation of a New Code Using Particle Wake Aerodynamics and Geometrically-Exact Beam Structural Dnyamics," 29th European Rotorcraft Forum, 2003.

[15] Conway, J. T. and Su, J., "PMAL Flow Calculations for the Aurora Aircraft Using Non-axisymmetric Propeller Actuator Disks," Canadian Aeronautics and Space Journal, Vol. 49, No. 1, March 2003, pp. 1-9.

[16] Strash, D. and Lednicer, D., "Analysis of propeller-induced aerodynamic effects," 16th AIAA Applied Aerodynamics Conference, 1998.

[17] Dang, T., "Simulations of Propeller/Airframe Interference Effects Using an Euler Corrrection Method," Journal of Aircraft, Vol. 26, 1989, pp. 994-1001. 
[18] Kuijvenhoven, J., "Validation of Propeller Slipstream Calculations Using a Multi-Block Euler Code," AIAA 8th Applied Aerodynamic Conference, Applied Aerodynamics, AIAA, AIAA, August 1990.

[19] Hess, J. and Valarezo, W., "Calculation of steady flow about propellers using a surface panel method," Journal of Propulsion and Power, Vol. 1, No. 6, January 1986, pp. 470-476.

[20] Rizk, M., "Propeller Slipstream/Wing Interaction in the Transonic Regime," Journal of Aircraft, Vol. 18, No. 3, March 1981, pp. 184-191.

[21] Whitfield, D. and Jameson, A., "Euler Equation Simulation of Propeller-Wing Interaction in Transonic Flow," Journal of Aircraft, Vol. 21, No. 11, November 1984, pp. 835-839.

[22] Samuelsson, I., "Low Speed Propeller Slipstream Aerodynamic Effects," Tech. rep., FFA, The Aeronautical Research Institute of Sweden, 1994.

[23] Samuelsson, I., "Low Speed Wind Tunnel Investigation of Propeller Slipstream Aerodynamic Effects on Different Nacelle/Wing Combinations," Congress of the International Council of the Aeronautical Sciences, Vol. 16, Congress of the International Council of the Aeronautical Sciences, August-September 1988, pp. $1749-1765$. 
[24] Fratello, G., Favier, D., and Maresca, C., "Experimental and Numerical Study of Propeller/Fixed Wing Interaction," Journal of Aircraft, Vol. 28, No. 6, June 1991, pp. 365-373.

[25] Witkowski, D. P., Lee, A. K., and Sullivan, J. P., "Aerodynamic Interaction Between Propellers and Wings," Journal of Aircraft, Vol. 26, No. 9, September 1989, pp. 829-836.

[26] Stuermer, A., "Unsteady Euler and Navier-Stokes Simulations of Propellers with Unstructured DLR TAU-Code," New Results in Numerical and experimental Fluid Mechanics V, Vol. 92, 2006, pp. 144-151.

[27] Cho, J. and Williams, M. H., "Propeller-Wing Interaction Using a Frequency Domain Panel Method," Journal of Aircraft, Vol. 27, No. 3, March 1990, pp. 196203.

[28] Rehbach, C., "Numerical Calculation of Three-Dimensional Unsteady Flows with Vortex Sheets," Aerospace Sciences Meeting, 1978.

[29] Beale, J. T. and Majda, A., "Vortex Methods I: Convergence in Three Dimensions," Mathematics of Computation, Vol. 39, No. 159, July 1982, pp. 1-27.

[30] Beale, J. T. and Majda, A., "Vortex Method II: Higher Order Accuracy in Two and Three Dimensions," Mathematics of Computation, Vol. 39, No. 159, July 1982, pp. 29-52. 
[31] Beale, J. T., "A Convergent 3-D Vortex Method With Grid-Free Stretching," Mathematics of Computation, Vol. 46, No. 174, April 1986, pp. 401-424.

[32] Saffman, P. and Meiron, D., "Difficulties with Three-Dimensional Weak Solutions for Inviscid Incompressible Flow," Phys. Fluids, Vol. 29, No. 8, August 1986, pp. 2373-2375.

[33] Cottet, G.-H., "A new approach for the analysis of Vortex Methods in two and three dimensions," Ann. Inst. Henri Poincare, Vol. 5, No. 3, 1988, pp. 227-285.

[34] Winckelmans, G. and Leonard, A., "Contributions to Vortex Particle Methods for the Computation of Three-Dimensional Incompressible Unsteady Flows," Journal of Computational Physics, Vol. 109, 1993, pp. 247-273.

[35] Degond, P. and Mas-Gallic, S., "The Weighted Particle Method for ConvectionDiffusion Equations Part 1:The Case of an Isotropic Viscosity," Mathematics of Computation, Vol. 53, No. 188, October 1989, pp. 485-507.

[36] Degond, P. and Mas-Gallic, S., "The Weighted Particle Method for ConvectionDiffusion Equations Part 2: The Anisotropic Case," Mathematics of Computation, Vol. 53, No. 188, October 1989, pp. 509-525.

[37] Choquin, J. and Huberson, S., "Particle Simulation of Viscous Flow," Computers and Fluids, Vol. 17, No. 2, 1989, pp. 397-410. 
[38] Benfatto, G., Picco, P., and Pulvirenti, M., "On the Invariant Measures for the Two-Dimensional Euler Flow," Journal of Statistical Physics, Vol. 46, No. 3/4, 1987, pp. 729-742.

[39] Cottet, G.-H. and Koumoutsakos, P. D., Vortex Methods: Theory and Practice, Cambridge University Press, 2000.

[40] Beale, J., "On The Accuracy of Vortex Methods at Large Times," Tech. rep., Institute for Mathematics and its Applications, 1987.

[41] Williamson, J., "Low-Storage Runge-Kutta Schemes," Journal of Computational Physics, Vol. 35, 1980, pp. 48-56.

[42] Carpenter, M. H. and Kennedy, C. A., "Fourth-Order 2N-Storage Runge-Kutta Schemes," NASA Technical Memorandum 109112, NASA, June 1994.

[43] Carpenter, M. H. and Kennedy, C. A., "Third-Order 2N-Storage Runge-Kutta Schemes with Error Control," NASA Technical Memorandum 109111, NASA, June 1994.

[44] Wilson, R. V., Demuren, A. O., and Carpenter, M., "Higher-Order Compact Schemes for Numerical Simulation of Incompressible Flows," NASA ICASE Report 98-13, NASA, February 1998. 
[45] Kennedy, C. A., Carpenter, M. H., and Lewis, R. M., "Low-Storage, Explicit Runge-Kutta Schemes for the Compressible Navier-Stokes Equations," NASA CR ICASE 99-22, NASA, 1999.

[46] Calvo, M., Franco, J., and Randez, L., "A New Minimum Storage RungeKutta Scheme for Computational Acoustics," Journal of Computational Physics, Vol. 201, 2004, pp. 1-12.

[47] Butcher, J. C., The Numerical Analysis of Ordinary Differential Equations: Runge-Kutta and General Linear Methods, Wiley Interscience, 1987.

[48] Conway, J. T., "Analytical Solutions for the Actuator Disk with Variable Radial Distribution of Load," Journal of Fluid Mechanics, Vol. 297, 1995, pp. 327-355.

[49] Conway, J. T., "Exact Actuator Disk Solutions For Non-uniform Heavy Loading and Slipstream Contraction," Journal of Fluid Mechanics, Vol. 365, 1998, pp. $235-267$.

[50] Conway, J. T., "Prediction of the Performance of Heavily Loaded Propellers with Slipstream Contraction," Canadian Aeronautics and Space Journal, Vol. 44, No. 3, September 1998, pp. 169-174.

[51] Hough, G. and Ordway, D., "The Generalized Actuator Disk," Developments in Theoertical and Applied Mechanics, Vol. 2, 1965, pp. 317-336.

[52] Conway, J., "Personal Correspondence with Rob McDonald," 9/4/2009. 
[53] VonMises, R., Theory of Flight, McGraw-Hill, 1945.

[54] Lamb, S. H., Hydrodynamics, Dover Publications, 1945.

[55] Strain, J., "2D Vortex Methods and Singular Quadrature Rules," Journal of Computational Physics, Vol. 124, No. 49, 1996, pp. 131-145.

[56] Leonard, A., Koumoutsakos, P., and Winckelmans, G., "Vortex Methods for Three-Dimensional Separated Flows," Fourteenth International Conference on Numerical Methods in Fluid Dynamics, Vol. 453, 1995, pp. 21-30.

[57] Winckelmans, G., Salmon, J., Warren, M., and Leonard, A., "Application of Fast Parallel and Sequential Tree Codes to Computing Three-Dimensional Flows with Vortex Element and Boundary Element Methods," ESAIM, Vol. 1, 1996, pp. 225-240.

[58] Voutsinas, S., "Vorrtex Methods In Aeronautics: How to Make Things Work," International Journal of Computational Fluid Dynamics, Vol. 20, No. 1, January 2006, pp. 3-18.

[59] Stock, M. J. and Gharakhani, A., "Toward Efficient GPU-accelerated N-body Simulations," Tech. rep., 46th AIAA Aerospace Sciences Meeting, January 2008.

[60] Stone, C., Duque, E., Hennes, C., and Gharakhani, A., "Rotor Wake Modeling with a Coupled Eulerian and Vortex Particle Method," Aerospace Sciences Meeting Including the New Horizons Forum and Aerospace Exhibition, 2010. 
[61] Hess, J. L. and Smith, A., "Calculation of Non-Lifting Potential Flow About Arbitrary Three-Dimensional Bodies," Tech. rep., Douglas Aircraft Company, 1962.

[62] Choi, S., Alonso, J. J., and Kroo, I. M., "Two-Level Multi-Fidelity Design Optimization Studies for Supersonic Jets," AIAA, 2005.

[63] Walsh, J., Townsend, J., Salas, A., Samareh, A., Mukhopadhyay, V., and Barthelemy, J., "Multidisciplinary High-Fidelity Analysis and Optimization of Aerospace Vehicles, Part 1: Formulation," AIAA, 2000.

[64] Alonso, J. and Kroo, I., "Advanced Algorithms for Design and Optimization of Quiet Supersonic Platforms," AIAA Aerospace Sciences Meeting and Exhibit, 2002.

[65] Filkovic, D., APAME - Aircraft Panel Method Documentation/Tutorial, July 2009.

[66] Katz, J. and Plotkin, A., Low-Speed Aerodynamics, Cambridge University Press, 2001.

[67] Browne, L. E. and Ashby, D. L., "Study of the Integration of Wind Tunnel and Computational Methods for Aerodynamic Configurations," NASA Technical Memorandum 102196, NASA, October 1989. 
[68] Maskew, B., Program VSAERO Theory Document, NASA, contract report 4023 ed., September 1987.

[69] Takahashi, T., "On the decomposition of drag components from wake flow measurements," AIAA Aerospace Sciences Meeting EG Exhibit, 1997. 


\section{Appendix A}

\section{Particle Cell Area Derivation}

The area for a single cell in the discretized disk of particles shown in Figure 4.1 follows the equation for the area of a circle. At first glance this is counterintuitive, since clearly the shape of the cells are not circular. It can be shown however, that the area equation for a cell is in fact an exact relationship for the area of that cell.

To understand the origin of the cell area equation, first examine the area of a given layer, $n$. The area of that layer is the area of the circle defined by the lower layer radius subtracted from the area of the circle defined by the upper layer radius,

$$
A_{\text {layer }}=\pi r_{2}^{2}-\pi r_{1}^{2}=\pi\left(r_{2}^{2}-r_{1}^{2}\right)
$$

Any given layer $n$ is divided into a number of equal area cells distributed circumferentially around it. For layer $n$ there are $8 n$ cells. Thus, the area for a single cell is

$$
A_{\text {cell }}=\frac{A_{\text {layer }}}{8 n}
$$

This relationship applies for each cell in any layer, which can be demonstrated by several examples. For $n=1, r_{1}=r_{l}, r_{2}=3 r_{l}$, and $8 n=8$. The area of layer one is 
then

$$
A_{\text {layer }}=\pi\left(\left(3 r_{l}\right)^{2}-\left(r_{l}\right)^{2}\right)=\pi\left(9 r_{l}^{2}-r_{l}^{2}\right)=\pi\left(8 r_{l}^{2}\right)
$$

Applying this layer area to Equation A.2 yields

$$
A_{\text {cell }}=\frac{\pi 8 r_{l}^{2}}{8}=\pi r_{l}^{2}
$$

To prove that this is in fact a general equation, and not simply a special circumstance, we can examine another $n$ value, this time $n=3$. When $n=3 r_{1}=5 r_{l}$, $r_{2}=7 r_{l}$, and $8 n=24$. The area of layer $n=3$ becomes

$$
A_{\text {layer }}=\pi\left(\left(7 r_{l}\right)^{2}-\left(5 r_{l}\right)^{2}\right)=\pi\left(49 r_{l}^{2}-25 r_{l}^{2}\right)=\pi\left(24 r_{l}^{2}\right) .
$$

The area of a cell in layer $n=3$ then becomes

$$
A_{\text {cell }}=\frac{\pi 24 r_{l}^{2}}{24}=\pi r_{l}^{2}
$$




\section{Appendix B}

\section{Thrust Coefficient Derivation}

The thrust coefficient of an actuator disk in some arbitrary flowfield can be determined through the relationship thrust shares with perturbation velocity influence. According to Conway,

$$
d T=2 \pi \rho r\left(V_{z}(r, \infty)\left[U_{\infty}+V_{z}(r, \infty)\right]-\frac{V_{\phi}(r, \infty)}{2}\right) d r,
$$

thus the differential thrust of some differential radial portion of the actuator disk is related to the axial and azimuthal perturbation velocity influences at that radial location. The relationship in Equation B.1 stems from the pressure discontinuity at the disk, which is written as

$$
\Delta P(r)=\rho\left(V_{z}(r, \infty)\left(U_{\infty}+V_{z}(r, \infty)\right)-\frac{\rho V_{\phi}(r, \infty)^{2}}{2} .\right.
$$

The thrust over a differential annular ring of the disk is

$$
d T=P d A
$$

where $d A=2 \pi r d r$. Equation B.1 can be integrated over the disk, resulting in

$$
\int d T=\int_{R_{h}}^{R_{a}} 2 \pi \rho r\left(V_{z}(r, \infty)\left[U_{\infty}+V_{z}(r, \infty)\right]-\frac{V_{\phi}(r, \infty)}{2}\right) d r .
$$


Since the integral of a sum is the sum of the integrals, Equation B.4 becomes

$$
T=2 \pi \rho\left[\int_{R_{h}}^{R_{a}} r V_{z}(r, \infty) U_{\infty} d r+\int_{R_{h}}^{R_{a}} r V_{z}(r, \infty)^{2}-\int_{R_{h}}^{R_{a}} r \frac{V_{\phi}(r, \infty)}{2} d r\right] .
$$

By definition, the thrust coefficient, $C_{T}$, is

$$
C_{T} \equiv \frac{T}{\frac{1}{2} \rho U_{\infty}^{2} S}
$$

Solving Equation B.6 for $T$, and substituting it into Equation B.5 cancels the density terms and yields

$$
\frac{1}{2} U_{\infty}^{2} S C_{T}=2 \pi\left[\int_{R_{h}}^{R_{a}} r V_{z}(r, \infty) U_{\infty} d r+\int_{R_{h}}^{R_{a}} r V_{z}(r, \infty)^{2}-\int_{R_{h}}^{R_{a}} r \frac{V_{\phi}(r, \infty)}{2} d r\right] .
$$

Solving for $C_{T}$ results in

$$
C_{T}=\frac{4 \pi}{U_{\infty}^{2} S}\left[\int_{R_{h}}^{R_{a}} r V_{z}(r, \infty) U_{\infty} d r+\int_{R_{h}}^{R_{a}} r V_{z}(r, \infty)^{2}-\int_{R_{h}}^{R_{a}} r \frac{V_{\phi}(r, \infty)}{2} d r\right]
$$

The area $S$ defining the actuator disk is simply the area defined by a circle of radius $R_{a}$. If the hub radius, $R_{h}$, is nonzero then the smaller area circle defined by radius $R_{h}$ is subtracted. Thus

$$
S=\pi R_{a}^{2}-\pi R_{h}^{2}=\pi\left(R_{a}^{2}-R_{h}^{2}\right)
$$

Substituting this definition in results in the most general form of the $C_{T}$ integral,

$$
C_{T}=\frac{4}{U_{\infty}^{2}\left(R_{a}^{2}-R_{h}^{2}\right)}\left[\int_{R_{h}}^{R_{a}} r V_{z}(r, \infty) U_{\infty} d r+\int_{R_{h}}^{R_{a}} r V_{z}(r, \infty)^{2}-\int_{R_{h}}^{R_{a}} r \frac{V_{\phi}(r, \infty)}{2} d r\right]
$$


If it is valid to assume a lightly loaded propeller, in which case $V_{z}(r, \infty)=2 V_{z}(r, 0)$, then $C_{T}$ can be redefined as

$$
C_{T}=\frac{4}{U_{\infty}^{2}\left(R_{a}^{2}-R_{h}^{2}\right)}\left[2 \int_{R_{h}}^{R_{a}} r V_{z}(r, 0) U_{\infty} d r+\int_{R_{h}}^{R_{a}} r V_{z}(r, 0)^{2}-\int_{R_{h}}^{R_{a}} r \frac{V_{\phi}(r, \infty)}{2} d r\right] .
$$

While seemingly insignificant, this simplification is vital to many solutions with this method, because the perturbation velocity at the disk, $V_{z}(r, 0)$ can be directly related to vortex strength, and therefore particle strength, while the downstream perturbation is much less useful.

Finally, for a contra-rotating case the swirl velocity, $V_{\phi}(r, \infty)$, is zero, so Equation B.11 reduces to

$$
C_{T}=\frac{4}{U_{\infty}^{2}\left(R_{a}^{2}-R_{h}^{2}\right)}\left[2 \int_{R_{h}}^{R_{a}} r V_{z}(r, 0) U_{\infty} d r+\int_{R_{h}}^{R_{a}} r V_{z}(r, 0)^{2}\right] .
$$

\section{B.1 Iterative Coefficient Solution}

The overall goal of work with the thrust coefficient was to be able to input a thrust coefficient and assign particle strengths based on the desired thrust coefficient. To that end, once a viable method for calculating thrust coefficient was established, a routine was constructed to scale velocity distributions. This would allow the user to specify a velocity distribution shape from hub to tip of the propeller, along with a thrust coefficient, and the code would assign particle strengths from that information.

To solve for information necessary to assign particle strengths, the function for solving thrust coefficient, modeled after Equation B.12, is placed inside a MATLAB 
one dimensional gradient based optimizer called fminbnd, where the function being minimized was to be found was

$$
f=\left|C_{T, \text { desired }}-C_{T, \text { calculated }}\right| .
$$

Thus the optimizer adjusted the scale of the user selected velocity distribution by a factor, where the final factor resulted in the thrust coefficient calculated using the scaled velocity distribution being equal to the input desired thrust coefficient. 


\section{Appendix C}

\section{Derivation of Panel Stretching Influence}

To determine the stretching influence caused by each panel on all of the particles, it is necessary to understand the fundamental stretching equation that stems from the three-dimensional momentum equation. The momentum equation for a constant density fluid can be written as

$$
\frac{\partial \mathbf{u}}{\partial t}+\boldsymbol{\omega} \times \mathbf{u}=-\nabla\left(\frac{p}{\rho}+\frac{\mathbf{u} \cdot \mathbf{u}}{2}\right)+\nu \nabla^{2} \mathbf{u} .
$$

The three-dimensional vorticity equation is found by taking the curl of Equation C.1. which gives

$$
\frac{\partial \boldsymbol{\omega}}{\partial t}+\nabla \times(\boldsymbol{\omega} \times \mathbf{u})=\nu \nabla^{2} \boldsymbol{\omega} .
$$

Applying the fact that $\nabla \cdot \mathbf{u}=0$ and $\nabla \cdot \boldsymbol{\omega}=\nabla \cdot(\nabla \times \mathbf{u})=0$ to Equation C.2, it can be rewritten as

$$
\frac{\partial \boldsymbol{\omega}}{\partial t}+(\mathbf{u} \cdot \nabla) \boldsymbol{\omega}=(\boldsymbol{\omega} \cdot \nabla) \mathbf{u}+\nu \nabla^{2} \boldsymbol{\omega}
$$




\section{C.1 Transpose Scheme}

The vector particle strength evolution equation for the transpose scheme without viscous effects can be written in computational form as

$$
\frac{d}{d t} \boldsymbol{\alpha}^{p}(t)=\left(\boldsymbol{\alpha}^{p}(t) \cdot \nabla^{T}\right) \mathbf{u}^{p}\left(\mathbf{x}^{p}(t), t\right)
$$

Thus, the strength vector for a particle, $p$, is a function of the strength of that particle, $\boldsymbol{\alpha}^{p}$, the gradient operator, and the velocity of that particle, $\mathbf{u}^{p}$. Rewriting Equation C.4 using suffix notation gives

$$
\frac{d}{d t} \alpha_{i}^{p}=\alpha_{l}^{p} \frac{\partial u_{l}}{\partial x_{i}}
$$

which tells us that the magnitude of stretching that occurs depends on the velocity gradient at the location of particle $p$. In the combination method proposed here, where a panel code is also present, the gradient of the velocity at a particle location is composed of the sum of the gradient of the velocity influence due to other particles, and the gradient of velocity due to any panels present. Because the derivative of a sum is the sum of a derivative, Equation C.5 to be rewritten as

$$
\frac{d}{d t} \alpha_{i}^{p}=\alpha_{l}^{p}\left(\frac{\partial u_{l, p a r t i c l e}}{\partial x_{i}}+\frac{\partial u_{l, p a n e l}}{\partial x_{i}}\right),
$$

where $\alpha_{l}^{p}$ can be distributed to obtain

$$
\frac{d}{d t} \alpha_{i}^{p}=\left(\alpha_{l}^{p} \frac{\partial u_{l, p a r t i c l e}}{\partial x_{i}}\right)+\left(\alpha_{l}^{p} \frac{\partial u_{l, \text { panel }}}{\partial x_{i}}\right)
$$

Equation C.7 shows that the stretching influence from the particles, and that from the panels can be calculated independently and then summed. This allows the particle 
stretching influence to be calculated using existing particle relationships, while the panel stretching influence can be found and added in separately.

Examining the stretching influence from the panels in more detail

$$
\frac{d}{d t} \alpha_{i, \text { panel }}^{p}=\alpha_{l}^{p} \frac{\partial u_{l, p a n e l}}{\partial x_{i}}
$$

leads to equations for the stretching term in each vector axis

$$
\begin{aligned}
& \frac{d}{d t} \alpha_{x, \text { panel }}^{p}=\alpha_{x}^{p} \frac{\partial u_{\text {panel }}}{\partial x}+\alpha_{y}^{p} \frac{\partial v_{\text {panel }}}{\partial x}+\alpha_{z}^{p} \frac{\partial w_{\text {panel }}}{\partial x} \\
& \frac{d}{d t} \alpha_{y, \text { panel }}^{p}=\alpha_{x}^{p} \frac{\partial u_{\text {panel }}}{\partial y}+\alpha_{y}^{p} \frac{\partial v_{\text {panel }}}{\partial y}+\alpha_{z}^{p} \frac{\partial w_{\text {panel }}}{\partial y} \\
& \frac{d}{d t} \alpha_{z, \text { panel }}^{p}=\alpha_{x}^{p} \frac{\partial u_{\text {panel }}}{\partial z}+\alpha_{y}^{p} \frac{\partial v_{\text {panel }}}{\partial z}+\alpha_{z}^{p} \frac{\partial w_{\text {panel }}}{\partial z}
\end{aligned}
$$

This results in nine partial derivatives of panel velocity influences that must be taken, three with respect to each vector axis. Additionally, due to the construction of the panel code selected for this research, each panel has both a constant strength source influence and doublet influence, and for computational efficiency these influences are reduced to point elements for distances over five panel lengths away. This means that each of the nine partial derivatives will really have four terms, two doublet and two source, where only one of each type will be active depending on the distance of the survey point from the panel. The following subsections will provide the derivations for the stretching influence from each panel element type. 


\section{C.1.1 Point Source}

The velocity influence equations for a point source are

$$
\begin{aligned}
& u_{p t, \text { source }}=\frac{\sigma A\left(x-x_{0}\right)}{4 \pi\left[\left(x-x_{0}\right)^{2}+\left(y-y_{0}\right)^{2}+(z)^{2}\right]^{3 / 2}}, \\
& v_{p t, \text { source }}=\frac{\sigma A\left(y-y_{0}\right)}{4 \pi\left[\left(x-x_{0}\right)^{2}+\left(y-y_{0}\right)^{2}+(z)^{2}\right]^{3 / 2}}, \\
& w_{p t, \text { source }}=\frac{\sigma A(z)}{4 \pi\left[\left(x-x_{0}\right)^{2}+\left(y-y_{0}\right)^{2}+(z)^{2}\right]^{3 / 2}},
\end{aligned}
$$

where $\sigma$ is the point source strength, $A$ is the panel area, and $\left(x_{0}, y_{0}, z_{0}\right)$ is the element location in panel coordinates, while $(x, y, z)$ is the survey point location in panel coordinates. To obtain the stretching influence contributed by a point source element, the derivative of Equations C.12, C.13, and C.14 must be taken with respect to each axis of the survey point. Since in this case the survey point is actually a particle location, $(x, y, z)$ will now be $\left(x^{p}, y^{p}, z^{p}\right)$, and since the point element location is at the panel collocation point $\left(x_{0}, y_{0}, z_{0}\right)$ will now be $(c x, c y, c z)$.

First, examining the derivative of Equation C.12 with respect to $x^{p}$, we use the product rule

$$
\frac{d(u v)}{d x}=\frac{d u}{d x} v+u \frac{d v}{d x}
$$

where $u=x^{p}-c x$ and $v=\frac{1}{\left[\left(x^{p}-c x\right)^{2}+\left(y^{p}-c y\right)^{2}+\left(z^{p}\right)^{2}\right]^{3 / 2}}$. This gives

$$
\begin{gathered}
\frac{\partial u_{p t, \text { source }}}{\partial x^{p}}=\frac{1}{\left[\left(x^{p}-c x\right)^{2}+\left(y^{p}-c y\right)^{2}+\left(z^{p}\right)^{2}\right]^{3 / 2}} \frac{d}{d x^{p}}\left(x^{p}-c x\right)+ \\
\left(x^{p}-c x\right) \frac{d}{d x^{p}}\left(\frac{1}{\left[\left(x^{p}-c x\right)^{2}+\left(y^{p}-c y\right)^{2}+\left(z^{p}\right)^{2}\right]^{3 / 2}}\right) .
\end{gathered}
$$


Since the derivative of a sum is the sum of the derivatives, Equation C.16 becomes

$$
\begin{gathered}
\frac{\partial u_{p t, \text { source }}}{\partial x^{p}}=\frac{\frac{d}{d x^{p}}(-a)+\frac{d}{d x}\left(x^{p}\right)}{\left[\left(x^{p}-c x\right)^{2}+\left(y^{p}-c y\right)^{2}+\left(z^{p}\right)^{2}\right]^{3 / 2}}+ \\
\left(x^{p}-c x\right) \frac{d}{d x^{p}}\left(\frac{1}{\left[\left(x^{p}-c x\right)^{2}+\left(y^{p}-c y\right)^{2}+\left(z^{p}\right)^{2}\right]^{3 / 2}}\right) .
\end{gathered}
$$

The derivative of a constant is 0 , and the derivative of $x^{p}$ with respect to itself is 1 ,

so

$$
\begin{gathered}
\frac{\partial u_{p t, \text { source }}}{\partial x^{p}}=\frac{1}{\left[\left(x^{p}-c x\right)^{2}+\left(y^{p}-c y\right)^{2}+\left(z^{p}\right)^{2}\right]^{3 / 2}}+ \\
\left(x^{p}-c x\right) \frac{d}{d x^{p}}\left(\frac{1}{\left[\left(x^{p}-c x\right)^{2}+\left(y^{p}-c y\right)^{2}+\left(z^{p}\right)^{2}\right]^{3 / 2}}\right) .
\end{gathered}
$$

Now, using the chain rule as follows, with dummy variables $u, v$, and $n$,

$$
\frac{d u^{n}}{d x}=n u^{n-1} \frac{d u}{d x},
$$

where, in this case, $u=\left(x^{p}-c x\right)^{2}+\left(y^{p}-c y\right)^{2}+(z)^{2}$ and $n=-(3 / 2)$ gives

$$
\begin{gathered}
\frac{\partial u_{p t, \text { source }}}{\partial x^{p}}=\frac{1}{\left[\left(x^{p}-c x\right)^{2}+\left(y^{p}-c y\right)^{2}+\left(z^{p}\right)^{2}\right]^{3 / 2}}- \\
\left(\frac{3\left(x^{p}-c x\right)}{2\left[\left(x^{p}-c x\right)^{2}+\left(y^{p}-c y\right)^{2}+\left(z^{p}\right)^{2}\right]^{5 / 2}}\right) \frac{d}{d x^{p}}\left(\left(x^{p}-c x\right)^{2}+\left(y^{p}-c y\right)^{2}+\left(z^{p}\right)^{2}\right) .
\end{gathered}
$$

Once again, the derivative of a sum is the sum of the derivatives, resulting in

$$
\begin{gathered}
\frac{\partial u_{p t, \text { source }}}{\partial x^{p}}=\frac{1}{\left[\left(x^{p}-c x\right)^{2}+\left(y^{p}-c y\right)^{2}+\left(z^{p}\right)^{2}\right]^{3 / 2}}- \\
\left(\frac{3\left(x^{p}-c x\right)\left[\frac{d}{d x^{p}}\left(x^{p}-c x\right)^{2}+\frac{d}{d x^{p}}\left(y^{p}-c y\right)^{2}+\frac{d}{d x^{p}}\left(z^{p}\right)^{2}\right]}{2\left[\left(x^{p}-c x\right)^{2}+\left(y^{p}-c y\right)^{2}+\left(z^{p}\right)^{2}\right]^{5 / 2}}\right) .
\end{gathered}
$$

Again using the chain rule from Equation C.19, this time with $u=\left(x^{p}-c x\right)$ and $n=2$, and noting that, since both $\left(y^{p}-c y\right)^{2}$ and $(z)^{2}$ aren't dependent on $x^{p}$ the 
derivative of each is zero, Equation C.21 becomes

$$
\begin{gathered}
\frac{\partial u_{p t, \text { source }}}{\partial x^{p}}=\frac{1}{\left[\left(x^{p}-c x\right)^{2}+\left(y^{p}-c y\right)^{2}+\left(z^{p}\right)^{2}\right]^{3 / 2}}- \\
\left(\frac{3\left(x^{p}-c x\right)\left[2\left(x^{p}-c x\right) \frac{d}{d x^{p}}\left(x^{p}-c x\right)\right]}{2\left[\left(x^{p}-c x\right)^{2}+\left(y^{p}-c y\right)^{2}+\left(z^{p}\right)^{2}\right]^{5 / 2}}\right) .
\end{gathered}
$$

Using the fact that the derivative of a sum is the sum of the derivatives, and that the derivative of $x^{p}$ is 1 and the derivative of $c x$ is 0 , we now have

$$
\begin{gathered}
\frac{\partial u_{p t, \text { source }}}{\partial x^{p}}=\frac{1}{\left[\left(x^{p}-c x\right)^{2}+\left(y^{p}-c y\right)^{2}+\left(z^{p}\right)^{2}\right]^{3 / 2}}- \\
\left(\frac{3\left(x^{p}-c x\right) 2\left(x^{p}-c x\right)}{2\left[\left(x^{p}-c x\right)^{2}+\left(y^{p}-c y\right)^{2}+\left(z^{p}\right)^{2}\right]^{5 / 2}}\right) .
\end{gathered}
$$

Simplifying, by canceling the 2 on the top and bottom of the second term, and combining the two $\left(x^{p}-c x\right)$ terms, results in

$$
\begin{gathered}
\frac{\partial u_{p t, \text { source }}}{\partial x^{p}}=\frac{1}{\left[\left(x^{p}-c x\right)^{2}+\left(y^{p}-c y\right)^{2}+\left(z^{p}\right)^{2}\right]^{3 / 2}}- \\
\frac{3\left(x^{p}-c x\right)^{2}}{\left[\left(x^{p}-c x\right)^{2}+\left(y^{p}-c y\right)^{2}+\left(z^{p}\right)^{2}\right]^{5 / 2}} .
\end{gathered}
$$

Multiplying the entire equation by $s u b=\left[\left(x^{p}-c x\right)^{2}+\left(y^{p}-c y\right)^{2}+\left(z^{p}\right]^{2}\right)$ divided by itself, the equation can be simplified through common denominators, starting with

$$
\begin{gathered}
\frac{\partial u_{p t, \text { source }}}{\partial x^{p}}=\frac{s u b}{s u b}\left(\frac{1}{\left[\left(x^{p}-c x\right)^{2}+\left(y^{p}-c y\right)^{2}+\left(z^{p}\right)^{2}\right]^{3 / 2}}-\right. \\
\left.\frac{3\left(x^{p}-c x\right)^{2}}{\left[\left(x^{p}-c x\right)^{2}+\left(y^{p}-c y\right)^{2}+\left(z^{p}\right)^{2}\right]^{5 / 2}}\right) .
\end{gathered}
$$

Since the $s u b$ term is identically one, it can be applied to the first term in the parenthesis, but canceled out in the second term where it isn't needed, resulting in

$$
\frac{\partial u_{p t, \text { source }}}{\partial x^{p}}=\frac{s u b}{s u b\left[\left(x^{p}-c x\right)^{2}+\left(y^{p}-c y\right)^{2}+\left(z^{p}\right)^{2}\right]^{3 / 2}}-
$$




$$
\frac{3\left(x^{p}-c x\right)^{2}}{\left[\left(x^{p}-c x\right)^{2}+\left(y^{p}-c y\right)^{2}+\left(z^{p}\right)^{2}\right]^{5 / 2}},
$$

which raises the denominator of the first term in the equation to the $5 / 2$ power rather than the $3 / 2$, and thereby allows for the combining of the two terms into the final form of the equation, which can be written as

$$
\frac{\partial u_{p t, \text { source }}}{\partial x^{p}}=\frac{\left.\left(x^{p}-c x\right)^{2}+\left(y^{p}-c y\right)^{2}+\left(z^{p}\right)^{2}\right)-\left(3\left(x^{p}-c x\right)^{2}\right)}{\left[\left(x^{p}-c x\right)^{2}+\left(y^{p}-c y\right)^{2}+\left(z^{p}\right)^{2}\right]^{5 / 2}} .
$$

The equations for $\frac{\partial v_{p t, s o u r c e}}{\partial y^{p}}$ and $\frac{\partial w_{p t, \text { source }}}{\partial z^{p}}$ follow similar steps, and result in the equations

$$
\frac{\partial v_{p t, \text { source }}}{\partial y^{p}}=\frac{\left[\left(x^{p}-c x\right)^{2}+\left(y^{p}-c y\right)^{2}+\left(z^{p}\right)^{2}\right]-\left(3\left(y^{p}-c y\right)^{2}\right)}{\left[\left(x^{p}-c x\right)^{2}+\left(y^{p}-c y\right)^{2}+\left(z^{p}\right)^{2}\right]^{5 / 2}} .
$$

and

$$
\frac{\partial w_{p t, \text { source }}}{\partial z^{p}}=\frac{\left[\left(x^{p}-c x\right)^{2}+\left(y^{p}-c y\right)^{2}+\left(z^{p}\right)^{2}\right]-\left(3\left(z^{p}\right)^{2}\right)}{\left[\left(x^{p}-c x\right)^{2}+\left(y^{p}-c y\right)^{2}+\left(z^{p}\right)^{2}\right]^{5 / 2}} .
$$

All the remaining derivatives for the point source follow a similar pattern. As an example of how to find these derivatives, the calculation of $\frac{\partial u_{p t, s o u r c e}}{\partial y^{p}}$ will be examined. Since the numerator in the velocity influence equation is a constant with respect to $y^{p}$, it can be pulled out of the derivative, resulting in

$$
\frac{\partial u_{p t, \text { source }}}{\partial y^{p}}=\left(x^{p}-c x\right) \frac{d}{d y^{p}}\left(\frac{1}{\left[\left(x^{p}-c x\right)^{2}+\left(y^{p}-c y\right)^{2}+\left(z^{p}\right)^{2}\right]^{3 / 2}}\right) .
$$

Using the chain rule from Equation C.19, now with $u=\left(x^{p}-c x\right)^{2}+\left(y^{p}-c y\right)^{2}+\left(z^{p}\right)^{2}$ and $n=-(3 / 2)$ results in

$$
\frac{\partial u_{p t, \text { source }}}{\partial y^{p}}=\left(\frac{-3\left(x^{p}-c x\right) \frac{d}{d y^{p}}\left[\left(x^{p}-c x\right)^{2}+\left(y^{p}-c y\right)^{2}+\left(z^{p}\right)^{2}\right]}{2\left[\left(x^{p}-c x\right)^{2}+\left(y^{p}-c y\right)^{2}+\left(z^{p}\right)^{2}\right]^{5 / 2}}\right) .
$$


Applying the chain rule again, now with $u=y^{p}-c y$ and $n=2$, and simplifying since the derivative of $\left(x^{p}-c x\right)^{2}$ and $\left(z^{p}\right)^{2}$ with respect to $y^{p}$ are both 0 results in

$$
\frac{\partial u_{p t, \text { source }}}{\partial y^{p}}=\left(\frac{-3\left(x^{p}-c x\right)\left[2\left(y^{p}-c y\right) \frac{d}{d y^{p}}\left(y^{p}-c y\right)\right]}{2\left[\left(x^{p}-c x\right)^{2}+\left(y^{p}-c y\right)^{2}+\left(z^{p}\right)^{2}\right]^{5 / 2}}\right) .
$$

Recalling that the derivative of $\left(x^{p}-c x\right)$ with respect to $x^{p}$ was 1 , it is easy to show that the derivative of $\left(y^{p}-c y\right)$ with respect to $y^{p}$ is also 1 , and, after canceling the 2 in the numerator and denominator, this leads to the final equation

$$
\frac{\partial u_{p t, \text { source }}}{\partial y^{p}}=\left(\frac{-3\left(x^{p}-c x\right)\left(y^{p}-c y\right)}{\left[\left(x^{p}-c x\right)^{2}+\left(y^{p}-c y\right)^{2}+\left(z^{p}\right)^{2}\right]^{5 / 2}}\right) .
$$

Applying these same steps the remaining derivatives are

$$
\begin{aligned}
\frac{\partial u_{p t, \text { source }}}{\partial z^{p}} & =\left(\frac{-3\left(x^{p}-c x\right)\left(z^{p}-c z\right)}{\left[\left(x^{p}-c x\right)^{2}+\left(y^{p}-c y\right)^{2}+\left(z^{p}\right)^{2}\right]^{5 / 2}}\right), \\
\frac{\partial v_{p t, \text { source }}}{\partial x^{p}} & =\left(\frac{-3\left(y^{p}-c y\right)\left(x^{p}-c x\right)}{\left[\left(x^{p}-c x\right)^{2}+\left(y^{p}-c y\right)^{2}+\left(z^{p}\right)^{2}\right]^{5 / 2}}\right), \\
\frac{\partial v_{p t, \text { source }}}{\partial z^{p}} & =\left(\frac{-3\left(y^{p}-c y\right)\left(z^{p}-c z\right)}{\left[\left(x^{p}-c x\right)^{2}+\left(y^{p}-c y\right)^{2}+\left(z^{p}\right)^{2}\right]^{5 / 2}}\right), \\
\frac{\partial w_{p t, \text { source }}}{\partial x^{p}} & =\left(\frac{-3\left(z^{p}-c z\right)\left(x^{p}-c x\right)}{\left[\left(x^{p}-c x\right)^{2}+\left(y^{p}-c y\right)^{2}+\left(z^{p}\right)^{2}\right]^{5 / 2}}\right),
\end{aligned}
$$

and

$$
\frac{\partial w_{p t, \text { source }}}{\partial y^{p}}=\left(\frac{-3\left(z^{p}-c z\right)\left(y^{p}-c y\right)}{\left[\left(x^{p}-c x\right)^{2}+\left(y^{p}-c y\right)^{2}+\left(z^{p}\right)^{2}\right]^{5 / 2}}\right) .
$$

\section{C.1.2 Point Doublet}

The velocity influence equations for a point doublet are

$$
u_{p t, \text { doublet }}=\frac{3 \mu A}{4 \pi} \frac{\left(x-x_{0}\right)(z)}{4 \pi\left[\left(x-x_{0}\right)^{2}+\left(y-y_{0}\right)^{2}+(z)^{2}\right]^{5 / 2}},
$$




$$
v_{p t, \text { doublet }}=\frac{3 \mu A}{4 \pi} \frac{\left(y-y_{0}\right)(z)}{4 \pi\left[\left(x-x_{0}\right)^{2}+\left(y-y_{0}\right)^{2}+(z)^{2}\right]^{5 / 2}},
$$

and

$$
w_{p t, \text { doublet }}=\frac{-\mu A}{4 \pi} \frac{\left(x-x_{0}\right)^{2}+\left(y-y_{0}\right)^{2}-2(z)^{2}}{4 \pi\left[\left(x-x_{0}\right)^{2}+\left(y-y_{0}\right)^{2}+(z)^{2}\right]^{5 / 2}},
$$

where $\mu$ is the point doublet strength, $A$ is still the panel area, and $\left(x_{0}, y_{0}, z_{0}\right)$ and $(x, y, z)$ are still the element location and survey point respectively. Once again, the derivative of each of these equations must be taken with respect to each axis direction, resulting in nine derivatives for the point doublet element. Also like the previous point source section, there are patterns that emerge in the derivatives for the point doublet that prevent the need to fully calculate each equation individually. Again, $(x, y, z)$ will become $\left(x^{p}, y^{p}, z^{p}\right)$, and $\left(x_{0}, y_{0}, z_{0}\right)$ will become $(c x, c y, c z)$.

First, examining the derivative of Equation C.39 with respect to $x^{p}$, we use the product rule as stated in Equation C.15, where $u=\left(x^{p}-c x\right)$ and $v=\frac{1}{\left[\left(x^{p}-c x\right)^{2}+\left(y^{p}-c y\right)^{2}+(z)^{2}\right]^{5 / 2}}$, which results in

$$
\begin{aligned}
& \frac{\partial u_{p t, \text { doublet }}}{\partial x^{p}}=3 z\left(\frac{1}{\left[\left(x^{p}-c x\right)^{2}+\left(y^{p}-c y\right)^{2}+(z)^{2}\right]^{5 / 2}} \frac{d}{d x}\left(x^{p}-c x\right)+\right. \\
&\left.\left(x^{p}-c x\right) \frac{d}{d x}\left(\frac{1}{\left[\left(x^{p}-c x\right)^{2}+\left(y^{p}-c y\right)^{2}+(z)^{2}\right]^{5 / 2}}\right)\right) .
\end{aligned}
$$

Since the derivative of a sum is the sum of the derivatives, and the derivative of $c x$ with respect to $x^{p}$ is 0 , while the derivative of $x^{p}$ with respect to itself is 1 , this becomes

$$
\frac{\partial u_{p t, \text { doublet }}}{\partial x^{p}}=3 z\left(\frac{1}{\left[\left(x^{p}-c x\right)^{2}+\left(y^{p}-c y\right)^{2}+(z)^{2}\right]^{5 / 2}}+\right.
$$




$$
\left.\left(x^{p}-c x\right) \frac{d}{d x}\left(\frac{1}{\left[\left(x^{p}-c x\right)^{2}+\left(y^{p}-c y\right)^{2}+(z)^{2}\right]^{5 / 2}}\right)\right) .
$$

Moving on to the second derivative term in the equation, again applying the chain rule, now with $u=\left(x^{p}-c x\right)^{2}+\left(y^{p}-c y\right)^{2}+(z)^{2}$ and $n=-5 / 2$, results in

$$
\begin{gathered}
\frac{\partial u_{p t, \text { doublet }}}{\partial x^{p}}=3 z\left(\frac{1}{\left[\left(x^{p}-c x\right)^{2}+\left(y^{p}-c y\right)^{2}+(z)^{2}\right]^{5 / 2}}-\right. \\
\left.\left(\frac{5\left(x^{p}-c x\right)}{2\left[\left(x^{p}-c x\right)^{2}+\left(y^{p}-c y\right)^{2}+(z)^{2}\right]^{7 / 2}}\right) \frac{d}{d x}\left(\left(x^{p}-c x\right)^{2}+\left(y^{p}-c y\right)^{2}+(z)^{2}\right)\right) .
\end{gathered}
$$

Once again the derivative of a sum is the sum of the derivatives, and the derivatives with respect to $x^{p}$ of both $\left(y^{p}-c y\right)^{2}$ and $(z)^{2}$ are zero, so

$$
\begin{gathered}
\frac{\partial u_{p t, \text { doublet }}}{\partial x^{p}}=3 z\left(\frac{1}{\left[\left(x^{p}-c x\right)^{2}+\left(y^{p}-c y\right)^{2}+(z)^{2}\right]^{5 / 2}}-\right. \\
\left.\left(\frac{5\left(x^{p}-c x\right) \frac{d}{d x}\left(\left(x^{p}-c x\right)^{2}\right)}{2\left[\left(x^{p}-c x\right)^{2}+\left(y^{p}-c y\right)^{2}+(z)^{2}\right]^{7 / 2}}\right)\right) .
\end{gathered}
$$

Using the chain rule with $u=\left(x^{p}-c x\right)$ and $n=2$ leads to

$$
\begin{gathered}
\frac{\partial u_{p t, \text { doublet }}}{\partial x^{p}}=3 z\left(\frac{1}{\left[\left(x^{p}-c x\right)^{2}+\left(y^{p}-c y\right)^{2}+(z)^{2}\right]^{5 / 2}}-\right. \\
\left.\left(\frac{5\left(x^{p}-c x\right) 2\left(x^{p}-c x\right) \frac{d}{d x}\left(x^{p}-c x\right)}{2\left[\left(x^{p}-c x\right)^{2}+\left(y^{p}-c y\right)^{2}+(z)^{2}\right]^{7 / 2}}\right)\right) .
\end{gathered}
$$

Applying the fact that the derivative of a sum is the sum of the derivatives, and that the derivative of $c x$ with respect to $x^{p}$ is zero, and canceling the twos in the numerator and denominator results in

$$
\begin{gathered}
\frac{\partial u_{p t, \text { doublet }}}{\partial x^{p}}=3 z\left(\frac{1}{\left[\left(x^{p}-c x\right)^{2}+\left(y^{p}-c y\right)^{2}+(z)^{2}\right]^{5 / 2}}-\right. \\
\left.\left(\frac{5\left(x^{p}-c x\right)^{2}}{\left[\left(x^{p}-c x\right)^{2}+\left(y^{p}-c y\right)^{2}+(z)^{2}\right]^{7 / 2}}\right)\right) .
\end{gathered}
$$


Combining the two terms in the parentheses using the same philosophy as in Equation C.25 to obtain a common denominator results in

$$
\frac{\partial u_{p t, d o u b l e t}}{\partial x^{p}}=3 z\left(\frac{\left[\left(x^{p}-c x\right)^{2}+\left(y^{p}-c y\right)^{2}+(z)^{2}\right]-\left(5\left(x^{p}-c x\right)^{2}\right)}{\left[\left(x^{p}-c x\right)^{2}+\left(y^{p}-c y\right)^{2}+(z)^{2}\right]^{7 / 2}}\right) .
$$

The equation for $\frac{\partial v_{p t, \text { doublet }}}{\partial y^{p}}$ can be found following similar steps. The final equation for that term can be written as

$$
\frac{\partial v_{p t, \text { doublet }}}{\partial y^{p}}=3 z\left(\frac{\left[\left(x^{p}-c x\right)^{2}+\left(y^{p}-c y\right)^{2}+(z)^{2}\right]-\left(5\left(y^{p}-c y\right)^{2}\right)}{\left[\left(x^{p}-c x\right)^{2}+\left(y^{p}-c y\right)^{2}+(z)^{2}\right]^{7 / 2}}\right) .
$$

Also similar are the equations for $\frac{\partial u_{p t, d o u b l e t}}{\partial z^{p}}$ and $\frac{\partial v_{p t, d o u b l e t}}{\partial z^{p}}$, which can be written as

$$
\frac{\partial u_{p t, \text { doublet }}}{\partial z^{p}}=3\left(x^{p}-c x\right)\left(\frac{\left[\left(x^{p}-c x\right)^{2}+\left(y^{p}-c y\right)^{2}+(z)^{2}\right]-\left(5\left(z^{p}\right)^{2}\right)}{\left[\left(x^{p}-c x\right)^{2}+\left(y^{p}-c y\right)^{2}+(z)^{2}\right]^{7 / 2}}\right),
$$

and

$$
\frac{\partial v_{p t, \text { doublet }}}{\partial z^{p}}=3\left(y^{p}-c y\right)\left(\frac{\left[\left(x^{p}-c x\right)^{2}+\left(y^{p}-c y\right)^{2}+(z)^{2}\right]-\left(5\left(z^{p}\right)^{2}\right)}{\left[\left(x^{p}-c x\right)^{2}+\left(y^{p}-c y\right)^{2}+(z)^{2}\right]^{7 / 2}}\right) .
$$

The derivatives for $\frac{\partial u_{p t, d o u b l e t}}{\partial y^{p}}$ and $\frac{\partial v_{p t, \text { doublet }}}{\partial x^{p}}$ can each be found using similar procedures. The derivation for $\frac{\partial u_{p t, \text { doublet }}}{\partial y^{p}}$ will be examined to show the necessary steps. The first step is to pull out the constant numerator, and take the derivative of the remaining fraction using the chain rule.

$$
\frac{\partial u_{p t, \text { doublet }}}{\partial y^{p}}=\frac{3\left(x^{p}-c x\right)\left(z^{p}\right) \mu A}{4 \pi} \frac{d}{d y^{p}}\left(\frac{1}{\left[\left(x^{p}-c x\right)^{2}+\left(y^{p}-c y\right)^{2}+\left(z^{p}\right)^{2}\right]^{5 / 2}}\right) .
$$

The chain rule is applied with $u=\left(x^{p}-c x\right)^{2}+\left(y^{p}-c y\right)^{2}+(z)^{2}$ and $n=-5 / 2$ giving

$$
\frac{\partial u_{p t, d o u b l e t}}{\partial y^{p}}=\frac{\mu A}{4 \pi}\left(\frac{-15\left(z^{p}\right)\left(x^{p}-c x\right) \frac{d}{d y^{p}}\left(\left(x^{p}-c x\right)^{2}+\left(y^{p}-c y\right)^{2}+\left(z^{p}\right)^{2}\right)}{2\left[\left(x^{p}-c x\right)^{2}+\left(y^{p}-c y\right)^{2}+\left(z^{p}\right)^{2}\right]^{7 / 2}}\right) .
$$


The remaining derivative term has been taking several times already in previous derivations, where first the derivative of the sum is broken into the sum of the derivatives, then the constant terms whose derivatives are zero are removed, and the chain rule is applied on the $\left(y^{p}-c y\right)^{2}$ term. These steps result in

$$
\frac{\partial u_{p t, \text { doublet }}}{\partial y^{p}}=\frac{-\mu A}{4 \pi} \frac{15\left(z^{p}\right)\left(x^{p}-c x\right)\left(y^{p}-c y\right)}{\left[\left(x^{p}-c x\right)^{2}+\left(y^{p}-c y\right)^{2}+\left(z^{p}\right)^{2}\right]^{7 / 2}},
$$

and, similarly,

$$
\frac{\partial v_{p t, \text { doublet }}}{\partial x^{p}}=\frac{-\mu A}{4 \pi} \frac{15\left(z^{p}\right)\left(x^{p}-c x\right)\left(y^{p}-c y\right)}{\left[\left(x^{p}-c x\right)^{2}+\left(y^{p}-c y\right)^{2}+\left(z^{p}\right)^{2}\right]^{7 / 2}} .
$$

The only remaining derivatives to be found for the point doublet involve the $w_{p t, \text { doublet }}$ equation. The derivatives of this with respect to $x^{p}$ and $y^{p}$ follow identical steps, and the derivative with respect to $x^{p}$ will be examined in detail. The first step is to use the product rule, where $u=\left(x^{p}-c x\right)^{2}+\left(y^{p}-c y\right)^{2}-2(z)^{2}$ and $v=\frac{1}{\left[\left(x^{p}-c x\right)^{2}+\left(y^{p}-c y\right)^{2}+\left(z^{p}\right)^{2}\right]^{5 / 2}}$, which results in

$$
\begin{gathered}
\frac{\partial w_{p t, d o u b l e t}}{\partial x^{p}}=\frac{-\frac{d}{d x}\left(\left(x^{p}-c x\right)^{2}+\left(y^{p}-c y\right)^{2}-2(z)^{2}\right)}{\left[\left(x^{p}-c x\right)^{2}+\left(y^{p}-c y\right)^{2}+\left(z^{p}\right)^{2}\right]^{5 / 2}}- \\
\left(\left(x^{p}-c x\right)^{2}+\left(y^{p}-c y\right)^{2}-2(z)^{2}\right) \frac{d}{d x}\left(\frac{1}{\left[\left(x^{p}-c x\right)^{2}+\left(y^{p}-c y\right)^{2}+\left(z^{p}\right)^{2}\right]^{5 / 2}}\right) .
\end{gathered}
$$

The derivative of a sum is the sum of the derivatives, and the derivatives of $y^{p}, c y$, and $z^{p}$ with respect to $x^{p}$ are all zero, resulting in

$$
\begin{gathered}
\frac{\partial w_{p t, \text { doublet }}}{\partial x^{p}}=\frac{-\frac{d}{d x}\left(\left(x^{p}-c x\right)^{2}\right)}{\left[\left(x^{p}-c x\right)^{2}+\left(y^{p}-c y\right)^{2}+\left(z^{p}\right)^{2}\right]^{5 / 2}}- \\
{\left[\left(x^{p}-c x\right)^{2}+\left(y^{p}-c y\right)^{2}-2(z)^{2}\right] \frac{d}{d x}\left(\frac{1}{\left[\left(x^{p}-c x\right)^{2}+\left(y^{p}-c y\right)^{2}+\left(z^{p}\right)^{2}\right]^{5 / 2}}\right) .}
\end{gathered}
$$


Now using the chain rule, with $u=\left(x^{p}-c x\right)^{2}+\left(y^{p}-c y\right)^{2}-2(z)^{2}$ and $n=-5 / 2$, results in

$$
\begin{gathered}
\frac{\partial w_{p t, \text { doublet }}}{\partial x^{p}}=\frac{-\frac{d}{d x}\left(\left(x^{p}-c x\right)^{2}\right)}{\left[\left(x^{p}-c x\right)^{2}+\left(y^{p}-c y\right)^{2}+\left(z^{p}\right)^{2}\right]^{5 / 2}}- \\
\frac{5\left[-\left(x^{p}-c x\right)^{2}-\left(y^{p}-c y\right)^{2}+2(z)^{2}\right] \frac{d}{d x}\left(\left[\left(x^{p}-c x\right)^{2}+\left(y^{p}-c y\right)^{2}+(z)^{2}\right]\right.}{2\left[\left(x^{p}-c x\right)^{2}+\left(y^{p}-c y\right)^{2}+\left(z^{p}\right)^{2}\right]^{7 / 2}} .
\end{gathered}
$$

Once again the derivatives of $y^{p}, c y$, and $z^{p}$ with respect to $x^{p}$ are all zero, so those terms can be removed. Additionally, the derivative of $\left(x^{p}-c x\right)^{2}$, which appears in the equation twice, can be found using the chain rule, with $u=x^{p}-c x$ and $n=2$, resulting in

$$
\begin{gathered}
\frac{\partial w_{p t, \text { doublet }}}{\partial x^{p}}=\frac{-2\left(x^{p}-c x\right) \frac{d}{d x}\left(\left(x^{p}-c x\right)\right)}{\left[\left(x^{p}-c x\right)^{2}+\left(y^{p}-c y\right)^{2}+\left(z^{p}\right)^{2}\right]^{5 / 2}}- \\
\frac{5\left[-\left(x^{p}-c x\right)^{2}-\left(y^{p}-c y\right)^{2}+2(z)^{2}\right] 2\left(x^{p}-c x\right) \frac{d}{d x}\left(\left(x^{p}-c x\right)\right)}{2\left[\left(x^{p}-c x\right)^{2}+\left(y^{p}-c y\right)^{2}+\left(z^{p}\right)^{2}\right]^{7 / 2}} .
\end{gathered}
$$

The derivative of $c x$ with respect to $x^{p}$ is zero, and the derivative of $x^{p}$ with respect to itself is one, so the previous equation can be rewritten as

$$
\begin{gathered}
\frac{\partial w_{p t, \text { doublet }}}{\partial x^{p}}=\frac{-2\left(x^{p}-c x\right)}{\left[\left(x^{p}-c x\right)^{2}+\left(y^{p}-c y\right)^{2}+\left(z^{p}\right)^{2}\right]^{5 / 2}}- \\
\frac{5\left[-\left(x^{p}-c x\right)^{2}-\left(y^{p}-c y\right)^{2}+2(z)^{2}\right]\left(x^{p}-c x\right)}{\left[\left(x^{p}-c x\right)^{2}+\left(y^{p}-c y\right)^{2}+\left(z^{p}\right)^{2}\right]^{7 / 2}} .
\end{gathered}
$$

Using the same technique applied in Equation C.25 to obtain a common denominator, this result can be simplified to

$$
\frac{\partial w_{p t, \text { doublet }}}{\partial x^{p}}=\frac{\left[3\left(x^{p}-c x\right)^{2}+3\left(y^{p}-c y\right)^{2}-12(z)^{2}\right]\left(x^{p}-c x\right)}{\left[\left(x^{p}-c x\right)^{2}+\left(y^{p}-c y\right)^{2}+\left(z^{p}\right)^{2}\right]^{7 / 2}} .
$$

The equation for $\frac{\partial w_{p t, \text { doublet }}}{\partial y^{p}}$ can be found in a similar manner, and its final form is

$$
\frac{\partial w_{p t, \text { doublet }}}{\partial y^{p}}=\frac{\left[3\left(x^{p}-c x\right)^{2}+3\left(y^{p}-c y\right)^{2}-12(z)^{2}\right]\left(y^{p}-c y\right)}{\left[\left(x^{p}-c x\right)^{2}+\left(y^{p}-c y\right)^{2}+\left(z^{p}\right)^{2}\right]^{7 / 2}} .
$$




\section{C.1.3 Constant Strength Source}

The velocity influence equation for a constant strength source panel with four sides can be written in vector form as

$$
\vec{u}_{\text {const }, \text { source }}=\sum_{i=1}^{4} \mathbf{G L}(\mathbf{S M}(\mathbf{l})-\mathbf{S L}(\mathbf{m}))+C_{J K, i}(\mathbf{n}) .
$$

Each of these terms is defined in detail in the VSAERO theory document. 68

The simplest way to construct the necessary derivative terms of this equation is to symbolically solve the derivative of Equation C.63, keeping in mind which terms in the equation are functions of the term for which the derivative is being taken. This allows for a symbolic derivative equation that is made up of a series of simple derivative terms that can easily be found to construct a complete analytic derivative equation. To start, the symbolic derivative of Equation C.63 will be found, in this case with respect to $x^{p}$. The first step is to pull the sum outside the derivative, since the derivative of a sum is the sum of the derivatives, which results in

$$
\frac{\partial \vec{u}_{\text {const }, \text { source }}}{\partial x^{p}}=\sum_{i=1}^{4} \frac{\partial}{\partial x^{p}} \mathbf{G L}(\mathbf{S M}(\mathbf{l})-\mathbf{S L}(\mathbf{m}))+C_{J K, i}(\mathbf{n})
$$

Since the derivative of a sum or difference is the sum or difference of that derivative, the derivative can be distributed, and the equation can be rewritten as

$$
\frac{\partial \vec{u}_{\text {const }, \text { source }}}{\partial x^{p}}=\sum_{i=1}^{4}\left(\frac{\partial}{\partial x^{p}}(\mathbf{G L ~ S M}(\mathbf{l}))-\frac{\partial}{\partial x^{p}}(\mathbf{G L ~ S L}(\mathbf{m}))\right)+\frac{\partial}{\partial x^{p}}\left(C_{J K, i}(\mathbf{n})\right) .
$$

Since the panel coordinate vectors, $\mathbf{l}, \mathbf{m}$, and $\mathbf{n}$ are not dependent on any of the survey 
point locations, those vector values can be taken out of the derivatives, leaving

$$
\frac{\partial \vec{u}_{\text {const }, \text { source }}}{\partial x^{p}}=\sum_{i=1}^{4}\left((\mathbf{l}) \frac{\partial}{\partial x^{p}}(\mathbf{G L ~ S M})-(\mathbf{m}) \frac{\partial}{\partial x^{p}}(\mathbf{G L ~ S L})\right)+(\mathbf{n}) \frac{\partial}{\partial x^{p}}\left(C_{J K, i}\right) .
$$

Finally, the product rule can be applied to both GL SL and GL SM to achieve the final symbolic form of the derivative.

$$
\begin{gathered}
\frac{\partial \vec{u}_{\text {const }, \text { source }}}{\partial x^{p}}=\sum_{i=1}^{4}\left((\mathbf{l})\left(\left(\frac{\partial}{\partial x^{p}}(\mathbf{G L}) \mathbf{S M}\right)+\left(\mathbf{G L} \frac{\partial}{\partial x^{p}}(\mathbf{S M})\right)\right)-\right. \\
(\mathbf{m})\left(\left(\frac{\partial}{\partial x^{p}}(\mathbf{G L}) \mathbf{S L}\right)+\left(\mathbf{G L} \frac{\partial}{\partial x^{p}}(\mathbf{S L})\right)\right)+(\mathbf{n}) \frac{\partial}{\partial x^{p}}\left(C_{J K, i}\right) .
\end{gathered}
$$

Examining Equation C.67 shows that four derivatives are necessary to quantitatively calculate complete the overall velocity derivative, and these four derivatives are $\frac{\partial \mathbf{G L}}{\partial x^{p}}, \frac{\partial \mathbf{S M}}{\partial x^{p}}, \frac{\partial \mathbf{S L}}{\partial x^{p}}$, and $\frac{\partial C_{j k}}{\partial x^{p}}$. Each of the terms being differentiated are in turn functions of $x^{p}$, and so each derivative must be examined in turn. First, $\frac{\partial \mathbf{G L}}{\partial x^{p}}$ will be derived, where GL is defined as

$$
\mathbf{G L}=\frac{1}{s} \log \left(\frac{A+B+s}{A+B-s}\right)
$$

where $s=|\mathbf{s}|=|\mathbf{b}-\mathbf{a}|, A=|\mathbf{a}|$, and $B=|\mathbf{b}|$. The first step to finding the derivative of this is to pull the $1 / s$ term outside the derivative, since it is not a function of $x^{p}$, and then the chain rule must be employed, with $u=x^{2}$ and $\frac{\partial \log (w)}{\partial x}=\frac{1}{u}$, resulting in

$$
\frac{\partial \mathbf{G L}}{\partial x^{p}}=\frac{1}{s}\left[\frac{1}{\left(\frac{A+B+s}{A+B-s}\right)} \frac{\partial}{\partial x^{p}}\left(\frac{A+B+s}{A+B-s}\right)\right] .
$$

The product rule in Equation C.15 can then be applied to the derivative term, and the fraction can be inverted, which results in

$$
\frac{\partial \mathbf{G} \mathbf{L}}{\partial x^{p}}=\frac{1}{s}\left[\frac { A + B - s } { A + B + s } \left(\frac{\partial}{\partial x^{p}}(A+B+s)\left[\frac{1}{A+B-s}\right]+\right.\right.
$$




$$
\left.\left.\left((A+B+s) \frac{\partial}{\partial x^{p}}\left[\frac{1}{A+B-s}\right]\right)\right)\right] .
$$

Since the derivative of a sum is the sum of the derivatives, and $s$ isn't a function of $x^{p}$, this can be reduced to

$$
\begin{gathered}
\frac{\partial \mathbf{G L}}{\partial x^{p}}=\frac{1}{s}\left[\frac { A + B - s } { A + B + s } \left(\left(\frac{\partial}{\partial x^{p}}(A)+\frac{\partial}{\partial x^{p}}(B)\right)\left[\frac{1}{A+B-s}\right]+\right.\right. \\
\left.\left.\left((A+B+s) \frac{\partial}{\partial x^{p}}\left[\frac{1}{A+B-s}\right]\right)\right)\right]
\end{gathered}
$$

The quotient rule can then be applied to the derivative in the second term, resulting in a required derivative equal to the one calculated in the previous step,

$$
\begin{aligned}
\frac{\partial \mathbf{G L}}{\partial x^{p}}=\frac{1}{s} & {\left[\frac { A + B - s } { A + B + s } \left(\left(\frac{\partial}{\partial x^{p}}(A)+\frac{\partial}{\partial x^{p}}(B)\right)\left[\frac{1}{A+B-s}\right]+\right.\right.} \\
& \left.\left.\left((A+B+s)\left[\frac{-\frac{\partial}{\partial x^{p}}(A+B-s)}{(A+B-s)^{2}}\right]\right)\right)\right],
\end{aligned}
$$

which can be simplied in the exact same was as before. The final equation can be written as

$$
\begin{aligned}
\frac{\partial \mathbf{G L}}{\partial x^{p}}=\frac{1}{s} & {\left[\frac { A + B - s } { A + B + s } \left(\left(\frac{\partial}{\partial x^{p}}(A)+\frac{\partial}{\partial x^{p}}(B)\right)\left[\frac{1}{A+B-s}\right]+\right.\right.} \\
& \left.\left.\left((A+B+s)\left[\frac{\left(-\frac{\partial}{\partial x^{p}}(A)-\frac{\partial}{\partial x^{p}}(B)\right)}{(A+B-s)^{2}}\right]\right)\right)\right],
\end{aligned}
$$

Next, the derivative of $S M$ and $S L$ will be examined. Each of these terms is a function of the vector $\mathbf{s}$, and one of the panel coordinate vectors. Because neither $\mathbf{s}$, nor the panel coordinate vectors are dependent on the survey location, the derivative of each of these terms is identically zero.

$$
\frac{\partial S L}{\partial x^{p}}=\frac{\partial}{\partial x^{p}}(\mathbf{s} \cdot \mathbf{l})=\left(\frac{\partial}{\partial x^{p}}(\mathbf{s}) \cdot \mathbf{l}\right)+\left(\mathbf{s} \cdot \frac{\partial}{\partial x^{p}}(\mathbf{l})\right)=0
$$


and

$$
\frac{\partial S M}{\partial x^{p}}=\frac{\partial}{\partial x^{p}}(\mathbf{s} \cdot \mathbf{m})=\left(\frac{\partial}{\partial x^{p}}(\mathbf{s}) \cdot \mathbf{m}\right)+\left(\mathbf{s} \cdot \frac{\partial}{\partial x^{p}}(\mathbf{m})\right)=0
$$

One final term in Equation C.67 remains to be found to be found; $\frac{\partial C_{j k}}{\partial x^{p}}$. Much like Equation C.67, the derivative for this term must be done symbolically, and the resulting component derivatives must then be found to assemble the complete derivative term. The derivative becomes

$$
\frac{\partial C_{j k}}{\partial x^{p}}=\frac{\partial}{\partial x^{p}}\left(\operatorname{atan}\left(\frac{R N U M}{D N O M}\right)\right)
$$

where $R N U M=S M P N(B P A-A P B)$ and $D N O M=P A P B+\left(\left(P N^{2}\right) A B\left(S M^{2}\right)\right)$. Using the chain rule, with $u=\frac{R N U M}{D N O M}$ and $\frac{\partial \operatorname{atan}(u)}{\partial x}=\frac{1}{X^{2}+1}$, the derivative of $C_{j k}$ can be rewritten as

$$
\frac{\partial C_{j k}}{\partial x^{p}}=\frac{\frac{\partial}{\partial x^{p}}\left(\frac{R N U M}{D N O M}\right)}{\left(\frac{R N U M}{D N O M}\right)^{2}+1}
$$

Applying the product rule with $u=R N U M$ and $v=\frac{1}{D N O M}$ results in

$$
\frac{\partial C_{j k}}{\partial x^{p}}=\frac{\left(\frac{\partial}{\partial x^{p}}(R N U M) \frac{1}{D N O M}\right)+\left(R N U M \frac{\partial}{\partial x^{p}}\left(\frac{1}{D N O M}\right)\right)}{\left(\frac{R N U M}{D N O M}\right)^{2}+1} .
$$

The partial derivative of $\frac{1}{D N O M}$ can be simplified using the quotient rule, resulting in

$$
\frac{\partial C_{j k}}{\partial x^{p}}=\frac{\left(\left(\frac{\partial}{\partial x^{p}}(R N U M) \frac{1}{D N O M}\right)+\left(R N U M\left(\frac{-\frac{\partial}{\partial x^{p}} D N O M}{D N O M^{2}}\right)\right)\right)}{\left(\frac{R N U M}{D N O M}\right)^{2}+1}
$$

This means it is necessary to calculate the derivatives of $R N U M$ and $D N O M$. The derivation of each follows, starting with $R N U M$.

$$
\frac{\partial R N U M}{\partial x^{p}}=\frac{\partial}{\partial x^{p}}(S M P N(B P A-A P B))
$$


Using the product rule with $u=S M P N$ and $v=B P A-A P B$ results in

$$
\begin{gathered}
\frac{\partial R N U M}{\partial x^{p}}=\frac{\partial}{\partial x^{p}}(S M P N)(B P A-A P B)+ \\
(S M P N) \frac{\partial}{\partial x^{p}}(B P A-A P B) .
\end{gathered}
$$

The product rule can then be applied to the first derivative, and it has already been shown that the derivative of $S M$ is zero, and the derivative of the difference can be distributed, so the equation can be rewritten as

$$
\begin{gathered}
\frac{\partial R N U M}{\partial x^{p}}=S M \frac{\partial}{\partial x^{p}}(P N)(B P A-A P B)+ \\
(S M P N)\left(\frac{\partial}{\partial x^{p}}(B P A)-\frac{\partial}{\partial x^{p}}(A P B)\right) .
\end{gathered}
$$

Using the product rule a final time results in the final form of this symbolic derivative as follows

$$
\begin{gathered}
\frac{\partial R N U M}{\partial x^{p}}=S M \frac{\partial}{\partial x^{p}}(P N)(B P A-A P B)+ \\
(S M P N)\left(\left(\frac{\partial}{\partial x^{p}} B P A+B \frac{\partial}{\partial x^{p}}(P A)\right)-\left(\left(\frac{\partial}{\partial x^{p}}(A) P B+A \frac{\partial}{\partial x^{p}}(P B)\right)\right)\right) .
\end{gathered}
$$

This final form requires the derivative for $P N, A, B, P A$, and $P B$. The derivatives of $A$ and $B$ have already been found previously, so there are three derivatives that remain to be found. $P N$ will be examined first, and the initial derivative can be written

$$
\frac{\partial P N}{\partial x^{p}}=\frac{\partial}{\partial x^{p}}\left(\mathbf{P}_{j k} \cdot \mathbf{n}_{k}\right)
$$

which can be rewritten as

$$
\frac{\partial P N}{\partial x^{p}}=\frac{\partial}{\partial x^{p}}\left(\left[\left(x^{p}-c x\right) \hat{i}+\left(y^{p}-c y\right) \hat{j}+\left(z^{p}-c z\right) \hat{k}\right] \cdot \mathbf{n}_{k}\right) .
$$


The derivative term can be distributed into the dot product, and the second dot product vanishes because $n$ isn't dependent on $x^{p}$, resulting in

$$
\frac{\partial P N}{\partial x^{p}}=\left(\frac{\partial}{\partial x^{p}}\left[\left(x^{p}-c x\right) \hat{i}+\left(y^{p}-c y\right) \hat{j}+\left(z^{p}-c z\right) \hat{k}\right] \cdot \mathbf{n}_{k}\right) .
$$

This results in a single term in the position vector showing up, depending on what the derivative is taken with respect to, which is then dotted with the corresponding term in the normal vector, resulting in the following equations for each axis

$$
\begin{aligned}
& \frac{\partial P N}{\partial x^{p}}=n_{x}, \\
& \frac{\partial P N}{\partial y^{p}}=n_{y},
\end{aligned}
$$

and

$$
\frac{\partial P N}{\partial z^{p}}=n_{z}
$$

Next, the derivatives of $P A$ will be examined, and the starting point is

$$
\frac{\partial P A}{\partial x^{p}}=\frac{\partial}{\partial x^{p}}(\mathbf{a} \cdot(\mathbf{l} \times(\mathbf{a} \times \mathbf{s})))
$$

which, through the properties of dot products, becomes

$$
\frac{\partial P A}{\partial x^{p}}=\left[\frac{\partial}{\partial x^{p}}(\mathbf{a}) \cdot(\mathbf{l} \times(\mathbf{a} \times \mathbf{s}))\right]+\left[\mathbf{a} \cdot \frac{\partial}{\partial x^{p}}(\mathbf{l} \times(\mathbf{a} \times \mathbf{s}))\right] .
$$

Now, using the properties of cross products, the second term can be rewritten, resulting in the equation

$$
\frac{\partial P A}{\partial x^{p}}=\left[\frac{\partial}{\partial x^{p}}(\mathbf{a}) \cdot(\mathbf{l} \times(\mathbf{a} \times \mathbf{s}))\right]+
$$




$$
\left[\mathbf{a} \cdot\left(\left[\frac{\partial}{\partial x^{p}}(\mathbf{l}) \times(\mathbf{a} \times \mathbf{s})\right]+\left[\mathbf{l} \times \frac{\partial}{\partial x^{p}}(\mathbf{a} \times \mathbf{s})\right]\right)\right] .
$$

Removing the first expanded cross product term because $\mathbf{l}$ isn't dependent on $x^{p}$, and expanding the second cross product term using the same property previously employed results in

$$
\begin{gathered}
\frac{\partial P A}{\partial x^{p}}=\left[\frac{\partial}{\partial x^{p}}(\mathbf{a}) \cdot(\mathbf{l} \times(\mathbf{a} \times \mathbf{s}))\right]+ \\
{\left[\mathbf{a} \cdot\left(\mathbf{l} \times\left[\frac{\partial}{\partial x^{p}}(\mathbf{a}) \times \mathbf{s}+\mathbf{a} \times \frac{\partial}{\partial x^{p}}(\mathbf{s})\right]\right)\right] .}
\end{gathered}
$$

As previously shown, $\mathbf{s}$ isn't dependent on $x^{p}$, so the final equation can be written as

$$
\frac{\partial P A}{\partial x^{p}}=\left[\frac{\partial}{\partial x^{p}}(\mathbf{a}) \cdot(\mathbf{l} \times(\mathbf{a} \times \mathbf{s}))\right]+\left[\mathbf{a} \cdot\left(\mathbf{l} \times\left[\frac{\partial}{\partial x^{p}}(\mathbf{a}) \times \mathbf{s}\right]\right)\right] .
$$

Finally, the derivative of $P B$ will be examined, and the starting point is

$$
\frac{\partial P B}{\partial x^{p}}=\frac{\partial}{\partial x^{p}}(P A-A l S M) .
$$

The derivative can be distributed, and the product rule applied, resulting in

$$
\frac{\partial P B}{\partial x^{p}}=\left(\frac{\partial}{\partial x^{p}}(P A)-\left[\frac{\partial}{\partial x^{p}}(A l) S M+A l \frac{\partial}{\partial x^{p}}(S M)\right]\right) .
$$

Since $S M$ isn't a function of $x^{p}$, the final form can be written as

$$
\frac{\partial P B}{\partial x^{p}}=\left(\frac{\partial}{\partial x^{p}}(P A)-\left[\frac{\partial}{\partial x^{p}}(A l) S M\right]\right) .
$$

Using the definition of $A l=\mathbf{n} \cdot(\mathbf{s} \times \mathbf{a})$, where $\mathbf{a}$ is the only term in $A l$ that is dependent on $x^{p}$, results in the final equation for $\frac{\partial P B}{\partial x^{p}}$, which can be written as

$$
\frac{\partial P B}{\partial x^{p}}=\left(\frac{\partial}{\partial x^{p}}(P A)-\left[\mathbf{n} \cdot\left(\mathbf{s} \times \frac{\partial}{\partial x^{p}}(\mathbf{a})\right) S M\right]\right) .
$$


Lastly, the derivative of $D N O M$ must be calculated. The initial derivative can be written as

$$
\frac{\partial}{\partial x^{p}}(D N O M)=\frac{\partial}{\partial x^{p}}\left(P A P B+\left(P N^{2}\right) A B\left(S M^{2}\right)\right) .
$$

Distributing the derivatives over the sum and applying the product rule gives

$$
\frac{\partial}{\partial x^{p}}(D N O M)=\left[\frac{\partial}{\partial x^{p}}(P A) P B+P A \frac{\partial}{\partial x^{p}}(P B)\right]+\frac{\partial}{\partial x^{p}}\left(\left(P N^{2}\right) A B\left(S M^{2}\right)\right) .
$$

Applying the product rule to the second derivative term results in

$$
\begin{gathered}
\frac{\partial}{\partial x^{p}}(D N O M)=\left[\frac{\partial}{\partial x^{p}}(P A) P B+P A \frac{\partial}{\partial x^{p}}(P B)\right]+ \\
{\left[\left(\frac{\partial}{\partial x^{p}}\left(P N^{2}\right) A+P N^{2} \frac{\partial}{\partial x^{p}}(A)\right) B\left(S M^{2}\right)+\right.} \\
\left.\left(P N^{2}\right) A\left(\frac{\partial}{\partial x^{p}}(B)\left(S M^{2}\right)+B \frac{\partial}{\partial x^{p}}\left(S M^{2}\right)\right)\right] .
\end{gathered}
$$

This equation shows that to compute the derivative of $D N O M$ it is necessary to know the derivatives of $A, B, P A$, and $P B$, which have already been found, along with the derivatives of $P N^{2}$ and $S M^{2}$. The remaining two derivatives are now found.

$$
\frac{\partial S M^{2}}{\partial x^{p}}=\frac{\partial}{\partial x^{p}}((\mathbf{s} \cdot \mathbf{m})(\mathbf{s} \cdot \mathbf{m}))
$$

Using the chain rule this becomes

$$
\frac{\partial S M^{2}}{\partial x^{p}}=2(\mathbf{s} \cdot \mathbf{m}) \frac{\partial}{\partial x^{p}}(\mathbf{s} \cdot \mathbf{m}),
$$

which, using the properties of dot products, results in

$$
\frac{\partial S M^{2}}{\partial x^{p}}=2(\mathbf{s} \cdot \mathbf{m})\left[\left(\frac{\partial}{\partial x^{p}}(\mathbf{s}) \cdot \mathbf{m}\right)+\left(\mathbf{s} \cdot \frac{\partial}{\partial x^{p}}(\mathbf{m})\right)\right] .
$$


Since neither $\mathbf{s}$ nor $\mathbf{m}$ are dependent on survey point location, both derivatives become zero, and therefore the the entire derivative is zero.

$$
\frac{\partial S M^{2}}{\partial x^{p}}=0
$$

For $P N^{2}$ the derivative is

$$
\frac{\partial P N^{2}}{\partial x^{p}}=\frac{\partial}{\partial x^{p}}\left(\left(\mathbf{P}_{\mathbf{j k}} \cdot \mathbf{n}\right)\left(\mathbf{P}_{\mathbf{j k}} \cdot \mathbf{n}\right)\right) .
$$

Using the chain rule this becomes

$$
\frac{\partial P N^{2}}{\partial x^{p}}=2\left(\mathbf{P}_{\mathbf{j k}} \cdot \mathbf{n}\right) \frac{\partial}{\partial x^{p}}\left(\mathbf{P}_{\mathbf{j k}} \cdot \mathbf{n}\right) .
$$

Applying the properties of dot products, and recognizing that $\mathbf{n}$ isn't a function of $x^{p}$, results in the final form of this derivative as follows

$$
\begin{aligned}
& \frac{\partial P N^{2}}{\partial x^{p}}=2\left(\mathbf{P}_{\mathbf{j k}} \cdot \mathbf{n}\right) n_{x} \\
& \frac{\partial P N^{2}}{\partial y^{p}}=2\left(\mathbf{P}_{\mathbf{j k}} \cdot \mathbf{n}\right) n_{y}
\end{aligned}
$$

and

$$
\frac{\partial P N^{2}}{\partial z^{p}}=2\left(\mathbf{P}_{\mathbf{j k}} \cdot \mathbf{n}\right) n_{z}
$$

where $n_{x}, n_{y}$, and $n_{z}$ are the components of the $\mathbf{n}$ vector corresponding to that specific axis. 


\section{C.1.4 Constant Strength Doublet}

The velocity influence equation for a constant strength doublet panel with four sides can be written in vector form as

$$
\vec{u}_{\text {const,doublet }}=\sum_{i=1}^{4} \frac{(\mathbf{a} \times \mathbf{b})(A+B)}{A B(A B+(\mathbf{a} \cdot \mathbf{b}))},
$$

where $\mathbf{a}=\left(x^{p}-x_{i}\right) \hat{i}+\left(y^{p}-y_{i}\right) \hat{j}+\left(z^{p}\right) \hat{k}$ and $\mathbf{b}=\left(x^{p}-x_{i+1}\right) \hat{i}+\left(y^{p}-y_{i+1}\right) \hat{j}+\left(z^{p}\right) \hat{k}$, which are the distances between the survey point, $\vec{x}^{p}$, and the beginning or end point on panel side $i$, and $A$ and $B$ are the magnitudes of $\mathbf{a}$ and $\mathbf{b}$ respectively.

The most simple option to complete the derivatives of this vector equation with respect to each axis direction is to leave Equation C.111 in its symbolic form and take the overall derivative while accounting for the dependence of each term on the variable which the derivative is being taken with, then apply the single derivatives that remain. Since the derivative of a sum is the sum of the derivatives, the sum in Equation C.111 can be pulled outside the derivative, and simply applied to the derivative equation when calculating the stretching term. As such it will be left out of the following equations for simplicity. Because the derivatives with respect to $x^{p}$, $y^{p}$, and $z^{p}$ are identical, the derivative, $\frac{\partial \vec{u}_{\text {const,doublet }}}{\partial x^{p}}$, will be calculated. The first step is to use the product rule to break the single derivative into two derivatives, where

$$
\begin{aligned}
& u=(\mathbf{a} \times \mathbf{b})(A+B) \text { and } v=\frac{1}{A B(A B+(\mathbf{a} \cdot \mathbf{b}))}, \\
& \frac{\partial \vec{u}_{\text {const }, \text { doublet }}}{\partial x^{p}}=\frac{\frac{\partial}{\partial x^{p}}((\mathbf{a} \times \mathbf{b})(A+B))}{A B(A B+(\mathbf{a} \cdot \mathbf{b}))}+ \\
& (\mathbf{a} \times \mathbf{b})(A+B) \frac{\partial}{\partial x^{p}}\left(\frac{1}{A B(A B+(\mathbf{a} \cdot \mathbf{b}))}\right) .
\end{aligned}
$$


Because of the large number of terms in this equation it is easiest to break it into two terms and handle them independently, and then recombine them once all simplification has been completed. In this case that gives

$$
\operatorname{term} 1=\frac{\frac{\partial}{\partial x^{p}}((\mathbf{a} \times \mathbf{b})(A+B))}{A B(A B+(\mathbf{a} \cdot \mathbf{b}))}
$$

and

$$
\operatorname{term} 2=(\mathbf{a} \times \mathbf{b})(A+B) \frac{\partial}{\partial x^{p}}\left(\frac{1}{A B(A B+(\mathbf{a} \cdot \mathbf{b}))}\right) .
$$

Starting with term 1 the first step is to use the product rule, where $u=(\mathbf{a} \times \mathbf{b})$ and $v=(A+B)$, which results in

$$
\operatorname{term} 1=\frac{\frac{\partial}{\partial x^{p}}(\mathbf{a} \times \mathbf{b})(A+B)+(\mathbf{a} \times \mathbf{b}) \frac{\partial}{\partial x^{p}}(A+B)}{A B(A B+(\mathbf{a} \cdot \mathbf{b}))} .
$$

This can then be simplified even further by distributing the derivative into the cross product as well as into the sum. These two changes result in the final form of term 1 , which can be written as

$$
\operatorname{term} 1=\frac{\left[\left(\frac{\partial}{\partial x^{p}}(\mathbf{a}) \times \mathbf{b}\right)+\left(\mathbf{a} \times \frac{\partial}{\partial x^{p}}(\mathbf{b})\right)\right](A+B)+(\mathbf{a} \times \mathbf{b})\left(\frac{\partial}{\partial x^{p}}(A)+\frac{\partial}{\partial x^{p}}(B)\right)}{A B(A B+(\mathbf{a} \cdot \mathbf{b}))} .
$$

Next, the simplified form of term 2 can be found by first applying the quotient rule, which results in

$$
\operatorname{term} 2=(\mathbf{a} \times \mathbf{b})(A+B)\left(\frac{(0)-\frac{\partial}{\partial x^{p}}(A B(A B+(\mathbf{a} \cdot \mathbf{b})))}{[A B(A B+(\mathbf{a} \cdot \mathbf{b}))]^{2}}\right) .
$$

The product rule can then be applied here, where $u=((A)(B))$ and $v=(A B+(\mathbf{a} \cdot \mathbf{b}))$, 
which results in

$$
\begin{gathered}
\operatorname{term} 2=(\mathbf{a} \times \mathbf{b})(A+B)\left(\frac{-\frac{\partial}{\partial x^{p}}(A B)(A B+(\mathbf{a} \cdot \mathbf{b}))}{[A B(A B+(\mathbf{a} \cdot \mathbf{b}))]^{2}}-\right. \\
\left.\frac{(A B) \frac{\partial}{\partial x^{p}}((A B+(\mathbf{a} \cdot \mathbf{b})))}{[A B(A B+(\mathbf{a} \cdot \mathbf{b}))]^{2}}\right)
\end{gathered}
$$

From here the product rule is again applied, now with $u=A$ and $v=B$, resulting in

$$
\begin{gathered}
\operatorname{term} 2=(\mathbf{a} \times \mathbf{b})(A+B)\left(\frac{\left(-A \frac{\partial}{\partial x^{p}}(B)-B \frac{\partial}{\partial x^{p}}(A)\right)(A B+(\mathbf{a} \cdot \mathbf{b}))}{[A B(A B+(\mathbf{a} \cdot \mathbf{b}))]^{2}}-\right. \\
\left.\frac{(A B) \frac{\partial}{\partial x^{p}}((A B+(\mathbf{a} \cdot \mathbf{b})))}{[A B(A B+(\mathbf{a} \cdot \mathbf{b}))]^{2}}\right)
\end{gathered}
$$

Next, the derivative of a sum is the sum of the derivatives, so

$$
\begin{gathered}
\operatorname{term} 2=(\mathbf{a} \times \mathbf{b})(A+B)\left(\frac{\left(-A \frac{\partial}{\partial x^{p}}(B)-B \frac{\partial}{\partial x^{p}}(A)\right)(A B+(\mathbf{a} \cdot \mathbf{b}))}{[A B(A B+(\mathbf{a} \cdot \mathbf{b}))]^{2}}-\right. \\
\left.\frac{(A B)\left[\frac{\partial}{\partial x^{p}}(A B)+\frac{\partial}{\partial x^{p}}(\mathbf{a} \cdot \mathbf{b})\right]}{[A B(A B+(\mathbf{a} \cdot \mathbf{b}))]^{2}}\right)
\end{gathered}
$$

Finally, applying the product rule once again with $u=A$ and $v=B$, as well as applying the definition of the derivative of a dot product results in the final formulation of term 2 as follows

$$
\begin{aligned}
& \operatorname{term} 2=(\mathbf{a} \times \mathbf{b})(A+B)\left(\frac{\left[-A \frac{\partial}{\partial x^{p}}(B)-B \frac{\partial}{\partial x^{p}}(A)\right](A B+(\mathbf{a} \cdot \mathbf{b}))}{[A B(A B+(\mathbf{a} \cdot \mathbf{b}))]^{2}}-\right. \\
& \left.\frac{(A B)\left[\left(A \frac{\partial}{\partial x^{p}}(B)+B \frac{\partial}{\partial x^{p}}(A)\right)+\left(\frac{\partial}{\partial x^{p}}(\mathbf{a}) \cdot \mathbf{b}+\mathbf{a} \cdot \frac{\partial}{\partial x^{p}}(\mathbf{b})\right)\right]}{[A B(A B+(\mathbf{a} \cdot \mathbf{b}))]^{2}}\right)
\end{aligned}
$$

Now term 1 and term 2 are both written in forms where the only derivatives that remain to be solved for are those of $a, b, A$, and $B$. The derivatives with respect to $A$ and $B$ are easily found, and written

$$
\frac{\partial A}{\partial x^{p}}=\frac{x^{p}-x_{i}}{\sqrt{\left(x^{p}-x_{i}\right)^{2}+\left(y^{p}-y_{i}\right)^{2}+\left(z^{p}\right)^{2}}},
$$




$$
\begin{gathered}
\frac{\partial A}{\partial y^{p}}=\frac{y^{p}-y_{i}}{\sqrt{\left(x^{p}-x_{i}\right)^{2}+\left(y^{p}-y_{i}\right)^{2}+\left(z^{p}\right)^{2}}}, \\
\frac{\partial A}{\partial z^{p}}=\frac{z^{p}}{\sqrt{\left(x^{p}-x_{i}\right)^{2}+\left(y^{p}-y_{i}\right)^{2}+\left(z^{p}\right)^{2}}}, \\
\frac{\partial B}{\partial x^{p}}=\frac{x^{p}-x_{i+1}}{\sqrt{\left(x^{p}-x_{i+1}\right)^{2}+\left(y^{p}-y_{i+1}\right)^{2}+\left(z^{p}\right)^{2}}} \\
\frac{\partial B}{\partial y^{p}}=\frac{y^{p}-y_{i+1}}{\sqrt{\left(x^{p}-x_{i+1}\right)^{2}+\left(y^{p}-y_{i+1}\right)^{2}+\left(z^{p}\right)^{2}}}
\end{gathered}
$$

and

$$
\frac{\partial B}{\partial z^{p}}=\frac{z^{p}}{\sqrt{\left(x^{p}-x_{i+1}\right)^{2}+\left(y^{p}-y_{i+1}\right)^{2}+\left(z^{p}\right)^{2}}} .
$$

The derivatives with respect to $a$ and $b$ are also easily found, and can be written

$$
\begin{aligned}
\frac{\partial a}{\partial x^{p}} & =1 \hat{i}+0 \hat{j}+0 \hat{k} \\
\frac{\partial a}{\partial x^{p}} & =0 \hat{i}+1 \hat{j}+0 \hat{k} \\
\frac{\partial a}{\partial x^{p}} & =0 \hat{i}+0 \hat{j}+1 \hat{k} \\
\frac{\partial b}{\partial x^{p}} & =1 \hat{i}+0 \hat{j}+0 \hat{k} \\
\frac{\partial b}{\partial x^{p}} & =0 \hat{i}+1 \hat{j}+0 \hat{k}
\end{aligned}
$$

and

$$
\frac{\partial b}{\partial x^{p}}=0 \hat{i}+0 \hat{j}+1 \hat{k}
$$

The combination of the simple derivatives of $a, b, A$, and $B$, along with the symbolic derivatives of term 1 and term 2 provides the necessary derivative information for calculation of stretching due to a panel with a constant strength doublet distribution. 


\section{Appendix D}

\section{Panel Influence Verification}

Each off-body influencing relationship was verified through several methods because the off-body velocity and velocity gradient scans are so critical to the correctness of the proposed method. Functions for calculating off-body values for potential, velocity, and velocity gradient were constructed for point and constant strength source and doublet panels. The analytical results were compared with other formulations of identical influence or finite difference derivative approximations, through the relationship

$$
\nabla \mathbf{u}=\frac{\partial \mathbf{u}}{\partial \mathbf{x}}=\frac{\partial^{2} \Phi}{\partial \mathbf{x}^{2}}
$$

where $\mathbf{u}$ is the vector velocity at the point of interest and $\mathbf{x}$ is the vector location of the point of interest. In addition to the panel elements, the vortex particle scheme previously created was verified using the same process, though only comparing velocity and velocity gradient.

Equation D.1 tells us that a way to verify that there aren't any simple errors in the implementation of the off-body influence functions is to compare each analytical 
function with its equivalent numerical derivative. Since there is no term more basic than the potential, two separate formulations of the potential were constructed, and the results compared. Once those values matched, the finite difference derivative of the potential was then compared with analytical velocity influence functions. Additionally, the analytical velocity gradient was compared with finite difference first derivatives of velocity, and also finite difference second derivatives of potential. All of these separate measurements matching gave good evidence that the formulations were correctly implemented.

The study had several key features that enabled it to verify the correctness of the implementation of off-body influence functions under all applicable conditions. A single panel was used in each study, but several adjustments were made to the panel to ensure total correctness of all functions. The initial panel was flat, lying completely in the $x-y$ plane, with a collocation point at $(0,0,0)$. This meant that the local panel coordinate frame was identical to the global coordinate frame, thereby removing the necessity for coordinate transformations and ensuring that the influencing functions were free of bugs.

After the level, centered panel was confirmed to be accurate, the panel was placed at various angles relative to the global coordinate system, and then all functions were reverified. This alteration in the panel meant that now a transformation would be required for certain functions to complete influence calculations correctly. This ensured that any function that required a coordinate transformation to local panel coordi- 
nates had that transformation implemented correctly. With all the transformations implemented, the panel was returned briefly to level to ensure that the addition of the transformation didn't change anything about the initial results.

Once it was clear that all the functions had the panel transformation implemented correctly, the panel collocation point was shifted away from $(0,0,0)$. This check would ensure that any errors regarding nonzero panel collocation points was corrected, as no realistic panel is likely to be centered at exactly $(0,0,0)$.

With accurate quadrilateral panel influence verified for panels that are either flat or angled, and that aren't necessarily centered at the global origin, the next step was to verify that influence from triangular panels could be accurately calculated. For the point element equations, the triangular panel is not a concern as long as the area can still be correctly calculated. To find the influence from a constant strength panel it was necessary to break down each influencing equation into the influence caused by each side of a panel. A triangular panel was indicated in the code by a repeated panel corner point. To correctly account for a triangular panel it was necessary to ensure that no singularities were induced in the code in the case that a given panel side had a length of zero.

For each of these cases the influences were compared at nine different points. A survey point was created in each of the eight octants, so that each possible sign combination would be examined for a point of influence. Additionally, a ninth survey point was created to place a point in the plane of the panel, to examine if there were 
any singularity effects, and ensure proper treatment of in-plane influences. A sample of the points distributions relative to the panel is shown in Figure D.1.

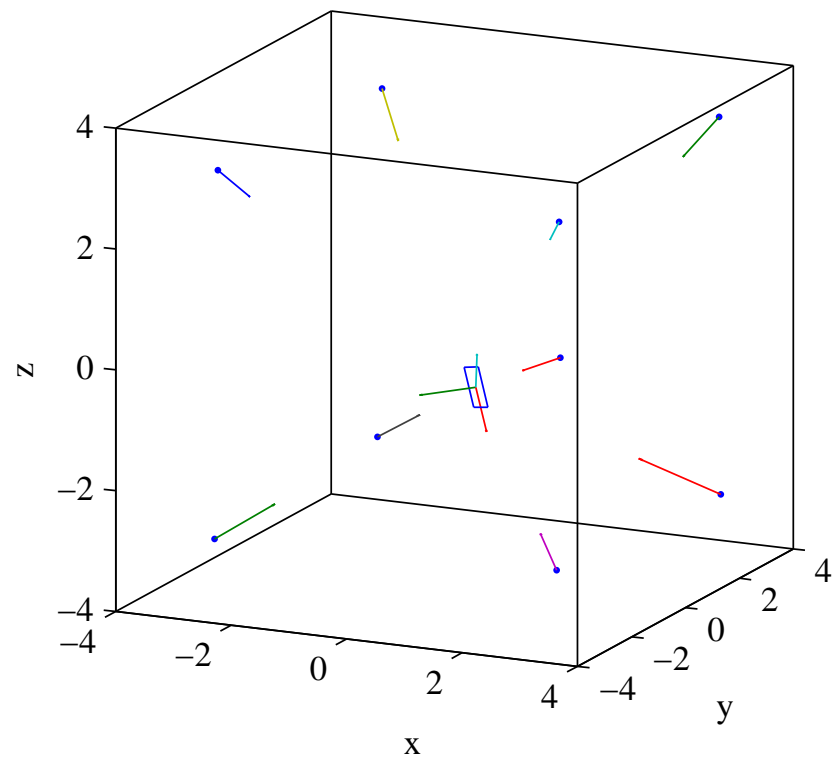

Figure D.1: Survey with corresponding velocity vectors relative to arbitrarily oriented panel with a constant strength source element.

\section{D.1 Panel Potential}

The potential from each element and distribution type was verified through independent calculations. Two separate methods were used to calculate potential at each survey point of interest, a formulation from Katz and Plotkin ${ }^{66}$ and a formulation used in the panel code VSAERO ${ }^{68}$ Browne and Ashby ${ }^{67}$ provide a side by side comparison of the nomenclature used in VSAERO with that used in the potential flow work of Hess. ${ }^{61}$ The resulting potential value from each of these formulations is compared, with differences being on the order of magnitude of $1 e^{-17}$, which is approximately the smallest possible value representable in double precision. 


\section{D.1.1 Point Doublet}

The point equations are used to represent a constant strength panel influence when the survey point is far from the panel. As such, the area is taken into account to ensure that the point element has the same strength that the entire constant strength panel it represents had.

The potential influence due to a point doublet element is written by Katz and Plotkin as

$$
\Phi_{d}=\frac{-\mu A}{4 \pi} \frac{z}{\left(\left(x-x_{0}\right)^{2}+\left(y-y_{0}\right)^{2}+(z)^{2}\right)^{3 / 2}},
$$

where $\mu$ is the point doublet strength, $A$ is the panel area, $(x, y, z)$ is the field point at which the potential value is desired, and $\left(x_{0}, y_{0}, z_{0}\right)$ is the location of the point source, where both are in panel coordinates, meaning that $z_{0}$ should be zero on the panel ${ }^{66}$

The values calculated in the field using Equation D.6 can be compared to an equation from VSAERO that is formulated slightly differently to obtain the same value. $\frac{68}{6}$ That equation can be written as

$$
\Phi_{d}=\frac{\sigma A P N}{4 \pi P_{j k}^{3}},
$$

where $P_{j k}$ is the magnitude of the vector distance between the survey point and the panel collocation point,

$$
P_{j k}=\mathbf{R}_{c, j}-\mathbf{R}_{c, k},
$$


and $P N$ is the dot product of $\mathbf{P}_{j k}$ and the panel normal vector,

$$
P N=\mathbf{P}_{j k} \cdot \mathbf{n}
$$

thereby providing definition of the orientation of the doublet in the same way that the $z$ term in the numerator of Equation D.2 does. The terms in Equation D.4 and D.5 can be seen graphically in Figure D.2.

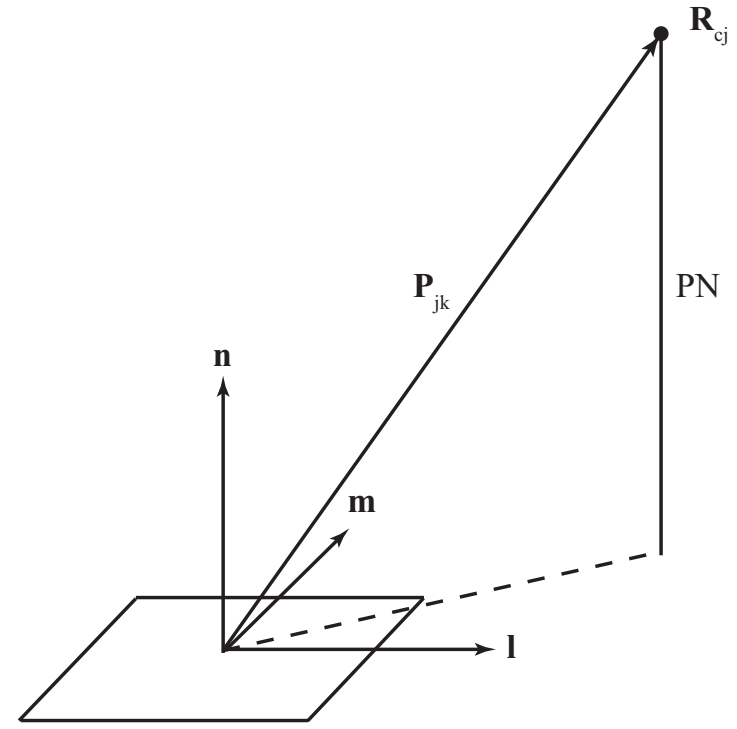

Figure D.2: Panel coordinate vectors and point of interest vector.

\section{D.1.2 Point Source}

Just like the point doublet, the point source equations are used to represent a constant strength source panel when the survey point is far from the panel. The potential caused by a point source element is written by Katz and Plotkin as

$$
\Phi_{s}=\frac{-\sigma A}{4 \pi \sqrt{\left(x-x_{0}\right)^{2}+\left(y-y_{0}\right)^{2}+(z)^{2}}},
$$


where $\sigma$ is the source strength, and the rest of the nomenclature remains the same as in Equation D.2. ${ }^{66}$

An identical potential value can again be found using a vector formulation of the potential, as defined in VSAERO ${ }^{68}$ That definition is

$$
\Phi_{s}=\frac{\sigma A}{4 \pi P_{j k}},
$$

Equation D.7 is identical to Equation D.6 except that it is written in vector form.

\section{D.1.3 Constant Strength Doublet}

The constant strength doublet potential formulation can be written in a similar manner to the point element equation, though the difference between the scalar and vector formulations is much more staggering in this case. The scalar equation is reproduced from Katz and Plotkin as $\sqrt{66}$

$$
\begin{aligned}
\Phi_{d}= & |z| \frac{\mu}{4 \pi}\left[\tan ^{-1}\left(\frac{m_{12} e_{1}-h_{1}}{z r_{1}}\right)-\tan ^{-1}\left(\frac{m_{12} e_{2}-h_{2}}{z r_{2}}\right)\right. \\
& +\tan ^{-1}\left(\frac{m_{23} e_{2}-h_{2}}{z r_{2}}\right)-\tan ^{-1}\left(\frac{m_{23} e_{3}-h_{3}}{z r_{3}}\right) \\
& +\tan ^{-1}\left(\frac{m_{34} e_{3}-h_{3}}{z r_{3}}\right)-\tan ^{-1}\left(\frac{m_{34} e_{4}-h_{4}}{z r_{4}}\right) \\
& \left.\left.+\tan ^{-1}\left(\frac{m_{41} e_{4}-h_{4}}{z r_{4}}\right)-\tan ^{-1}\left(\frac{m_{41} e_{1}-h_{1}}{z r_{1}}\right)\right]\right],
\end{aligned}
$$

where the terms are defined as

$$
\begin{gathered}
m_{k k+1}=\frac{y_{k+1}-y_{k}}{x_{k+1}-x_{k}}, \\
e_{k}=\left(x-x_{k}\right)^{2}+(z)^{2},
\end{gathered}
$$




$$
h_{k}=\left(x-x_{k}\right)\left(y-y_{k}\right), \quad k=1,2,3,4
$$

and $\left(x_{k}, y_{k}, z_{k}\right)$ is the $k^{t h}$ panel corner point.

The vector formulation of the doublet potential influence arrives at the same result. It is written in VSAERO as

$$
\Phi_{d}=\sum_{i=1}^{4} \Phi_{d, i}
$$

where the potential contribution is examined from each panel side due to a constant strength doublet distribution. The contribution from an individual panel side is

$$
\Phi_{d, i}=\tan ^{-1}\left(\frac{R N U M}{D N O M}\right) .
$$

The terms contributing to panel side potential can be broken down further as

$$
R N U M=S M P N(B P A-A P B)
$$

and

$$
D N O M=P A P B+P N^{2} A B S M^{2} .
$$

$P N$ is still defined as in Equation D.5. The rest of the terms can be further broken down as follows,

$$
\begin{gathered}
A=|\mathbf{a}|, \\
B=|\mathbf{b}|, \\
P A=P N^{2} S L+A l A M, \\
P B=P N^{2} S L+A l B M,
\end{gathered}
$$




$$
\begin{gathered}
S M=\mathbf{s} \cdot \mathbf{m}, \\
S L=\mathbf{s} \cdot \mathbf{l}, \\
A M=\mathbf{a} \cdot \mathbf{m}, \\
B M=\mathbf{b} \cdot \mathbf{m}, \\
A l=A M S L-A L S M,
\end{gathered}
$$

where $\mathbf{a}$ is the distance between the panel side initial corner point and the point of interest, $\mathbf{b}$ is the distance between the panel side end corner point and the point of interest, and $\mathbf{s}$ is the vector defining the panel side. As stated earlier, the formulations have drastically different appearances, but both calculate the same term, the potential contribution of a constant strength doublet panel, indicating that if the results of each formulation are identical then it is safe to say that both implementations are correct. Some of the major terms above are shown graphically in Figure D.3.

\section{D.1.4 Constant Strength Source}

The constant strength source potential can be found through different formulations, just as the two point influence potentials were. In the case of scalar components, the potential can be written as Katz and Plotkin did, 66

$$
\begin{aligned}
\Phi_{s}= & \frac{-\sigma}{4 \pi}\left[\left[\frac{\left(x-x_{1}\right)\left(y_{2}-y_{1}\right)-\left(y-y_{1}\right)\left(x_{2}-x_{1}\right)}{d_{12}} \log \left(\frac{r_{1}+r_{2}+d_{12}}{r_{1}+r_{2}-d_{12}}\right)\right.\right. \\
& +\frac{\left(x-x_{2}\right)\left(y_{3}-y_{2}\right)-\left(y-y_{2}\right)\left(x_{3}-x_{2}\right)}{d_{23}} \log \left(\frac{r_{2}+r_{3}+d_{23}}{r_{2}+r_{3}-d_{23}}\right)
\end{aligned}
$$




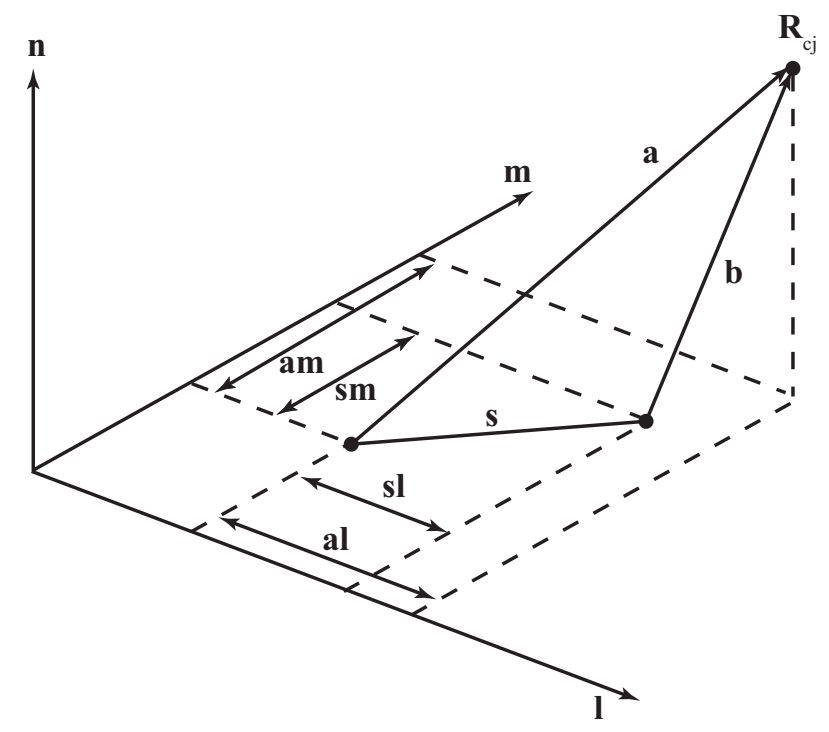

Figure D.3: Vectors corresponding to current panel side.

$$
\begin{gathered}
+\frac{\left(x-x_{3}\right)\left(y_{4}-y_{3}\right)-\left(y-y_{3}\right)\left(x_{4}-x_{3}\right)}{d_{34}} \log \left(\frac{r_{3}+r_{4}+d_{34}}{r_{3}+r_{4}-d_{34}}\right) \\
\left.+\frac{\left(x-x_{4}\right)\left(y_{1}-y_{4}\right)-\left(y-y_{4}\right)\left(x_{1}-x_{4}\right)}{d_{41}} \log \left(\frac{r_{4}+r_{1}+d_{41}}{r_{4}+r_{1}-d_{41}}\right)\right] \\
-|z|\left[\tan ^{-1}\left(\frac{m_{12} e_{1}-h_{1}}{z r_{1}}\right)-\tan ^{-1}\left(\frac{m_{12} e_{2}-h_{2}}{z r_{2}}\right)\right. \\
+\tan ^{-1}\left(\frac{m_{23} e_{2}-h_{2}}{z r_{2}}\right)-\tan ^{-1}\left(\frac{m_{23} e_{3}-h_{3}}{z r_{3}}\right) \\
+\tan ^{-1}\left(\frac{m_{34} e_{3}-h_{3}}{z r_{3}}\right)-\tan ^{-1}\left(\frac{m_{34} e_{4}-h_{4}}{z r_{4}}\right) \\
\left.\left.+\tan ^{-1}\left(\frac{m_{41} e_{4}-h_{4}}{z r_{4}}\right)-\tan ^{-1}\left(\frac{m_{41} e_{1}-h_{1}}{z r_{1}}\right)\right]\right]
\end{gathered}
$$

where the new terms are defined as

$$
d_{k k+1}=\operatorname{sqrt}\left(x_{k+1}-x_{k}\right)^{2}+\left(y_{k+1}-y_{k}\right)^{2},
$$

and

$$
r_{k}=\operatorname{sqrt}\left(x-x_{k}\right)^{2}+\left(y-y_{k}\right)^{2}+(z)^{2} .
$$


The same value for potential from a constant strength source panel can be found through vector mathematics, using the following relationships from VSAERO $\frac{68}{67}$ The potential is equal to the potential contribution from each of the panels sides as follows,

$$
\Phi_{s}=\sum_{i=1}^{4} \Phi_{s, i}
$$

where if the panel is a triangle then the contribution from the nonexistent panel side is simply $\Phi_{s, i}=0$. The contribution from an individual panel side can be found as

$$
\Phi_{i}=A l G L-P N \Phi_{d, i}
$$

where the same components are used as appeared in the vector form of the constant strength doublet equation. The additional term in this equation can be defined as

$$
G L=\frac{1}{S} \log \left(\frac{A+B+S}{A+B-S}\right),
$$

where

$$
S=|\mathbf{b}-\mathbf{a}| .
$$

\section{D.2 Panel Velocity}

Once the potential influences were verified, the next step was to verify the velocity influence in the field. This was done using the relationship in Equation D.1, which states that the velocity at a point in the field is equal to the derivatives of the potential in each axis component. Thus, the analytical velocity could be calculated at a point in the field, and the potential could be found and differentiated using a finite difference. 
The calculation of velocity through differentiating potential was conducted by surveying the potential at a desired step size away from the point of interest in each axis individually. The step size corresponded to the delta term used in the second order central difference. The central difference equation can be written as

$$
\frac{\partial f}{\partial x} \approx \frac{f(x+\Delta)-f(x-\Delta)}{2 \Delta}
$$

where $\Delta$ is the step size, and $f$ is the function to be differentiated. Each axis component of the velocity was found using a separate finite difference calculation, where the $\Delta$ step was applied only in the axis whose velocity component was the desired result. Alternatively, this can be stated as

$$
\begin{aligned}
& u=\frac{\partial \Phi}{\partial x} \approx \frac{\Phi(\mathbf{x}+[\Delta, 0,0])-\Phi(\mathbf{x}-[\Delta, 0,0])}{2 \Delta} \\
& v=\frac{\partial \Phi}{\partial y} \approx \frac{\Phi(\mathbf{x}+[0, \Delta, 0])-\Phi(\mathbf{x}-[0, \Delta, 0])}{2 \Delta} \\
& w=\frac{\partial \Phi}{\partial z} \approx \frac{\Phi(\mathbf{x}+[0,0, \Delta])-\Phi(\mathbf{x}-[0,0, \Delta])}{2 \Delta} .
\end{aligned}
$$

The potential functions chosen to be used in the finite differences were the implementation of the Katz and Plotkin code from Equations D.2, D.6, D.8, and D.25, although the choice was arbitrary because the VSAERO potential functions had already been proven to yield identical results.

It is important to note that the derivative is being taken with respect to the point of interest. This means that the point of interest is the value moved in the finite differences, and so the potential is evaluated at several different points in the field. 
Throughout the finite difference, the panel remains stationary, staying at the same orientation, and without moving the panel corner points.

\section{D.2.1 Point Source}

The velocity functions for the point source element were written from the Katz and Plotkin formulations. The resulting equations are

$$
\begin{aligned}
u_{p t, \text { source }} & =\frac{\sigma A\left(x-x_{0}\right)}{4 \pi\left[\left(x-x_{0}\right)^{2}+\left(y-y_{0}\right)^{2}+\left(z-z_{0}\right)^{2}\right]^{3 / 2}}, \\
v_{p t, \text { source }} & =\frac{\sigma A\left(y-y_{0}\right)}{4 \pi\left[\left(x-x_{0}\right)^{2}+\left(y-y_{0}\right)^{2}+\left(z-z_{0}\right)^{2}\right]^{3 / 2}}, \\
w_{p t, \text { source }} & =\frac{\sigma A\left(z-z_{0}\right)}{4 \pi\left[\left(x-x_{0}\right)^{2}+\left(y-y_{0}\right)^{2}+\left(z-z_{0}\right)^{2}\right]^{3 / 2}} .
\end{aligned}
$$

Because the influence of a point source is only a function of radial distance from the element, there is no need to transform coordinate systems. The influence function becomes singular when the point of interest is at the location of the element.

\section{D.2.2 Point Doublet}

The velocity functions for the point doublet element were also written from Katz and Plotkin formulations, resulting in

$$
\begin{aligned}
& u_{p t, \text { doublet }}=\frac{3 \mu A}{4 \pi} \frac{\left(x-x_{0}\right)\left(z-z_{0}\right)}{4 \pi\left[\left(x-x_{0}\right)^{2}+\left(y-y_{0}\right)^{2}+\left(z-z_{0}\right)^{2}\right]^{5 / 2}}, \\
& v_{p t, \text { doublet }}=\frac{3 \mu A}{4 \pi} \frac{\left(y-y_{0}\right)\left(z-z_{0}\right)}{4 \pi\left[\left(x-x_{0}\right)^{2}+\left(y-y_{0}\right)^{2}+\left(z-z_{0}\right)^{2}\right]^{5 / 2}},
\end{aligned}
$$

and

$$
w_{p t, \text { doublet }}=\frac{-\mu A}{4 \pi} \frac{\left(x-x_{0}\right)^{2}+\left(y-y_{0}\right)^{2}-2\left(z-z_{0}\right)^{2}}{4 \pi\left[\left(x-x_{0}\right)^{2}+\left(y-y_{0}\right)^{2}+\left(z-z_{0}\right)^{2}\right]^{5 / 2}},
$$


where $\mu$ is the point doublet strength, $A$ is still the panel area, and $\left(x_{0}, y_{0}, z_{0}\right)$ and $(x, y, z)$ are still the element location and survey point respectively.

Again, the area of the panel is taken into account when computing influence from the point doublet to ensure that the strength is equal to that of a doublet panel with a constant strength distribution in the farfield. Unlike the point source, the doublet has an orientation, which depends on the normal vector to the panel that the doublet is representing. As such, the Katz and Plotkin velocity formulations require that all inputs are in the panel coordinate frame, and the resulting velocity is correspondingly in the panel coordinate frame as well. As with the point source, the doublet is singular when the point of interest is at the location of the element.

\section{D.2.3 Constant Strength Source}

As with the potential influence, the velocity influence of constant strength panels is much more complex to define. The formulation used in the current research was implemented in the panel code VSAERO and defined in the VSAERO theory document. ${ }^{68}$ It uses the same components that went into the constant strength source and doublet potential. The final equation for velocity is again a sum of the contributions from each panel side, written as

$$
\mathbf{V}_{s}=\sum_{i=1}^{4} \mathbf{V}_{s, i}
$$

where the contribution from side $i$ is written as

$$
\mathbf{V}_{s, i}=G L(S M \mathbf{l}-S L \mathbf{m})+\Phi_{d, i} \mathbf{n}
$$


The calculation is conducted entirely in one coordinate frame. If global position information is input then the resulting velocity will be in global coordinates. If the input information is in local coordinates, and the transformation matrix is changed to an identity matrix, to represent the transformation matrix in local coordinates, then the resulting velocity will also be in local coordinates.

There are several conditional statements that are placed into the function to ensure robustness. First, a check is made to see if the survey point is in the plane of the panel, which is written in the function as $P N<t o l$, where PN is defined in Equation D.5 and $t o l$ is some tolerance close to zero. This check works because if the projection of the vector between the collocation point and the point of interest onto the panel normal vector is zero then the two vectors are orthogonal, and the point of interest necessarily lies in the panel plane. In this case the potential, $\Phi_{d, i}$, in Equation D.43 is set equal to zero. Additionally, if the panel is a triangle rather than a quadrilateral, or mathematically, $S_{i}<t o l$, then the velocity influence from the nonexistent fourth panel side is set equal to zero.

\section{D.2.4 Constant Strength Doublet}

The constant strength doublet velocity influence is slightly more complex than that of the point doublet, but alteration can be made that does drastically reduce the complexity of the formulation. The alteration is possible because the influence of a constant strength panel is the same as the influence if each panel edge was turned into a vortex filament. This means that the total velocity is just the summation of 
the influence from each panel side. The equation again uses the nomenclature from the constant strength source and doublet potential equations, and can be written as

$$
\mathbf{V}_{d}=\sum_{i=1}^{4} \mathbf{V}_{d, i}
$$

where the contribution from side $i$ is

$$
\mathbf{V}_{i, d}=\frac{(\mathbf{a} \times \mathbf{b})(A+B)}{A B(A B+(\mathbf{a} \cdot \mathbf{b}))}
$$

In Equation D.45 all of the terms are dependent on the current panel side being examined.

This calculation can be done entirely in global coordinates, which removes the necessity to transform to and from panel coordinate systems for each calculation. The computation could be completed in panel coordinates, if for some reason that was desired, and then the resulting velocity would also be in panel coordinates.

The simplicity of the formulation doesn't require any conditional statements. Additionally, Equation D.45 is the result of a slight reordering. This alternate expression reduces the occurrence of singularities from the equation. In the traditional form, any point in line with one of the vortex filaments results in a singularity. The new formulation reduces the singularity to only occurring when the point is on the panel side, rather than simply in line with it. This is necessary, because with thousands of panels, all at different angles, it is difficult to avoid being in line with at least one panel edge, but there is generally no reason to survey velocity on a given panel edge. 


\section{D.3 Velocity Gradient}

The final term to be verified was the velocity gradient, which is traditionally not a valuable term in the same way that potential or velocity are. In the particle scheme however, the velocity gradient becomes crucial. This is because it appears in the vortex stretching term, which is critical to correct simulation results.

The velocity gradient was verified in two ways. The analytical velocity gradient results were compared to both the first derivative of the analytical velocity and the second derivative of the potential for each element type. Because explicit formulations for the velocity gradient were not readily available in literature, they were all derived for this research. The analytical functions were found from derivatives of analytical velocity equations. The derivations are extremely long due to the fact that for each element and distribution type there are nine terms corresponding to the vector derivative $\frac{\partial \mathbf{V}}{\partial \mathbf{x}}$. As with the derivatives for velocity, the derivatives of velocity gradient are taken by moving the point of interest. The influencing panel maintains its orientation and position throughout the study. The reader is directed to Appendix C to find the exact equations and their derivations.

Similar conditional statements and singularities appear in the velocity gradients as did in the velocity equations from which they were calculated. Additionally, the velocity gradient functions require coordinate transformations only in the instances that their corresponding velocity equations did. The velocity gradient is the most computationally expensive of the flowfield values to calculate because there is an 
increase in the number of terms to be found for any single point in the flow.

\section{D.4 Distribution Comparisons}

Once confidence was established in the velocity equations for the point and constant strength elements, the influence of the two distribution types is compared over various sweeps of the domain. This is important because it helps establish an understanding of when the point approximation is valid to replace a constant strength element. Ashby and Browne state an achieved 40 time reduction in computational cost when using the point source influence over the constant strength influence. 67 Thus, anytime the two distribution types have nearly identical velocity results it is ideal to use the point influence, and since the two influences converge to the same value as $r \rightarrow \infty$, there is a certain threshold where the error associated with the simplification becomes acceptable.

The basic setup was relatively simple, and was created to replicate comparisons reported by both Katz and Plotkin $\frac{66}{6}$ and a report about VSAERO and PMARC 67 There was no need to complicate this particular test, as all the complexities of the panel influences had been verified in the previous tests. For the distribution studies the influence from a single panel was analyzed. The panel was square, with panel side lengths equal to $c$, and was centered at $(0,0,0)$, meaning that local and global coordinate frames were the same.

Three different sweep types were conducted; one changing height above the collo- 
cation point, one traversing across the median of the panel, and one traversing across the diagonal of the panel. The traversing sweep types can be seen over the panel in Figure D.4. The surveys along the median and diagonal were each conducted at two different heights above the panel.

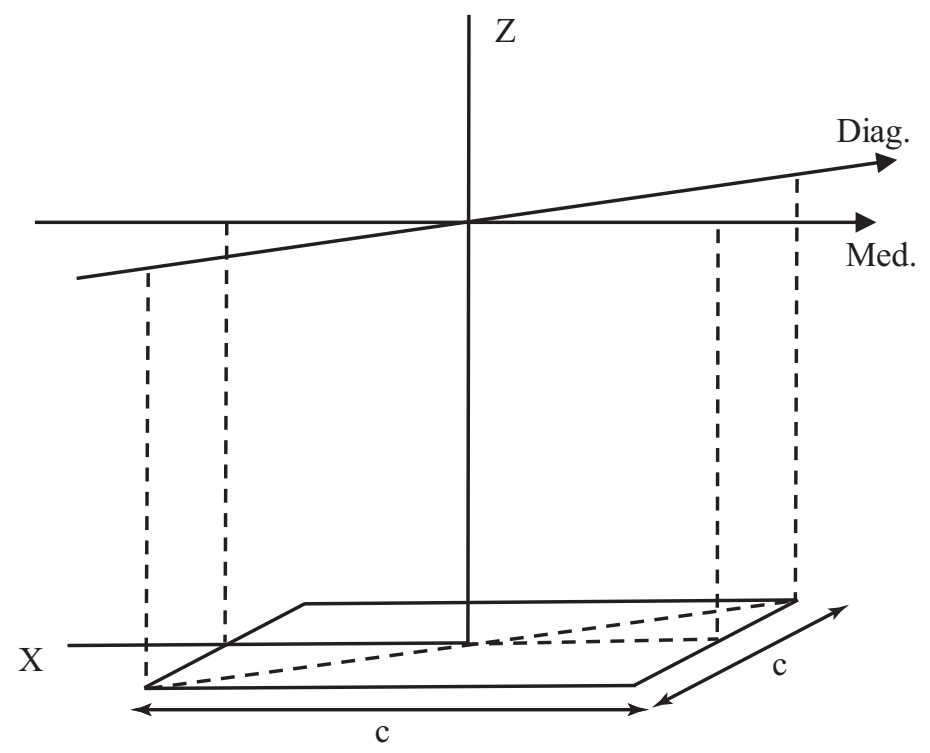

Figure D.4: Survey paths relative to the panel, with surveys taken in the $z$ axis as well as along the diagonal and the median of the panel.

The surveys were conducted individually for each element type. The axes on all the figures were set to match those on the resulting plots created by Katz and Plotkin for easy visual comparison. Examining Figures D.5, D.7, and D.8, it is clear that the largest difference between constant strength panel influence and the point approximation occurs when the point of interest is closest to the panel collocation 
point. Figure D.5, which agrees excellently with the plots it was modeled after,

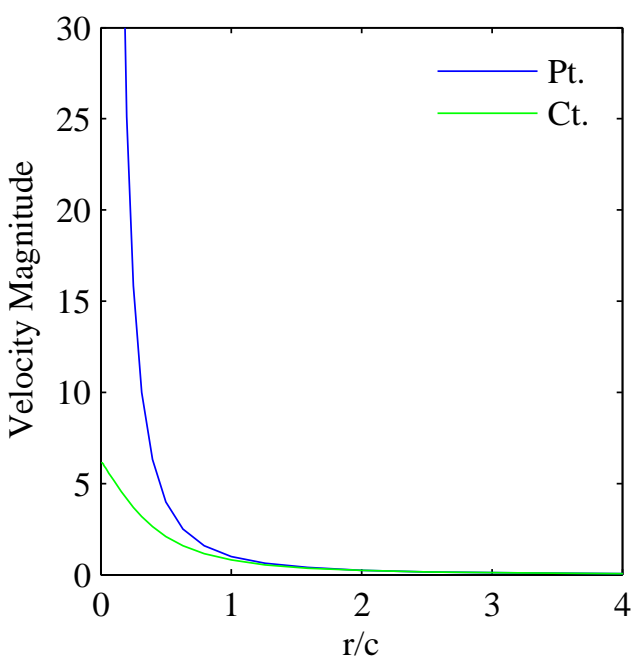

(a) Source

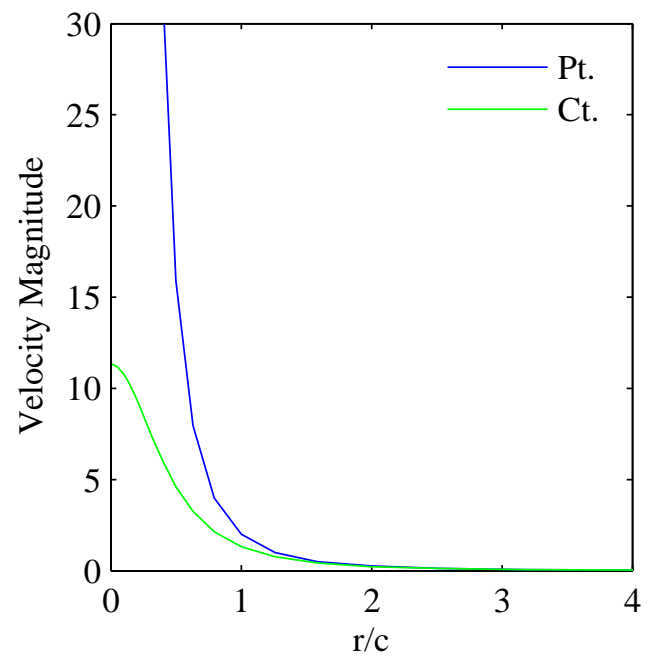

(b) Doublet

Figure D.5: Velocity influence of point (Pt.) and constant strength (Ct.) distribution types as distance above panel varies.

shows that the point source element has a definite singularity, with velocity tending toward infinity as the distance of the survey point from the element is reduced toward zero. This is in contrast to the constant strength element, which has a finite velocity influence at the collocation point of its own panel.

Figure D.5 can be examined in a slightly different way to see trends over a much greater sweep of points using a log plot. Figure D.6 shows just such a plot, which reveals two key features about the trends of the point versus the constant strength source. The important portions of the curve are the beginning and the end. Both show a linear trend on a log scale, indicating that there isn't any reason to believe the differences would level out if the span of the sweep was enlarged. It is apparent that the closer to the collocation point the survey point is, the higher the difference between 


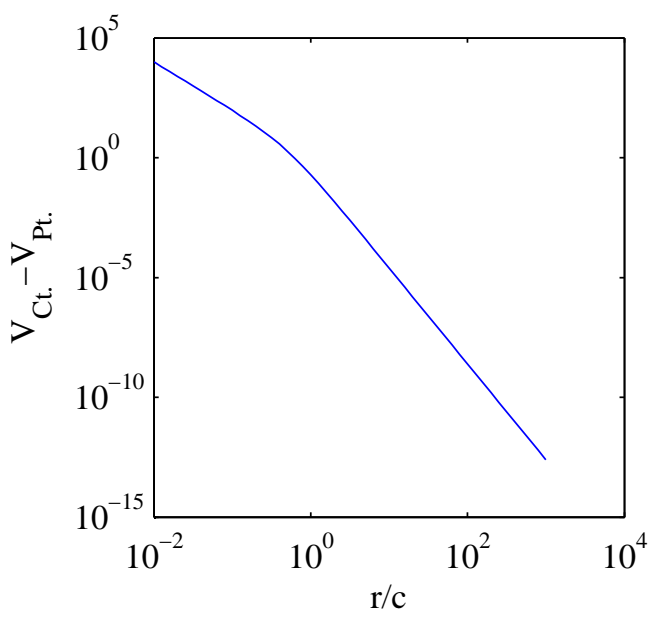

(a) Source

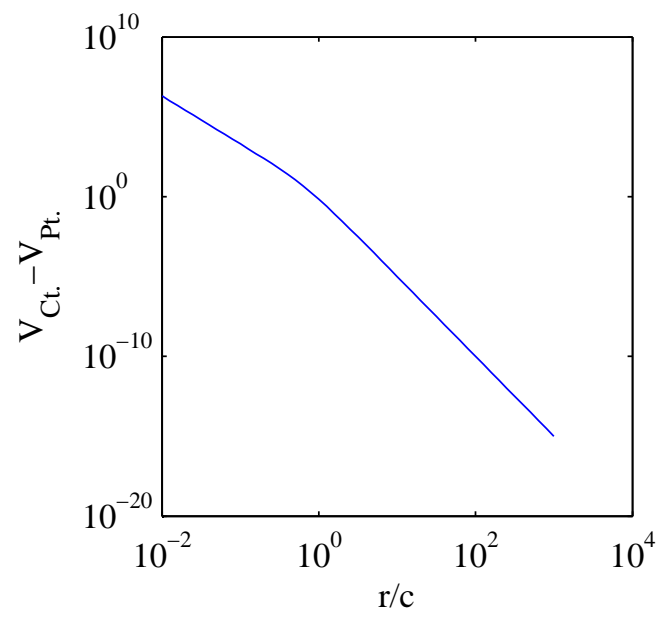

(b) Doublet

Figure D.6: Difference between constant strength and point approximation as height above panel varies.

the distributions is. This is because, as was seen in Figure D.5, the constant strength source has a finite influence at $r / c=0$, while the point source influence is infinite, and therefore the difference between the two becomes infinite. On the other end of the plot, a downward linear trend indicates that the difference will continue to zero as the distance away from the collocation panel tends toward infinity. Finally, Katz and Plotkin ${ }^{66}$ indicate that beyond three panel lengths away the point approximation is reasonable, while Browne and Ashby ${ }^{67}$ state that the assumption is valid beyond six panel lengths.

In addition to the survey varying the vertical distance away from the panel collocation point, a survey was conducted examining influence variation on a survey line over the panel diagonal. Figure D.7 displays the influence of a constant strength panel as compared to the point approximation for two different heights above the panel, 
$z / c=0.75$ and $z / c=3$. The agreement between Figure D.7 and the plots generated

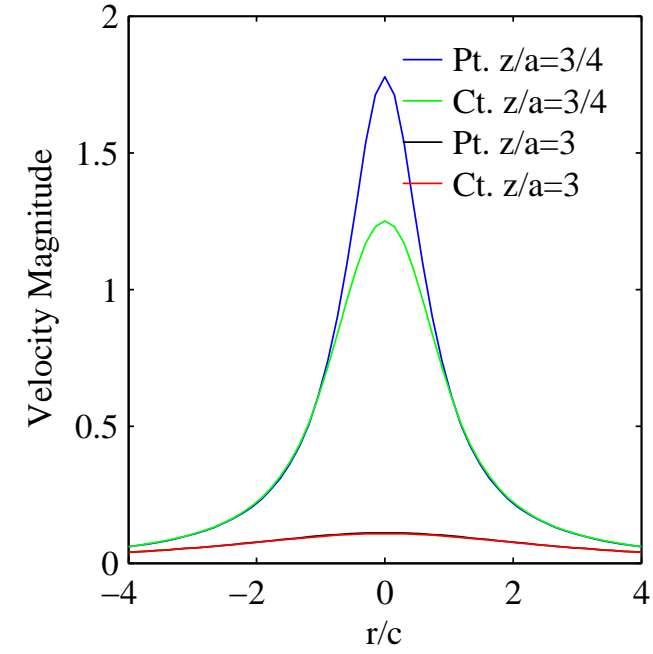

(a) Source

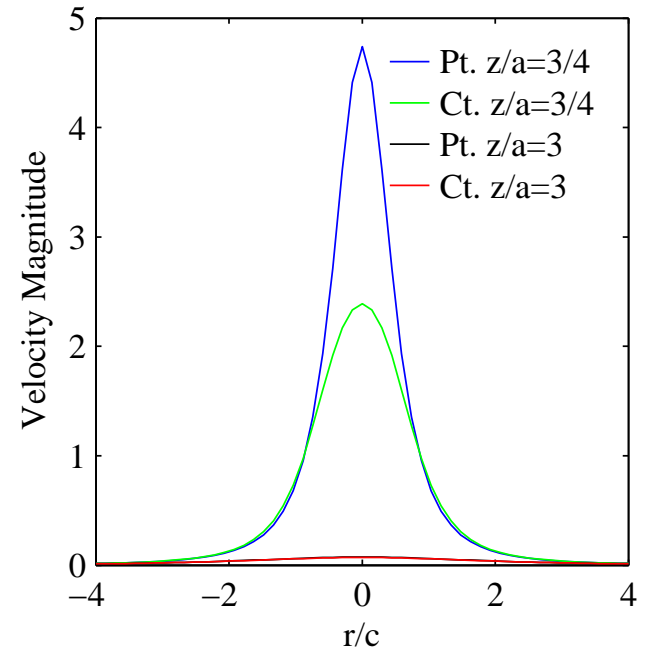

(b) Doublet

Figure D.7: Velocity influence of different distributions as distance along panel diagonal varies.

by Katz and Plotkin as well as Browne and Ashby are excellent, further indicating correct implementation of the influencing functions for both point and constant strength source and doublet panels. Although both the source and the doublet influences follow the same trend, with error between point and constant strength largest over the collocation point of the panel, the magnitude of the doublet influence for a unit strength is approximately double that of the source. Additionally, as would be indicated by the higher exponent in the denominator of the doublet influence Equations C.39, C.40, and C.41, the doublet influence tapers off toward zero at a much faster rate than the source panel does. This is apparent in both the smaller influence seen at $z / c=3$ above the panel, even though at $z / c=0.75$ the doublet influence was larger, as well as in the much steeper slope of the doublet influences at $z / c=0.75$. 
Finally, the survey over the panel median can be seen in Figure D.8. The shapes

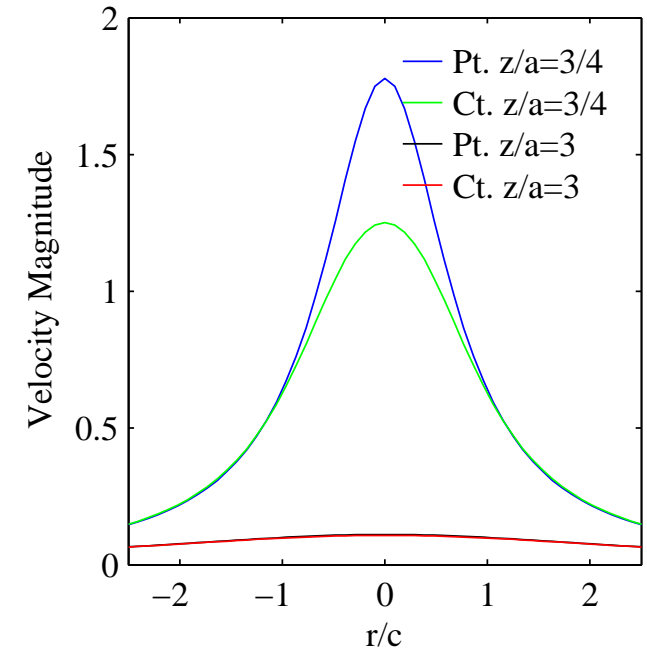

(a) Source

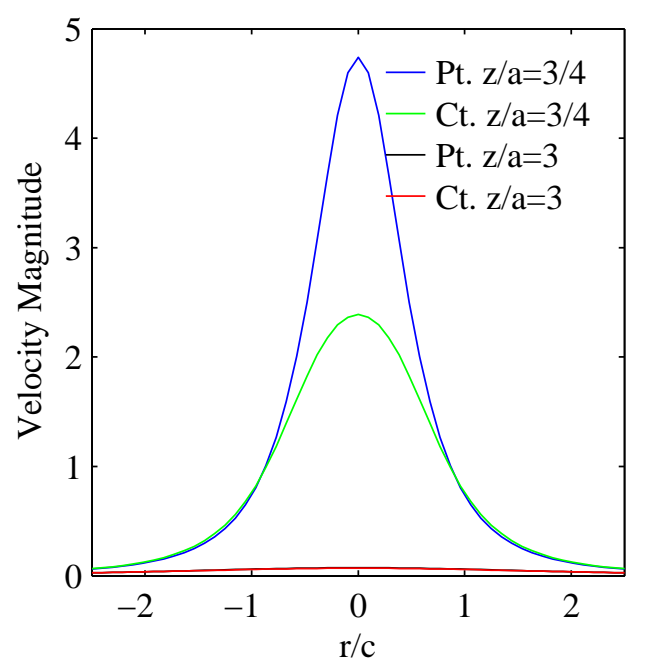

(b) Doublet

Figure D.8: Velocity influence of different distributions as distance along panel median varies.

and trends of Figure D.8 are identical to those found in the diagonal survey, with the magnitudes being slightly different due to the fact that the length being surveyed that falls over the panel is only $c$ instead of $\sqrt{2} c$ as it is in the diagonal survey. Again the agreement with the plots of previous research is excellent.

\section{D.5 Vortex Particle Stretching}

With the value of verifying the panel influence coefficients demonstrated, the same philosophy was applied to the vortex particle scheme. The goal with vortex particles was two fold, to provide another assurance that the particle velocity influence and stretching influence were free of bugs, and to ensure that the derivation describing the relationship between velocity and stretching, found in Appendix C was accurate. 
The scale of this verification was small in comparison to the panel influences. The only comparison being made in the vortex particle case was between the analytical stretching function, and the finite difference gradient of the velocity, applied in the derived relationship to achieve a stretching value.

The setup for this verification was also slightly different from the one used in the panel cases. First, the only allowable survey point for stretching due to vortex particles is at the location of a particle, meaning that there must be at least two particles to conduct the verification. This is because of the definition of stretching, which can be written as

$$
\frac{d}{d t} \alpha_{i}^{p}=\alpha_{l}^{p} \frac{\partial u_{l}}{\partial x_{i}}
$$

Equation D.46 shows that to obtain the stretching of particle $p$ it is necessary to know the vortex strength, as well as the velocity gradient at the location of particle $p$. The velocity gradient at particle $p$ is due to the influence from the other $q$ particles in the field, and the influence from those particles is dependent on the strengths at those $q$ locations. As such, the simplest way to conduct the verification is to survey the stretching at a single particle location due to other particles in the field.

In the case of this study, three particles were placed in the field, to provide asymmetric influence. Any number of particles could be included, with the computational cost increasing with each addition. Each particle was then assigned a random particle strength, again to insure asymmetry in the study that wouldn't be present if all the particles had identical strengths. To add an additional level of complexity, 
the analytical and numerical stretching were compared at each of the three particle locations.

Once a field of particles had been assembled, each with a unique strength and location, the velocity influence of each particle was found at each particle location. The finite difference derivatives were then taken using the same differencing scheme as the panel simulations, seen in Equation D.32. The numerical stretching was then calculated using the relationship from Equation D.46, so that the numerical stretching in each axis was known at each particle location.

After the numerical stretching was calculated the next task was to find the analytical stretching to compare with. This was again found at each particle due to the influence of all the particles in the field. The resulting matrix of partial derivatives, $\frac{\partial \alpha}{\partial \mathbf{x}}$, were compared. The differences between analytical and numerical stretching values for a finite difference step size of $10^{-6}$ were on the order of $10^{-7}$, where the stretching values being compared were on the order of $10^{-1}$.

The excellent matching between the numerical and analytical calculations of particle stretching indicate that the analytical velocity and stretching functions were implemented correctly, and that Equation D.46 correctly represents a method for calculating stretching, which the panel velocity gradients calculated earlier are applied to in order to obtain complete stretching influence at each particle location. 